\title{
RELAXATION TIME MEASUREMENTS IN ELECTRON PARAMAGNETIC RESONANCE
}

\author{
James J. Chang \\ (Ph. D. Thesis) \\ February 1971
}

AEC Contract No. W-7405-eng -48

\section{LAWRENCE RADIATION LABORATORY UNIVERSITY of CALIFORNIA BERKELEY}




\section{DISCLAIMER}

This report was prepared as an account of work sponsored by an agency of the United States Government. Neither the United States Government nor any agency Thereof, nor any of their employees, makes any warranty, express or implied, or assumes any legal liability or responsibility for the accuracy, completeness, or usefulness of any information, apparatus, product, or process disclosed, or represents that its use would not infringe privately owned rights. Reference herein to any specific commercial product, process, or service by trade name, trademark, manufacturer, or otherwise does not necessarily constitute or imply its endorsement, recommendation, or favoring by the United States Government or any agency thereof. The views and opinions of authors expressed herein do not necessarily state or reflect those of the United States Government or any agency thereof. 


\section{DISCLAIMER}

Portions of this document may be illegible in electronic image products. Images are produced from the best available original document. 


\section{PAGES $\mathrm{i}$ to $\mathrm{ii}$ WERE INTENTIONALLY LEFT BLANK}


RELAXATION TIME MEASUREMENTS IN FIECTRON PARAMAGN:TIC RESONANCE ights.

James J . Chang

Inorganic Materials Research Division, Lawrence Raliation Laboratory Department of Chemistry, University of California Berkeley, California 94720.

ABSTRACT

We have measured the electron paramagnetic resonance (EPI) spectra of $\mathrm{VO}\left(\mathrm{H}_{2} \mathrm{O}\right)_{5}^{2+}$, obtained from vanadyl perchlorate in solutions of both ordinary water and in heavy water, as a function of temperature. The linewidths in the vanadyl system are fit quite well by a combination of the relaxation theory for the tumbling of an anisotropic complex and the theory of spin-rotation interaction. The linewidths ror the vanadyl ion in ordinary water solution are well fit with the glass spectrum values of $g_{\|}=1.9311, g_{1}=1.9785, A_{\|}=-203.3$ gauss, $A_{1}=-75.8$ gauss, and the average solution values of $\mathrm{g}=1.9652, \mathrm{~A}=-115.9$ gauss. The correlation time js consistent with a hydrodynamic radius of $3.67 \mathrm{~A}$. In the deuterated system the radius is changed to $3.48 \mathrm{~A}$, with the other parameters remaining the sarne. The small residual linewidths which have been observed for the vanadyl ion in ordinary water are also observed in the deuterated system. These residual linewidths cannot be due to hyperfine interaction with ligand protons, and must be due to terms neglected in the relaxation theory.

We have also-measured the EPR spectra of $\mathrm{Cu}\left(\mathrm{H}_{2} \mathrm{O}\right)_{6}^{2+}$ obtained from copper perchlorate in aqueous solution as a function of temperature. Isotopically pure (99.62\%) copper -63 was used in ord 2 r to remove uncertainties which could occur in the normal isotropic mixture. The EPR. 
spectrum of the hexaquocopper (II) ion consists of a broad, unresolvel line. The spectra obtained as a function of temperature were digitizel with a data acquisition system. A least squares fit of Lorentz lineshapes to the digitized spectra was used to obtain the spectral parameters and linewidths. I'he Iinewidths are found to depend upon hyperfine component and to increase with increasing temperature. We have attempted to fit the linewidths to a combination of the spin-rotation interaction theory and the theory of a tumbling anisotropic species. It is not possible to fit the linewidths with both of these theories consistenly. Hence, we propose that a third relation mechanism contributes to the linewidths in the copper system.

We have developed a system for the measurement of spin-lattice relaxation times consisting of a puise-saturation spectrometer and a computer based data acquisition and analysis system. We have applied this system to the measurement of relaxation times for $\mathrm{Ni}^{2+}$ ions diluted in a host crystal of lanthanum magnesium nitrate. Relaxation times for the two strongest lines of the three line spectrum have been obtained. The data are vell fit by $\mathrm{T}_{1}^{-1}=.087 \mathrm{~T}^{2}\left(\mathrm{msec}^{-1}\right)$. This relationship is consistent with a phonon bottleneck process. Measurements of a more dilute crystal support this theory since the relaxation times are slightly shorter. 
TABLE OF CONT ENTS

\section{ABSTRACT}

I. INTRODUCTION-_- 1

II. THEORIES OF ELECTRON SPIN RELAXATIOI IN SOLUTIOR:-

A. Anisotropic g and A Tensors-_ 7

B. Spin-Rotation Interactions- 10

C. Al'tshuler and Valiev Mechanism 12

D. Inversion Mechanism-_- 15

E. Electric Field Fluctuation. Mechenisms-_- 16

III. VANADYL LINEWIDTHS-_ 20

A. Introduction- 20

B. Experịmental Methods-_a 23

c. Discussion and Results- 27

IV. COPPER IINEWIDTHS- 52

A. Introduction- 52

B. Experimerital Methods- 55

C. Discussion and Resultts- 56

V. SPIN-IATTICE RELAXATION TIME MEASUR: MENTS WITH AN ON-IINE COMPUTER-_- 90.

A. Introduction- 90

B. Experimental Methods- 93

C. Rale Eyuzliuns-_- 90

D. Discussion and Results-102 


$$
\text { -vi- }
$$

APPENDIX I. Lorentz Lineshapes and Least Squares Theory--_---- 113 APPENDIX II. Dịgital Programs- 119

A. Data Acquisition System Programs- 119

1. SUMTAP3- 119

2. FITESR-_- 134

3. GAUSS-_. 248

B. Simulation Programs-_-

1. IMITATE- 152

2. VAESR-_-_-_- 160

C. $T_{1}$ Analysis Program LOGLINE-167 REFERENCES- ACKNOWLEDGEMENTS- 


\section{INTRODUCTION}

Aithough magnetic resonance phenomena have been studied since 1946 , magnetic relaxation phenomena have been studied for a much longer time. The early non-resonant experiments (Gorter, 1.947) in magnetic phenomena served to establish many of the important ideas of magnetic relaxation. These experiments were usually interpreted in thermodynamic terms since many of the early experiments involved temperature measurements of magnetic systems. However, magnetic relaxation is usually more easily understood in terms of the resonance phenomenon.

According to the Bloch (1946) formalism a magnetic system may be characterized by two relaxation times which describe the return of a perturbed spir system to equilibrium. The spin-lattice relaxation time, $\mathrm{T}_{1}$, is the characteristic time for spin populations in the available energy levels to return to a Boltzmann equilibrium. In terms of the phenomenologicid Bloch equations this is known as the longitudinal relaxation time, or the time constant for the $z$ component of the magnetization. to return to $j$ ts equilibrium value. The spin-spin relaxation time, $\mathrm{T}_{2}$, is a measure of the time for the spins to come to equilibrium among them-. selves or to $d$ phase. $\mathrm{T}_{2}$ is also known as the transverse relaxation time since it is th? time constant for the $x$ and $y$ components of the magnetization to decay :o zero.

The spin-pin relaxation time, $\mathrm{T}_{2}$, is experimentally the easiest to determine and i.t is obtained from the widths of the neasured magnetic resonance line:. For a Lorentz line, $\mathrm{T}_{2}$ is related to the linewidth by

$$
\mathrm{T}_{2}^{-1}=\pi \sqrt{3} \Delta v
$$


where $\Delta v$ is the peak-to-peak separation in $\mathrm{Hz}$ of the first derivative .. of the absorption line. The independent variable in magnetic resonance experiments is usually magnetic field rather than frequenc $r$, and $\mathrm{T}_{2}$ is given by

$$
\mathrm{T}_{2}=\frac{h}{\mathrm{E}_{B}} \frac{1}{\pi \sqrt{3}} \frac{1}{\Delta \mathrm{H}}
$$

where $\Delta \mathrm{H}$ is the first derivative separation in gaus, $\mathrm{h}$ is Planck's constant, $B$, the Bohr magneton, and $g$, the " $g$ " value or spectroscopi a splitting constant for the absorption.

The spin lattice relaxation time, $\mathrm{T}_{1}$, is usually deternined by other methods such as c.w. saturation or pulse methods. However, the factors. which influence $T_{1}$ also influence $T_{2}$. In many cases, esperially in solution, $T_{1}=T_{2}$. In many cases when the value of $T_{1}$ is required, but is unknown, the equality is assumed. In any case, $\dot{\mathrm{T}}_{2}$ sets a lower limit for the value of $\mathrm{T}_{1}$.

The relaxation effects characterized by $\mathrm{T}_{1}$ and $\mathrm{T}_{2}$ can be related to time dependent magnetic or electric fields which influence the spins. There has been much interest in magnetic relaxation because of the information which nay be derived about the environment of the spins. The early theories of relaxation dealt mostly with the solid state, However, the liquid state has been of increasing interest since the original observation of magnetic resonance.

The original work on relaxation in solution was due to Bloembergen, Purcell and Pound (1948), hereinafter referred to as BPP. These workers computed relaxation times using tjme dependent perturbition theory and. correlation functions. They utilized the methods of the theory of Brownian 
motion and related the relaxation times to the bulk viscosity of the solution. This has attracted much interest in the possibility of studying the structure of liquids by means of magretic resonance linewidths in solution. Analysis of the linewidths could yield a correlation time which is characteristic of the solvent and of the complex being studied.

Indeed, nuclear magnetic resonance (NMR) linewidths have been extensively used to provide information about ion-solvent and ion-ion interactions (Burgess and Symons, 1968). The temperature dependence of ${ }^{17} 0 \mathrm{NMR}$ linewidths has been extensively used to study waters of hydration and their rates of exchange (Swift and Connick, 1962). However, in systems containing paramagnetic ions the nuclear magnetic moments are influenced by the magnetic moment of the paramagnetic species. The NMR linewidths may then have some dependence on the electron relaxation. An understanding of electron relaxation mechanisms is then useful in interpreting the data.

An understanding of electron paramagnetic resonance (EPR) linewidths is importart both in understanding EPR itself, and in interpreting complicated EPR spectra. The EPR linewidths may also be used to study ligand exchange reactions, and to study exchange narrowing effects in concentrated solutions. The EPR linewidths of so-called spin-labels are proving to be important for the study of macromolecules.

The present study was undertaken to extend our knowledge of two important spin $1 / 2$ systems: hexaquocopper (II), $\mathrm{Cu}\left(\mathrm{H}_{2} \mathrm{O}\right)_{6}^{2+}$, and pentaquooxovanadium (IV), $\mathrm{VO}\left(\mathrm{H}_{2} \mathrm{O}_{5}^{2+}\right.$. Past studies of these ions have provided some qualitative understanding of the relaxation effects. However, a complete quantitative interpretation was not possible with the available data. Only dilute solutions are studied so that exchange effects are unimportant. 
Both ions are also rather stable ir aqueous solution, and their perchlorate salts will give these ions in solution without further complications. The measurement of the linewidths in these systems was greatly ficilitated by the development and use of a digital data acquisition systel. Indeed, the measurements of the hexaquocopper (II) complex would not have been feasible without this system. The data acquisition system operated in an "off-line" mode with the digitized spectra stored on the magnetic tape for later analysis by a digital computer. The successful operation of this system was conducive to the development of an "on line" digital computer system. An "on-line" digital computer system has been developed to measure spin-lattice relaxation times from pulse saturation experiments This laboratory has been interested in $\mathrm{Ni}^{+2}$ ions in various lattices for some time (Batchelder, 1970; and Jindo, 1971) and preliminary measurements of $\mathrm{T}_{1}$ for $\mathrm{Ni}^{2+}$. in lanthanum magnesium nitrate single crystals have been performed. 
II. THEORIES OF ELECTRON .SPIN RELAXATION IN SOLUTION

In this section the results of the various relaxation theories for electron paramagnetic resonance (EPR) of spin $1 / 2$ systems in dilute solution are presented. The theories which are specific to systems with spin greater than $1 / 2$ or which apply to concentrated solutions are not presented. The results will not be derived in detail. The reader is referred to the original papers for detail or to the general discussions which are availab]e. Two excellent reviews of relaxation in solution have appeared recently: Hudson and Luckhurst (1969) and Lewis and Morgan (1968). In addition a number of discussions of the general techniques have appeared: Fraenkel (1967); McClachlan (1964); Carrington and Luckhurst (1964).

The first treatment of magnetic resonance relaxation in liquids was due to Bloembergen, Purcell and Pound (1948). Although the original derivation was for nuclear magnetism, the method may also be used in electron paramagnetism. BPP considered the relaxation of: protons in solution. The perturbation causing the relaxation was a magnetic dipole interaction which fluctuated in time due to random thermal motions in solution. BPP used time dependent perturbation theory to compute transition probabilities which could be directly related to the relaxation times. However, they were forced to use the correlation function methods of the theory of Brownian motions since the perturbation was random rather than periodic. The correlation functions were related to spectral densities which were then related to relaxation times. Yariv and Louisell (1962) have also applied perturbation theory to lineviaths. 
More elegant methods for computing relaxation times have since been developed. The best known methods are the linear responsa theory of Kubo and Tomita (1954), and the relaxation matrix theory of Redfield and of Wangsness and Bloch (Redfield, 1957, 1965; Wangsness and Bloch, 1953; Bloch, 1956, 1957). Both of these methods apply the technique of density matrices to the computation of relaxation times.

Although both methods involve the same assumptions and generally arrive at the same answers, the Kubo and Tomita method has not come into widespread use (Deutch and Oppenheim, 1968). The theory has mainly been applied by Kivelson (1957, 1960).

The relaxation matrix method has been widely used in relaxation theories. In this method the relaxation matrix is computed from the correlation functions derived from the perturbation Hamiltonian. The relaxation times are obtained from the components of the relaxation matrix. The method is discussed in detail in Abragam (1961) and in Slichter (1963). The method has been extended and applied by Feed and Fraenkel (1963).

The additional theories which have appeared, and which are extensions of the relaxation matrix theory, have not yet become popular: Fulton (1964); ArE:yres and Kelley (1964); Freed (1968); Sillescu and Kivelson. (1968).

The procedure for the computation of a linewidth or a relaxation tine begins with the assumption of a mechanism or time dependent interaction which could cause relaxation. The next step in the development of the theory is the formulation of a time dependent term (consistent with the mechanism) in the Hamiltonian for the system. The derivation of a mathematically tractable form is perhaps the most difficult part of the theory. 'l'he rinal, though by no means trivial, step is the application of ore of 
general time dependent perturbation methods to compute the relaxation times.

\section{A. Anisotropic $g$ and A Tensors}

The relaxation theory of anisotropic systems is probably the most important theory for transition metal complexes with spin 1/2. The essential ideas behind the anisotropic theory were first proposed by McConnell (1956). The basic idea was that the transition metal complex existed as a stable microcrystallite in solution. The complex therefore possessed the same anisotropic spin Hamiltonian which it would possess in the solid state. The microcrystaliine complex could. tumble in solution due to Brownian motion. The Zeeman interaction, which is orientation dependent for anisotropic systems, is modulated by the rotation of the complex as it undergoes random thermal motions.

This situation is very similar to the dipolar case treated by BPP. McConnell applied the BPP methods to a complex with axial symmetry and arrived at the following result:

$$
\frac{I}{T_{I}} \approx\left(\Delta \mathrm{g}_{B} \text { Ho }+\mathrm{bm}_{\mathrm{T}}\right)^{2} \mathrm{~h}^{-2} \frac{\mathrm{c}}{1+4 \pi^{2} \nu^{2} \tau_{c}^{2}}
$$

where

$$
\begin{gathered}
\Delta g=g_{\|}-g_{1} \\
b=A_{11}-A_{1}
\end{gathered}
$$

and $g_{\|}, g_{\perp}, A_{\|}$, and $A_{\perp}$ are the principal values of the $g$ and $\Lambda$ tensors, respectively, $\mathrm{H}_{0}$, the resonance fielä corresponding to the microvave frequency, $\nu_{0} ; m_{I}$, the nuclear spin quantum number; and $\tau_{c}$, the correlation time characteristic of tumbling in solution. The correlation time, $\tau_{c}$, 
can be related to the hydrodynamic radius, $r$, of the complex and the viscosity, $\dot{r}$, of the solution by the Stokes-Einstein equation

$$
\tau_{c}=4 \pi n \frac{r^{3}}{3 k T}
$$

where $\mathrm{k}$ is Boltzmann's constant, and $T$, the absolute temperature.

Equation 2.1 gives a linewidth with the folloving characteristics. The linewidths vary with $m_{I}$, the nuclear hyperfine quantum number. Secondly, the linewidths vary with the magnetic field. This is reasonable since the Zeeman interaction is proportional to magnetic field. Finally, the linewidths vary directly with the correlation time, or inversely with the temperature. This is opposite to the behavior usually observed in solid state where the temperature must be decreased to narrow the line.

Examinatior of Eq. 2.1 shows three kinds of terms: a term independent of $\mathrm{m}_{I}$, a term Jinear in $\mathrm{m}_{I}$, and a term quadratic in $\mathrm{m}_{\mathrm{I}}$. The $\mathrm{m}_{\mathrm{I}}$ independent term is characteristic of the anisotropy on the $g$ tensor; the quadratic term is related to the anisotropy of the A tensor; and the linear term is a cros:; term related to both anisotropies. Qualitatively a sample has a strong anisotropy in the A tensor if the spectrum shows a symmetric dependence on $\mathrm{m}_{I}$, and a strong $\mathrm{g}$ tensor anisotropy if the spectrum is not symetric in $m_{I}$.

Later, Kivisison $(1960,1964)$ applied the Kubo and Tomita (1954) method and derived essentially the same result. However, he also showed that the linewidth could be expressed as a sum of secular and nonsecular parts. The seizular parts do not contribute to $\mathrm{T}_{1}$ processes, and $\mathrm{T}_{1}$ may by estimated by using only the nonsecular parts of the theory. 
Wilson and Kivelson (1966a) persormed more extensive computations, retaining cross terms which had been neglected in the previous theory. They concluded that the linewidth must be expressed as a cubic polynomial in $m_{I}$ rather tran a quadratic:

$$
\frac{1}{T_{2}}=\alpha^{\prime}+\alpha^{\prime \prime}+\beta m_{I}+\gamma m_{I}^{2}+\delta m_{I}^{3}
$$

where $\alpha^{\prime \prime}$, the residual width, accounts for contributions from effects not included in the tumbling theory. The coefficients of $\mathrm{m}_{\mathrm{I}}$ are given by Eq. $2.5-2.8$.

$$
\begin{aligned}
& \frac{\alpha^{\prime}}{\tau_{R}}=\frac{4}{4}\left(\Delta \gamma B_{0}\right)^{2}+\frac{3}{40} b^{2} I(I+I)-\frac{1}{30} b \frac{a}{\omega_{0}} \Delta \gamma B_{0} I(I+I) \\
& +u\left\{\frac{1}{15}\left(\Delta \gamma B_{0}\right)^{2}+\frac{7}{40} b^{2} I(I+I)-\frac{1}{30} b \frac{a}{\omega_{0}} \Delta \gamma B_{0} I(I+I)\right. \\
& \left.\therefore \frac{5}{40} b^{2} \cdot \frac{a}{\omega_{0}} I(I+I) f\right\} \\
& +\frac{4}{15}\left(\delta \gamma_{0}\right)^{2}+\frac{2}{5} I(I+I) c^{2}+u\left[\frac{I}{5}\left(\delta \gamma_{0}\right)^{2}+\frac{I 4}{15} I(I+I) c^{2}\right] \\
& \frac{B}{\tau R}=\frac{4}{15} \quad b \Delta \gamma B_{0}-\frac{8}{45}\left(\Delta \gamma B_{0}\right)^{2} \frac{a}{\omega_{0}}-b^{2} \frac{a}{\omega_{0}}\left[-\frac{1}{20} I(I+I)+\frac{3}{40}\right] \\
& +u\left\{\frac{1}{5}\left[b \Delta \gamma B_{0}-\frac{2}{3}\left(\Delta \gamma B_{0}\right)^{2} \frac{a}{\omega_{0}}(1+f)\right]-\frac{1}{20} b^{2} \frac{a}{\omega_{0}}\right. \\
& x[I(I+1)+I+7 I(I+I) f]\}+\frac{16}{15} c \delta \gamma B_{0}+\frac{4}{5} c \delta \gamma B_{0} u \\
& \frac{x}{\tau_{R}}=\frac{1}{8} v^{2}-\frac{7}{30} v \frac{a}{\omega_{0}} \Delta{ }^{2} B_{0} \\
& -u\left\{\frac{1}{14} b^{2}+\frac{1}{6} b \frac{a}{w_{0}} \Delta \gamma B_{0}+\left(\frac{2}{5} b \frac{a}{w_{0}} \Delta \gamma B_{0}-\frac{5}{40} b^{2} \frac{a}{\omega_{0}}\right) f\right\} \\
& +3 n^{2}-\frac{2}{15} n^{2} u
\end{aligned}
$$




$$
\frac{\delta}{\tau_{R}}=\frac{1}{20} b^{2} \frac{a}{\omega_{0}}+\frac{1}{20} b^{2} \cdot \frac{a}{\omega_{0}} u(1+f)
$$

where

$$
\begin{array}{ll}
b=\frac{2}{3}\left[A_{z}-\frac{1}{2}\left(A_{x}+A_{y}\right)\right] & \Delta y=B \Delta g / h \\
\Delta g=B_{z}-\frac{1}{2}\left(B_{x}+g_{y}\right) & \delta_{g}=\frac{1}{2}\left(E_{x}-E_{y}\right) \\
c=\frac{1}{4}\left(A_{x}-A_{y}\right) & B_{0}=\hbar \omega_{0} / g \beta \\
u=1 /\left(1+\omega_{0}^{2} \tau_{R}^{2}\right) & \delta \gamma=B \delta g / h \\
f=\omega_{0}^{2}{ }^{2}{ }_{R}^{2} u
\end{array}
$$

In the axial case all of the terms containing $c$, or or vanish. Furthermore, we have

$$
\begin{gathered}
\Delta g=B_{\|}-B_{1} \\
b=A_{11}-A_{1}
\end{gathered}
$$

Qualitatively, the linewidth has the same characteristics as the McConnell linewidth. However, the detailed behavior is much more complicated. The coefficients no longer have a simple interpretation in terms of a given anisotropy but include cross terms (however, the najor contribution from a given anisotropy to a given coefficient is as discussed above). The temperature dependence of the lincwidth is also slightly changed.

These results have also been derived by other workers using the relaxation matrix theory: Hudson and Luckhurst (1969); McClachlan (1964). Sames (1967) has also derived similar results.

\section{B. Spin-Rotation Interactions}

As a molecule tumbles in solution, the rotating electron cloud of the molecule produces a magnetic dipole moment. This moment can interact 
with the nuclear and the electronic spins in the molecule. This interaction can be considered as an interaction of the spin angular momenta with the rotational angular momentum of the molecule. . The problem was treated for nuclear relaxation by a number of workers, notably Hubbard (1963).

Hubbard considered molecules with cylindrical symmetry and obtained

$$
\mathrm{T}_{1}^{-1}=\mathrm{T}_{2}^{-1}=\left(2 \mathrm{ckT} \tau_{\omega} / 3 \hbar^{2}\right)\left(2 \mathrm{C}_{1}^{2}+\mathrm{C}_{\|}^{2}\right)
$$

where $C_{\|}$and $C_{1}$ are the principal values of the spin-rotation interaction tensor; $\tau_{\omega}$, the correlation time characteristic of the spin-rotation interaction, and of the molecular moment of inertia. The correlation time $\tau_{\omega}$, is not the same as the correlation time for the tumb] ing process and is given by

$$
\tau_{\omega}=\varphi /\left(8 \pi r^{3} n\right)
$$

where $r$ is the hydrodynamic radius and $n$ the viscosity. The spinrotation linewidth is then independent of magnetic field and proportional to $\mathrm{I} / \mathrm{n}$. In contrast the anisotropic contribution of $\mathrm{Eq} \cdot 2.1$ is usually proportional to $\mathrm{n} / \mathrm{T}$.

The theory is difficult to apply in this form and was extended by Atkins and Kivelson (1966) for the problem, in EPR. They related the electron spin-rotation tensor, $C$, to the $g$ tensor and derived a linewidth

$$
\mathrm{T}_{2}{ }^{-1}=\left(12 \pi \mathrm{r}^{3}\right)^{-1}\left(\Delta \mathrm{g}_{\|}^{2}+2 \Delta \mathrm{g}_{1}^{2}\right) \mathrm{kT} / \mathrm{n}
$$

where

$$
\begin{aligned}
& \Delta g_{\|}=g_{\|}-2.0023 \\
& \Delta g_{\perp}=g_{1}-2.0023
\end{aligned}
$$


The linewidth is now independent of the moment of inertia of the molecule. The spin-rotation mechanism should be distinguished from the rotationalspin-orbit mechanism which will be discussed later.

Wj.Ison and Kivelson (1966c) and McClung and Kivelson (1968) have shown that in many cases the hydrodynamic radius, $r$, must be replaced by

$$
r=k r o
$$

where $K$ is a constant which depends upon the solvert and its interaction with the solute, and $r_{0}$ is a radius determined by other measurements or by calculation of the molecular dimensions. The radius, $r_{0}$, is a constant characteristic of the molecule and may be determined from translational diffusion measurements. The parameter, $K$, is related to the anisotropy of the intermolecular potential energy between the solvent and the solute, and is expected to be small for an isotropic interaction. It is expected to be large, i.e., $\mathrm{K} \sim \mathrm{I}$, for $\mathrm{H}_{2} \mathrm{O}$ and hydrogen bonded solutes. The usual Stokes-Einstein equation should be adequate in aqueous solution wilh solutes (complexes) which are hydrogen bonded.

\section{Al'tshuler and Valiev Mechanism}

Al'tshuler and Valiev (1958) have proposed a relaxation mechanism for solutions which should be applicable to isotropic systems as well as to anisotropic systems. In contrast to the McConnell tumbling mechanism which is produced through rotational modulation of the spin Hamiltonian, the Al'tshuler and vallev mechunisis is produced by vibrational modulation.

In accord with the Van Vleck (1939) development for solid state, the complex ịs no longer regarded as a rigid molecule which can only rotate; the ligands around the central ion are permitted to vibrate. As a result 
the crystalline potential field of the complex, which is determined by the configuration of the ligands, is modulated. Spin relaxation may be produced through spin-orbit interaction.

The vibrational modulation is introduced into the spin Hamiltonian through the potential energy which is expressed as an expansion in the normal coordinates of an octahedral complex (Van Vleck, 1939)

$$
\mathcal{H}^{\prime}=\sum_{i=2}^{6} \mathrm{~V}^{i} \mathrm{Q}_{i}
$$

where $Q_{i}$ is the ith symmetry coordinate of the octahedron, and $V^{i}=\partial V / \partial Q$. The perturbation is then independent of the anisotropy of the complex. The vibrations of the complex are stochastic since the ligands are influenced by the Brownian motions of the surrounding particles. Al'tshuler and Valiev assume an exponential form for the corielation function and derive a transition probability $A_{l k}$ between energy levels $I$ and $k$

$$
A_{I k}=\frac{\overline{Q^{2}}}{h^{2}} \sum_{i} i v_{l k}^{i} \gamma^{2} \frac{2 \tau_{c}}{1+\omega_{I k}^{2} \tau^{2}} \text {. }
$$

$\overline{Q^{2}}$ is a kind of average measure for the amplitude of the nosmal.

vibrations. Al'tshuler and Valiev assume that the mean square amilitude of the oscillator is given by

$$
\left\langle Q^{2}\right\rangle=\left(\hbar / 2 m \omega_{0}\right) \operatorname{coth}\left(\hbar \omega_{0} / 2 \mathrm{kT}\right)
$$

where $\omega_{0}$ is an average frequency for the vibration and $m$ is a mass close to the mass of the complex. To find the temperature dependence of $A_{1 k}$ they further assume that the correlation time, ' $\tau_{c}$ ' is inversely proportional to the square root of the absolute temperature. Hence, they show the temperature dependence of the transition probability to be 


$$
A_{1 k} \sim T^{-1 / 2} \operatorname{coth}\left(\hbar \omega_{0} / 2 k T\right)
$$

for

$$
\tau_{c}^{2} \omega_{i k}^{2} \ll 1
$$

and

$$
A_{1 k} \sim T^{1 / 2} \operatorname{coth}\left(h \omega_{0} / 2 k T\right)
$$

for

$$
\tau_{c}^{2} \omega_{1 k}^{2} \gg 1
$$

Hayes (196i) reconsidered the Al'tshuler and valiev theory and indicatco that two of the ussumptions of the theory are incorrect. First; the spectral density for the random variable is not normalized, but should be since the total, power in the system should not change with the correlation time: Furthermore, the mean square value of $Q^{2}$ is correct only at frequencies far removed from the resonant frequency.

In light of these criticisms Hayes rederived the result for the result for the transition probability with this mechanisin. He concluded that the transition probability should be directly proportional to temperature. However; he comments that the proportionality constant would be very difficult to determine,

In a later work Valiev and 'Zaripov' (1962) reconsidered the original theory. They indicate that a second term, quadratic in the normal coordinates, should be added to the perturbation. Furthermore, the quadratic term should be more effective than the linear term in producing relaxation: The original theory produced reasonable results because of the form assumed for the correlation functions. Valiev and Zaripor revise the correlation functions in accord with Hayes. Their result for the transition probability cannot be presented in a simple form. Howcver, the temperature dependence can be shown to be 


$$
\mathrm{T}_{1}^{-1} \approx \operatorname{coth}^{2}\left(\frac{\hbar \omega_{o}}{2 k T}\right) \frac{\tau_{c}}{1+\omega_{1 k}^{2} \tau_{c}^{2}}
$$

The temperature dependence is similar to the McConnell temperature dependence until $\mathrm{T} \approx \hbar \omega_{0} / 2 \mathrm{k}$. For. $\mathrm{T} \gg h \omega_{0} / 2 \mathrm{k}, \mathrm{T}_{1}^{-1}$ may in some cases increase with increasing temperature.

\section{Inversion Mechanism}

Spencer (1965) has considered a process which is applicable to complexes which have a number of equivalent ground state configuraticns, such as Jahn-Teller systems. In particular, an octahełral complex si:ch as $\mathrm{Cu}\left(\mathrm{H}_{2} \mathrm{O}\right)_{6}^{2+}$ may distort tetragonally along either of the equivalent $x$, $y$, and $z$ axes. The result of passage from distortion xlong one axis to distortion along another axis is equivalent to a $90^{\circ}$ rotation of the complex: However, unlike the tumbling mechanism, the complex does $\mathrm{n} t$ pass continually through all possible orientations. The complex "in erts" or "jumps" from one orientation to another.

Spencer describes this process in the spin Hamiltonian by defining special delta functions. The delta functions are one or zero depending upon the orientation. Performing a McConnell type of derivation, Spencer shows that the linewidth contribution, $1 / \mathrm{T}_{2}$, from this mechanism is

$$
\frac{1}{T_{2}^{\prime}}=\frac{32 \pi}{9 h^{2}}\left(\Delta g B H_{0}+b m_{I}\right)^{2} \tau_{i}
$$

where $\tau_{i}$ is the correlation time for inversion, and the other terms have their usual meanings. The contribution from this mechanism should be larger than the tumbiling meshanism, but both mechanisms should have the same kind of dependence on field and $m_{I}$. However, this mechanism 
should have very little contribution to $\mathrm{T}_{1}$ processes, unlike the tumbling process which. has a strong contribution. Spencer does not discuss a temperature dependence for this mechanism. However, we expect $1 / T_{2}$ ' to be proportional to $n / T$.

\section{E. Electric Field Fluctuation Mechanisms}

Kivelson (1966) has considered a number of relaxation mechanisms which he calls electric field fluctuation (eff) mechanisms. In these mechanisms the crystal field of the complex is modulated by vibrations of collisions with surrounding molecules. Spin relaxation occurs through spin-orbit coupling. Among the eff mechanisms are the vibrational spin-osbit, the rotational spin-orbit, the Van Vleck Direct, the Van Vleck Ramin, and the Orbach processes.

Kivelson and Collins (1962) originally considered the vibrational spin-orbit and the rotational spin-orbit processes. The rotational spin-orbit process which is an Orbach type of process is to be distinguished from the spin rotational interaction described above. Relaxation by the rotational spin-orbit process occurs through an excited electronic state in contrast to the spin rotation interaction which does not involve excited statrs. Kivelson and Collins show that the contribution to the linewidth from this mechanism is given by

$$
\left.\mathrm{T}_{2}^{-1}(\mathrm{R}: 0)=4 / 3 \sum_{\mathrm{n}}^{\sum^{\prime}} \sum_{\alpha} \mid k \quad \text { ol } L_{\alpha} \mid \mathrm{n}\right)\left.\right|^{2}\left(\lambda / \Delta_{\mathrm{On}}\right)^{2} \mathrm{~T}_{\mathrm{r}}^{-1}
$$

where $\lambda$ is the spin-orbit coupling constant, $\Delta_{\text {on }}$ the frequency of an electronic tsansition from the ground to the $n$th state, $\tau_{R}$ the rotational. correlation, ime, and $\left(0\left|L_{\alpha}\right| n\right)$ the matrix elements for the orbital anguiar 
momentum operator $\mathrm{L}_{\alpha}$. They compare this to the contribution from the tumbling mechanism and conclude that this is much smaller thar the tumbing mechanism contribution. This mechanism can then be neglected.

The Van Vleck Direct, the Van Vleck Raman, and the Orbach processes are analogous to the solid. state processes for which they were named. The direct process is a one phonon process and the Raman process is a two phonon process involving a virtual excited state. The Orlach process is a two phonon process involving an actual excited state.

The Orbach process, discussed by Kivelson (1966), was celled the vibrational spin-orbit process by Kivelson and Collins (1962). The perturbation in the process is not due to the molecular normal coordjnates, but rather to the lattice or liquid modes, that is, the fluctuations of the intermolecular coordinates in liquids. Kivelson considers the molecular normal coordinates only in a direct vibrational process. His results indicate that the contribution from the vibrational process is negligible. Atkins and Kivelson (1966) also consider the effect of molecular normal coordinates in direct, Orbach, and Raman types of processes. They indicate that the linewidth contribution is negligible.

Kivelson has computed the contribution of the Orbach process in the limit $\delta_{\text {on }} \tau_{c}>1$ to be

$$
\mathrm{T}_{1}^{-1}=\mathrm{T}_{2}^{-1}=16(\lambda / \Delta)^{2}\left(\frac{\phi^{\prime} \mathrm{g}_{\mathrm{o}}}{\Delta \mathrm{r}_{\mathrm{o}}}\right)^{2}\left(\frac{\Delta}{\delta_{\mathrm{on}}}\right)^{2} \frac{\tau_{\mathrm{c}}^{-1}}{\left[\exp \left(\hbar_{\mathrm{on}} / k \mathrm{kT}\right)-1\right]}
$$

where $\Delta$ is the energy from the ground orbital state to the lowest excited orbital static (connected by spin-ortit coupling), $\tau_{s}$ the correlation time churactiristic or the eff process, $\delta_{\text {on }}$ the eneryy of the lowest. 
excited electronic state above the ground state. The term, $\phi^{\prime} q_{0} / r_{0}$, is the measure of the time dependent electric field potential (Lewis and Morgan, 1968) with $q_{0}$ a typical amplitude for a lattice (liquid) modes, $r_{0} a$ characteristic intermolecular distance, and $\phi^{\prime}$ a measure of the change in electric field with respect to the lattice mode. The Orbach linewidth contribution which depends on the phonon spectrum at a frequency not directly related to the. EPR transition in independent of the applied tield. Kivelson also indicates that the very similar process suggested by AI'tshuler and Valiev (1958) does not appear to give spin relaxation.

The contribution of the Van Vleck direct process to the linewidth is given by

$$
\mathrm{T}_{1}^{-1}=\mathrm{T}_{2}^{-1}=64(\lambda / \Delta)^{2}\left(\frac{\phi^{\prime} q_{0}}{\Delta r_{0}}\right)^{2} \frac{\left(\omega_{0} \tau_{c}\right)^{2} \tau_{c}^{-1}}{1+\omega_{0}^{2} \tau_{c}^{2}}
$$

where $\omega_{0}$ is the frequency of the spin transition. If $\omega_{0}^{2} \tau_{c}^{2} \ll I$ the the linewidth contribution is proportional to the applied field squared wh whereas if $\dot{\omega}_{c}^{2} \tau_{c}^{2} \gg 1$, it is independent of the applied field.

Kivelson considered the linewidth contribution from both a first order Raman process and a second order Raman process. The first order process is less important than the second order process. The contribution from the second order process under the condition $\omega_{0}^{2} \tau_{c}^{2} \ll I$ is given by

$$
\mathrm{T}_{1}^{-1}=\mathrm{T}_{2}^{-I}=32(\lambda / \Delta)^{2} \cdot\left(\frac{\phi^{\prime} \mathrm{q}_{0}}{\Delta \mathrm{r}_{0}}\right)^{4} \cdot \tau_{\mathrm{c}}{ }^{=1}
$$

This process is independent of the applied fjeld. 
However, in the case of a symmetric or nearly symmetric molecule the contribution from the Orbach process could be quite significant. The contributions from the direct and the Raman processes seem to be quite small. 


\section{VANADYL LINEWIDTHS}

\section{A. Introduction}

The EPR spectrum of the oxovanadium (IV.) ion (vanadyl ion) ${ }^{*}$ in aqueous solution has been known for many years. It was first reported by Garif'yanov and Kozyrev (1954) in Russia and by Pake and Sands (1955) in this country. The spectrum exhibited eight hyperfine lines arising from an interaction with the nuclear spin $I=7 / 2$ of the vanadium-5I nucleus. 'l'he interesting feature of the spectrum was that the linewidths varied with the nuclear quantum number, $\mathrm{m}_{I}$. The lines also narrowed as the temperature was increased.

The Kivelson (1957, 1960) extension of the McConnell (1956) theory was applied to explain the linewidths. The theory has been found to be quite successful in explaining the linewidths in spin $1 / 2$ complexes and many complexes with vanadyl are well characterized by the Kivelson theory. However, al.though the vanadyl system is now used as a prime example of the success of the McConnell mechanism, the pentaquo-coordinated vanadyl ion itself has been very little studied (Lewis and Morgan, 1968).

The first confirmation of the Kivelson theory for the vanadyl system wäs reported by Rogers and Pake (1960). These workers measured the EPR spectra for aqueous solutions of the vanary inn at 9.25 GHz (X-band) and at $24.3 \mathrm{GHz}$ (K-band). Using the $\mathrm{X}$-band spectrum they determined the coefficients of the Kivelson linewidth polynomial which they wrote as

$$
\frac{1}{T_{2}}=\pi \sqrt{3}\left(\alpha_{1}+\alpha_{2} m_{I}+\alpha_{3} m_{I}^{2}\right)
$$

* Strictly, vanadyl (IV)ion (Selbin, 1965). In this work the term vanadyl. will be used to represent vanadyl (IV), vo2t. 
where the coefficients are given by.

$$
\begin{aligned}
& \alpha_{1}=\tau_{c}\left\{7 / 45\left(\Delta \gamma B_{0}\right)^{2}+63 \cdot b^{2} / 3.6\right\}+K \\
& \alpha_{2}=-\tau_{c}\left\{7 / 15 b \Delta \gamma B_{0}\right\} \\
& \alpha_{3}=\tau_{c}\left\{b^{2} / 10\right\}
\end{aligned}
$$

In these expressions $K$ is a constant accounting for linewidth not explained by the theory. The other variables are as defined previously. From their $\mathrm{X}$-band measurements Rogers and Pake predicted the $\mathrm{K}$-band Iinewidths. The predicted widths were in relatively good agreement with the measured widths.

However, this work was unable to provide a quantitative check of the Kivelson theory. The authors lacked the anisotropic spin Hamiltonian parameters recessary to compute the Kivelson parameters. They also did not have a value for the correlation time, and neglected higher order terms in the Kivelson theory.

In a later work, 11cCain. $(1966,1967)$ extended the study of the vanadyl system. He determined the anisotropic spin Hamiltonian parameters for the vanadyl ion from a glass spectrum. He also measured the solution EPR spectra at $3.12 \mathrm{GHz}$ ( $\mathrm{S}-$ band) as well as at $9.07 \mathrm{GHz}$. From the spectra he determined experimental values for the Kivelson coefficients in a polynomial which he wrote as* ${ }^{*}$.

$$
\frac{1}{\mathrm{~T}_{2 \mathrm{e}}}=\alpha_{2}^{\prime}+\alpha_{2}+\beta_{2} \mathrm{~m}+\gamma_{2} \mathrm{~m}^{2}+\delta_{2} \mathrm{~m}^{3}
$$

He also computed theoretical values for these coefficients. However, he was forced to assume a correlation time for his computations. His comparisons indicated reasonable agreement between the theoretical and experimental terms for all the parameters except the alpha term. His

\footnotetext{
* The subscript e emphasizes that this is an electronic as opposed to the nuclear linewidth.
} 
results indicated a $30 \%$ discrepancy at $X$-band and an even larger discrepancy at S-band. Some of this error could be attributed to the uncertainty in the correlation time and some to terms which were neglected in the theory.: Another mechanism, the spin-rotation mechanism, could also account for part of the discrepancy.

Lewis and Morgan. (1968) later reconsidered these results. They assumed a correlation time and computed both the tumbling and spinrotation contributions to alpha. Their estimate of 9.4 gauss is 3.3 gauss less than the value of alpha which McCain observed. They suggested that the residual width was due to superhyperfine interaction with the protons of the water ligands. They predict a hyperfine coupling constant of about $2.2 \mathrm{G}$.

However, in another study Garif'yanov, et al. (Garif'yanov, Kozyrev, Timerov and Usacheva, 1962b) studied the EPR of dilute vanadyl chloride solutions at different temperatures and viscosities. They concluded from their data that, although the McConnell mechanism contributed to the linewidth of vanadyl solutions, it was not the dominant relaxation mechanism. Instead, they suggested that the Al'tshuler and Valiev (1958) mechanism was the most probable IIne broadening mechanism:

The present study was begun to clarify the'situation in the vanadyl system. Moreover, McCain (1967) hạd also determined relative T $T_{1 e}$ values for the S-band spectrum using an ingenious saturation method. Using the nonsecular parts of the Kivelson theory he also computed theoretical values for the relative $\mathrm{T}_{\mathrm{l}} \mathrm{I}^{\prime} \mathrm{s}$. His comparisons indicated that the theory was not perfect. However, he did not have a good value for the correlation time. These results are reconsidered in this work. 


\section{B. Experimentel Methods}

\section{Samples}

All aqueous solution experiments were performed using samples of vanadyl perchlorate which were made in one of the following methods. In the first method, due to Selbin and Holmes (1962), a solution of vanadyl. sulfate was treated with an equivalent amount of a solution of sodium hydroxide. The precipitate of vanadyl hydroxide was filtered and then dissolved in the necessary amount of perchloric acid. The solution of vanadyl perchloratie was then adjusted to the proper concentration.

In the second method vanadyl perchlorate was prepared by ion exchange using the following procedure (Lee et. al., 1968). An exchange column was loaded with a slurry of a cation exchange resin (AG50W-X2, 200400 mesh, hydrogen form) in $2 \mathrm{M} \mathrm{HCl}$. The column was then rinsed with distilled water to remove acid. A solution of vanadyl sulfate was then added to the column and the column was rinsed to rcmove sulfuric acid. A solution of barium perchlorate was used to elute the vanadyl perchlorate from the column. The separation is quite clean anc may easily be followed by observing the blue color characteristic of the ranadyl ion move down the column.

In all cases the solutions were adjusted to a concentration between .01 and $.02 \mathrm{~F}^{*}$ The solutions were made slightly acidic with perchloric acid to inhibit the decomposition of the vanadyi perchlorate. Both methods of preparation were found to be satisfactory for this work.

\footnotetext{
* Formula wẹights per liter:
} 
Solutions of vanadyl ion in $\mathrm{D}_{2} \mathrm{O}$ were made by evaporating a stock solution of agleous vanadvi ion almost to dryness and then dissolving the residue in heavy water. This process was repeated several times to insure a completely deuterated solution. The solutions were never evaporated completely to dryness in order to prevent decomposition from occurring. Deuterated perchloric acid, used in some of the experiments, was obtained Lu a similar manner.

\section{Aviaratus}

EPR spectra were recorded using a standard Varian V-4502 homodyne EPR spectrometer operating at $9.2 \mathrm{GHz}$. Samples for linewidth studies were held in a V-4548 aqueous solution sample cell. This cell is specially designed to confine the sample to a thin plane, thus eliminating line distortion problems which could arise due to solution conductivity. The sample temperature was controlled with a V-4557. Variable Temperature Accessory which controlled the temperature of a stream of nitrogen gas. Temperature was measured with a copper-constantan thermocouple which was placed in the gas stream before or after each experiment in the same position as the sample.

The spectra were digitized using a Honeywe $11-E \perp$ Model s6114 Automat1c Data Logging System (see Fig. 1). This system consists of a HewlettPackard Model 5245L electronic counter, a Honeyvell-EI Model 630s multimeter, a Honeywell-EI Model 825 Output Control Unit, and a Kennedy Model 1400 incremental magnetic tape recorder.

The multimeter (âfital voltmeter) niedsures the EPR signal from the $100 \mathrm{KHz}$ phase detector. The electronic counter measures the magnetic field. In the Varian spectrometer a voltage proportional to the magnetic 


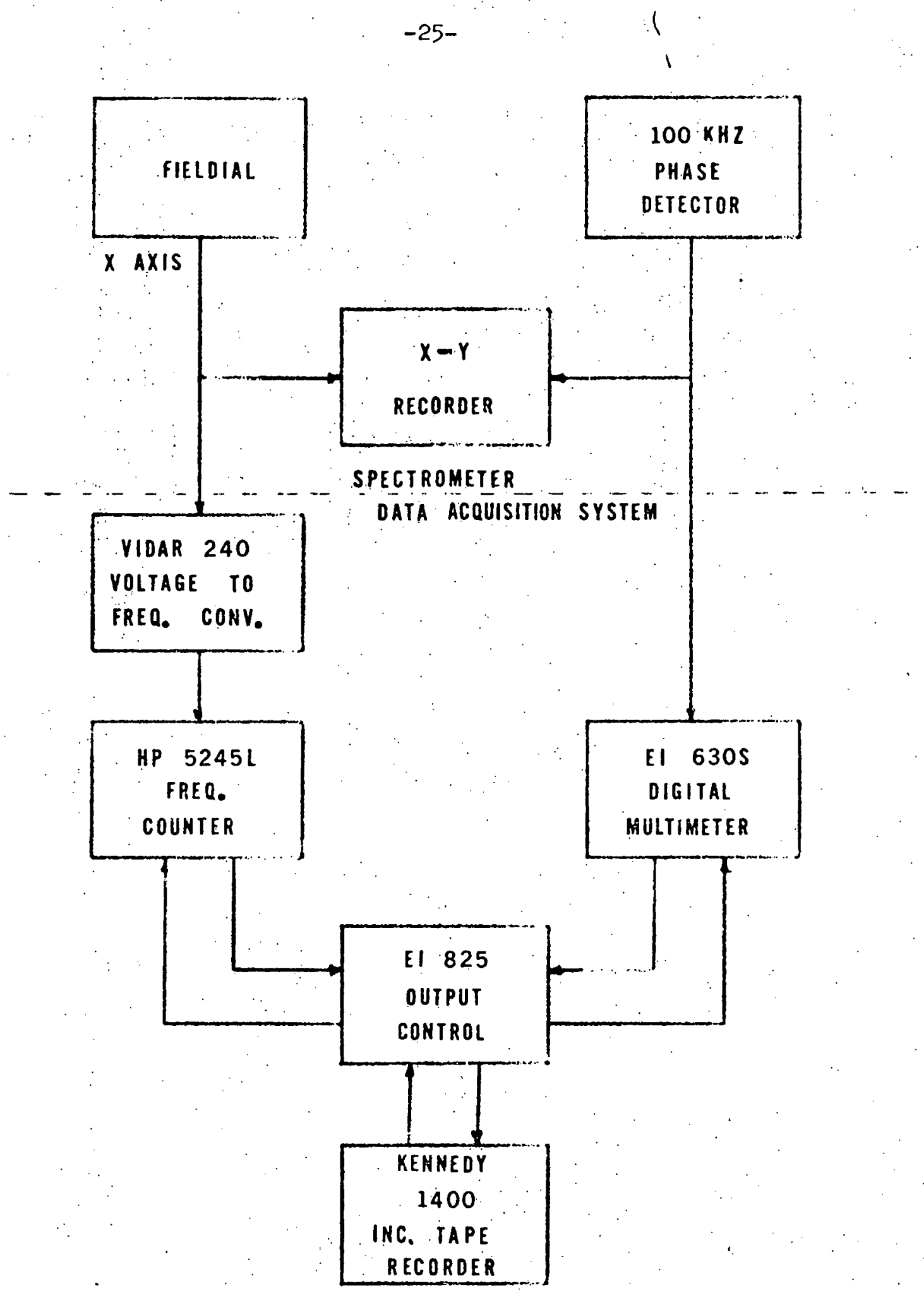

Fjg. 1. Schematic of data acquisition system. 
field is obtained from the $x$-axis retransmitting potentiometer of the Fieldial. This voltage, which originally varied from 0 to 15 volts in order to drive an $x-y$ recorder, was changed to vary from 0 to 10 volts to drive a Vidar Model 240 voltage-to-frequency converter. The electronic counter measures the output from the Vidar and obtains a frequency measurement which can be related to the magnetic field.

The data logging system operates independently of the spectrometer and records the spectrum as a normal scan is in progress. The process of measurement involves simultaneously measuring the EPR signal and the magnetic field coordinates and then encoding and recording the measurement on magnetic tape. The system is capable of making measurements at the rate of 6 to 8 points per second. A digitized spectrum of 2400 points for a normal five minute scan can be routinely acquired with this system.

A measured point consists of seven digits from the frequency counter and five digits plus a sign fron the digital voltmeter. The output control unit adds a "word mark" to form a 14 character word for the measurement. The output unit records the data: in 80 word blocks separated by record gaps on the tape. This is, unforturately, not compatible with the FORTRAN system on most computation centers. The data tape is preprocessed with a special program, SUMTAP (see appendix), before the actual analysis is performed. I'he SUMIAP program puts the data into a form compatible. with the FORIRAN system, smooths the data to relluce noise, and reduces the number of data points in the spectrum for conrenience in handling by the analysis programs. SUMTAP can also average several spectra to improve signal to noise.

The magnetic field is calibrated by measuring both the magnetic field and the Vidar frequency at several points in the sweep. The 
magnetic field was measured with a marginal oscillator (Harvey-Welis 0-502) equipped with a proton probe. The magnetic field may be computed at any point from the Vidar frequency by using a quadratic interpolation equation which is fit to the calibration points. The microwave frequency was measured with an HP $5245 \mathrm{I}$ frequency counter equipped with a HP

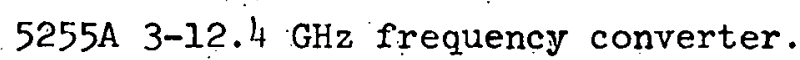

\section{Discussion and Results}

\section{Spectra}

Before a proper treatment of linewidths can be made, the anisotropic spin Hamiltonian parameters, which are utilized in the relaxation theories, must be known. Unfortunately, it is not possible to determine anisotropic parameters from solution (isotropic) spectra, and the se parameters must be determined from solid state measurements. There is, however, no guarantee that the complex which exists in a crystal lattice is the same complex in solution. Distortions often occur in a crystal lattice which do not occur in sclution. Hence, it is not clear that the anisotropic parameters determined from a crystal lattice bear a close relationship to those for a complex in solution, but these are the best parameters which are available.

Although solid state parmeters are best determined from single crystal measurements, single crystals are not readily available for many transition metal complexes. Turthermore, it is usualiy necessary to obtain a crystal with the paramagnetic species diluted in a diamagnetic host lattice in order to avoid dipole-dipole broadening effects. In some cases, the parameters may be determined from polycrystalline or glass spectra. This method was originally used by Sands (1955). 
The methods for analyzing polycrystalline spectra have been well developed (Blinder, 1960; Gersmann and Swalen, 1962; Tbers and Swalen, 1962; Johnston and Hecht, 1965; Kneubuhl, 1960; Neiman and Kivelson, 1961; Vanngard and Aasa, 1963). The spectrum of a polycrystalline sample consists of an envelope of absorption lines rather than distinct single lines. The anisotropic parameters, for example, $g_{\|}$and $g_{1}$ in an axial case, are determined from the extrema of the absorption envelope.

If hyperfine structure is present, the spectrum can be quite difficult to assign. This is especially true if the parameters are such that the spectral features are severely overlapped. In these cases a detailed study of the lineshape is useful. A digital computer can be used to simulate the theoretical absorjtion spectrum. The spectral parameters may be adjusted until satisfactory agreement between the theoretical and experimental spectrum is obtained. The parameters for quite complex spectra can be determined by this technique.

'l'he major problem with polycrystalline spectra seems to be the production of a usable glass. Frozen aqueous solutions are not suitable due to aggregation of the solute and dipolar broadening (Ross, 1965). J.t is usually necessary to use sol.vent mixtures in order to ohtiain a useful. glass medium and many such mixtures have been developed (Smith, et al., 1962). These media have an additional problem in that the solvent may complex with the solute. Spencer (1965) has developed a technique of using $5.26 \mathrm{~F}$ perchloric acjid as a glass medium. A solution of the transition metal complex in perchloric acid can be quick frozen to near jiquid nitrogen temperature to obtain an excellent glass. The advantage of the perchloric acid medium is that the perchlorate anion does not appear to form complexes with transition metals. 
TABLE I Anisotropic Magnetic Parameters for Vanady 1

\begin{tabular}{|c|c|c|c|c|c|}
\hline Solute/Solvent & $g_{\|}$ & $8_{1}$ & $A_{11}^{*}$ & $A_{1}^{*}$ & Reference \\
\hline $\mathrm{VOCl}_{2} /$ Methanol & 1.92 & 1.98 & 190 & 82 & Garif'yanov and Usacheva \\
\hline $\mathrm{VOSO}_{4} / 5.26 \mathrm{~F} \mathrm{HClO}_{4}$ & 1.9312 & 1.9778 & 205.4 & 76.5 & McCair (1967) \\
\hline $\mathrm{VO}\left(\mathrm{ClO}_{4}\right)_{2} / 5.26 \mathrm{~F} \mathrm{HCIO} 4$ & 1.9311 & 1.9785 & 203.3 & 75.8 & This rork \\
\hline${ }^{*}$ Coupling constants ir & n gauss & & & & \\
\hline
\end{tabular}




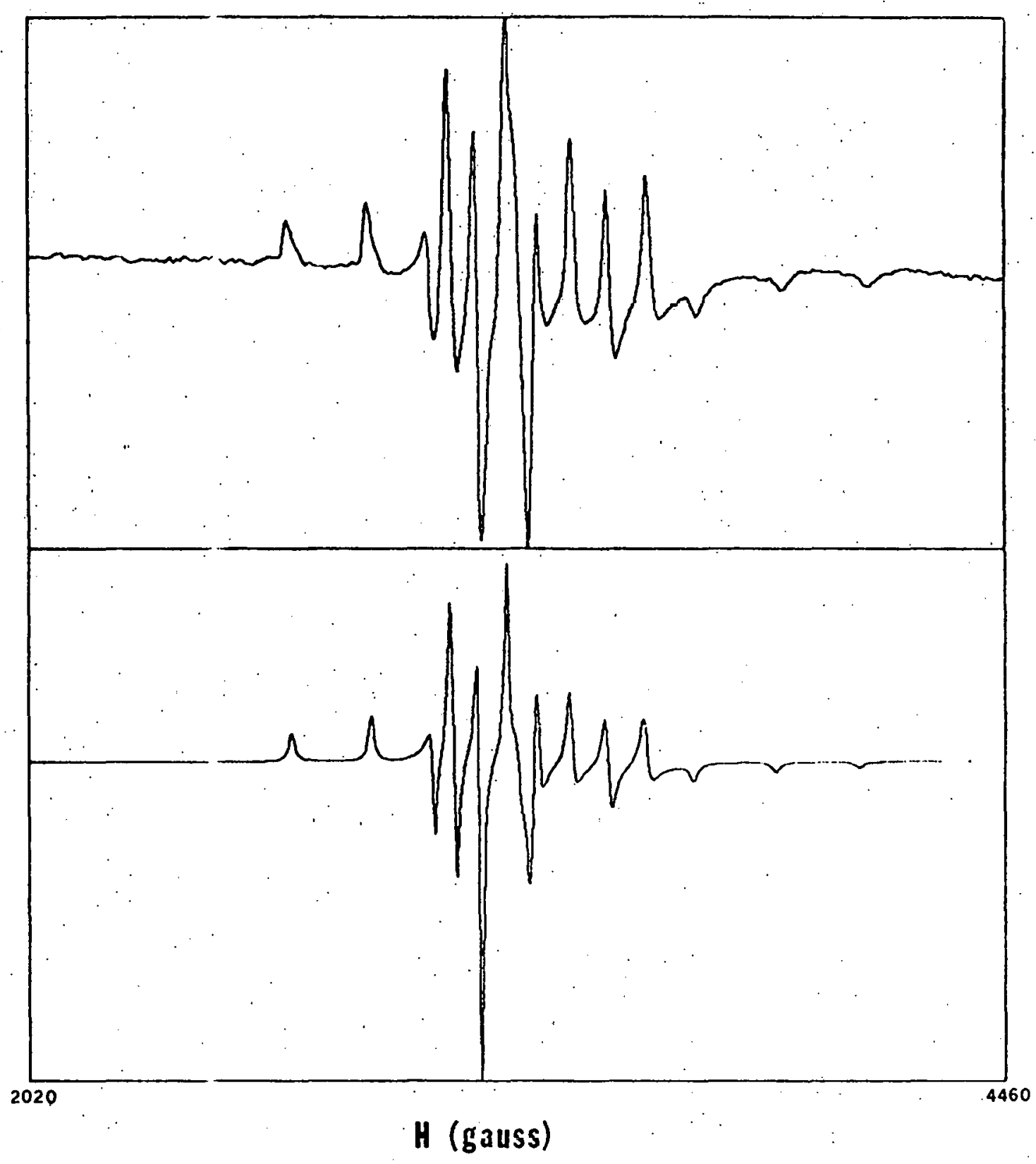

$X B L / U 12-i 16 i$

Fig. 2. $\quad$ Vo $\left(2_{2}\right)_{5}^{2+}$ glass spectrum. Upper spectrum is experimental; lower spectrum is simulation. 
The anisotropic parameters for $\mathrm{VO}\left(\mathrm{H}_{2} \mathrm{O}\right)_{5}^{2+}$ have been determined from spectra of perchloric acid glasses. The glass spectra were analyzed by: spectrum simulation with the method of Vanngard anc Aasa (1963) (see Fig. 2). The vanadyl parameters are presented in Iable I with those determined by other workers for comparison.

The EPR spectra for vanadyl ions in solution were measured at $X$ band at temperatures between -15 and $100^{\circ} \mathrm{C}$ in order to determine the linewidths and the isotropic spin Hamiltonian parareters. The digitized spectra were analyzed by a least squares fitting procedure with the program FITESR which is described in the appendix (Bauder and Myers, 1968). The eight line vanadyl spectrum may be comlletely described with eleven parameters consisting of the spectrum cente:, the intensity, the coupling constant, and eight linewidths. Very gooi values for these parameters are obtained from the least squares fiting procedure. Two paremeters to account for baseline offset and base ine drift are also used. An example of the fit to a vanadyl spectrum is shown in Fig. 3 . The experimental data are plotted as the crosses asd the fitted curve as a continuous line. The bottom curve (error cur e) is the difference between the theoretical and experimental curves, and is useful for judging whether the fit has properly converged.

The vanady $g$ and $A$ value vary as a function of temperature as show in Figs. 4 and 5. This variation, which is similar to that observed for vanadyl acetylacetonate, is thought to be due to changes in solvation. and bonding (Wilson and Kivelson, 1966a). Indeed, the magnitudes of these parameters change in opposite directions as vould be expected from bording theories. Additional mechanisms are mentioned later in the discussion of 
$-32-$

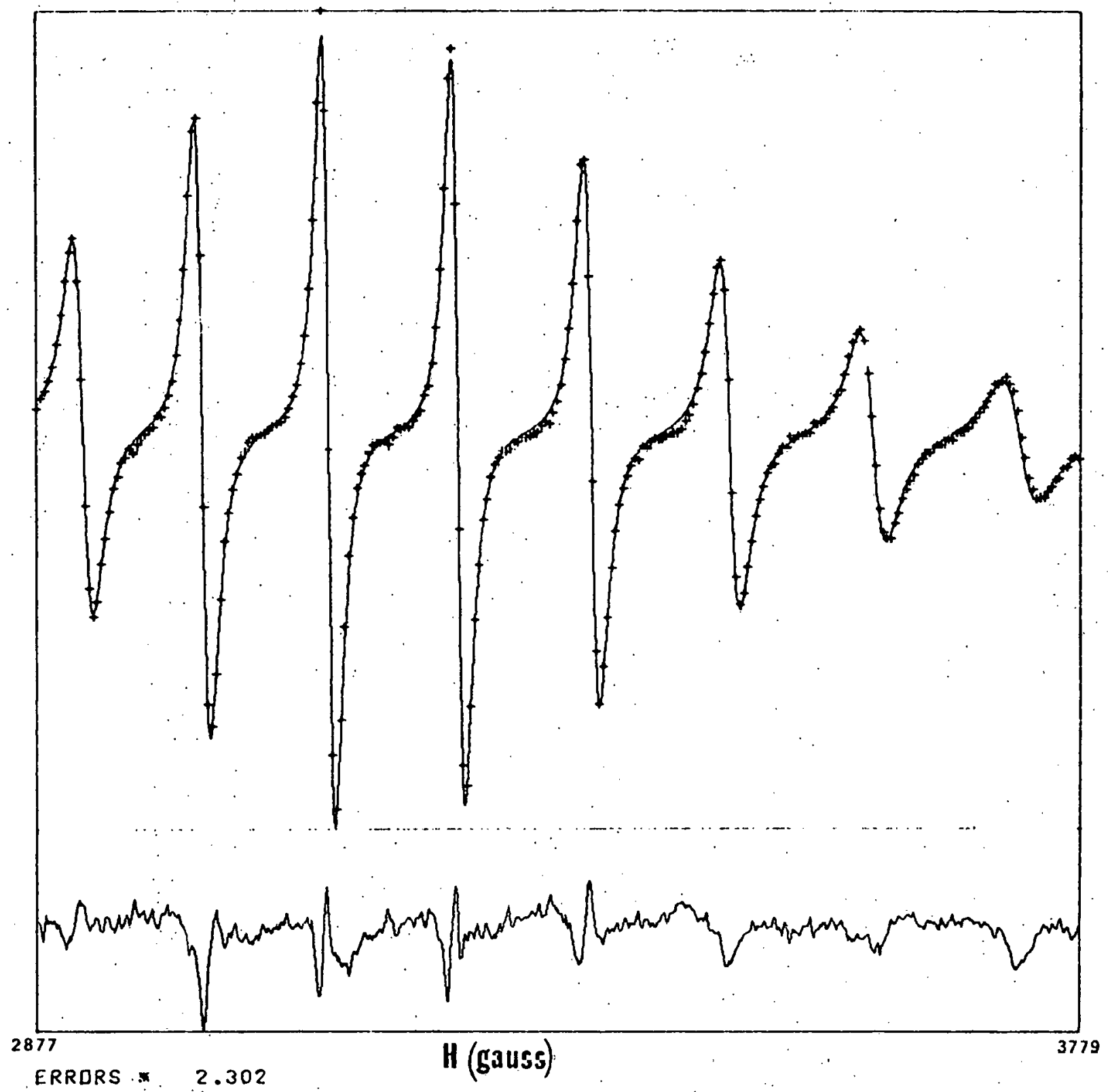

$X B L 7012-7166$

Fig. 3. Example of fit to solution spectrum of $\mathrm{ro}_{2}\left(\mathrm{H}_{2} \mathrm{O}\right)_{5}^{2+}$. The crosses are the experimental points. The continuous line is the themetical fit to the data. The lower curve is the difference between the theoretical and experimental curves and has a scale expansion of 2.3 . 


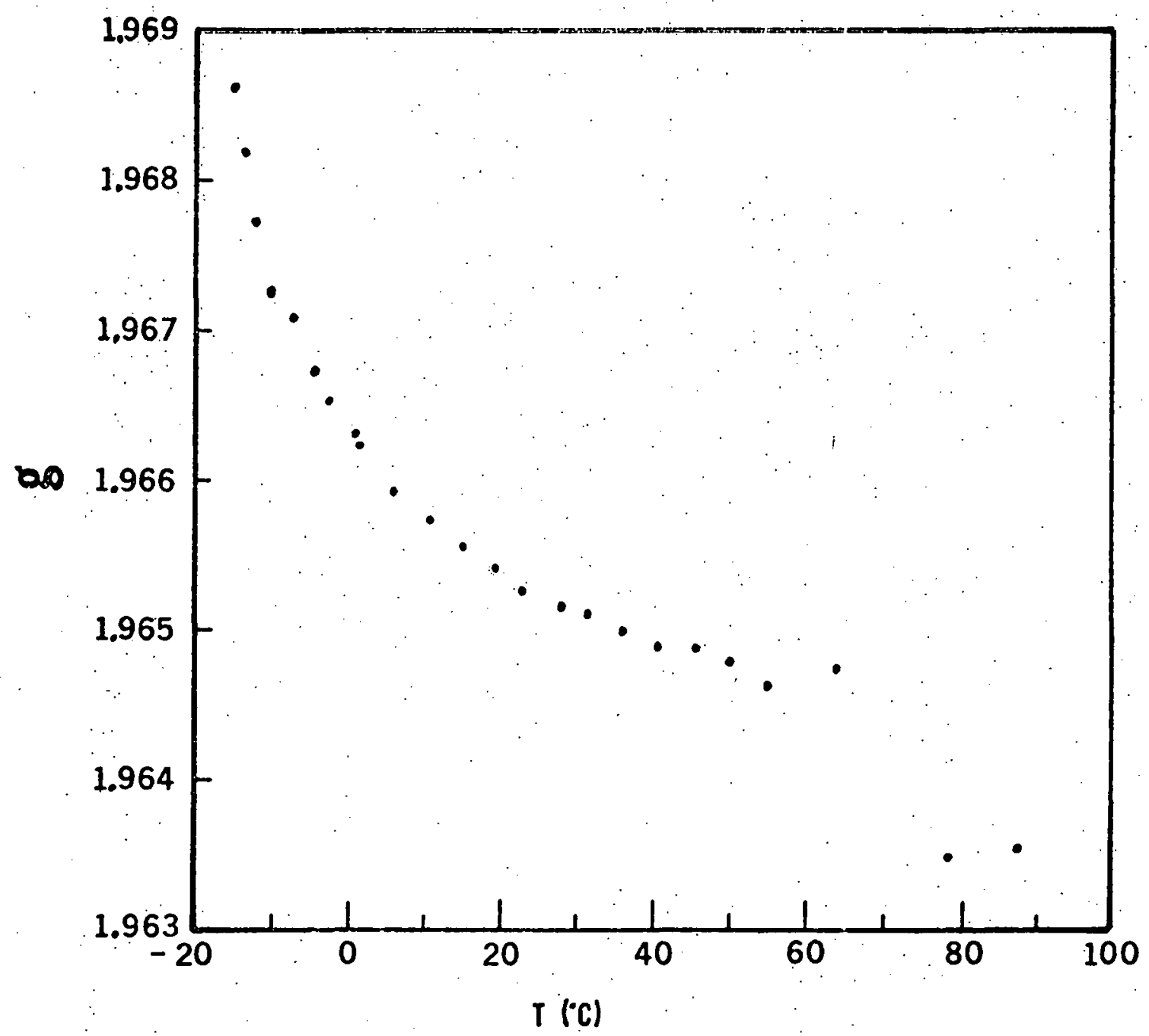

XBL $7012-7349$

Fig. 4. g vs. T for $\operatorname{Vo}\left(\mathrm{H}_{2} \mathrm{O}\right)_{5}^{2+}$ 


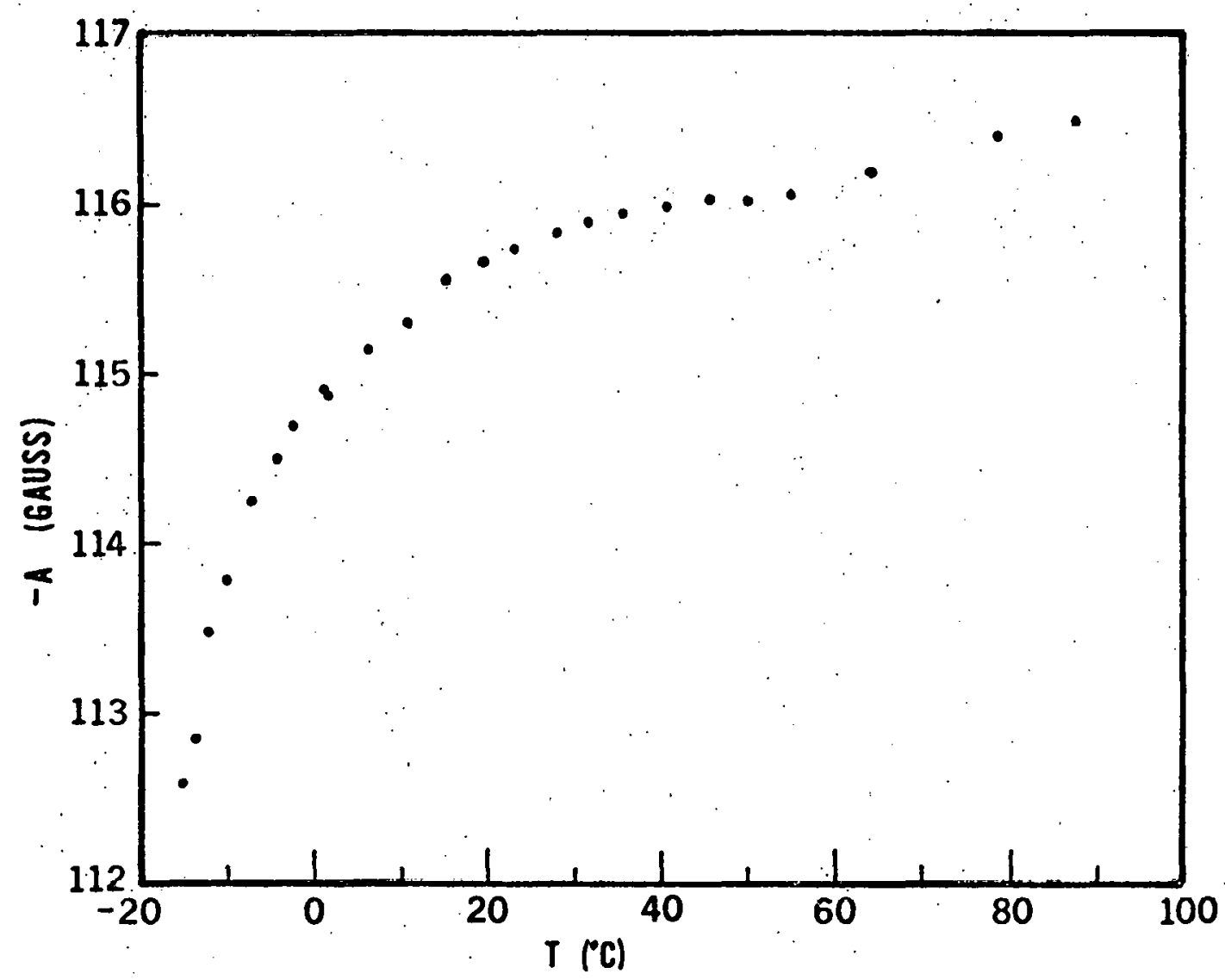

$X B \perp 7012-7348$

Fig. 5. -A vs. T for $\mathrm{VO}\left(\mathrm{H}_{2} \mathrm{O}\right)_{5^{\circ}}^{2+}$ 


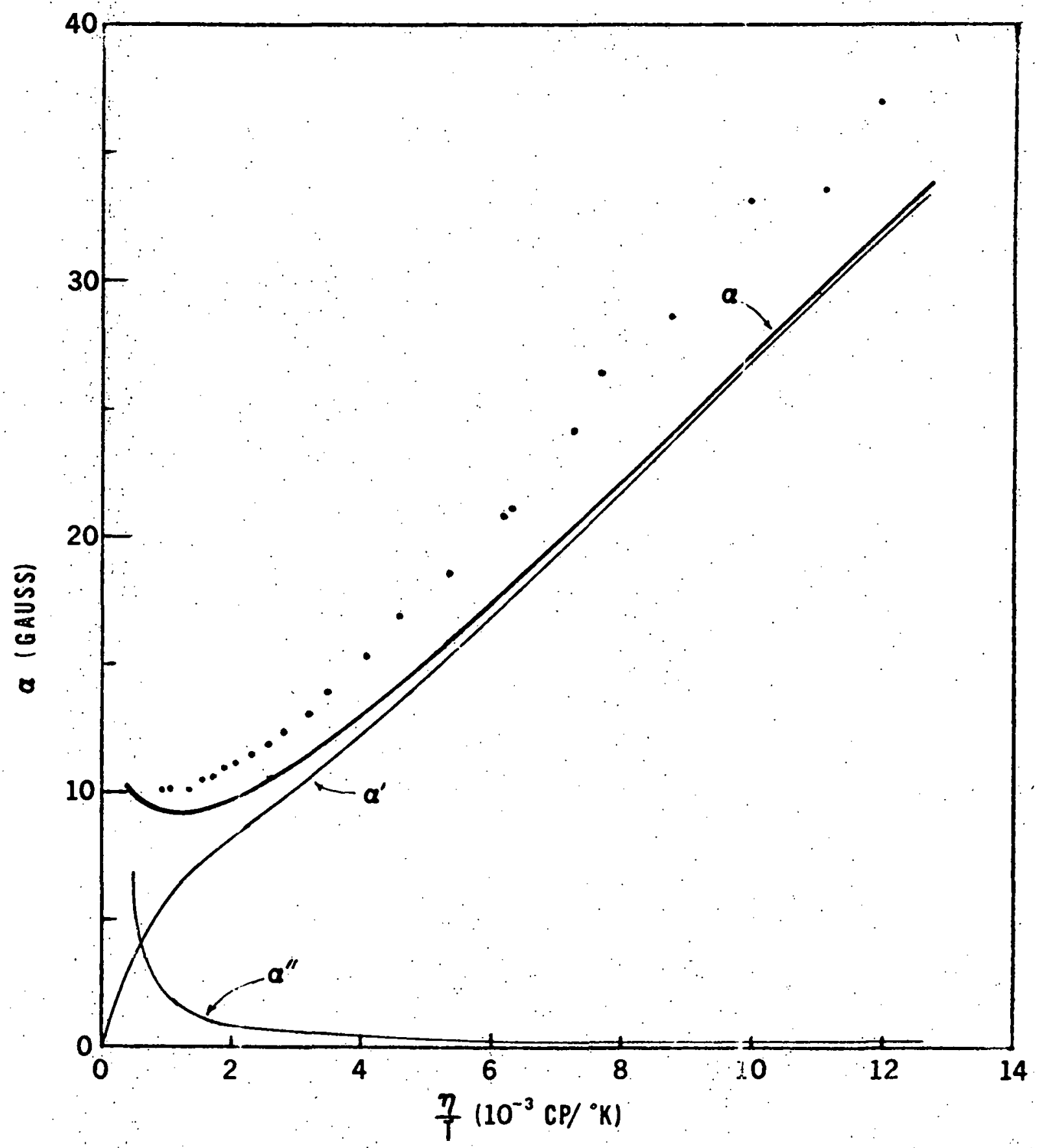

XBL $7012-7342$

Fig. 6. Alpha parameter vs $n / T$ for $\mathrm{VO}\left(\mathrm{H}_{2} \mathrm{O}\right)_{5}^{2+}$ Points are experimental data continuous; Iine is theoretical. 
-36 .

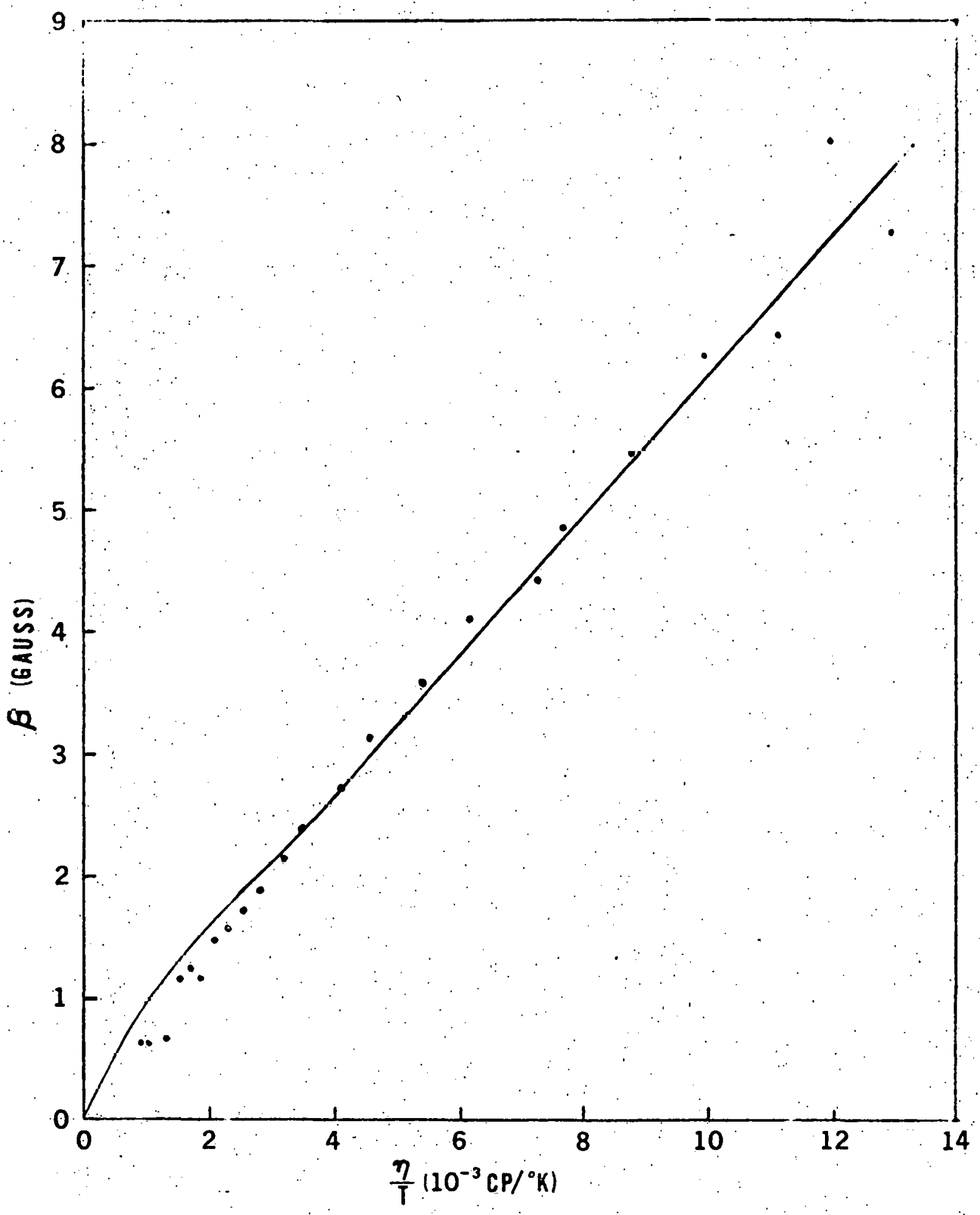

XBL. $7012-7341$

Fig. 7. Beta parameter vs. $n / \mathrm{T}$ for $\operatorname{vo}\left(\mathrm{H}_{2} \mathrm{O}\right)_{5}^{2 .+t}$. 


$$
-37-
$$

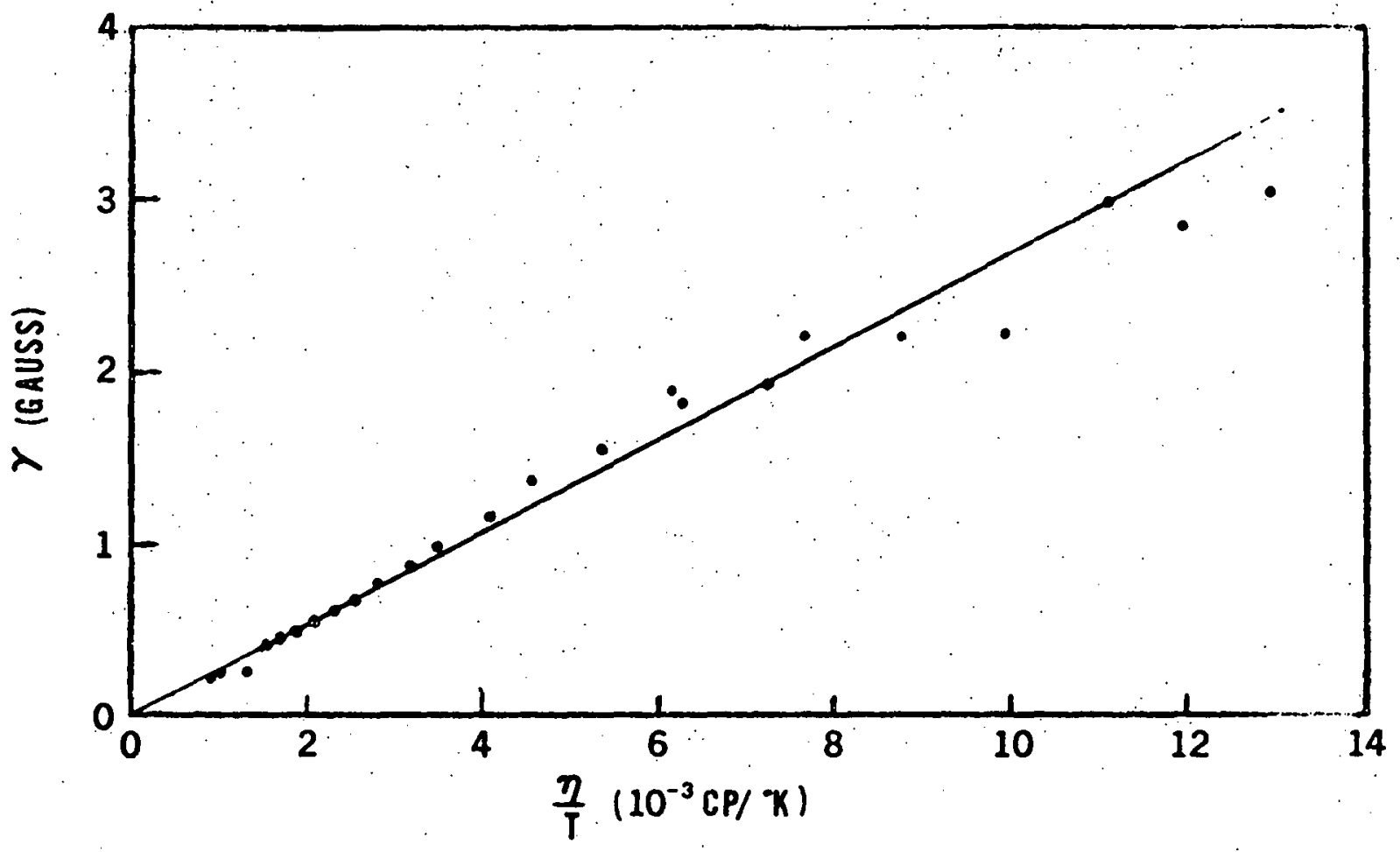

XBL 7012-7343

FIg. 8. Gamma parameter vs. $\mathrm{n} / \mathrm{T}$ for $\mathrm{Vo}\left(\mathrm{H}_{2} \mathrm{O}\right)_{5}^{2+}$. 


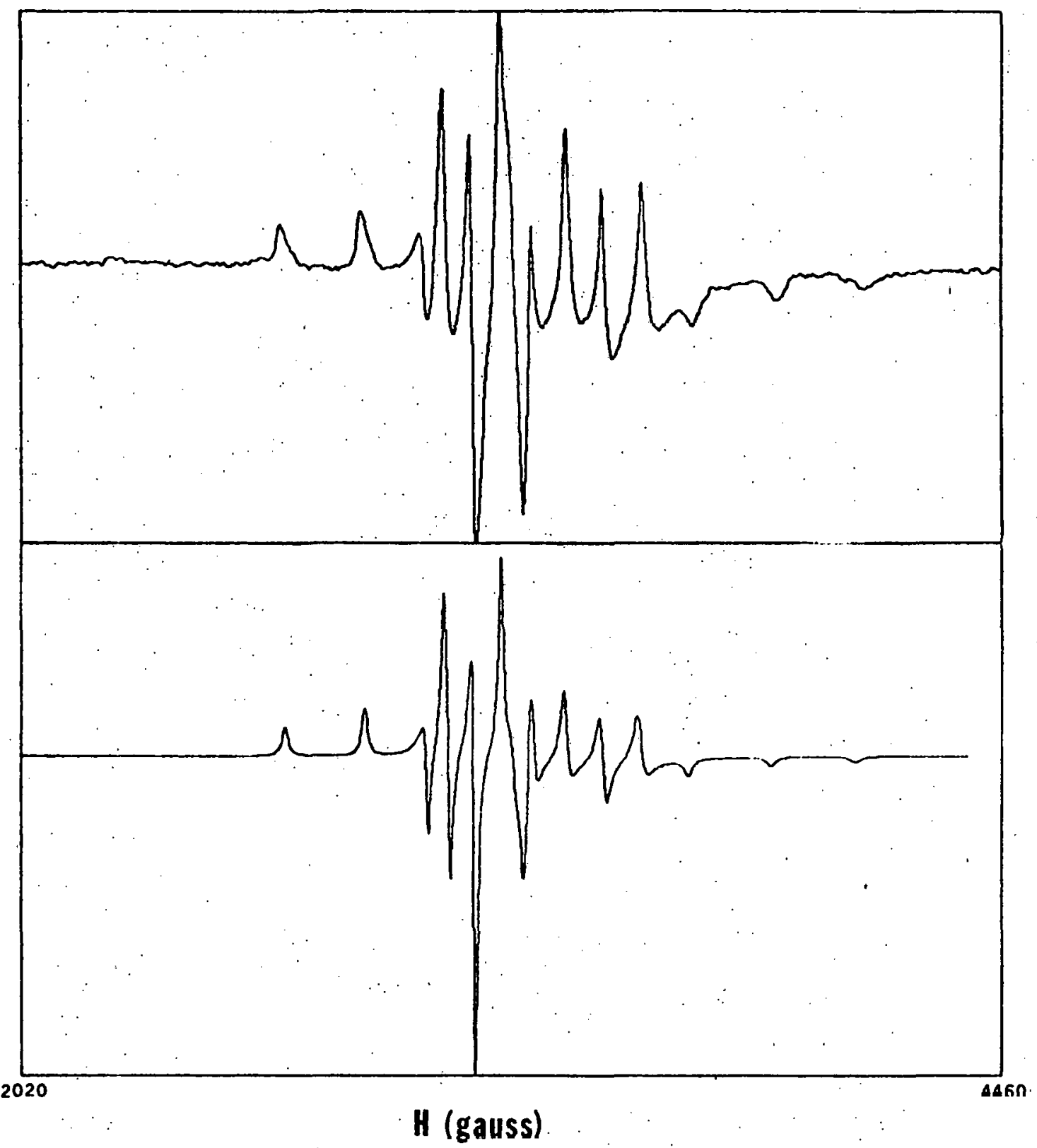

Fis. 9. $\operatorname{vo}\left(D_{2}\right)_{5}^{2+}$ glosi spectrum. Upper spectrum is experimental; lower spectrum is simulation. 


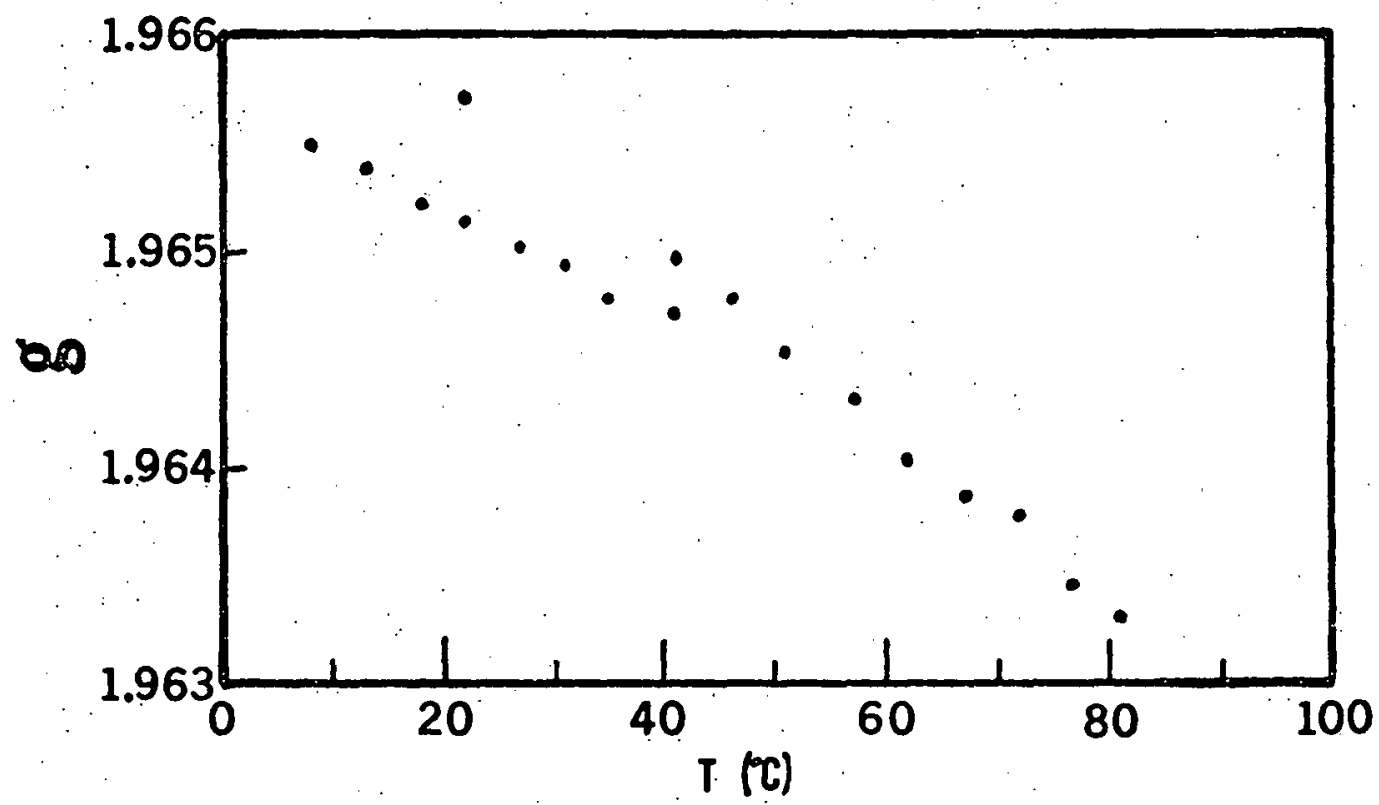

XBL $7012-7347$

Fig. 10. g vs. $T$ for $\operatorname{vo}\left(\mathrm{D}_{2} \mathrm{O}\right)_{5}^{2+}$. 
$-40-$

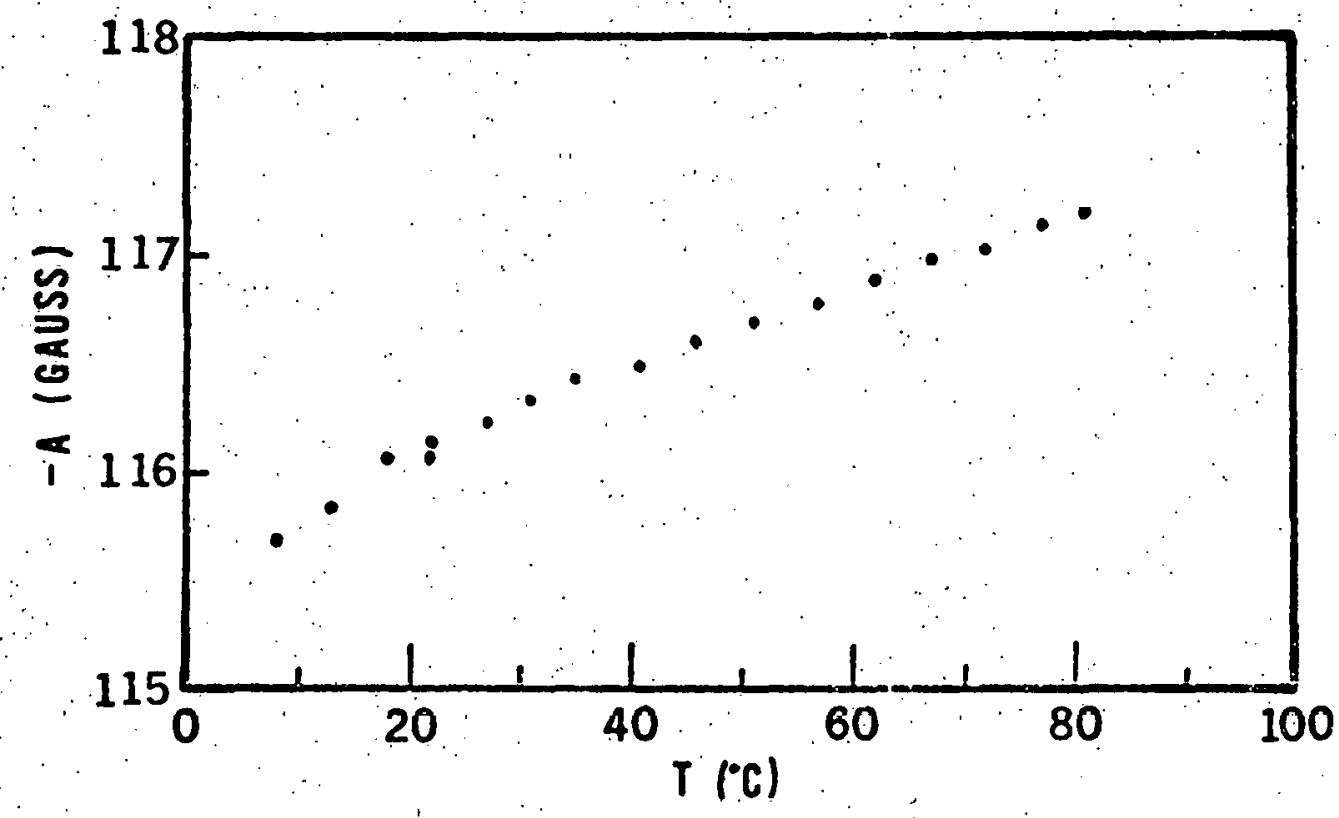

XBL $7012-7346$

Fig. il. -A vs.T for $\operatorname{Vo}\left(\mathrm{D}_{2} \mathrm{O}\right)_{5}^{2+}$ 


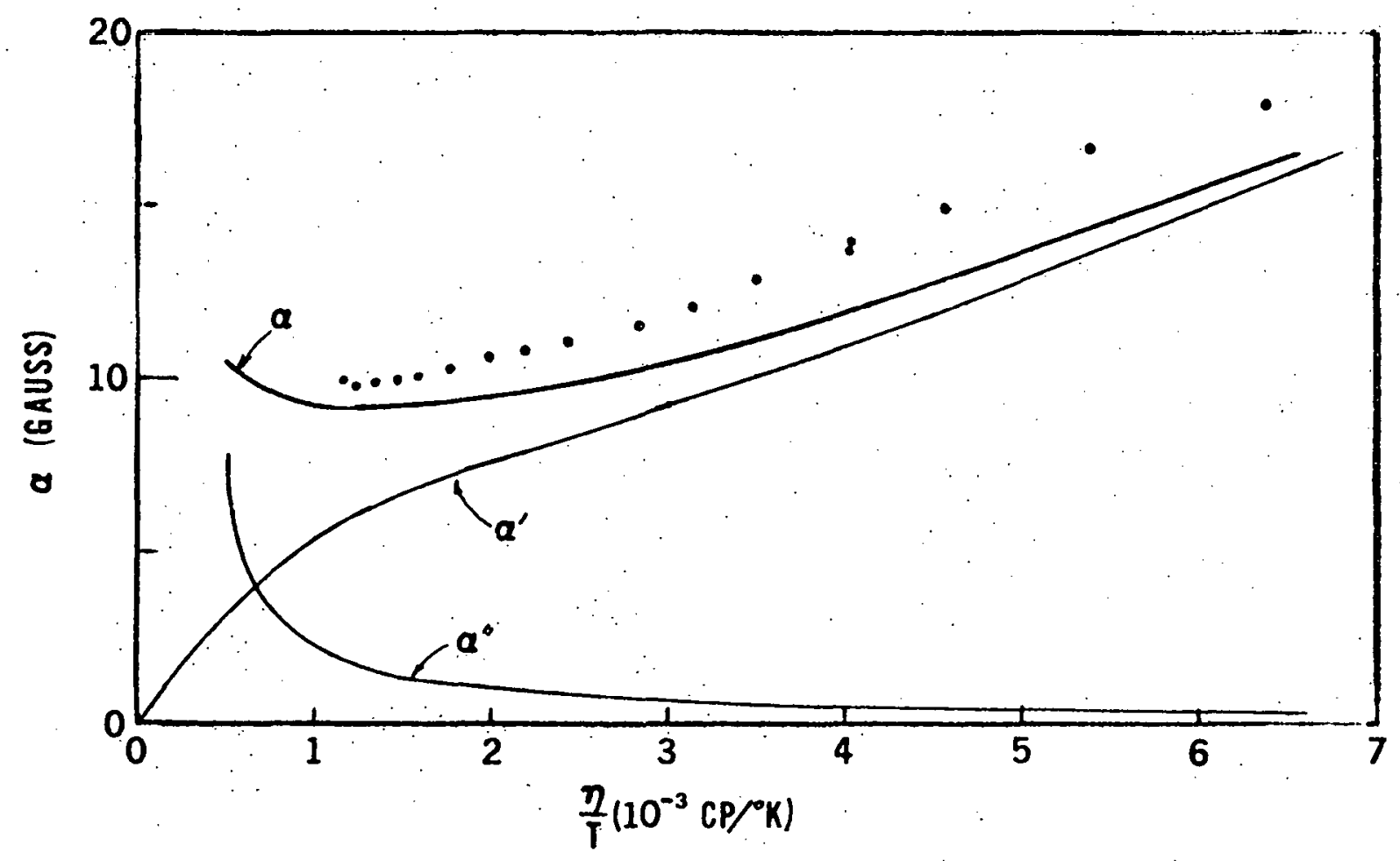

XBL $7012-7344$

Fig. 12. Alpha parameter vs: $n / T$ for $V o\left(D_{2} O\right)_{5}^{2+}$. 
$-42-$

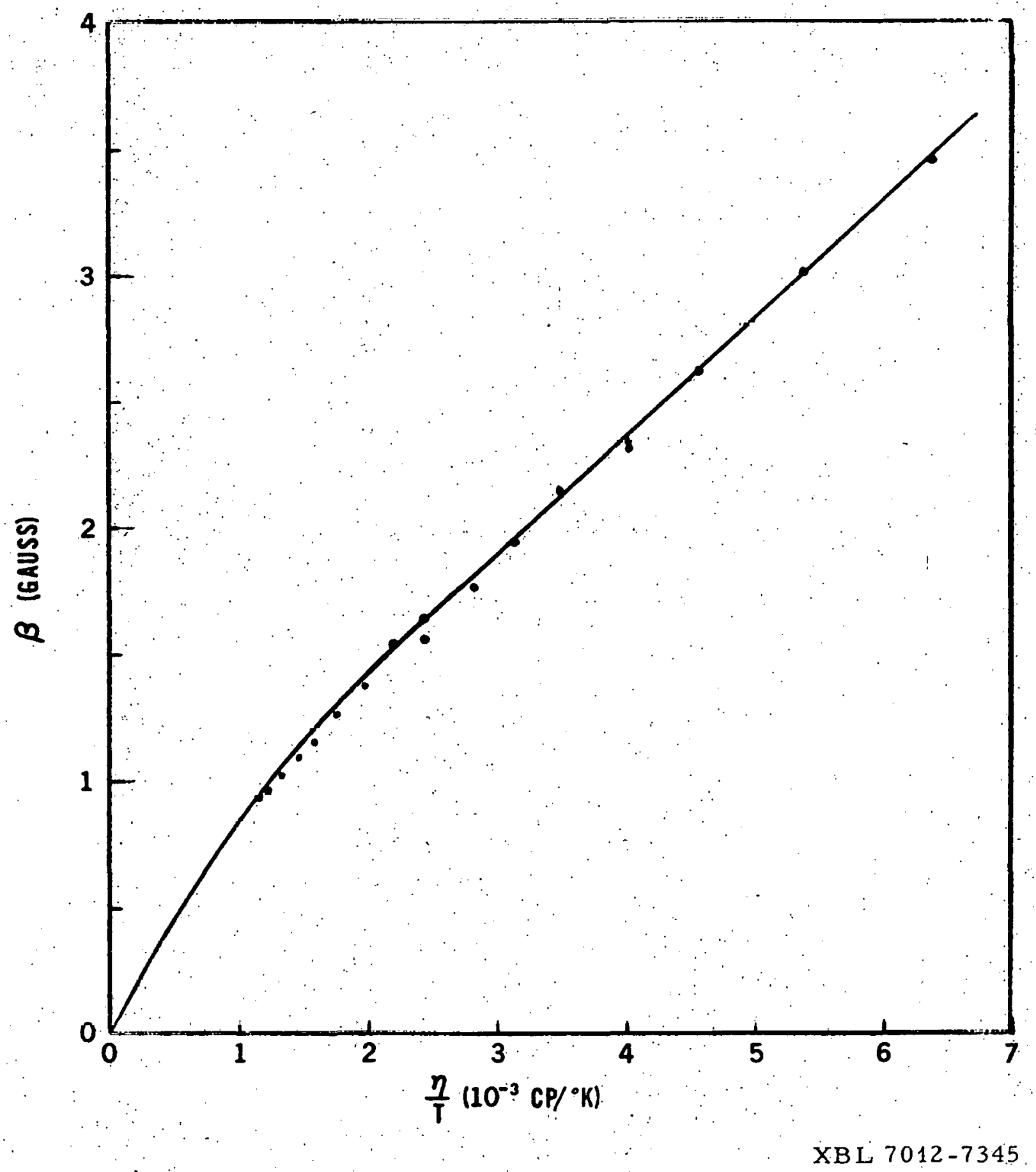

Fig. 13. Beta parameter vs. $n / T$ for $\operatorname{vo}\left(D_{2} O\right)_{5}^{2+}$. 


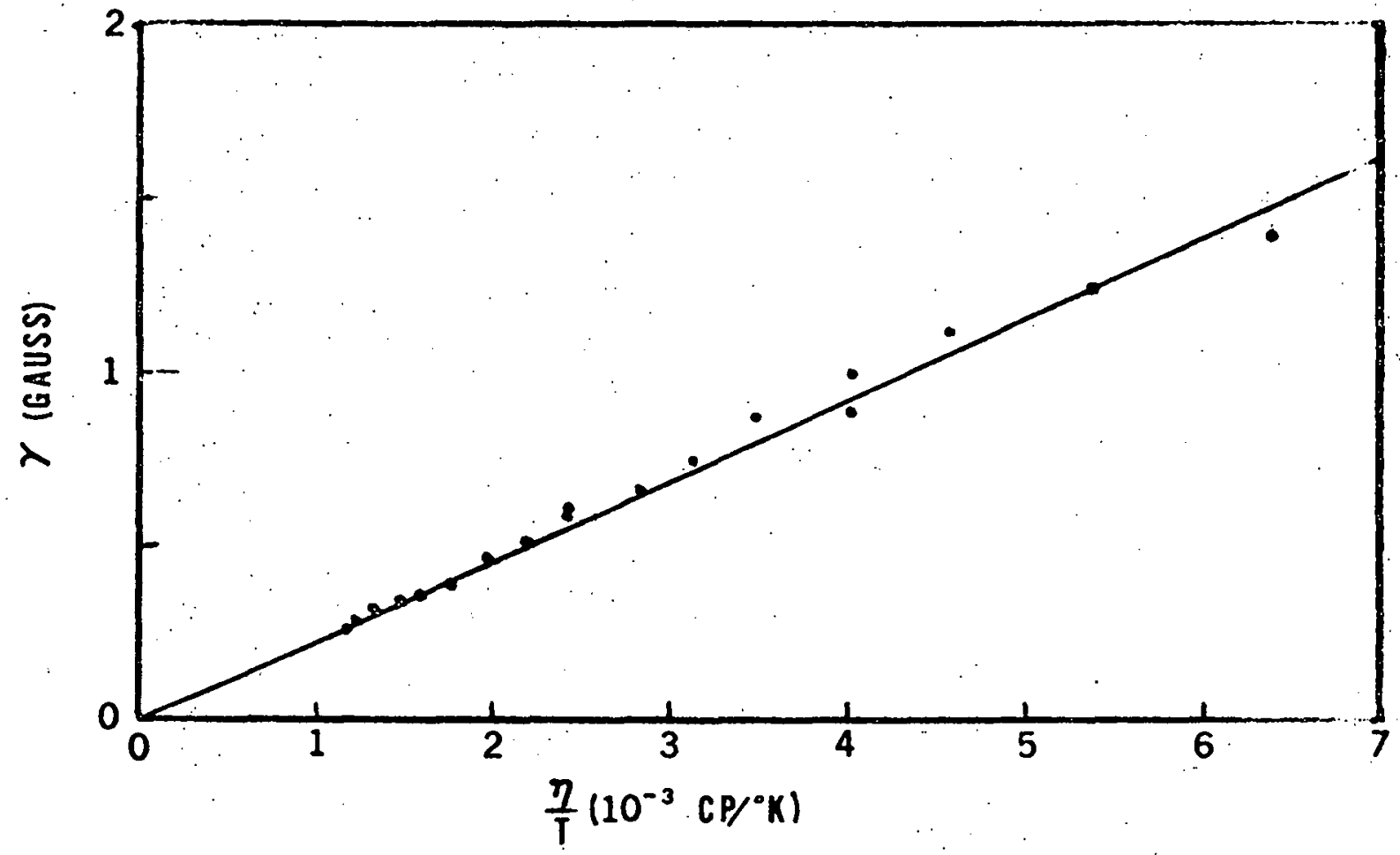

XBL $7012-7350$

Fig. 14. Gamma parameter vs. $n / T$ for $v o\left(D_{2} 0\right)_{5}^{2+}$. 
TABLE II. Viscosities of $\mathrm{H}_{2} \mathrm{O}$ and $\mathrm{D}_{2} \mathrm{O}$

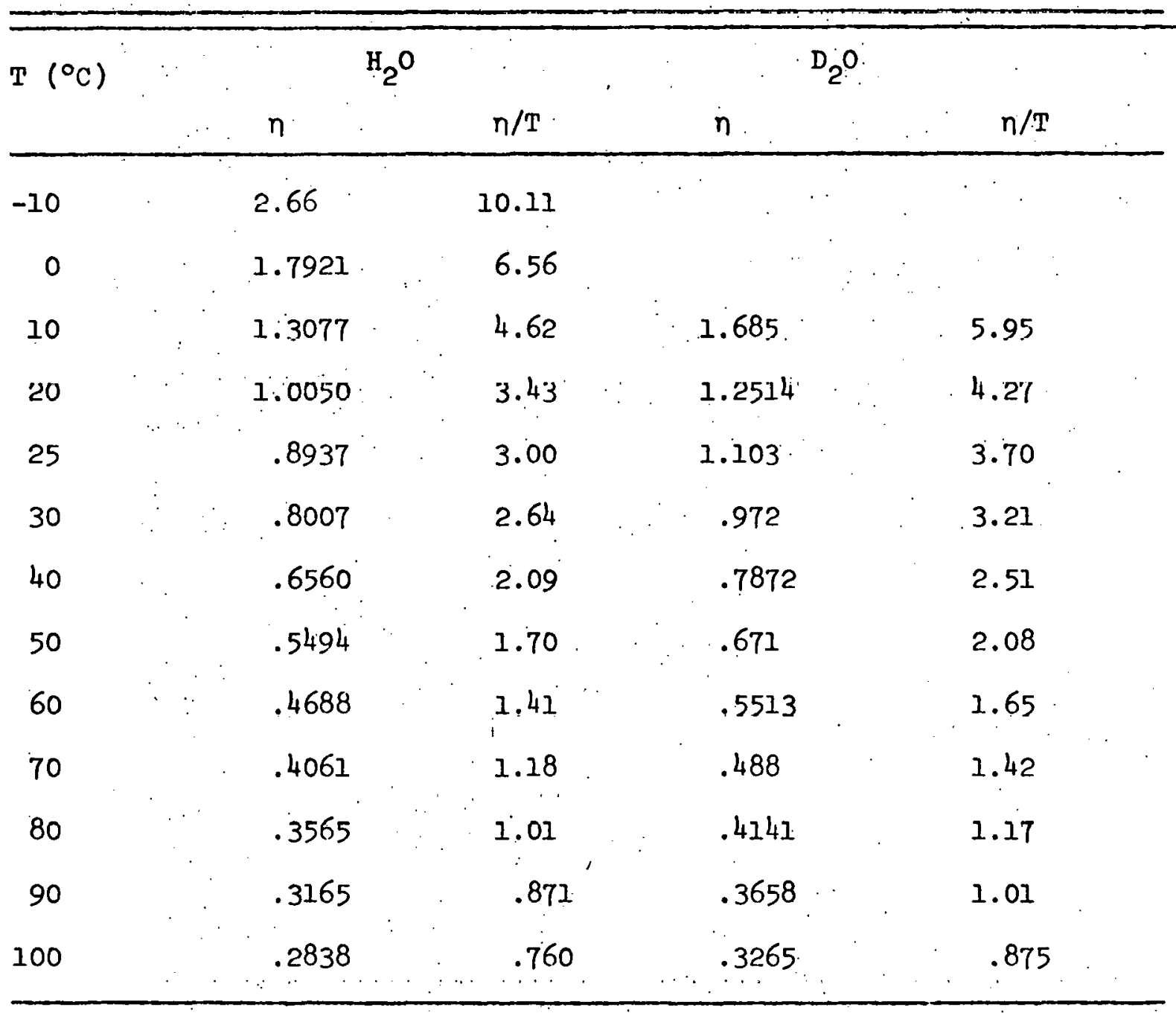

Note: Viscosities are in centipoises. Viscosities divided by temperature are in $10^{-3} \mathrm{cP} /{ }^{\circ} \mathrm{K}$.

Viscosities for $\mathrm{H}_{2} \mathrm{O}$ are from the Handbook of Chemistry and Physics, 43rd edition, CHemical Rubber Publishing Co.

Viscosities for $D_{2} O$ are from R. C. Hardy and R. L. Cottington, J. Chem. Fhys. 17, 509 (1949), and rrom the Landul-Burnstein Tubles, Vul. 5, Transport phenomena, Springer-Verlag (1969). 
copper linewidths. The variation of these parameters with temperiture forms a limitation on the interpretation of the linewidths. However, the variation is relatively small and does not seriously affect the theory. The values assumed for the linewidth treatment are $g=1.9652$ and $A-115.9 G$. The values used by. McCain (1967) were $g=1.9623$ and $A=-119.5 G$. The values predicted from the anisotropic parameters ( $g=1.9627$ and $A=-117.62(x)$ are slightly different, but the above values are thought to be better.

\section{Relaxation}

The vanadyl linewidths were least squares fit to a polynomial cubic in. $m_{I}$ in order to determine experimental values for the coefficients in Eq. 2.4. These values are plotted vs. viscosity divided by temperature as the points in Figs. 6-8. Selected values of the viscosity of water are presented in Table II to aid in the interpretation of the scales. The beta parameter was used to determine a value for the hydrodynamic radius of the complex from Eqs, 2.6 and 2.3. The radius, $r=3.67 \mathrm{~A}$, determined in this manner was used with Eqs. 2.5 and 2.7 to compute theoretical values for $\alpha^{\prime}$ and $\gamma$. The value for $\alpha^{\prime \prime}$ was computed using Eq. 2.12. The theoretical values are shown as the smooth curves in Figs. $6-8$.

The theoretical and experimental values of the gamma parameter agree very well. This provides strong support for the Kivelson relaxation mechanism. Furthermore, the hydrodynamic radius determined from the theory is reasonable compared with the radius one would obtain from only structural considerations. The delta parameter is in relatively poor agrecment with cxperiment, but terms of order $\left(a / \omega_{0}\right)^{2}$ have been neglected in the theory which contribute appreciably to the delta term 
(Wilson and Kivelson, 1966a).

However, al.though the theoretical alpha parameter $\left(\alpha=\alpha^{\prime}+\alpha^{\prime \prime}\right)$ is in good qualitative agreement with the experimental values, the magnitudes are not in good agreement. Lewis and Morgan (1968) proposed that this discrepancy could be due to superhyperfine interaction with the protons of the complexed water molecules. To check this proposal ve have made measurements of vanadyl ions dissolved in heavy water.

The anisotropic parameters for this system were determined from a deuterated perchloric acid glass. As expected. (since the vanadium crystal field is determined mainly by the strongly bonded vanadyl oxygen), the parameters were the same as those for the aqueous system (in this section only, the term "aqueous" refers to ordinary water solutions as opposed to heavy water). The polycrystalline spectrum is shown in Fig. 9. The theoretical curve in Fig. 9 is the same as that in Fig. 2. The isotropic $g$ and $A$ values for the deuterated vanadyl system are shown in Figs. 10 and 11 . The magnetic parameters used in the linewidth treatment were the same as those in the aqueous system.

The linewidth parameters are shown in Figs.12-14. As in the aqueous system, the beta parameter was used to determine a hydrodynamic radius from the theory. It is interesting that the radius, $r=3.48 \mathrm{~A}$, for the deuterated system is $0.19 \mathrm{~A}$ smaller than the radius in the aqueous system. This may indicate an actual change in the effective size of the complex, or more likely, a change in the value of $k$ (see Eq. 2.14) for the deuterium oxide solvent from the hydrogen oxide snivent.

The agreement between the theoretical and experimental values for the gumma term is excellent. However, the alpha term shows a discrepancy 
similar to that in the aqueous system. If the residual Iinewidth is due to a superhyperfine interaction, it would decrease by close to a factor of three since the magnetic moment of the deuteron is that much smaller than that of the proton. A comparison of the two residual widths indicates that the deuterated complex does not have a smaller discrepancy.

Recently, however, the proton magnetic resonance work was reported for the vanadyl system (Reuben and Fiat, 1969a, b). These workers give a proton superhyperfine coupling constant of $3.2 \mathrm{MHz}$, or $1.2 \mathrm{G}$ for $g=1.97$. Although this is sufficiently. large to provide the residual linewidth in the aqueous vanadyl system, it does not explain the behavior of the deuterated complex.

It must be noted that the samples were not degassed so that the residual linewidth could be due to oxygen broadening. However, McCain (1967) indicates that the vanadyl widths did not narrow upon degassing. A preliminary investigation by the author also produced this result. The effect of dissolved oxygen is, therefore, expected to be quite small.

We are then led to somewhat of a dilemma for the vanadyl system. Since the residual width did not change upon deuteration, this width cannot be due to superhyperfine structure. However, if the residual width is ascribed to other causes, we must show why no hyperfine effect is shown. We must therefore reexamine the premises of the experiment.

A resonance line may be homogeneously or inhomogeneously broadened: A homogeneously broadened line is a single line which may be characterized by a "true" linewidth. An inhomogeneous line is a superposition of several homogeneous lines which have a "true" linewidth. The inhomogeneous line has an apparent width which is, in general, not the "true" vidth. 
However, as the "true" width becomes larger and larger the apparent width of an Inhomogeneously broaden line should be closer to the natural width. If the "true" width is very large compared to the separation of the components, the apparent width will become equal to the "true" width.

A proton hyperfine coupling constant of $1.2 \mathrm{G}$ would surely produce a superhyperfine pattern in the vanadyl lines since proton exchange is slow. This should caluoe the vanadyl lluss th he inhomogencously broadened so that least squares fit to determine the linewidth should produce an error in the linewidth. However, as the true linewidth becomes larger, the error in the linewldth is expected to become smaller. Since the linewidths in the vanadyl spectrum at low temperatures are auite large compared to the hyperfine splitting, the residual linewidth due to hyperfine structure should go to zero. We see from Figs. 6 and 12 that the residual width is either constant with temperature or increases as temperature is decreased. Hence, we musl cunllude that, becausc thc residual width neither decreased with deuteration nor decreased with decreasing temperature, the main contribution to the residual width is not a superhyperfine interaction.

We would expect some contribution to the width from superhyperfine interaction. The residual width should have changed some upon changing to the heavy water solvent. The effect, however, is reduced beususe of the variation of linewidth with hyperfine component. The error of residual width in each hyperfine component due to superhyperfine interaction changes from component to component. The error in the alpha parameter would be a kind of average over the errors for all the components. For some of the lines the wlaths are large enough that the error is close 
to zero. Hence, the average contribution to the residual width is much smaller than the maximum possible contribution. Furthermore, the linewidth variation is not symetric with respect to the center of the spectrum. The nerrowest line in the spectrum is to the low field side of the spectrum center. The result of this is that the beta and gamma parameters must also be affected by the superhyperfine interaction. The contribution of superhyperfine structure to. the alpha parameter is much smaller than the possible contribution to a given line. The change in the residual width upon going to a deuterated solvent is smaller than the residual width due to superhyperfine interaction. It is not observed because it is so small.

We, therefore, have an unexplained residual width in the vanadyl system. The residual width cannot be due to the Al'tshuler and Valiev (1958) mechanism since this mechanism is temperature dependent. Furthermore, this mechanism would predict a linewidth increasing as temperature increases. The residual width on the other hand is constant or decreases with increasing temperature.

The linewidth in the vanadyl system is, nevertheless, almost completely explained by a combination of the spin-rotation and anisotropic 8 and $A$. tensor mechanisms. We may test the field dependence of the mechanism by comparing theoretical predictions with the parameters measured by McCain (1967) and by Rogers and Pake (1960). These parameters are presented in Table III.

A comparison of the $S$ band experimental and theoretical values shows a considerable disagreement. The alpha term is off by 5 gauss; the beta and gama terms also disagree. McCain indicated that this disagreement 
TABIE III. Comparison of Theoretical and Experimental Linewidth Parameters for $\mathrm{VO}\left(\mathrm{H}_{2} \mathrm{O}\right)_{5}^{2+}$

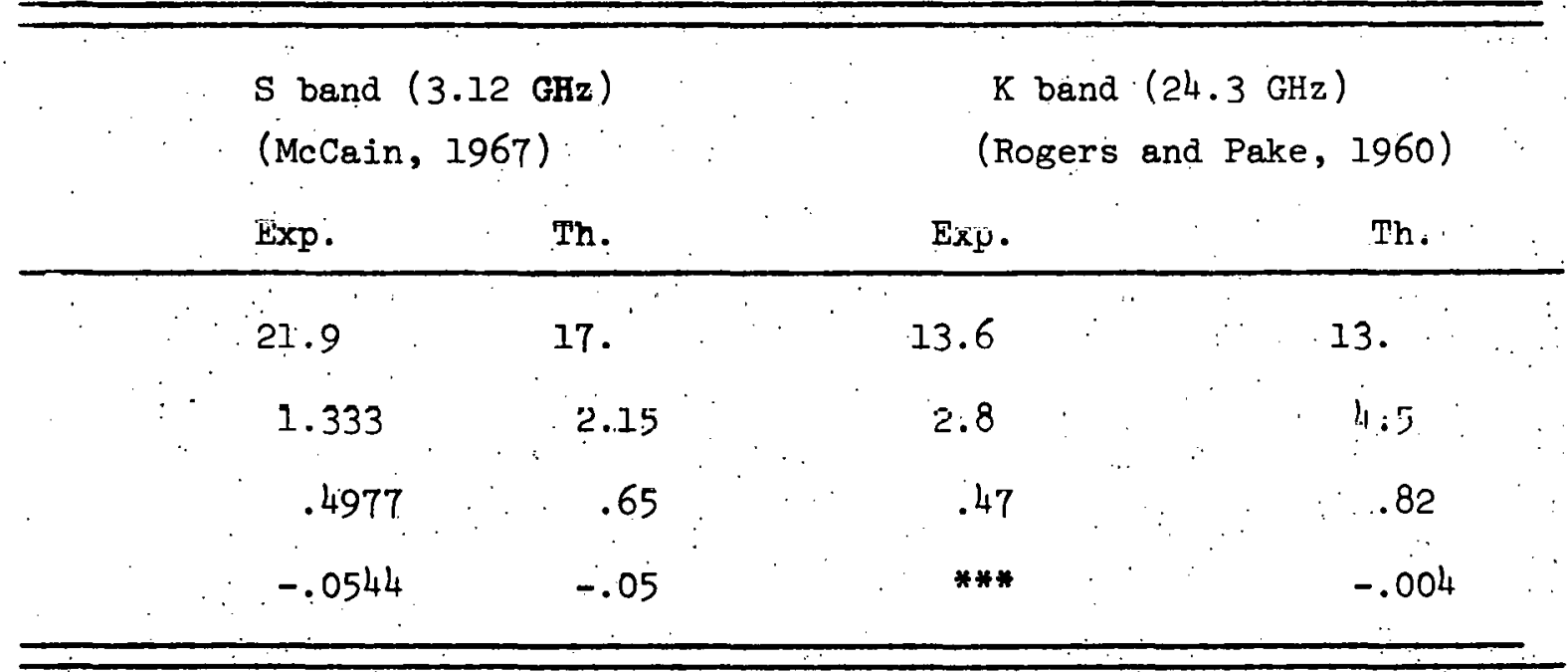


was probably due to terms of order $\left(a \omega_{0}\right)^{2}$ which were neglected in the theory. We feel that this is the most probable reason. Furthermore, this is probably also the reason for the residual width in the $\mathrm{X}$ band data. The neglected terms have relatively little effect on the beta and gamma parameters, but seriously affect the alpha parameter. This discrepancy is expected to be smaller at $K$ band where the neglected terms would be quite small. A comparison of the $K$ band experimental and theoretical data is somewhat disappointing. Although, the alpha term agrees well with theory, the beta and gamma terms are extremely wrong. We suspect that this is due to an error in the data rather than in the theory. Rogers and Pake indicate that the lines have some non-Lorentz character which we believe is an experimental problem which produced an error in the results. $K$ bands experiments are quite difficult because the sample must be small to avoid distortion problems. The Kivelson theory is then expected to completely explain the linewidths. It would be interesting to obtain some additional $\mathrm{K}$ band data.

The theory may be used to compute theoretical estimates of the relative $T_{1 e}$ values for comparison with McCain's (1967) experimental values. However, the theory is only approximately correct for the $S$ band data. McCain used a correlation time of $\tau=3 \times 10^{-11} \mathrm{sec}$ in his work where the value determined in this work is $\tau=3.8 \times 10^{-11} \mathrm{sec}$. However, this change does not. change the results enough. The agreement between theory and experiment is aute poor, but this is expected since the linewidth predictions are also poor. 
IV. COPPER LINEWIDTHS

\section{A. Introduction}

The EPR spectrum of the hexaquocopper (II) complex in solution was certainly one of the first to be studied though it is difficult to say when it was first observed. Among the earliest studies of the system were those of Kozyrev (1955) and McGarvey (1956, 1957). These workers reported a spectrum consisting of a single line at ordinary temperatures with a $\mathrm{g}$ value of about 2.2 and a peak-to-peak linewidth of about 150 gauss. Although the copper nucleus has a nuclear spin $I=3 / 2$, no hyperfine structure was observed in the spectrum.

The intriguing characteristic of the spectrum was that the line width was observed to increase with increasing temperature (Kozyrev, 1957; Avvakumov, et al., 1960). At first glance the linewidth of this spin $1 / 2$ complex would have been expected to decrease with increasing temperature on the basis of the McConnell theory (1956).

A number of explanations were proposed for the anomalous behavior of the copper system. Indeed, Kozyrev (1955) early proposed that the broadening and subsequent lack of hyperfine structure in the copper spectrum was due to the formation of copper dimers. This suggestion was based on an incomplete understanding of some of the early data, and is not tenable since the linewidth behavior persists in dilute solutions where copper dimers are quite unlikely. McGarvey (1957) proposed that the linewlath was due to a combination of tumbling and an interaction with a low lying exclted state which was expected in the hexaquocopper (II) system as a result of the Jahn-Teller effect. Al'tshuler (Al'tshuler and Valiev, 1958; Al'tshuler and Kozyrev, 1964) proposed a theory involving vibrational. 
modulation of the crystal fleld. He showed that this sheory would fit their data auite weil.

However, all the early theories suffered from incomplete information as to the true linewidth in the system. The linewidth was taken as the derivative peak-to-peak distance of the broad single line observed in the spectrum. But the EPR spectrum of hexaquocopper (II) nust certainly consist of hyperfine structure. Hayes (1961) detected hyperfine structure in the spectrum by cooling to near $0^{\circ} \mathrm{C}$. The coupling constant between 31 to 38 gauss, indicated that the linewidth was in a large part due to hyperfine structure.

Among other workers who have used the overall linewidth are Valiev and Zaripov (1966) and Fufiwara and Hayashi (1965). Valiev and Zaripov proposed a relaxation mechanism specific, to the hexaquocopper (II) system. This mechanism, a modification of the Al'tshuler and Viliev (1958) mechanism, involved a vibrationally induced transition to the lowest orbital excited state with subsequent relaxation to the ground state. This is a kind of Orbach mechanism involving ligand vibrations rather than lattice modes. Valiev and Zaripov's work shows reasonable agreement with the overall linewidth if the hyperfine contributions are ignored.

Fujiwara and Hayashi studied the linewidth as a function of temperature, as a function of concentration, and as a function of the anion associated with the copper cation. These workers report no anion dependence in dilute solutions and also no concentration dependence for solutions less than .1 F. These results are qualitatively correct since although the true linewidth was not measured, a concentration effect would be observed in the overall I1ne. Although these workers report measurements 
near $0^{\circ} \mathrm{C}$, they report no hyperfine structure in the spectrum.

Spencer (1965) measured the EPR spectra of hexaquocopper (II) over a wide range of temperatures for both aqueous solutions and for perchloric acid solutions. He also measured the spectra of the hexamminecopper (II) system which was expected to be quite similar in behavior. Using the hvperfine coupling constant of $.0053 \mathrm{~cm}^{-1}$ he was able to perform crude corrections to the linewidths. At low temperatures he observed the Inewidth to decrease as the temperature increased. Spencer discusses two mechanisms to explain the linewidth: the anisotropic tumbling mechanism. and an inversion mechanims. Although a definite conclusion was not reached, Spencer proposed that the Inewldth could be explained by a combination of these mechanisms. Another explanation was that the linewidth was controlled by the rate of chemical exchang of the water molecules in the hydration sphere. Additional support for this proposal was obtained from the ${ }^{17} 0 \mathrm{MMR}$ data (Meredith, 1965). The ${ }^{17_{0}}$ Iinewidth was observed to vary with the same temperature dependence as the FPR linewidth.

Lewis, Alei, and Morgan (1966) also studied the copper system as a function of temperature. They study the copper system by means of a lineshape analysis using a simulation technique. At high temperatures (above room temperature) they assume that all the linewidths are equal. From their analysis they conclude that the linewidth should be due to a combination of the spin-rotational process and a second process which they describe as a Raman process. They propose the Raman process on the basis of extrapolation from reported solid state measurements. However, Kivelson (1966) indicates that Raman processes are not expected to be large in solution. Lewis, Alei and Morgan propose the following 
function for the linewidth

$$
\mathrm{T}_{2}^{-1}=2.04 \times 10^{4} \cdot\left[(\mathrm{T} / \mathrm{n})+0.23 \mathrm{~T}^{2}\right]
$$

The main difficulty with the hexaquocopper (II) system, other than the actual interpretation of the linewidth behavior, is the measurement of the true linewidths for the hyperfine lines. The large linewidths leading to extreme overlap and lack of resolution in the spectrum produces an extremely frustrating problem. Simulation methods of analysis for lines as unresolved as the copper lines are very difficult. The present study was begun because it was felt that the use of a data acquisition system in connection with a least squares treatment would facilitate the treatment of the data and improve the results.

\section{B. Experimental Methods}

The apparatus and measurement procedures were the same as those described for the vanadyl experiments (see Sec. III-B). The initial experiments were performed with copper perchlorate obtained from the G. Fredertck Smith Company: The final experiments were performed with isotopically enriched ${ }^{63} \mathrm{Cu}(99.62 \%)$ which was obtained from Oak Ridge National Laboratories in the form of the oxide. $\because$ The oxide was dissolved in an equivalent amount of perchloric acid with gentle heating. The concentration of the resulting copper perchlorate solution was adjusted to $.01-.02 \mathrm{~F}$. Solutions in $5.26 \mathrm{~F}$ perchloric acid for glass spectra were obtained by evaporating the necessary amount of the copper perchlorate aqueous solutions and then redissolving the residue in perchloric acid. 


\section{Discussion and Results}

\section{Spectra}

The anisotropic magnetic parameters for the hexaquocopper (II) system have proven to be quite elusive. A number of workers have determined these parameters in different glass systems, but have obtained varying results. These results are presented in Table III. In addition, we have repeated the measurements in the perchloric acid glass system.

Spencer (1965) originally measured the parameters for the perchloric acid glass system by measuring the line positions. The spectrum is quite simple and consists of a parallel band containing four resolved hyperfine components and a perpendicular band containing a doublet. The interpretation of this doublet is difficult without a simulation method to analyze the spectrum. We have redetermined the anisotropic magnetic parameters by using a simulation technique to analyze the spectrum.

Spencer suggests that the doublet could arise from either hyperfine structure or from two $g$ values due to the complex having less than tetragonal symetry in the glass, or from so-called "extra" absorptions. Hyperfine structure would produce four components and hence was expected to give four lines rather than two. However, the theoretical simulation in Fig. 15 shows that the spectrum can be fit quite well with an axial spin Hamiltonian. Only a doublet is observed because of a second order hyperfine effect which shifts two lines of the quartet further than the others. A variable linewidth further accentuates a doublet appearance over a quartet. The parameters determined from the analysis are presented in Table IV.

Table IV shows a large spread in the results of the various workers. , Of the three systems utilized, the methanol results are probably the least correct for the hexaquo complex. These measurements were performed with 


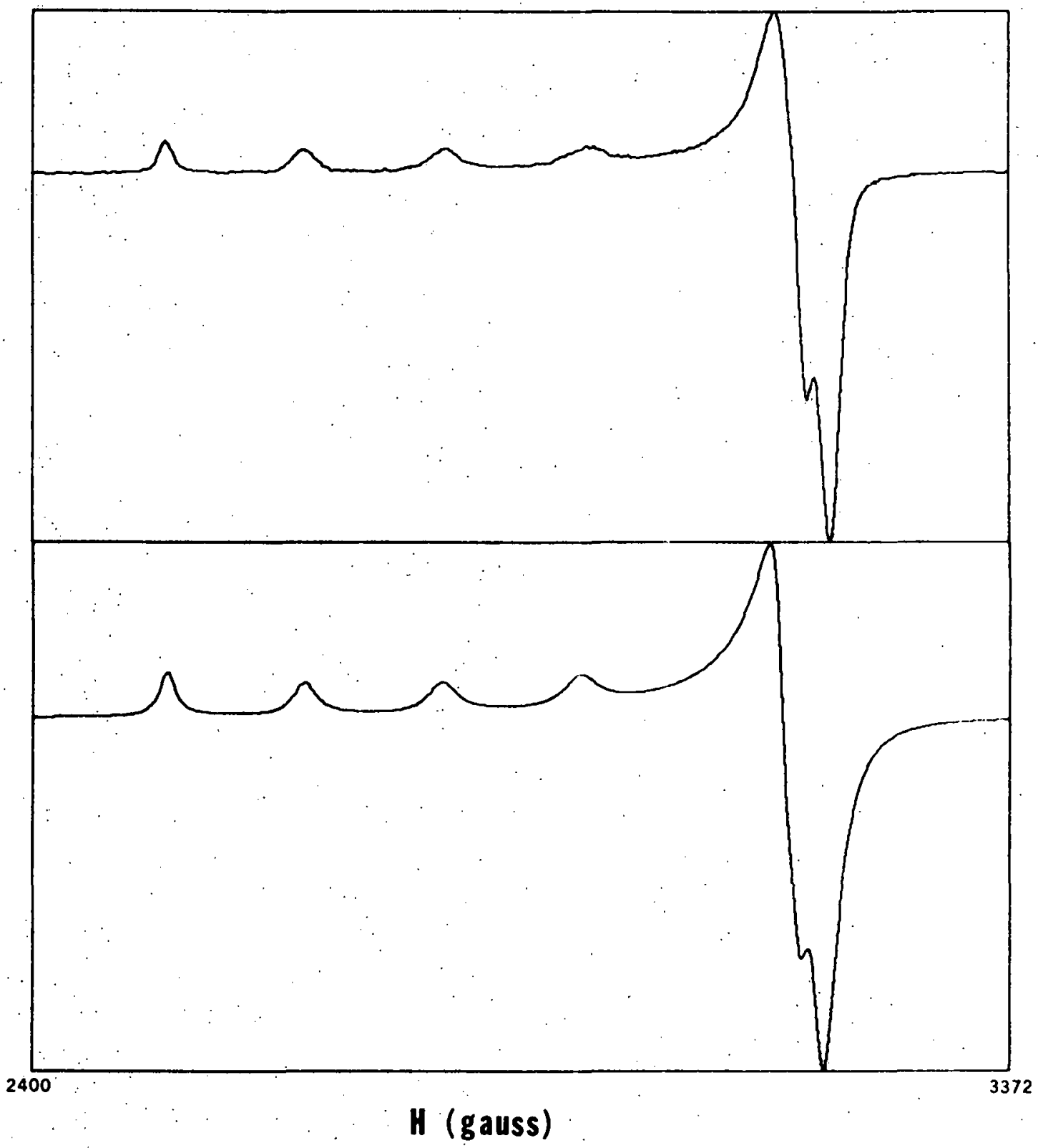

XBL $7.012-7175$

Fig. 15. Spectrum of $\mathrm{Cu}\left(\mathrm{H}_{2} \mathrm{O}\right)_{6}^{2+}$ in perchloric acid glass. Upper spectrum is experimental; lower spectrum is simulation. 
TABLE IV. Anisotropic Magnetic Parameters for $\mathrm{Cu}\left(\mathrm{H}_{2} \mathrm{O}\right)_{6}^{2+}$

\begin{tabular}{|c|c|c|c|c|c|c|c|}
\hline Solute/Solvent & $\varepsilon_{\|}$ & $3_{1}$ & $A_{11}^{a}$ & $A_{1}^{\varepsilon}$. & 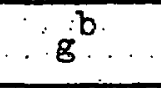 & $A^{8 ; b}$ & Reference \\
\hline $\mathrm{Cu}\left(\mathrm{NO}_{3}\right)_{2} /$ metharol & 2.39 & 2.07 & 106 & 70 & 2.1767 & 83.2 & Garif'yanov and Usacheva (1964) \\
\hline $\mathrm{Cu}\left(\mathrm{ClO}_{4}\right)_{2} / 5.3 \mathrm{FClO}_{4}$ & 2.379 & 2.065 & 139.6 & $i$ & 2.1703 & 46.5 & Spencer (1965) \\
\hline $\mathrm{Cu}\left(\mathrm{NO}_{3}\right)_{2} /$ glycerol & 2. LoO & 2.099 & 114.05 & 12.86 & 2.1993 & 33.3 & Lewis, et al. (1966) \\
\hline${ }^{63} \mathrm{Cu}\left(\mathrm{ClO}_{4}\right){ }_{2} / 5.3 \mathrm{HClO}_{4}$ & 2.387 & 2.072 & 137 & 5 & 2.177 & 46.9 & This work \\
\hline
\end{tabular}

a. Couplizg constants are in gauss

b. Average values computed from anisotropic values 
the hydrated salt in almost pure methanol. The results are probably incorrect due to the formation of copper-methanol complexes.

The results obtained for the $5.26 \mathrm{~F}$ perchloric acid and the $60 \%$ glycerol glasses are somewhat harder to reconcile. Initially, the perchloric acid system is expected to provide a better glass medium for the hexaquo complex since it is generally accepted that the perchlorate anion does not form complexes with transition metals although the same is not true for glycerol. Furthermore, there are more water molecules (40 F) available in $5.26 \mathrm{~F}$ perchloric acid that in $7.5 \mathrm{~F}$ glycerol (25 F in water).

However, the anisotropic parameters may be used to compute average g. and $A$ values which are expected to be close to the isotropic $g$ and $A$ values. The computed average values are also presented in Table IV. A comparison of these values with the experimental isotropic values which are presented later shows that the glycerol parameters agree quite well. But the perchloric acid parameters are very different from the experimental values." As mentioned in the vanadyl discussion, glass spectra need not necessarily produce correct values for the complex in solution. However, the measured difference is striking.

We may note that the $g$ and $A$ values are expected to be correlated due to bonding effects (Kivelson and Neiman, 1961). As the 8 value decreases; the A value should increase. This correlation seems to hold for the parallel parameters between the glycerol and the perchloric acid glass. However, the correlation breaks down in the perpendicular parameters. This might be a possible indication that different complexes are being observed. If both spectra were due to copper ions with octahedrally coordinated water 
molecules, the correlation would be expected to hold. "The choice of which set of parameters to use is somewhat arbitrary. Fortunately, the parameters desired for a linewlath theory are the anisotropies, i.e.; the differences between the parallel and perpendiuclar values, rather than the actual parameters. The anisotropies are in somewhat better agreement than the actual parameters. In fact, both sets of parameters were used for the Inewidth study.

The EPR spectra for hexaquocopper (II) ions in solution have been messured between -15 and $100^{\circ} \mathrm{C}$. The spectral parameters were obtained from the digitized spectra using the least squares fitting procedure described previously. The spectra could be fit very well using one intensity, one $g$ value, one coupling constant, and four linewidths. Examples of the fits to the spectra at various temperatures are shown in Figs. 16-19. The crosses are the experimental points; the continuous Iines are the theoretical fit. The lower curves in the figures are plots of the difference between the theoretical and experimental curves. We can see from the error curves that the fits are very good. The low temperature spectra (Figs. 16 and I7) exhibit the presence of four hyperfine lines quite clearly. However, the spectra at higher temperatures (Fig. 19) do not exhibit structure.

At room temperature and above the spectra consist of a single; broad, symetric line. Much of the early work has considered the peakto-peak whdth of the unresolved line to be the true wldth of the Iine. This would be true if the hyperfine coupling constant were small compared to the linewidth. The observed line would then be very nearly a Lorentz lineshape whose peak-to-peak width would be an excellent 


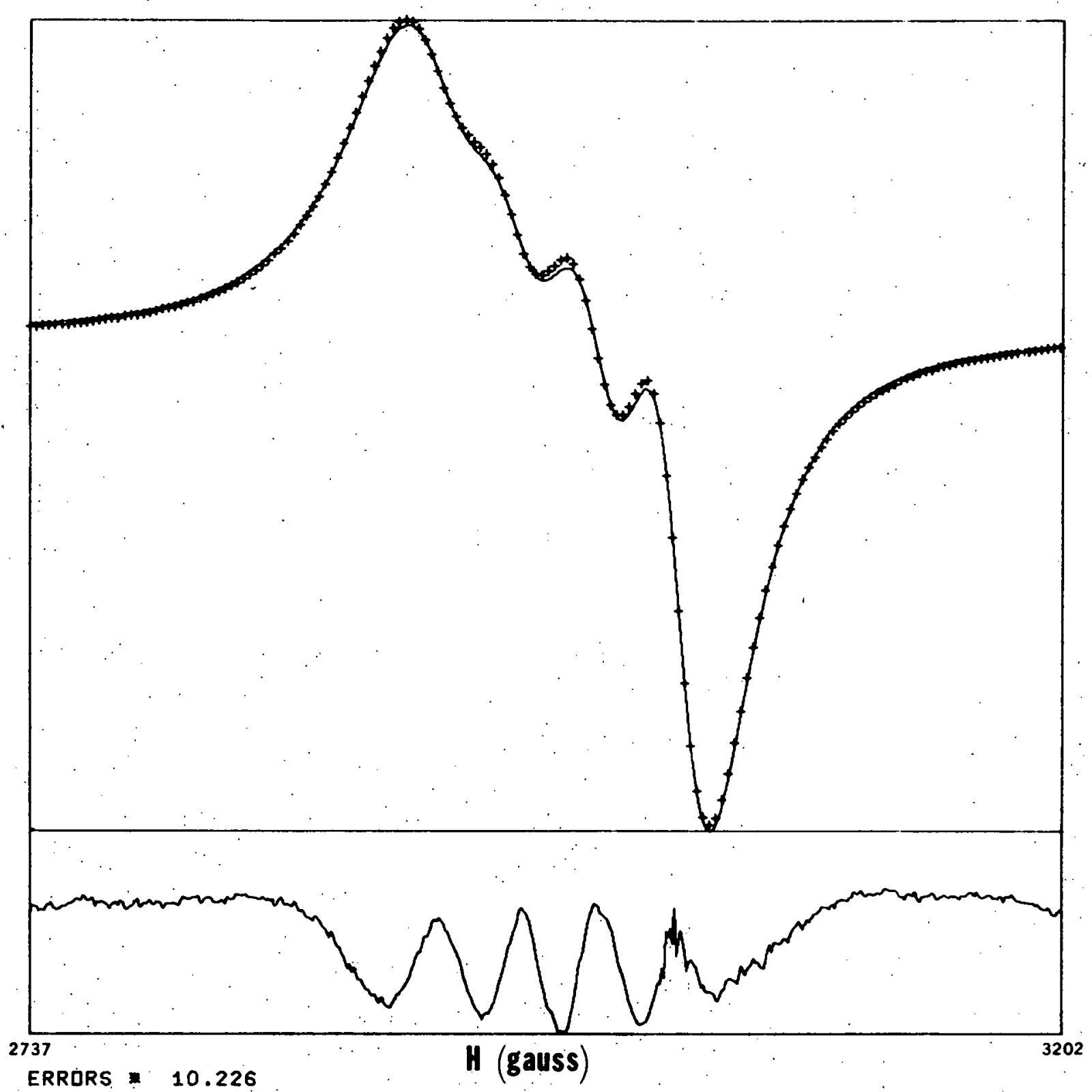

XBL $7012-7169$

Fig. 16. Example of fit to solution spectrum of $\mathrm{Cu}\left(\mathrm{H}_{2} \mathrm{O}\right)_{6}^{2+}$ at $-10^{\circ} \mathrm{C}$. In this spectrum and in the following spectra the crosses are the experimental points; the continuous Iine is the theoretical fit to the data; and the lower curve is the difference between the theoretical and experimental spectra with a scale expansion given hy the ERROR* number. 
$-62-$

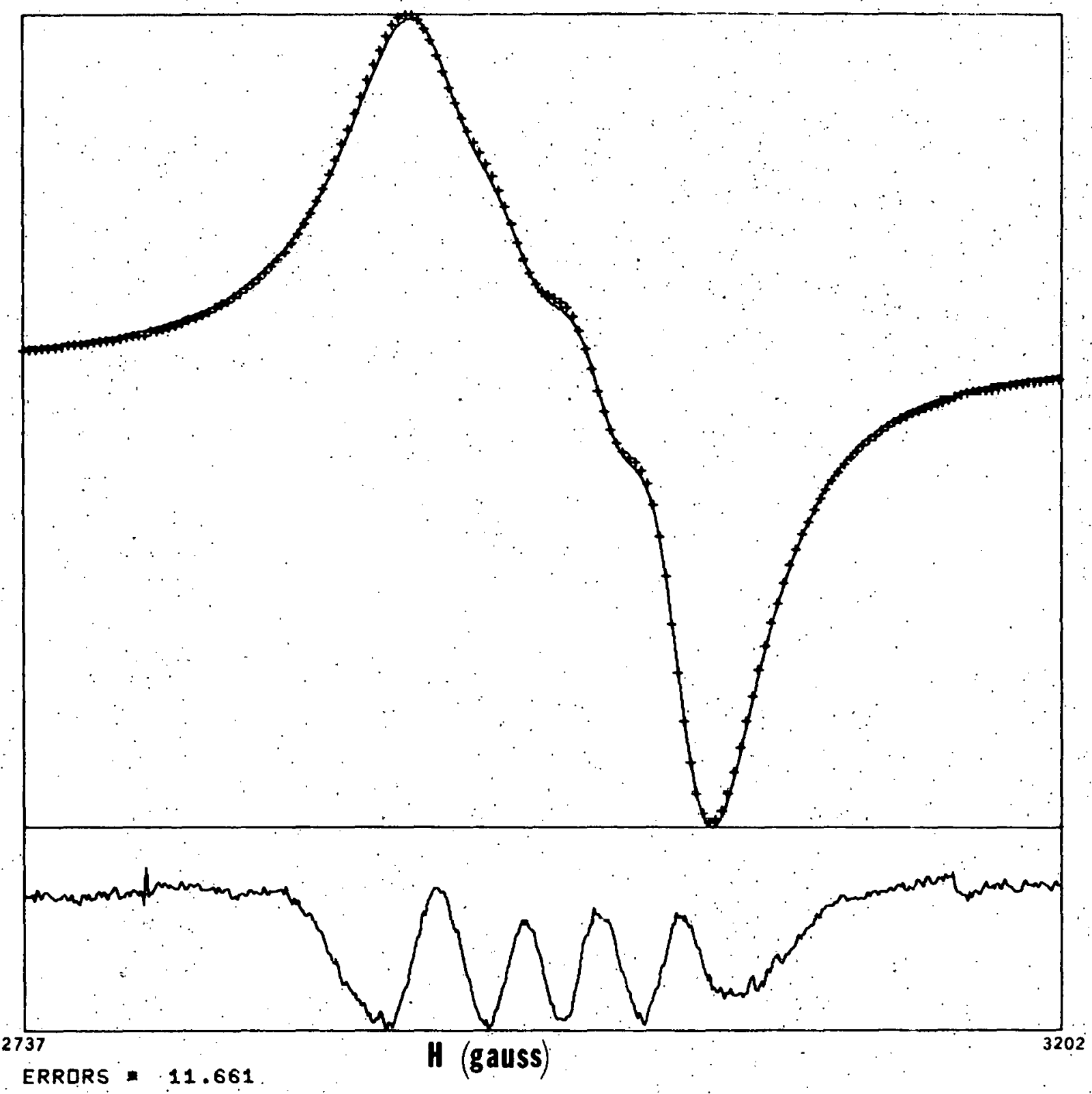

$$
X B L \div 012-7170
$$

Fig. 17. Example of fit to solution spectrum of $\mathrm{Cu}\left(\mathrm{H}_{2} \mathrm{O}\right)_{6}^{2+}$ at $1^{\circ} \mathrm{C}$. 
$-63-$

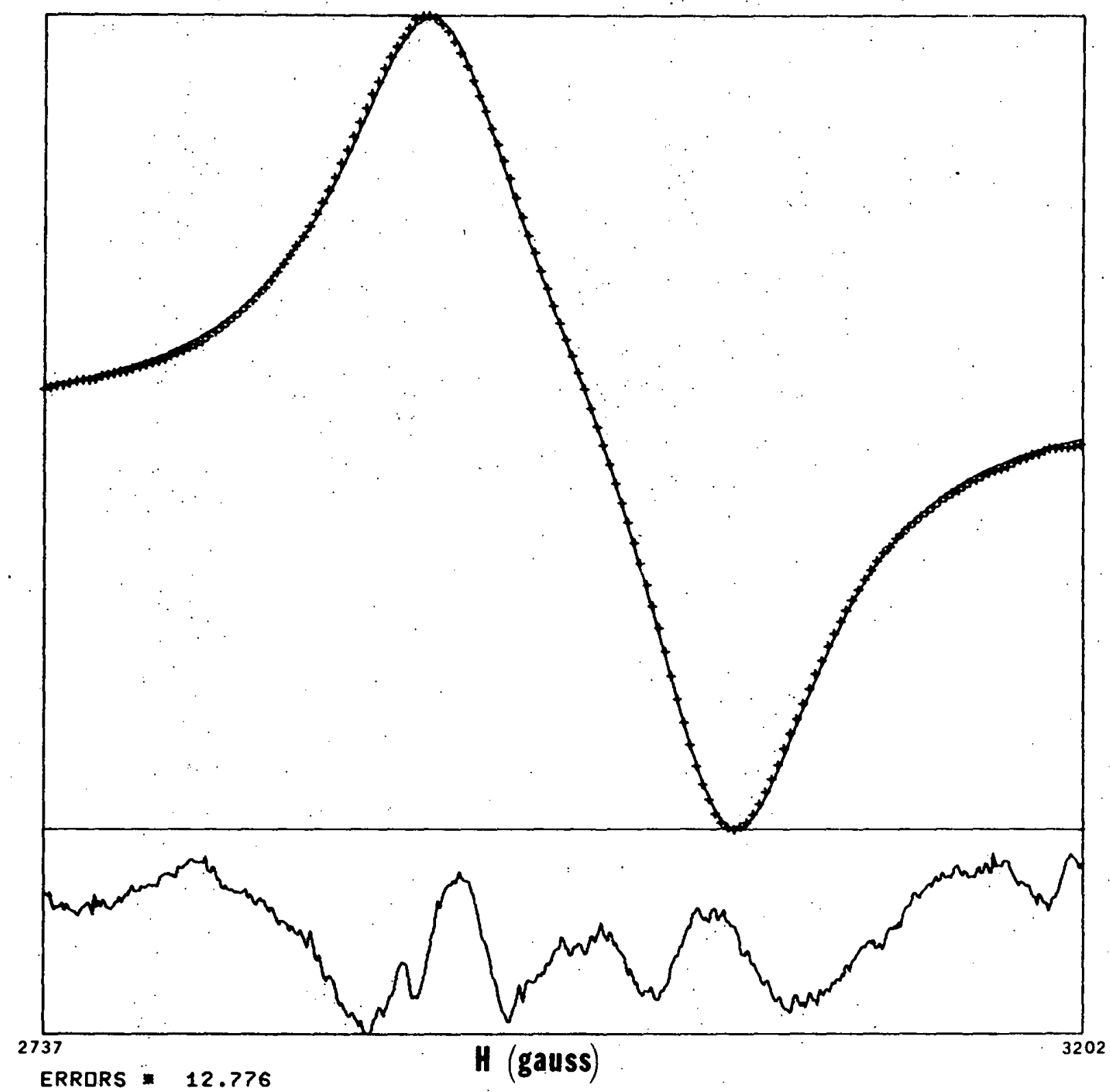

X BL $7012-7171$

Fig. 18. Example of fit to solution spectrum of $\mathrm{Cu}\left(\mathrm{H}_{2} \mathrm{O}\right)_{6}^{2+}$ at $22^{\circ} \mathrm{C}$. 


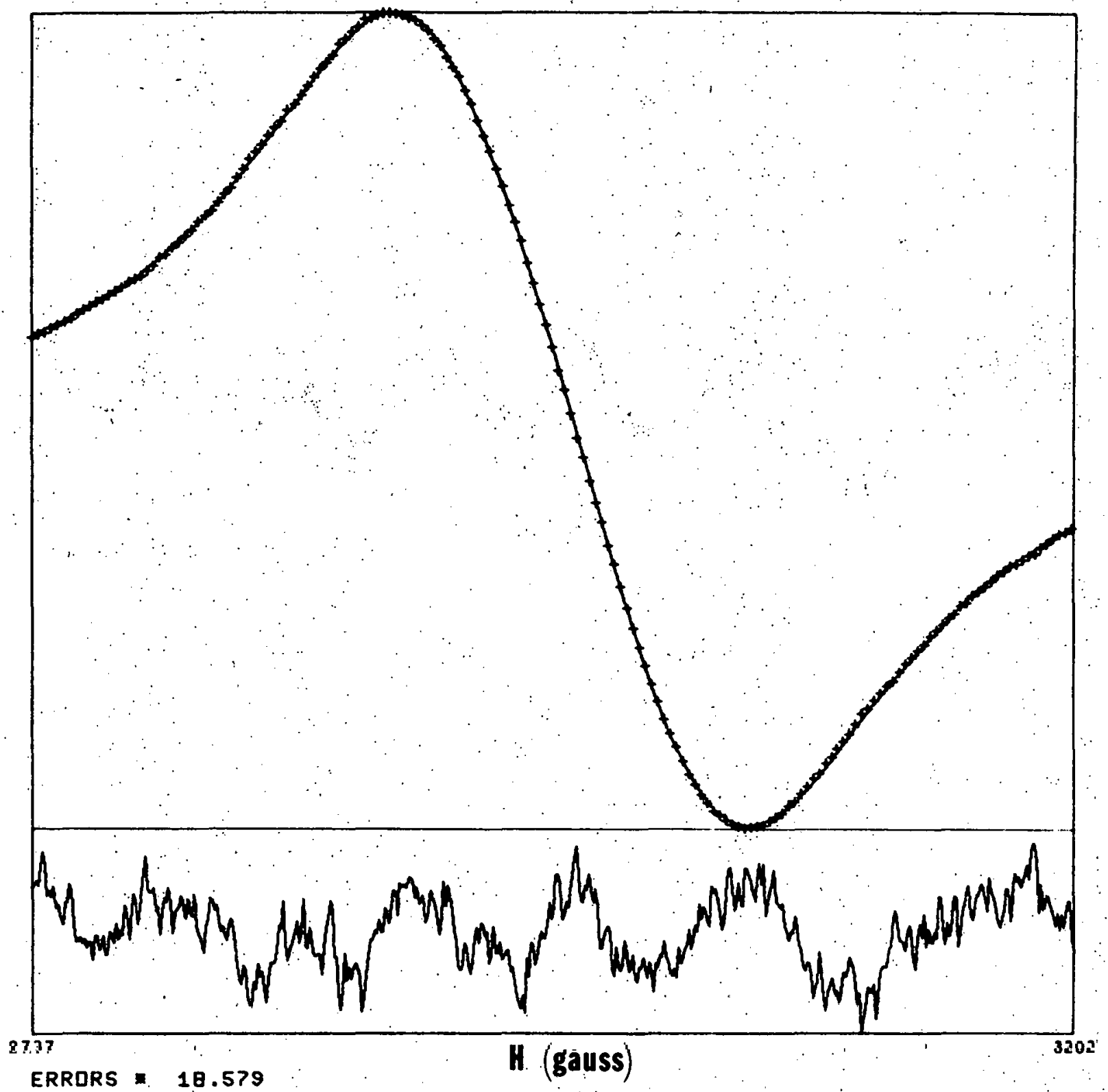

XBI. $7012-7173$

Fig. 19. Example of fit to solution spectrum of $\mathrm{Cu}\left(\mathrm{H}_{2} \mathrm{O}\right)_{6}^{2+}$ at $60^{\circ} \mathrm{C}$. 


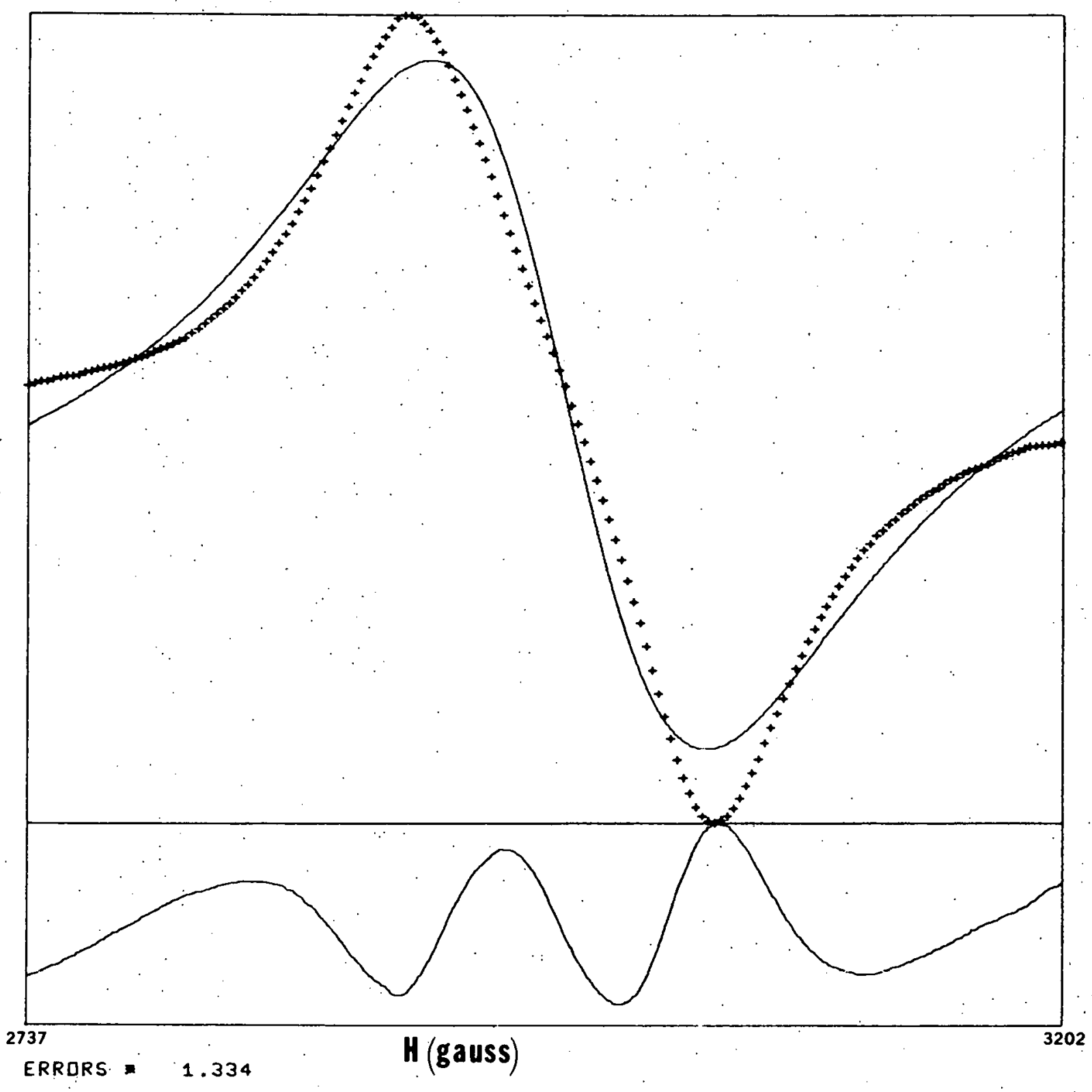

XBL $>012-7174$

Fig. 20. Attempt to fit spectrum of Fig. 18 with 1 Lorentz line. 


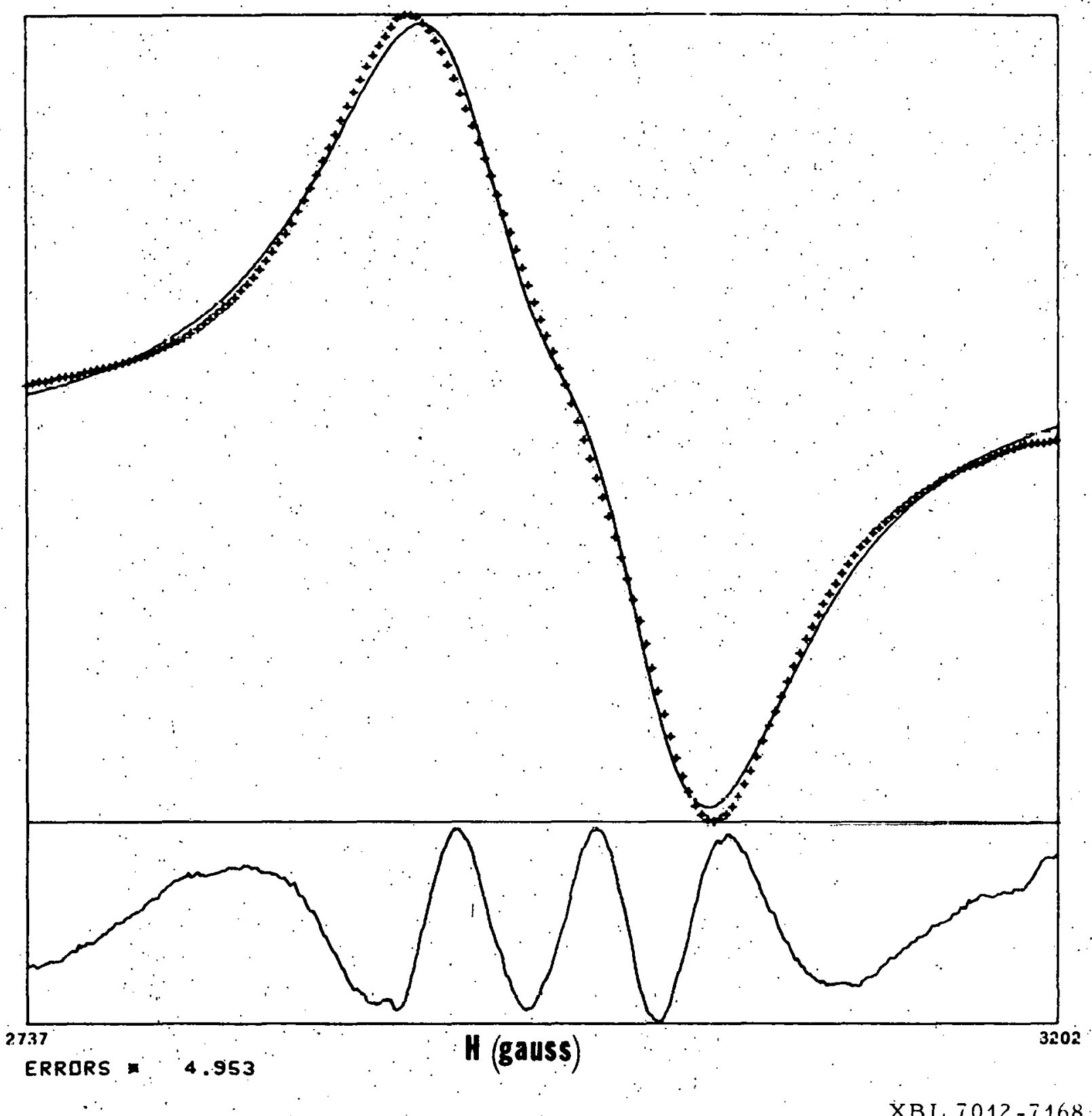

Fig. 21. Attempt to fit spectrum of FIg. 18 with 2 Lorentz Iines. 


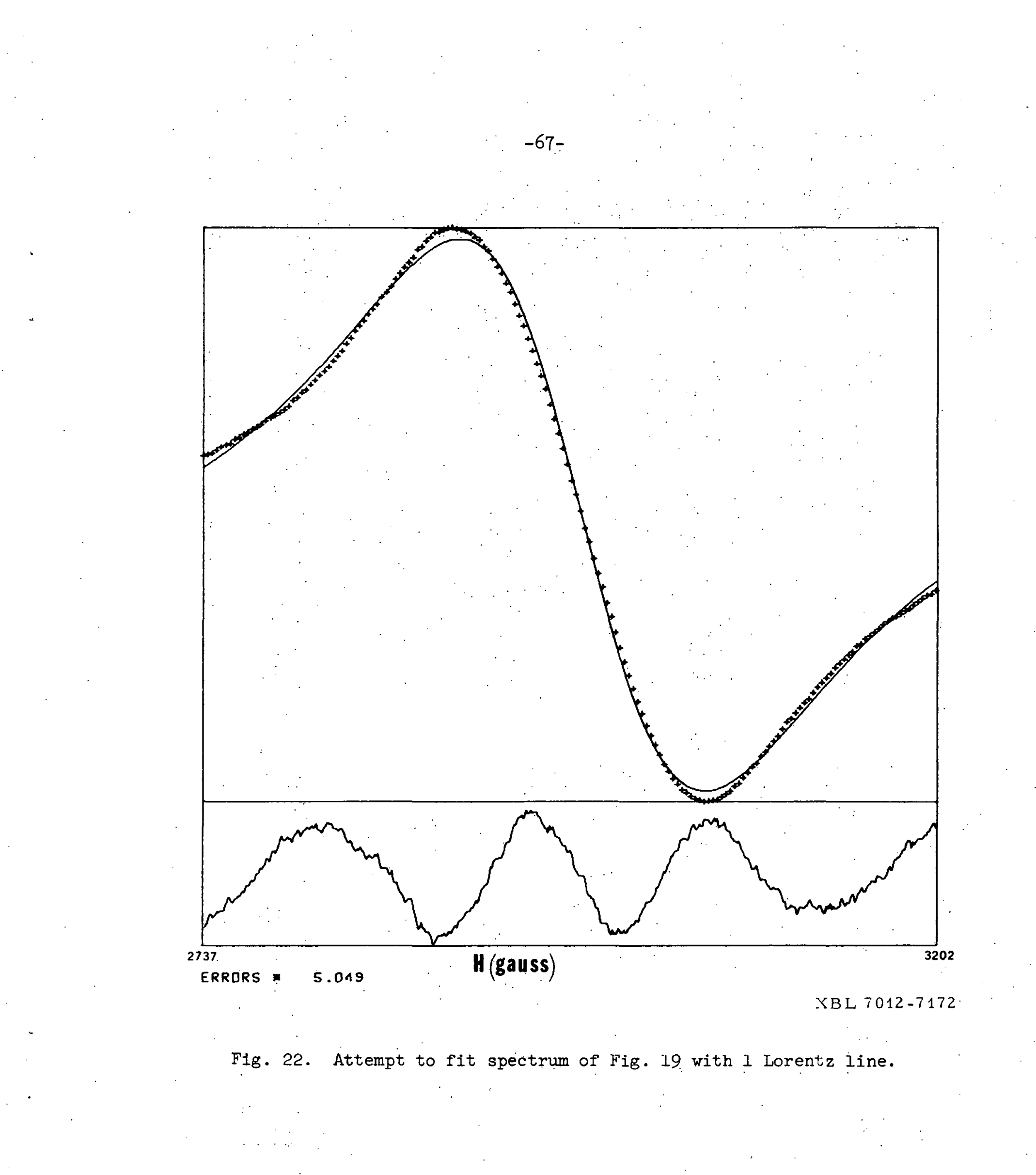




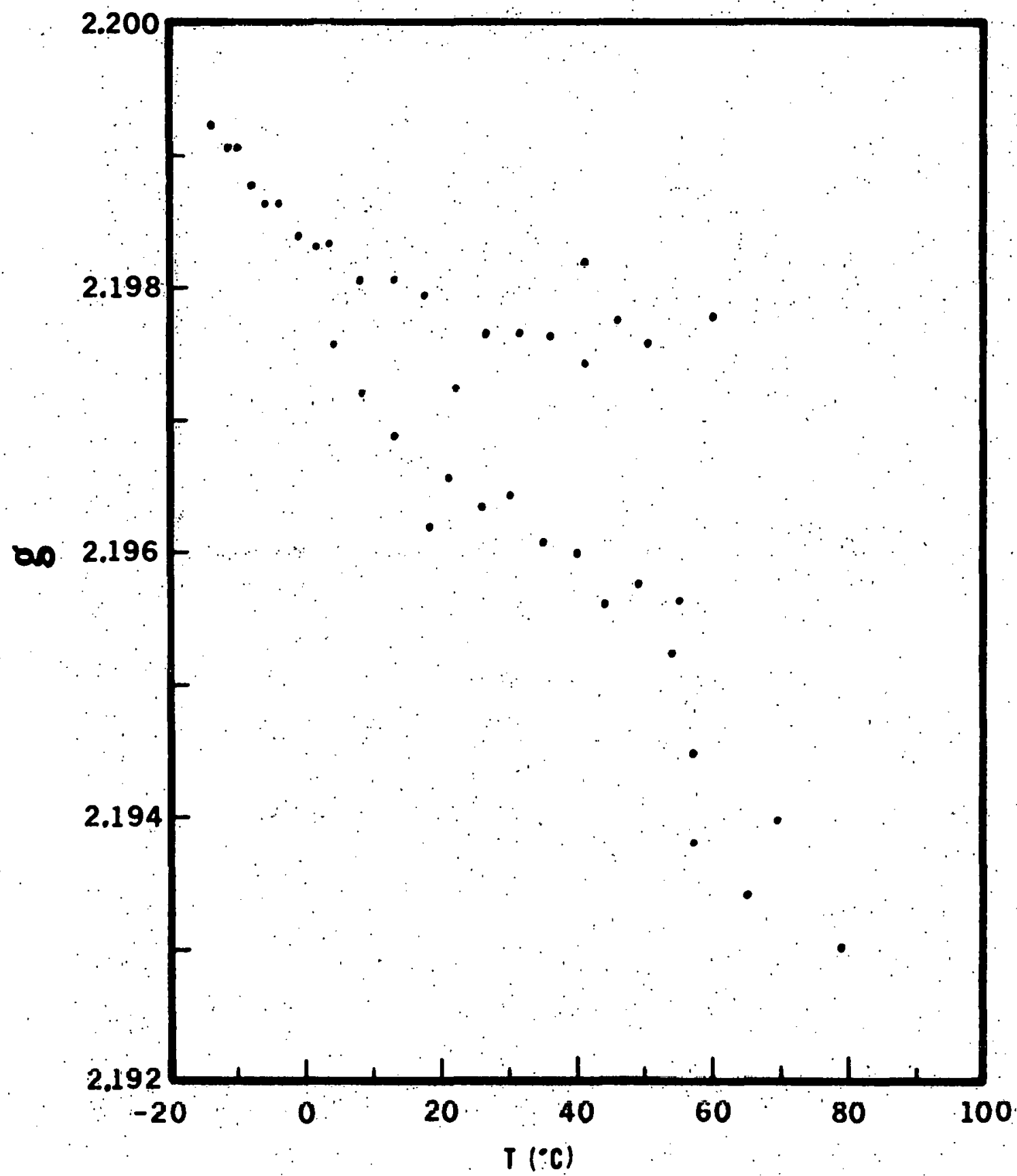

XBL $7.11-18$

Fig. 23. $g$ vs. $T$ for $\mathrm{Cu}\left(\mathrm{H}_{2} \mathrm{O}\right)_{6}^{2+}$ ? 


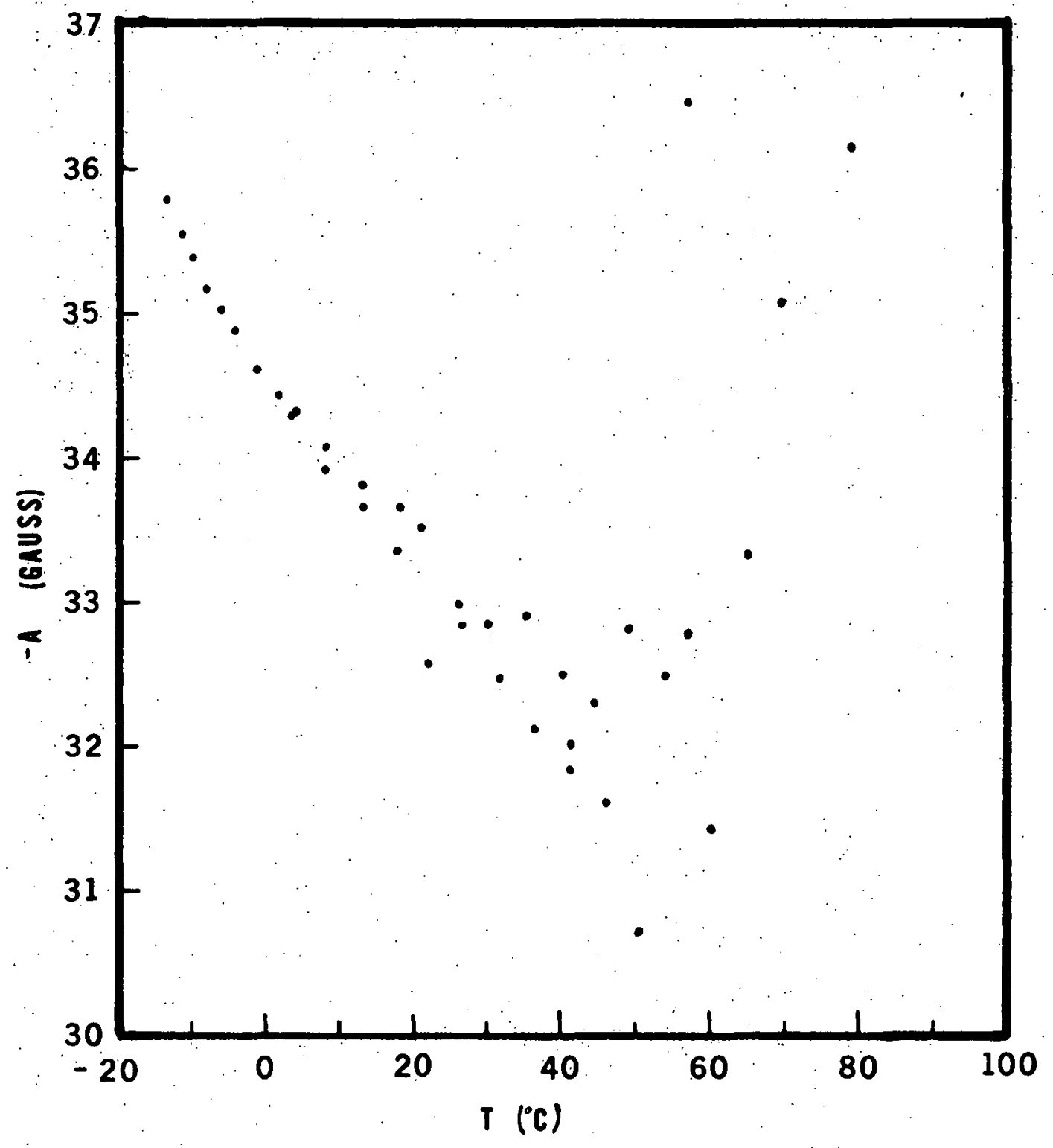

XBL 711-19

Fig. 24. -A vs. T for $\mathrm{Cu}\left(\mathrm{H}_{2} \mathrm{O}\right)_{6}^{2+}$. 
measure of the true width. Without knowledge of the low temperature spectra we would also be tempted to use the overall width as the true width. However, we can convince ourselves that this is incorrect by examining the spectra closely.

The results of an attempt to fit the room temperature spectrum with a single Lorentz line is shown in Fig. 20. This is the same spectrum that was presented in Fig. 18. The spectrum quite clearly does not possess a Lorentz lineshape. In fact close examination of the spectrum reveals several inflections near the center of the spectrum. This is shown more clearly in Fig. 21 which shows the result of fitting two Lorentz lines to the same spectrum. We may confidently infer from this that the observed spectrum consists of more than two lines. It is, therefore, quite reasonable to fit the spectrum with four hyperfine components.

Figure 22 shows the result of fitting the spectrum of F1g. 19 with one Lorentz line. Even at $60^{\circ} \mathrm{C}$ the spectrum is not Lorentz in shape. Hence, a Iineshape analysis indicates that the EPR spectra for hexaquocopper (II) must be analyzed in terms of four hyperfine components.

The variation with temperature of the isotropic and A values for hexaquocopper (II) is shown in Figs. 23 and 24. A variation of these parameters with temperature is not unexpected since such variations were observed for the aqueous vanadyl system and for vanadyl acetylacetonate (Wilson and Kivelson, 1966a). However, the usual observation is that the magnitudes of the $g$ and $A$ value change in opposite directions as the temperature is changed, that is, the $g$ value decreases as the A value increases. In the present case the $g$ and $A$ values change in the same direction. 
One might at first consider this effect to be an artifact because of the method of analysis. The line positions are determined by a second order spin Hamiltonian. The effect of the second order term is to shift all of the lines downfield. If the second order term is neglected, an apparent $g$ value is obtained which is higher than the true $g$ value. Furthermore, if the least squares fit erroneously causes the $A$ value to decrease, then, the shift due to the second order term would decrease and the apparent $g$ value would decrease. However, the shifts due to the second order term are on the order of a gauss and the changes in the second order term are somewhat less. Since the changes in the $g$ value correspond to shifts of several gauss, the effect must be real.

The usual interpretation of the temperature variation is that changes in solvation and bonding are occurring (Wilson and Kivelson, 1966a). However, this theory would require that the $g$ and $A$ values change in opposite directions since changes in bonding affect these parameters in opposite ways. Therefore, the variation in the hexaquocopper (II) system cannot be explained by this mechanism.

A number of mechanisms may be responsible for variations of the $g$ value or of the A value with temperature. Van Gerven, Talpe, and van Itterbeck (1967) have suggested a shift due to demagnetizing effects. They show that the shift in the $g$ value, $\Delta g$ is approximately given by

$$
\Delta g \sim\left(2 N_{s}-N_{1}\right) X_{s}
$$

where $N_{S}$ and $N_{1}$ are so-called demagnetizing factors, which are computed from the geometry of the sample, and $\chi_{s}$ is the static volume susceptibility of the sample. The $g$ value variation is expected to follow a Curie 
law. However, the magnitude of this effect is quite smail and would not account for the large $g$ value changes which are observed. Furthermore, this mechanism is not expected to cause a variation in the hyperfine coupling constant.

A second source of shifts are dynamic effects due to relaxation effects in solution. Such effects: have been discussed by Fraenkel (1967) and by Kivelson (1960). These effects are found in most treatments of relaxation theories but are generally Ignored by assuming that the static Hamiltonian can be redefined to include these terms (Slichter, 1963). The spin Hamiltonian is rarely redefined in practice. Kivelson derives a nonsecular shift to be

$$
\Delta=\tau_{c} \omega_{0} T_{1}^{-1}
$$

where here $\mathrm{T}_{1}^{-1}$ is the nonsecular contribution from the linewidth theory and is dependent upon $\mathrm{m}_{\mathrm{I}}$. The shift is then dependent upon the hyperfine component and follows a temperature dependence involving the correlation time and linewidth. Since the shifts are different for each hyperfine component, the hyperfine coupling constant may change as well as the $g$ value although the effect of the shifts on the least squares. determination is not clear. The effect of Eq. 4.3 can be estimated if the full linewidth is assumed to be due to $\mathrm{T}_{1}{ }^{-1}$. In fact the magnitude of the changes with temperature could account for the magnitude of the $g$ value changes. However, the computed shift is a downfield shift which increases with temperature. This should cause an increase of the $g$ value with temperature whereas the $g$ value decreases with increasing temperature. Hence, nonsecular shifts cannot be the reasons for the $g$ value variation. 
Another possible dynamic effect is the thermal vibrations of the ligands. Benedek, Englman, and Armstrong (1963) have studied the temperature dependence of IMR chemical shifts due to thermal vibrations. The effective crystal field splitting changes because the amplitudes of vibration change as a function of temperature. Unfortunately this effect would predict that the $g$ and $A$ values should change in opposite directions. Walsh, Jeener, and Bloembergen (1965) and Soos (1968) have studied the varlation of the $g$ tensor as a function of temperature in the solid state. Soos explains his variations for organic radicals in terms of delocalization of the electrons over radicals with slightly different $g$ tensors. As the temperature changes, the delocalization changes and the observed 8 tensor changes.. Walsh, et al., explain their behavior in terms of changes in the crystal field due to thermal expansion effects. In these experiments, the $g$ value decrease with increasing temperature was well explained by crystal field changes due to lattice expansion. However, Walsh observed that the hyperfine interaction (for $\mathrm{Mn}^{2+}$ ) decreased as the temperature increased, whereas the explanation for the 8 value change would predict an increase in the A value. Orbach (Simanek and Orbach, 1966; Calvo and Orbach, 1967) has studied this behavior and proposed that excited state configurations were mixed into the ground state by a "dynamic phonon-induced" field. This explanation was proposed for $S$ state lons but might be applicable to other states. Admittedly, these solid state explanations are not directly applicable to solution, but temperature dependent mixing of excited state configurations with the ground state is a possible explanation for the $g$ and $A$ value variation. Indeed, the hexaquocopper (II) complex is 
expected to have a low lying excited state due to the Jahn-Teller effect. This state could have an appreciable effect on the $g$ and A tensors.

The source of the variation of the $g$ and $A$ tensors is not well understood. Fortunately, the varlation in the parameters is relatively small so that the variation is neglected for the linewidth theories. The values of the isotroplc parameters used f'or the linewidth analysis are $g=2.1983$ and $A=-34.4$ gaüss.

\section{Relaxation}

The linewidths for hexaquocopper (II), which were also determined from the least squares fits, are presented in Figs. 25-28. The components are identified by $\mathrm{m}_{I}$, the nuclear magnetic quantum number, and since the hyperfine coupling constant is assumed to be negative, the $m_{I}=-3 / 2$ line is the lowest field Iine and the $m_{I}=+3 / 2$ line is the highest field line. The linewidths for the components show a smooth functional dependence on the temperature, increasing monotonically with the temperature. "Below $40^{\circ} \mathrm{C}$ the deviations in the data are small. Above this temperature, there is increasing scatter in the data. At the lowest temperatures where hyperfine components are distinctly visible, the linewidths are known to about .5\%. Near room temperature where the line is unresolved, but definitely not lorentzlan, the exror is expected to be sbout $1 \%$. There is much less confidence in the data at higher temperatures where the line is near Lorentzian, and the error is expected to be several per cent. The dashed curves in the figures are arbitrary interpolation curves.

The linewidths for the hyperfine components are compared in Fig. 29. Examination of the low temperature region reveals a distinct dependence on hyperfine component, with the narrowest line being the $m_{I}=+3 / 2$ or 


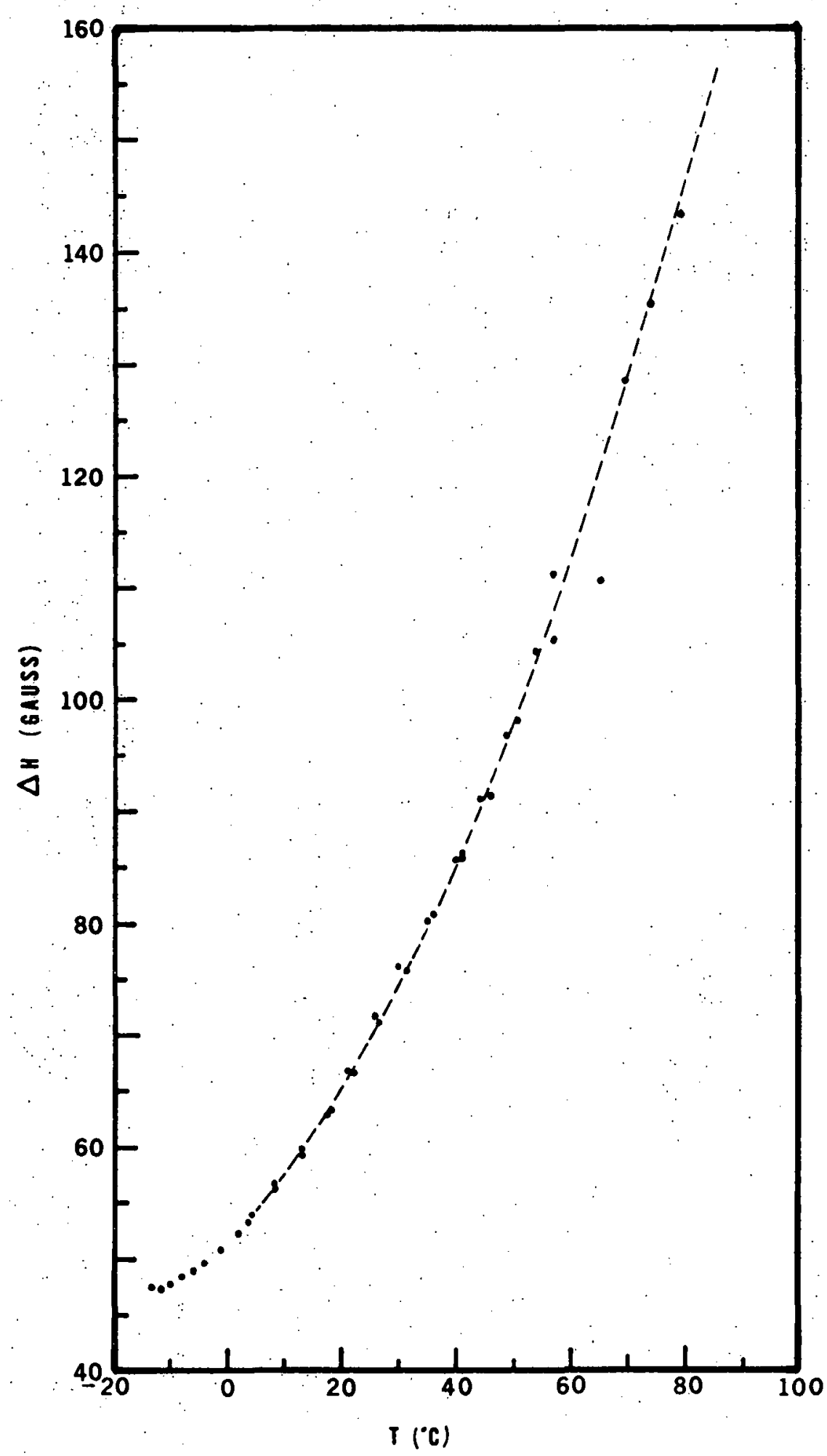

XBL. $7012-7490$

Fig. 25. Peakto-peak linewidth of $-3 / 2$ component vs. T. Dashed curve is interpolation graphical. 


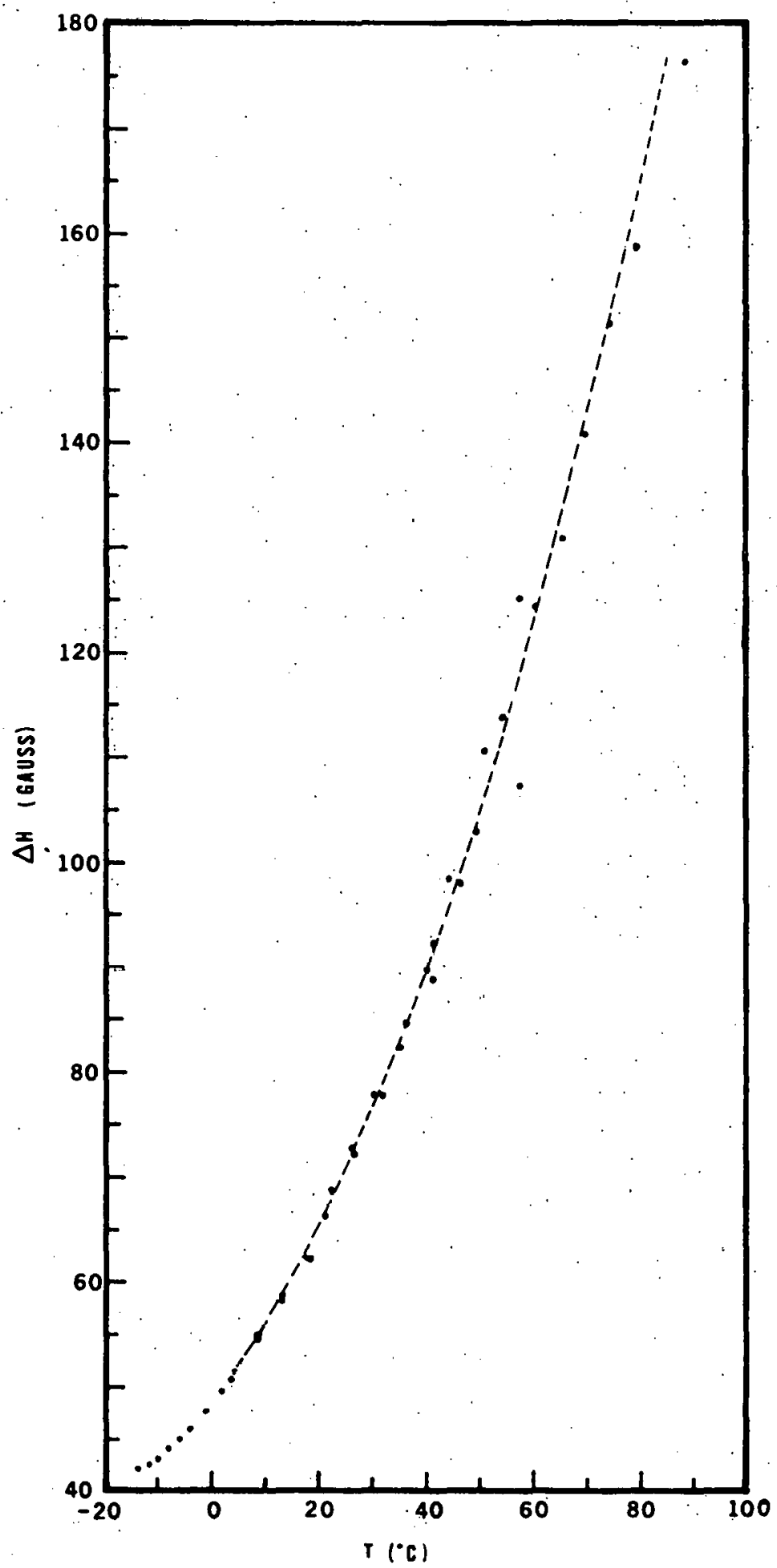

XBL 7012-7493

Fig. 26. Peak-to-peak linewidth of $-1 / 2$ component vs. T. 
$-77-$

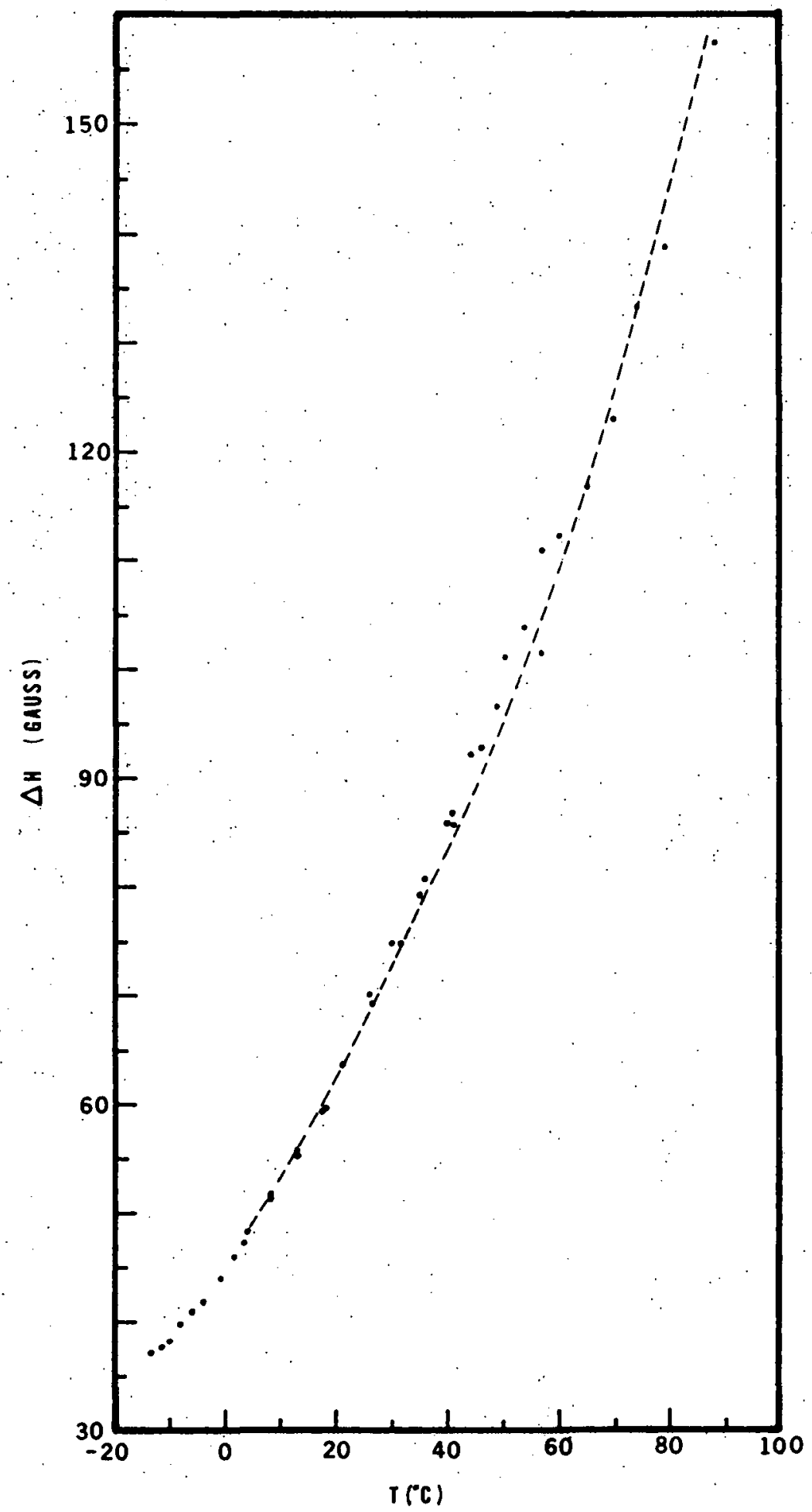

XBL $7012-7489$

Fig. 27. Peak-to-peak linewidth of $+1 / 2$ component vs. T. 


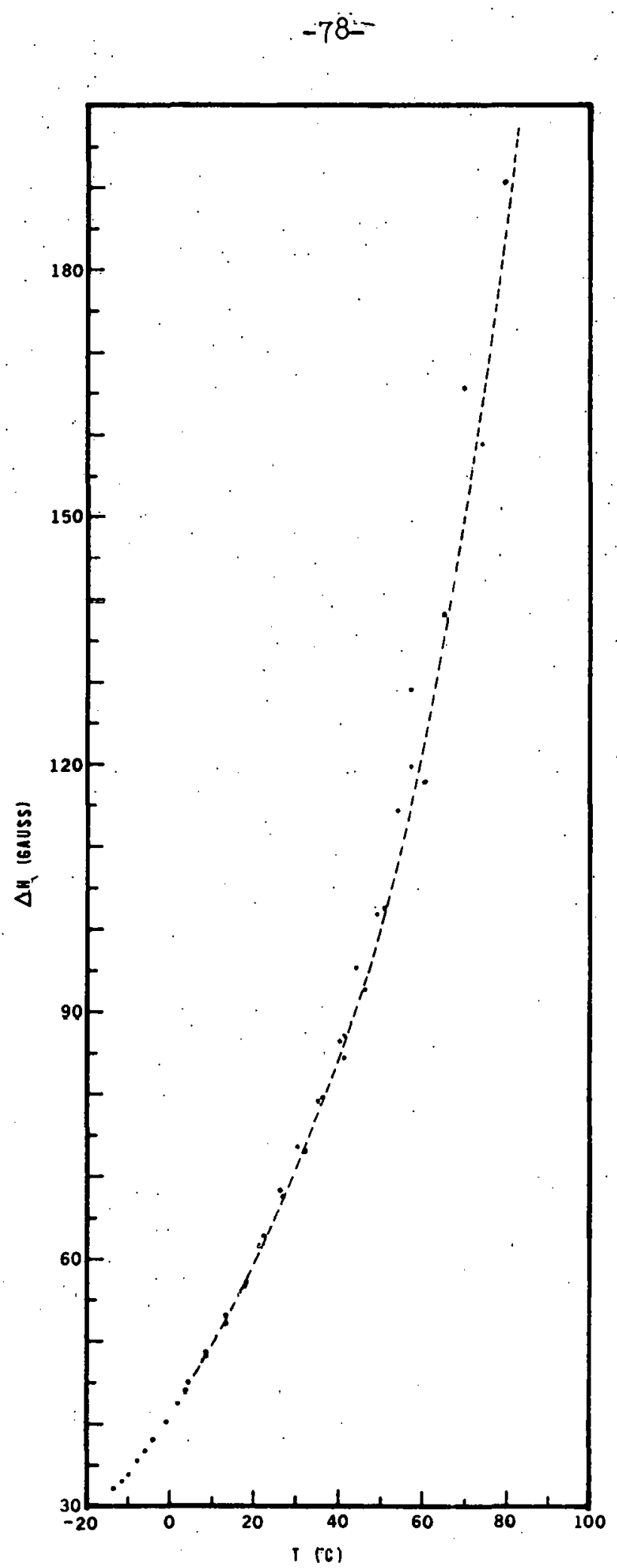

XBL 7012-7491

Fig. 28. Peak-to-peak linewidth of $+3 / 2$ component vs. T. 


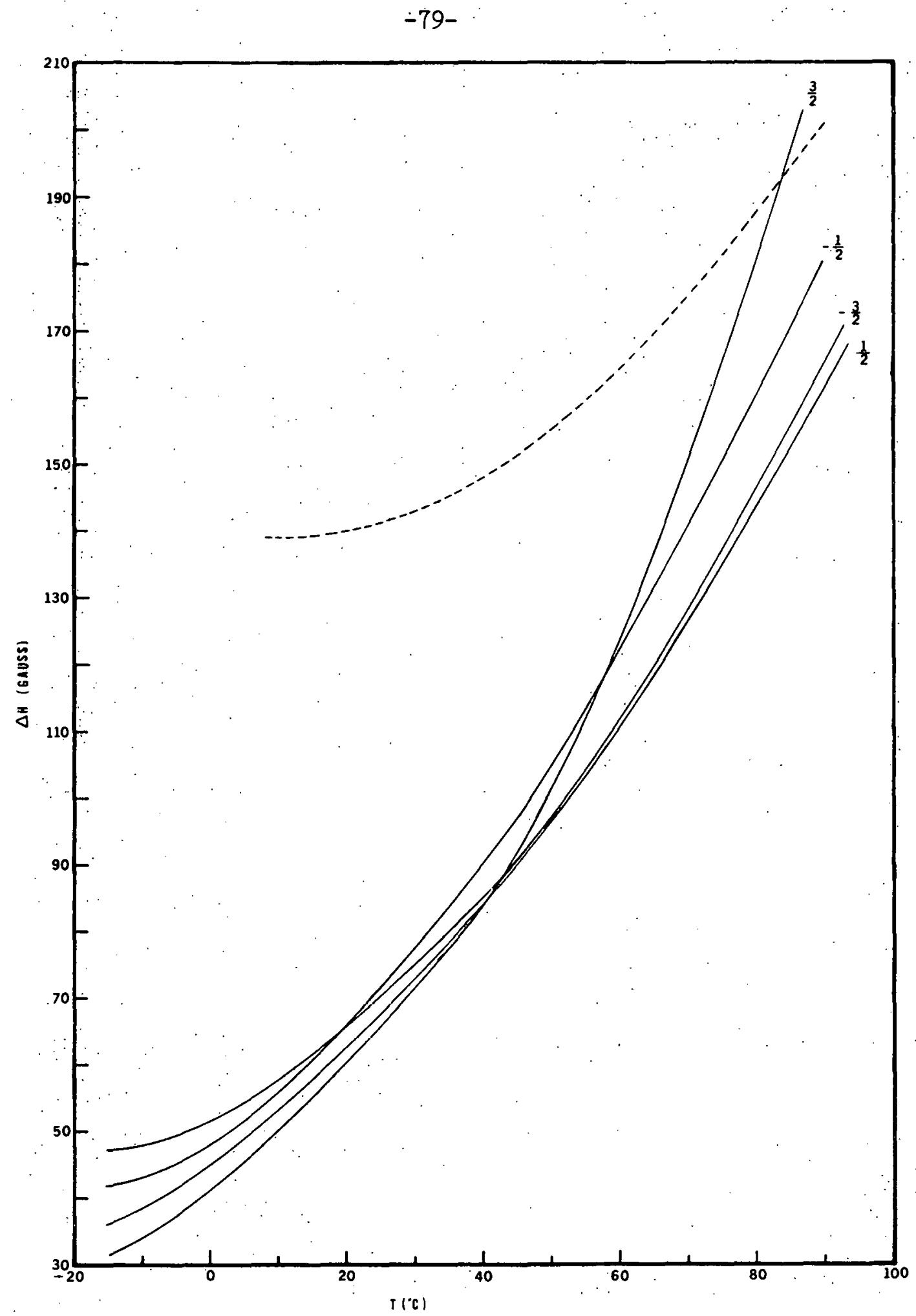

XBI $7012-7492$

Fig. 29: Comparison of Iinewidths for hyperfine componente of $\mathrm{Cu}\left(\mathrm{H}_{2} \mathrm{O}\right)_{6}^{2+} \ldots$ 
highest field line. The lines are progressively broader as $m_{I}$ is decreased, with $m_{I}=-3 / 2$ as the broadest ine. As the temperature is increased, the relative widths change until the $m_{I}=-1 / 2$ line becomes the broades: line. At still higher temperatures, the $\mathrm{m}_{I}=+3 / 2$ ine appears to becolne the broadest line. We are not certain that this behavior is real. At the high temperatures least squares procedures are quite difficult. Noise and drifts in the spectrum could cause large. changes in the least squares fits. However, the linewldths definitely depend on the hyperfine component at low temperatures and appear to depend on the hyperfine component at higher temperatures.

The dashed Iine in Fig. 29 is the linewidth determined from the overall peak-to-peak distance of the unresolved line. The fallacy of using the overall width as the true width is clearly seen. The temperature dependence and the magnitudes of the width are incorrect. Valiev and Zaripov (19.66) indicate that their mechanism is consistent with this data, so that the mechanism is probably incorrect for the copper system.

The dependence of the linewidths on hyperfine component leads one to suspect a Kivelson tumbling mechanism (Wilson and Kivelșon, 1966a) to be contributing to the linewldth. In accord with this idea, the linewidhs were fit to a cubic polynomial. The polynomial is exactly determined by four linewidths, so that the polynomial coefficlents may be strongly influenced by small errors in the linewidths. The parameters which were determined are presented as the points in Figs. 30-32. The alpha parameter appears to vary quite smoothly with temperature. The beta and gamma parameters exhibit much scatter which is extreme at high temperatures. The delta parameter is even worse (not presented). (The correlation of temperature with viscosity divided by temperature is presented in Table II.) 
As was done with the vanadyl data, the beta parameter was used to determine a hydrodynamic radius for the complex using Eqs. 2.6 and 2.3. The theoretical values for $\alpha^{\prime}$ and $\gamma$.were then computed from Eqs. 2.5 and 2.7 and are presented as the smooth curves in Figs. 30-32. These curves were computed using the anisotropic magnet1c parameters which were determined in this work (see Sec. IV.C.I). The curves computed with the Lewis, Ale1, and Morgan parameters are quite similar, and, therefore, are not plotted. We can see immediately that the theoretical and experimental parameters are in marked disagreement. The beta fit is relatively good at low temperatures. However, the experimental values for the gamma parameter are negative until the lowest temperatures are reached. The theory predicts that the gamma parameter should be positive over the entire temperature range. The theoretical prediction of the alpha parameter is also far from agreement with experiment.

Furthermore, the values of the correlation time and the hydrodynamic radius which are determined from the fit to the beta parameter are anomalously low. The radius, $r=1.71 \mathrm{~A}$, which was determined using the anisotropic parameters from this work, is of the order of the ionic radius for copper. If the Lewis, Alei, and Morgan (1966) magnetic parameters are used, the radius is $r=1.83 \mathrm{~A}$, which, although better, is still too small. By analogy to the vanadyl system, the radius is expected to be on the order of $3 \mathrm{~A}$. Cox and Morgan (1959) and Morgan and Nolle (1959) have measured the proton NMR for copper solutions. The proton relaxation is due to a dipolar interaction modulation by molecular tumbling, so that the correlation time is related to the hydrodynamic rulius by Eq. 2.3. These workers obtain a radius $r=2.8$ A. Frankel (1968) in a more recent study obtained $r=2.3 \mathrm{~A}$. 
The validity of the Kivelson tumbling model for the hexaquocopper (II) system is doubtful. Further evidence is obtained from the dependence of the theory on magnetic field. The EPR spectrum for hexaquocopper (II) was measured at S. band $\left(3.12 \mathrm{GHz}\right.$ ) at room temperature (about $22^{\circ} \mathrm{C}$ ). The spectrum is presented in Fig. 33. The linewldths obtained from a least squares fit to the $S$ band spectrum are $67.8,66.9,65.7$ and 65.9 zauss, respectively, for the $-3 / 2$ to $+3 / 2$ component. The corresponding linewidths for the $X$ band spectrum are 66.6, 67.7, 64.1, and 62.0 gauss (from Fig. 29). Although there is a definite dependence upon hyperfine component, there is relatively little difference between the $\mathrm{S}$ band and $\mathrm{X}$ band linewldths. The Kivelson theory would predict a strong dependence of the linewlaths with magnetic field. In addition, if we ignore these problems and continue to use the Kivelson mechanism, we may compute a linewidth contribution from the spin-rotation mechanism. If this is done, we compute linewidth contributions which are flve limes the experimental linewidths. Therefore, this procedure cannot be used to explain the linewiaths, although it was very successful for the vanadyl case.

We must, theref'ore, proceed in another manner to expluin the linewidths. The increase of the linewidths with temperature strongly indicates. a spin-rotation mechanism. Ignoring the $m_{I}$ dependent terms for the present, we plot the $m_{I}$ independent linewidth, the alphe term, vs. T/n in Fig. 34. The alpha term is quite linear with temperature divided by viscosity. (The alpha term has not been corrected for a contribution from the tumbling mechanism because of the uncertainty with the theory). This is consistent with the prediction of a spin-rotation mechanism except that the extrapolation of alpha to zero temperature does not pass through zero. Nevertheless, we may use the slope of a straight line fit to the 
data with Eq. 2.12 to determine a hydrodynamic radius of $r=3.08 \mathrm{~A}$. This is a reasonable value.

If we assume the spin-rotation mechanism to be dominant, we may compute a contribution from the tumbling mechanism using the new hydrodynamic radius. If this is done, the predicted linewidth parameters are found to be much larger than the experimental parameters. Since we expect the tumbling mechanism and the spin-rotation mechanism to be consistent with each other, we must conclude that these mechanisms cannot both be fully operative in the hexaquocopper (II) system.

Lewis, Alei, and Morgan (1966) propose that the anisotropy of the copper complex is less in the aqueous solution than in the glass. They reduce the anisotropies, $\Delta g$ and $b$, and compute a contribution of the tumbling mechanism to the linewidth. After correcting the $\mathrm{m}_{I}$ independent term for the tumbling contribution, they fit the remainder to a spinrotation mechanism. They also include a term quadratic in temperature which they describe as a Raman process (see Ea. 4.I). This term is necessary to account for the curvature which appears in the alpha term after the tumbling correction is made.

The Raman mechanism was proposed on the basis of an extrapolation from the solid state work of Gill (Gill, 1965; Stoneham, 1965) on copper ions in Tutton salts: However, the more recent calculations of Kivelson (1966) have shown that the contributions of the Raman process in liquids is small. Therefore, although Lewis, Alei, and Morgan fit the $\mathrm{m}_{\mathrm{I}}$ independent term well with a spin-rotation interaction and a $\mathrm{T}^{2}$ term, the Raman process is not indicated.

Furthermore, Lewis, et al., are slightly inconsistent in their adjustment of the anisotropies. Although they adjust the anisotropies 
to compute the tumbling width, they do not adjust the $g$ values in computing the spin-rotation width: Furthermore, they show that the magnitude of the ${ }^{17} 0$ coupling constant, which they measure from temperature dependent shifts of the ${ }^{17}$ O MMR line, is consistent with the anisotropic parameters determined in a glass. In addition, they argue from Stoneham's work (Stoneham, 1965) that the first orbitally excited state for the hexaquocopper (II) complex is expected to be $7700 \mathrm{~cm}^{-1}$ above the ground state. This is consistent with a large tetragonal distortion and, hence large anisotropy.

The Inewidths in hexaquocopper. (II) are then somewhat of a mystery. The solution Raman process is unlikely. The solution Orbach process is unlikely if the lowest exclted state is $7700 \mathrm{~cm}^{-1}$ above the ground state. The increase of the linewidth with increasing temperature is most likely explained by a spin-rotation interaction. However, the contribution from a tumiling mechanism consistent with the spin-rotation interuction and with the large anisotropy is too large. The tumbling mechanism cannot contrlbute its full effect and must be interrupted by some other relaxation process which is also $m_{I}$ dependent. Ihis would be something simllar to the inversion mechanism discussed by Spencer (1965). Spencer treated' both mechanisms separately. A more proper treatment is probably to treat both the tumbling process and the inversion process together. The linewidths in hexaquocopper (II) are then due to a spin-rotation interaction, a tumbling mechanism, and a third process, similar to inversion, which interrupts the tumbling mechanism, and which is essentially independent of magnetic field. 


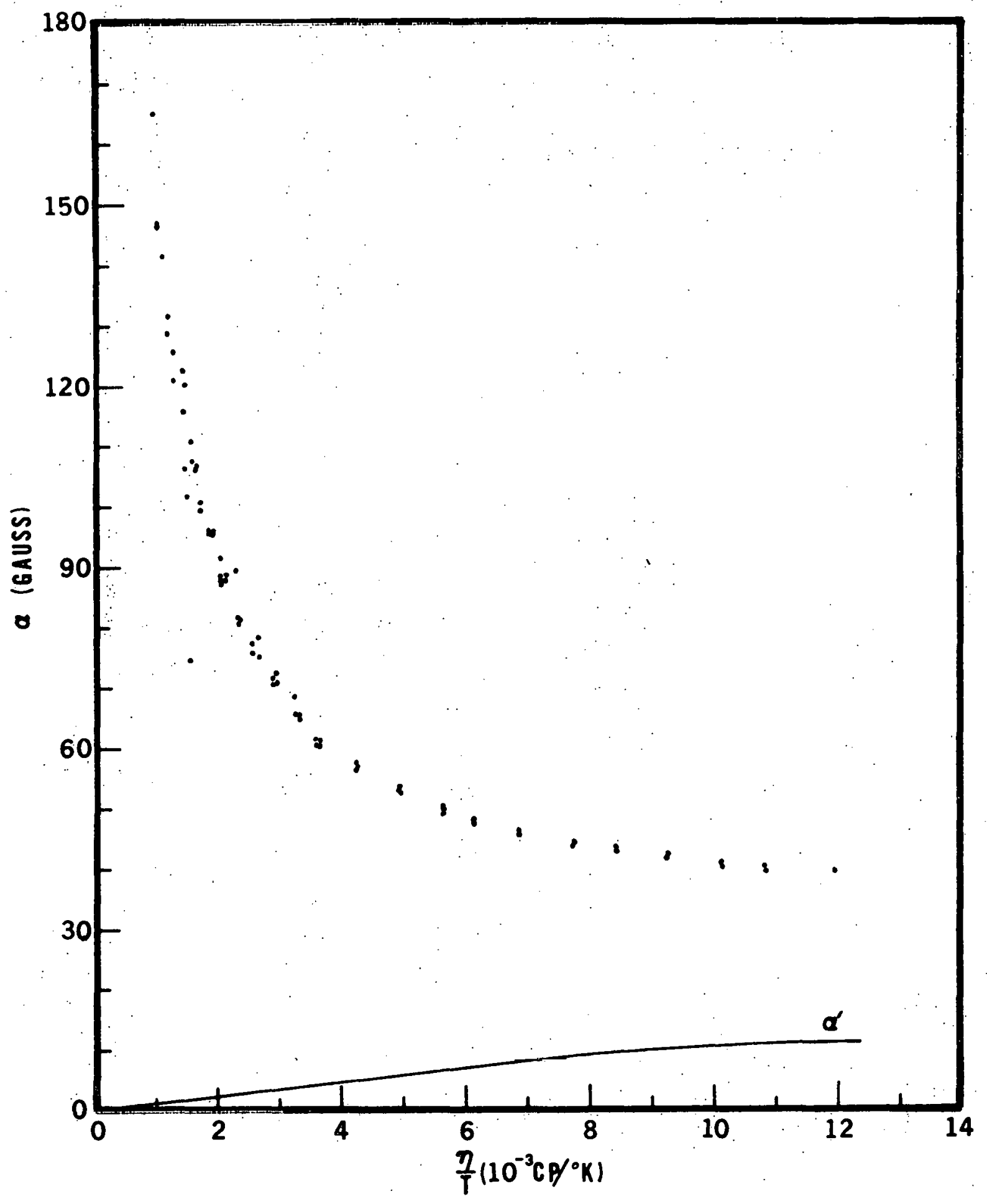

XBL $711-15$

Tig. 30. Alpha parameter vs. $\mathrm{n} / \mathrm{T}$ for $\mathrm{Cu}\left(\mathrm{II}_{2} \mathrm{O}\right)_{6}^{2+}$. 


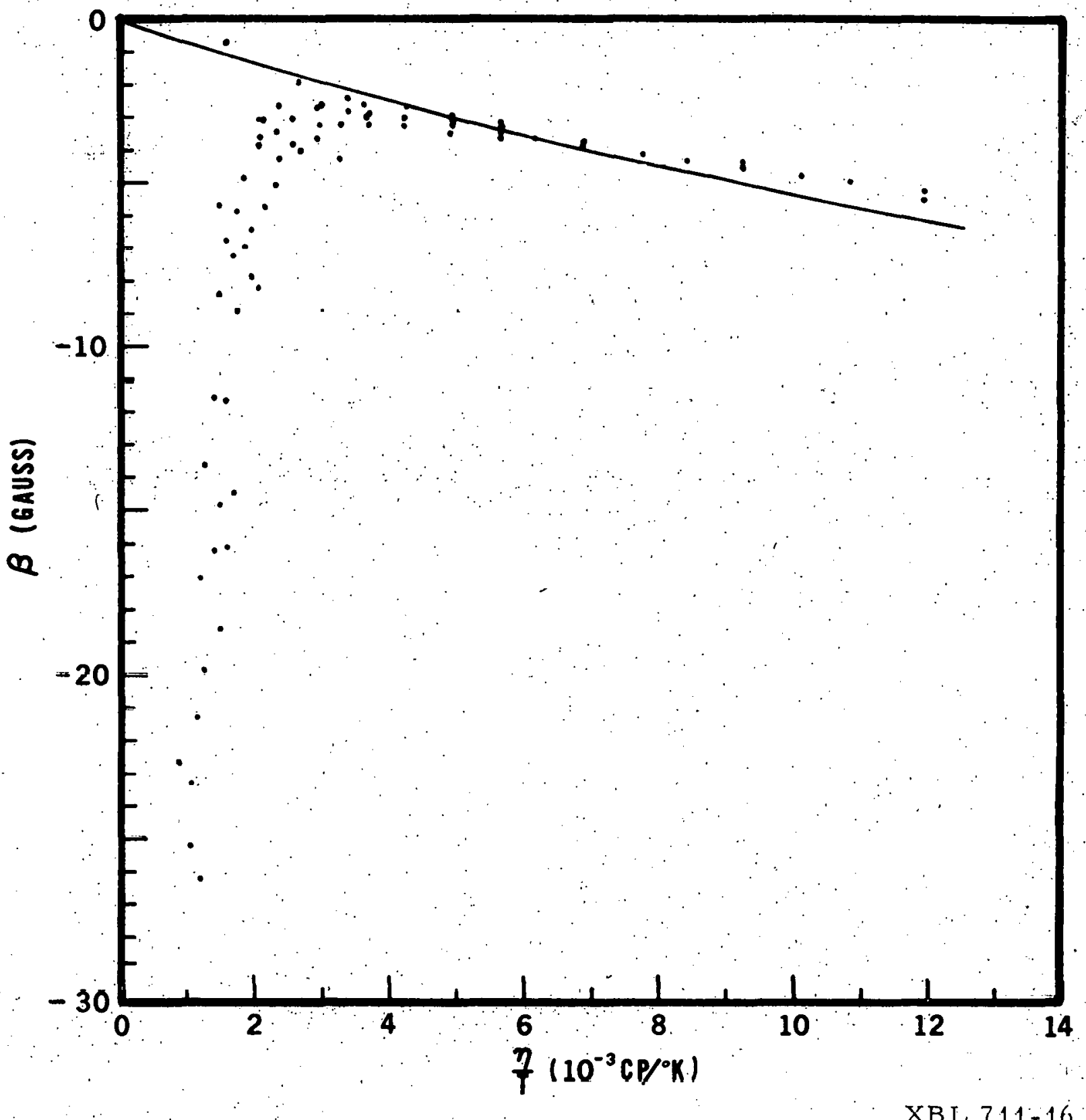

Fig. 31. Beta parameter vs. $i / T$ for $\mathrm{Cu}_{2}\left(\mathrm{H}_{2} \mathrm{O}\right)_{6}^{2+}$. 
$-87-$

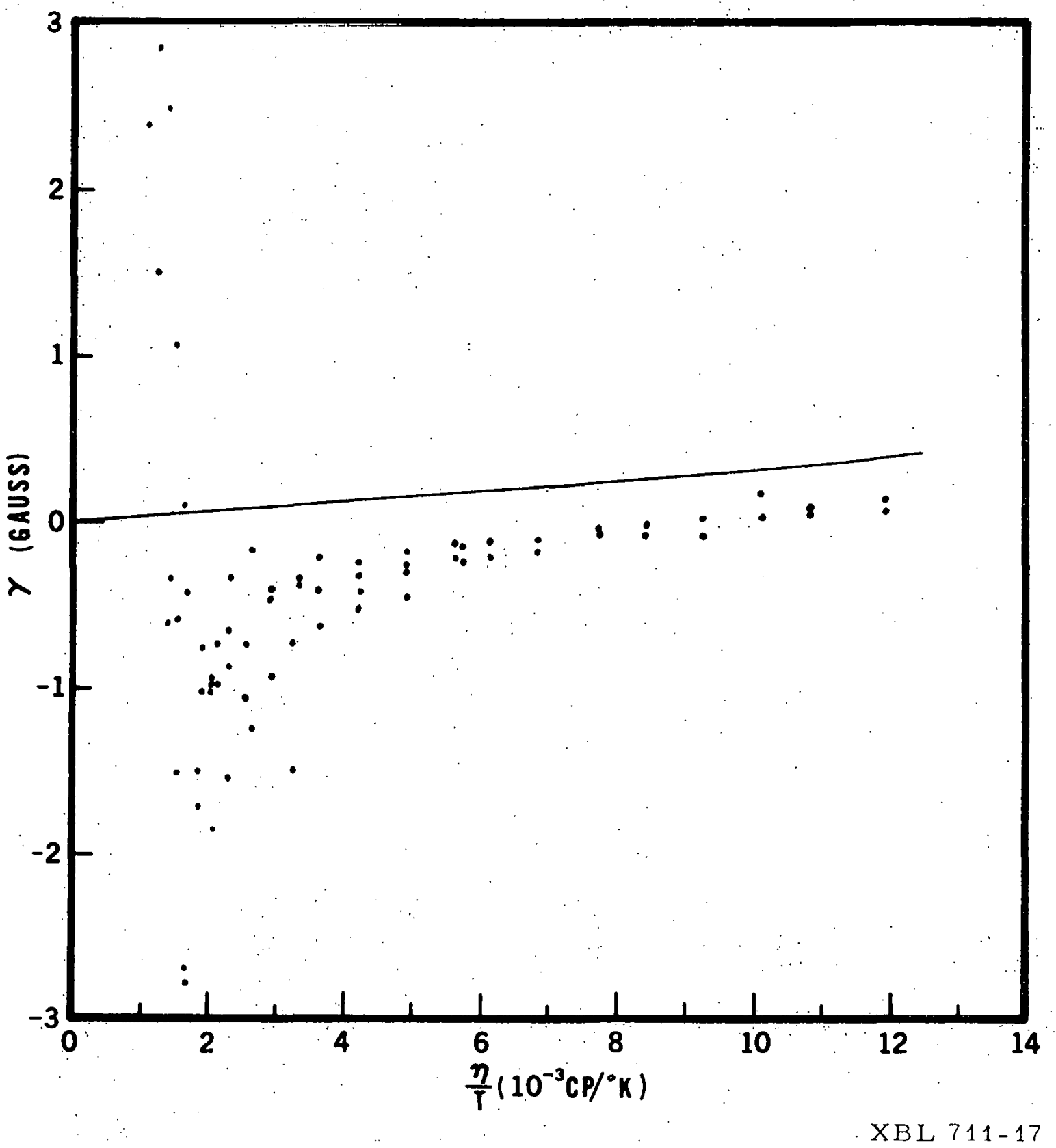

Fig. 32. Gamma parameter vs. nIT for $\mathrm{Cu}\left(\mathrm{H}_{2} \mathrm{O}_{2}\right)_{6}^{2}$. 
$-88-$

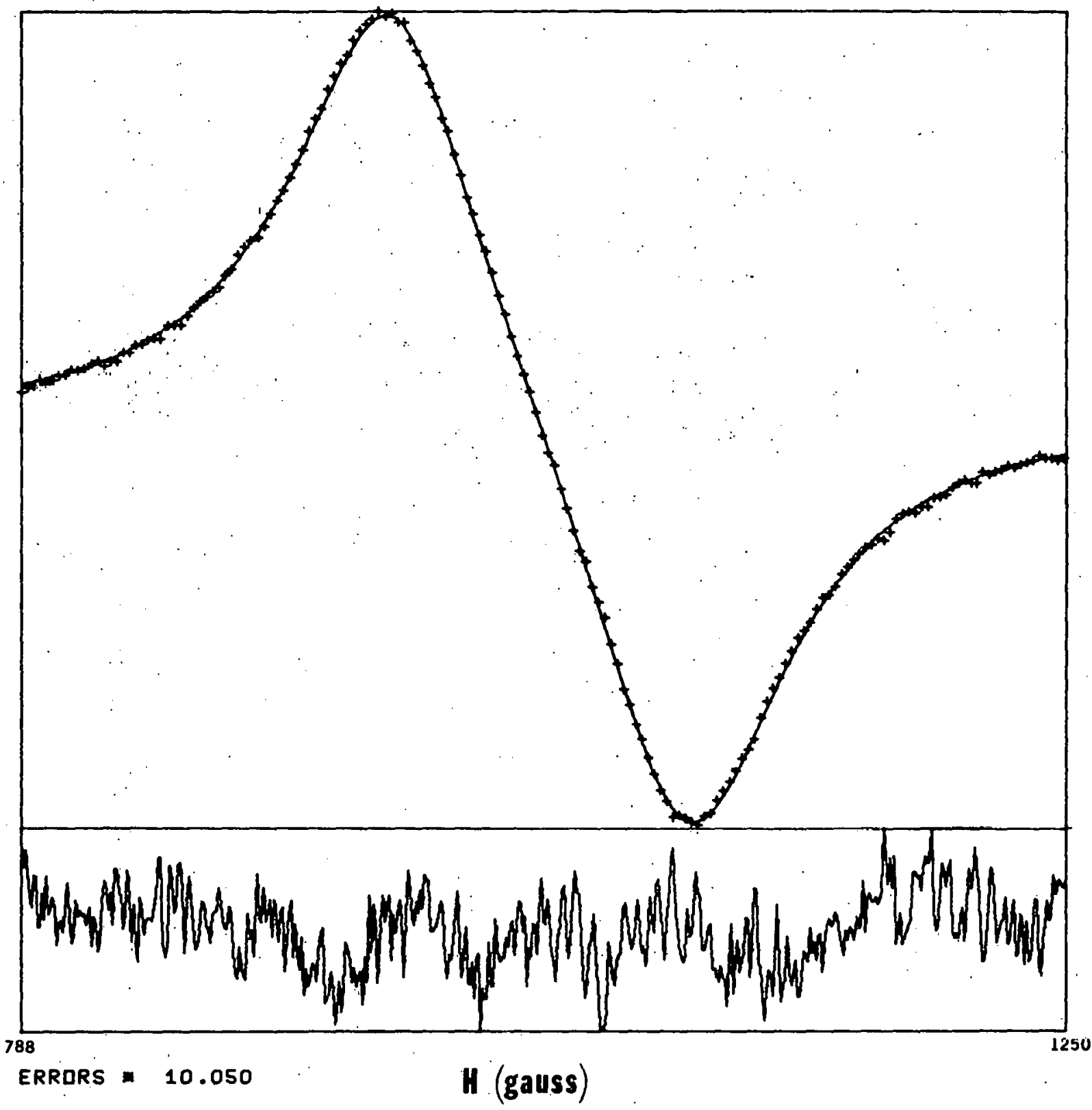

X BL $7012-7320$

Fig. 33. Example to fit to solution spectrum of $\mathrm{Cu}\left(\mathrm{H}_{2} \mathrm{O}\right)_{6}^{2+}$ at room temperature with a microwave frequency of $3.12 \mathrm{GHz}$. 


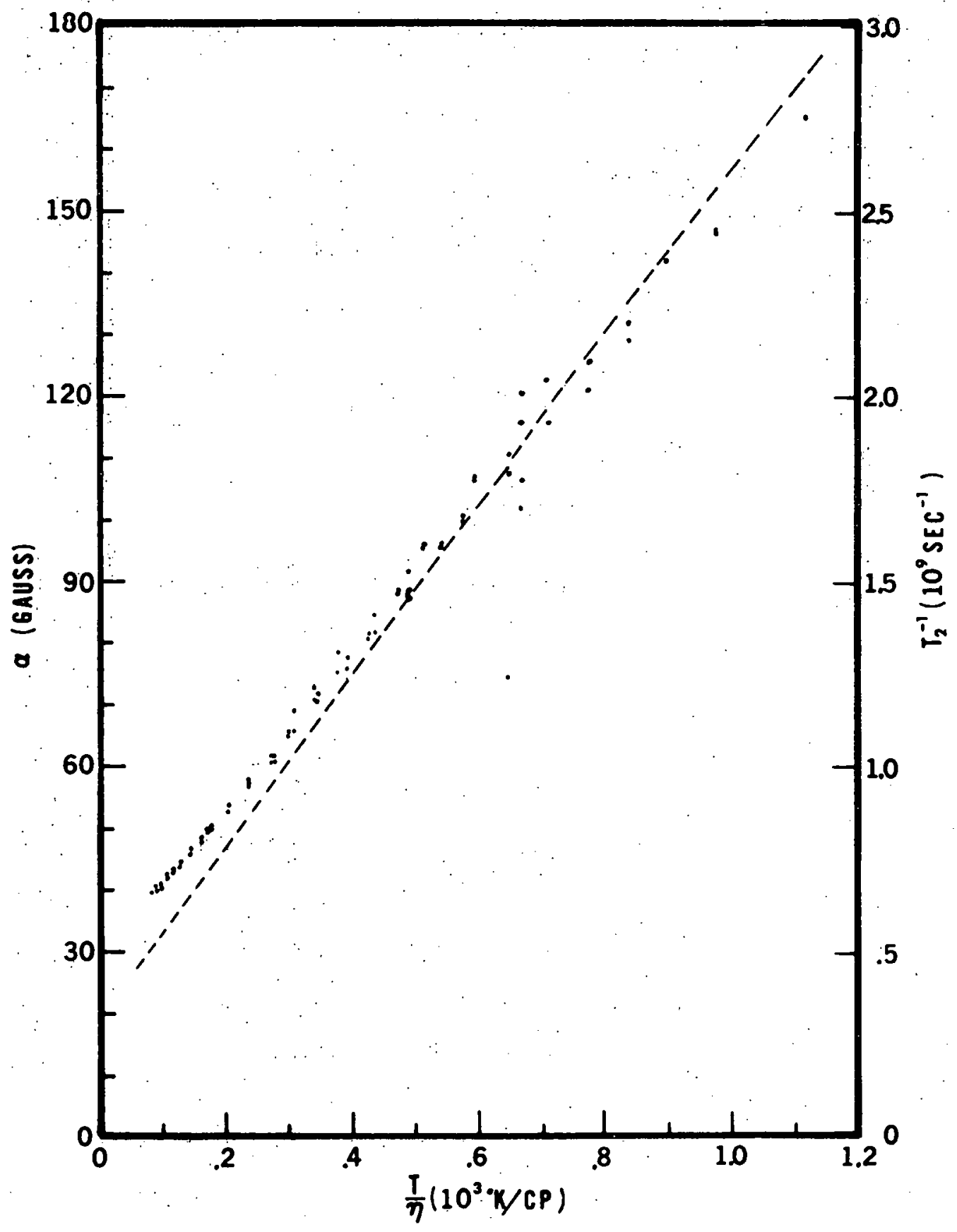

XBL $711-95$

Fig. 34. Alpha parameter vs. I'/n for $\mathrm{Cu}\left(\mathrm{H}_{2} \mathrm{O}\right)_{6}^{2+}$. The dashed curve is plotted with Eq. 4.1. (see text). 


\section{SPIN-IATTICE RETAXATION TIME MEASUREMENTS WITH AN ON-LINE COMPUTER}

\section{A. Introduction}

The longitudinal or spin-lattice relaxation time is extremely important in magnetic resonance, for magnetic resonance phenomer a would not be observable if satisfactory relaxation mechanisms did not $\epsilon$ xist. The spin-lattlce relaxation times of transition metal ions diluted in various host lattices is of considerable interest. A knnwlerge of these times aids in the theoretical understanding of electron paramagnetic resonance and in the interpretation of the interactions which may occur in solids. Furthermore, the relaxation times which are related to transition probabilities are important in understanding the operation of masers (BIoembergen, 1956). Stevens (1967) and Standley and Vaughan (1569) have recently reviewed the theoretical and experimental aspects of the spin relaxation measurements.

A number of techniques have been developed for the measurem ent relaxation times. One of the most popular is the pulse saturaticn-recovery method (Davis et al., 1958). In this method the spin system to be studied is saturated with a relatively high power nulbe; and the recovery of the absorption slgnal as a function of time is monitored; usually on an osc1lloscope. 'Ihe relaxation time is determined either by measuring the slope of a plot of the lograithm of the recovery curve or by comparing the recovery curve with standard exponentials whose time constants are known. The logarithmic method requires a knowledge of the baseline to which the recovery curve is returning. This is done experimentally by providing a second scan at low power to show the recovered signal on the 
oscilloscope. This could involve experimental complications. The comparison method suffers if the recovery curre is not a single exponential. Furthermore, in many cases, the recovery curve contains a large amount of noise which makes the measurements from the oscilloscope traces difficult and inaccurate. Much experimental effort is usually expended to reduce this noise. Indeed, the improvement of the signal-to-noise ratio is one of the most serious problems in experimental science.

A technique for improving signal-to-noise is that of signal averaging. The usual method is to use a highly specialized computer of averaged transients (CAT) to acquire the data. Successive recovery curves are accumulated whth the CAT until the signai-to-noise is acceptable. The data are then usually printed for analysis by the techniques described above, or punched for analysis with a digital computer.

However, we had been interested for some time in the application of small computers to laboratory problems. The advantages of using a computer are now well known. Interest in the technique has grown to such an extent that the January, 1969, issue of the IBM J. of Research and Development is devoted to laboratory automation. The problem of spinlattice relaxation time measurements appears to be tailor made for a small computer. Not only can the data be acquired with the computer, for which time is a natural independent variable, but the data analysis can also be performed. In the case of simple exponential recoveries the analysis is straightforward and easily automated. Baseline problems are mand hy halled afmply by acquifing data out to tims whore the baseline is well defined. More complicated recovery curves may be analyzed by an 
interactive technique using relatively simple conversational programs and a display oscilloscope. An additional advantage of a computer is that an analysis of the data can be performed quickly so that results are known instantly.

The $\mathrm{Ni}^{2+}$ Ion was chosen for study since this was a transition metal Ion of current interest in the laboratory (Jindo, 1971 ; Batchelder, 1969 ). of interest is the $D$ value which is negative for most nickel compounds. However, in nickel sulfate the $D$ value is positive. This behavior is discussed by Jindo, 1971. Studles of nickel ions in other lattices were undertaken to obtain information to aid in a theoretical interpretation of this behavior. The EPR spectmum of nickel ion in a lanthanum magnesium nitrate host (Ni/LMN) has been studied in this laboratory (Jindo, 1971) and was selected as a suitable system in which to study relaxation times. Furthermore, relatively few measurements of relaxation times in nickel ions have been reported.

Bowers and Mims (1959) and Valishev (1965) have reported measurements of nickel ions diluted in zinc fluosilicate. The results did not agree with the predlctions of the usual theories of spin-lattice relaxation. Indeed, Valishev's results seem to be somewhat anomalous in that the relaxation time was observed to decrease with decreasing concentrations of nickel ions. However, this may possibly be explained by a phonon bottleneck. Lewis and Stoneham (1967) and Jones and Lewis (1967) report measurements for nickel in magnesium oxide. The relaxation times were explained in terms of a direct process at low temperatures and a Raman process at higher temperatures. However, the measured relaxation times were shorter than would be predicted from the relaxation theories. 


\section{B. Experimental Methods}

\section{Samples}

The crystals of lanthanum magnesium nitrate hydrate $\left(\mathrm{La}_{2} \mathrm{Mg}_{3}\left(\mathrm{NO}_{3}\right)_{12}\right.$ $24 \mathrm{H}_{2} \mathrm{O}$ ) doped with $\mathrm{Ni}^{2+}$ were supplied by $\mathrm{Mr}$. Akira Jindo. These crystals were grown by slow evaporation from a solution containing the correct proportions of $\mathrm{La}\left(\mathrm{NO}_{3}\right)_{3}$ and $\mathrm{Mg}\left(\mathrm{NO}_{3}\right)_{2}$ and a small amount of $\mathrm{Ni}\left(\mathrm{NO}_{3}\right)_{2}$. The crystals which are of rhombohedral symmetry, grow in the form of hexagonal plates with the crystal $c$ axis located perpendicular to the plane of the plate. The nickel lons are substitutional impurities for the magnesium ions. There are two kinds of magnesium sites which are called site 1 and site 2. (Zalk1n, et al., 1963). The spectra studied were due to nickel ions of trigonal symetry in site $l$.

Relaxation measurements were performed on two crystals one of which was more dilute in nickel lons than the other. The exact concentration of the nickel ions in the crystals is not known, but is not expected to exceed a maximum of $4 \%$. The concentration of the more concentrated sample is expected to be $1-2 \%$. The other crystal, which is known to be more dilute from a comparison of the relative spectrum intensities, is expected to have a concentration less than $1 \%$.

\section{Apparatus}

The crystals to be studied were held in a cylindrical cavity operating in the $\mathrm{TE}_{\mathrm{Oll}}$ mode. The crystals were oriented with the cylindrical axis of the cavity contained in the plane of the crystal so that the magnetic field could be rotated in the ac plane of the crystal. The relaxation time determinations were performed with the magnetic field oriented along the 
c axts which wss located with the following procedure. The magnetic field was rotated unt1l the two high field lines in the spectrum were superimposed. This occurred at the so-called magic angle of $37^{\circ}$ (Jindo, 1971) where the three nickel energy levels are accidentally equidistant. The magnet was then rotated by $37^{\circ}$ to obtain the $c$ axis.

The temperature of the sample was varied by changing the speed of pumping on the liquid helium in which the sample was immersed. The temperature, which was varied between 1.2 and $4.2^{\circ} \mathrm{K}$, was determined from the equilibrium vapor pressure of the helium.

The pulse-saturation spectrometer used in these experiments consisted of the normal homodyne detection spectrometer, which has been described previously (Pratt, 1967; Batchelder, 1969), and in addition, a PIN modulator, a pulse geverator, an oscilloscope, and a LAB-8/I digital computer system (see Fig. 35). The homodyne spectrometer was modified by the introduction of the Hewlett-Packard Model 8735B PIN modulator into the cavity arm of the bridge. This unit could vary the power reaching the cavity from full power to $80 \cdot \mathrm{dB}$ attenuation. In practice, the modulator and the attenuator in the cavity arm were adjusted so that the saturating power reaching the cavity : was about 50 milliwatts, while the observing power was more than $40 \mathrm{~dB}$ below the saturating power.

The modulator was pulsed to the full transmitting state by means of an Intercontinental. Instruments, Inc., Model PG-2 puise generator. The pulse widths were adjusted to about 5 milliseconds. Pulses were repeated at a maximum rate of once every $300 \mathrm{msec}$. which was sufficient to permit the spin system to return to equilibrium. The pulses were applied to the modulator through a biasing network which is also detailed in Fig. 35 . 


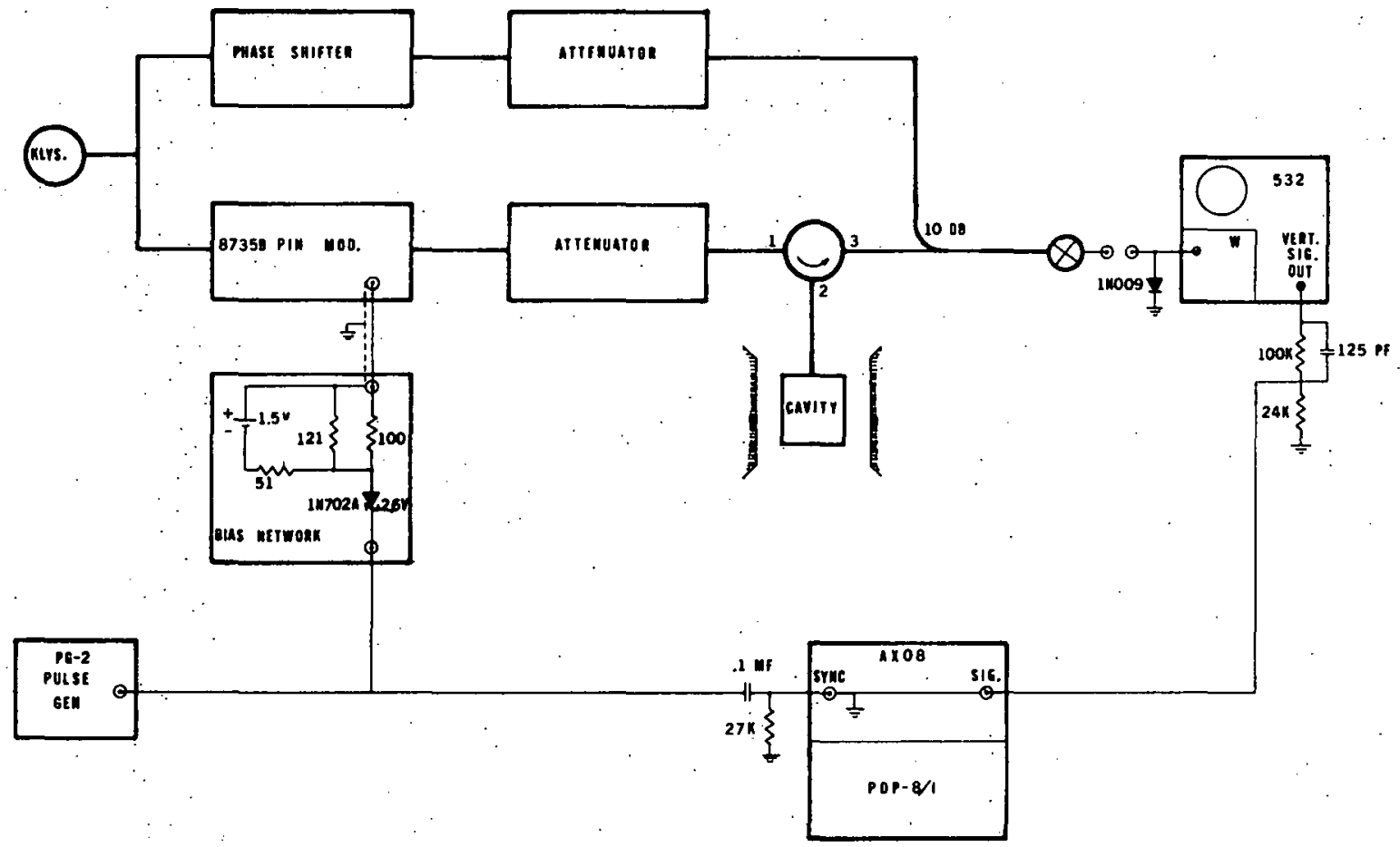

XBL 711-96

Fig. 35. Block diagram of pulse-saturation spectrometer. 
The biasing network served the double purpose of biasing the modulator to control the observing power, and to 1solate the pulse generator from the modulator. The pulse generator was found to have ripple in the output in its quiescent state which could modulate the observing power and cause noise. The zener diode in the network isolates the pulse generator after the pulse has been delivered and also prevents the pulse height from accidentally becoming large enough to damage the modulator. The biasing voltage was provided by a $1.5 \mathrm{~V}$ dry cell which is a staule DC vollage source. The resistors were adjusted for $c a: 45 \mathrm{~dB}$ attenuation in the modulator.

The recovery signals were observed as absorption signals using straight.DC detection from the crystal diode detector. The absorption signals were on the order of millivolts and could easily be displayed on an $x-y$ recorder or on an oscilloscope. The recovery curves were monitored w1th a Tektronix Model 532 oscilloscope equipped with a Type $W$ differential amplifier plug-in. The differential amplifier was necessary to offset the detector bias voltage in order to preserve the full gain in the amplifier. The type $W$ unit is susceptible to a thermal recovery problem if presented with a high signal level as would happen when a pulse was received. This effect was reduced by placing a clipping diode (1N009) on the input of the type $W$ unit to prevent overdriving. The oscilloscope also served the purpose of amplifying the recovery signals for presentation to the computer system.

A synchronizing signal from the pulse generator and the recovery signal from the oscilloscope vertical signal output were presented through impedance matching networks to the LAB-8/I digital computer system. 
The $L A B-8 / I$ computer system, as purchased from Digital Equipment Corp. consists of only a $4 \mathrm{~K}$ PDP-8/I computer with teletype, and an AX08 laboratory peripheral. Our system includes, in addition, a DF32 disk ( $32 \mathrm{~K}$ words), a PCO8 high speed paper tape reader and punch, a KE8I extended arithmetic element, and an extra $4 \mathrm{~K}$ of memory. The AX08 laboratory perlpheral processes all analog signals and contains an analog-to-digital converter $(A D C)$ which is multiplexed between four inputs, a crystal clock and an RC timing clock, a display control consisting of two analog outputs from a digital-to-analog converter and an intensify signal, and some trigger inputs for synchronization. There are also a number of miscellaneous digital inputs and outputs. The ADC is a 9 bit successive approximation converter and can perform a conversion in 17 microseconds. This is the ultimate limitation of how short a relaxation time may be measured with the computer system. The preamplifier of the ADC has a IIV input range.

The operation of the spectrometer is relatively straight-forward. The spectrometer is first tuned and adjusted in the normal manner, except that the klystron is not locked to the cavity. The klystron frequency lock is not used in order to prevent the lock recovery after a pulse from interfering with the signal recovery. The PIN modulator is adjusted to provide the proper attenuation for the observing power. The magnetic field is then adjusted to the proper resonance, and the pulse generator is turned on. The averaging program is then started (presently this is the BASIC AVERAGER provided by DEC). After a sufficient numer of scans have been accumulated, the averaging program is halted. At this time the LOGLINE overlay (see Appendix) is started to either punch the data on the 
high speed punch, or to analyze the data for relaxation times. The data are normally punched either for later analysis or to save the data.

\section{Rate Equations}

In a two level system relaxation can be viewed simply as a process of transferring spin population from one level to the othcr level. This process is readily described by a single decaying exponential. In a three level system the problem is somewhat more complicated. The population in one level may transfer to either of the two remaining levels. The signal observed in a relaxation experiment is proportional to the population difference between the two levels being observed, We must consider the form of the signal expected in a pulse saturation experiment. This is normally. done by means of the rate equations.

We shall consider the rate equations for the 3 level system shown in Fig. 36. Each of the levels, i, contains an instantanenus population $N_{i}^{O}$, and the excess pupulation, $n_{i}$, by

$$
N_{i}=N_{i}^{O}+n_{i}
$$

The levels are connected by the spontaneous transition probulilities

$w_{i j}$ (probability it j). The rate equations are then

$$
\begin{aligned}
& \dot{\mathrm{N}}_{1}=\mathrm{N}_{3} w_{31}+\mathrm{N}_{2} \mathrm{w}_{21}-\left(\mathrm{w}_{12}+\mathrm{w}_{13}\right) \mathrm{N}_{1} \\
& \dot{\mathrm{N}}_{2}=\mathrm{N}_{3} \mathrm{w}_{32}+\mathrm{N}_{1} \mathrm{w}_{12}-\left(\mathrm{w}_{21}-w_{32}\right) \mathrm{N}_{2}
\end{aligned}
$$

where the third equation for $\dot{\mathrm{N}}_{3}$ has been omitted since it is related to the other two by

$$
\dot{\mathrm{N}}_{1}+\dot{\mathrm{N}}_{2}+\dot{\mathrm{N}}_{3}=0
$$




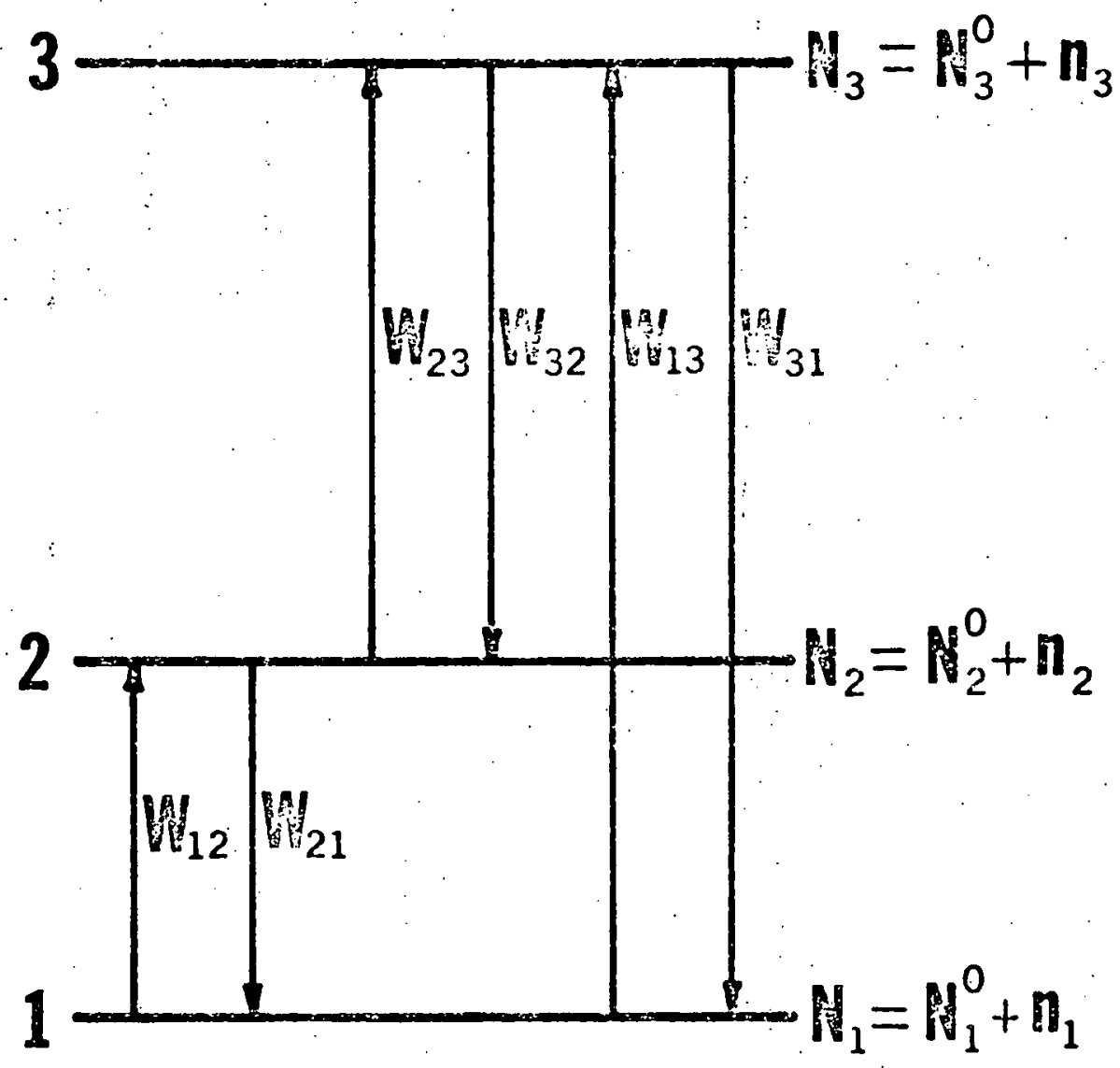

XBL $711-98$

Fig. 36. Transitions and populations of a three level system. 
However, since

$$
n_{3}=-n_{1}-n_{2}
$$

and

$$
\begin{aligned}
\mathrm{N}_{1}^{\circ} / \mathrm{N}_{2}^{\circ} & =\exp \left(-\mathrm{E}_{12} / \mathrm{kT}\right) \\
\mathrm{w}_{12} / \mathrm{w}_{21} & =\exp \left(\mathrm{E}_{12} / \mathrm{kT}\right)
\end{aligned}
$$

where $E_{12}$ is the energy difference between levels 1 and 2, and with similar. relations fur the other pairs of levels we may obtain the following equations

$$
\begin{aligned}
& \dot{n}_{1}=-\left(w_{12}+w_{13}+w_{31}\right) n_{1}+\left(w_{21}-w_{31}\right) n_{2} \\
& \dot{n}_{2}=\left(w_{12}-w_{32}\right) n_{1}-\left(w_{21}+w_{23}+w_{32}\right) n_{2}
\end{aligned}
$$

These are simultaneous linear differential equations of the form

$$
\begin{aligned}
& \dot{n}_{1}=a_{1} n_{1}+b_{1} n_{2} \\
& \dot{n}_{2}=a_{2} n_{1}+b_{2} n_{2}
\end{aligned}
$$

where

$$
\begin{aligned}
& a_{1}=-\left(w_{12}+\dot{w}_{13}+w_{31}\right) \\
& a_{2}=w_{12}-w_{32} \\
& b_{1}=w_{21}-w_{31} \\
& b_{2}=\left(w_{21}+w_{23}+w_{32}\right)
\end{aligned}
$$

These equations may be solved ustng standard techniques (Kâulull, 1958). The solutions are of the form

$$
n_{1}=\alpha \exp (\lambda t) \quad n_{2}=\beta \exp (\lambda t)
$$

where $\lambda$ may be obtained from the related homogeneous equations nbtained from $E q .5 .9$ and 5.7

$$
\begin{aligned}
& \left(a_{1}-\lambda\right) \alpha+b_{1} \beta=0 \\
& a_{2} \alpha+\left(b_{2}-\lambda\right) \beta=0
\end{aligned}
$$


These equations have a solution is the determinant is zero so that we may obtain

$$
\lambda=1 / 2\left(\left(a_{1}+b_{2}\right) \pm \sqrt{\left(a_{1}+b_{2}\right)^{2}-4\left(a_{1} b_{2}-a_{2} b_{1}\right)}\right)
$$

Ratios of alpha and beta may be obtained from Eq.s 5.10 and 5.11. In any case, the general solutions are

$$
\begin{aligned}
& n_{1}=c_{1} \alpha_{1} e^{\lambda_{1} t}+c_{2} \alpha_{2} e^{\lambda_{2} t} \\
& n_{2}=c_{1} \cdot \beta_{1} e^{\lambda_{1} t}+c_{2} \beta_{2} e^{\lambda_{2} t}
\end{aligned}
$$

where the C's are determined by the initial conditions. The solutions in which we are interested is the population difference given by

$$
n_{2}-n_{1}=c_{1}\left(\beta_{1}-\alpha_{1}\right) e^{\lambda_{1} t}+c_{2}\left(\beta_{2}-\alpha_{2}\right) e^{\lambda 2^{t}}
$$

Similar solutions may be obtained for the other level pairs. We can see that the observed recovery curve will be a sum of two exponentials rather than a simple single exponential. The relaxation times will be the reciprocals of the $\lambda^{\prime} \mathrm{s}$, and are related in a complicated manner by Eas. 5.11 and 5.8 to the transition probabilities. Theoretical results will, in general, be for the transition probabilities. Hence, a complete comparison of experiment with theory requires experimental values for the $w^{\prime} s$. This will necessitate a detailed consideration of the coefficients of the exponentials as well as the $\lambda$ :s. This will not be considered here since we have shown what we wished: the recovery curve is a sum of two exponentials. Furthermore, for a constant field, the same relaxation times should be obtained for each of the transitions. 


\section{Discussion and Results}

With the magnetic field oriented along the c axis of the crystal, the EPR spectrum of the Ni/LMN system consists of three lines which correspond to the transitions indicated in Fig. 37. The first and third lines, if the lines are numbered from low to high field, are strong allowed transitions. The second line is a "forbidden" transition. Jindo (1971) determined the spin Hamiltonian parameters to be $g_{\|}=2.235, g_{1}=2.233$, $D=+.2005 \mathrm{~cm}^{-1}$, and $E=.0007 \mathrm{~cm}^{-1}$. The small $E$ value was necessary to fit the data, though the ion with no lattice distortion is expected to be at a site of trigonal symmetry. These parameters have a small temperature dependence which may require minor modifications to any theoretical relaxation treatment of the system.

Relaxation time measurements have been made for each of the lines over a temperature range of 1.3 to $4.2^{\circ} \mathrm{K}$ using the pul se saturationrecovery method. An example of a typical recovery curve is shown in Fig. 38 which shows the recovery of the high field. Ine for $\mathrm{T}=1.37^{\circ} \mathrm{K}$. The data show an excellent signal to noise ratio and have been collected to essentially complete recovery so that the baseline of the recovery is well known. A logarithmic presentation of the data with the baseline substracted is shown in Fig. 39. The logarithm is essentially linear at long times but showg curvature near the start of the recovery curve. Possible reasons for this behavior are discussed later. A reasonable relaxation time may be obtained from the linear portion of the curve.

The relaxation data obtained for the low and high field lines of the falrly concentrated sample are presented in Figs. 40 and 4I. The data are 


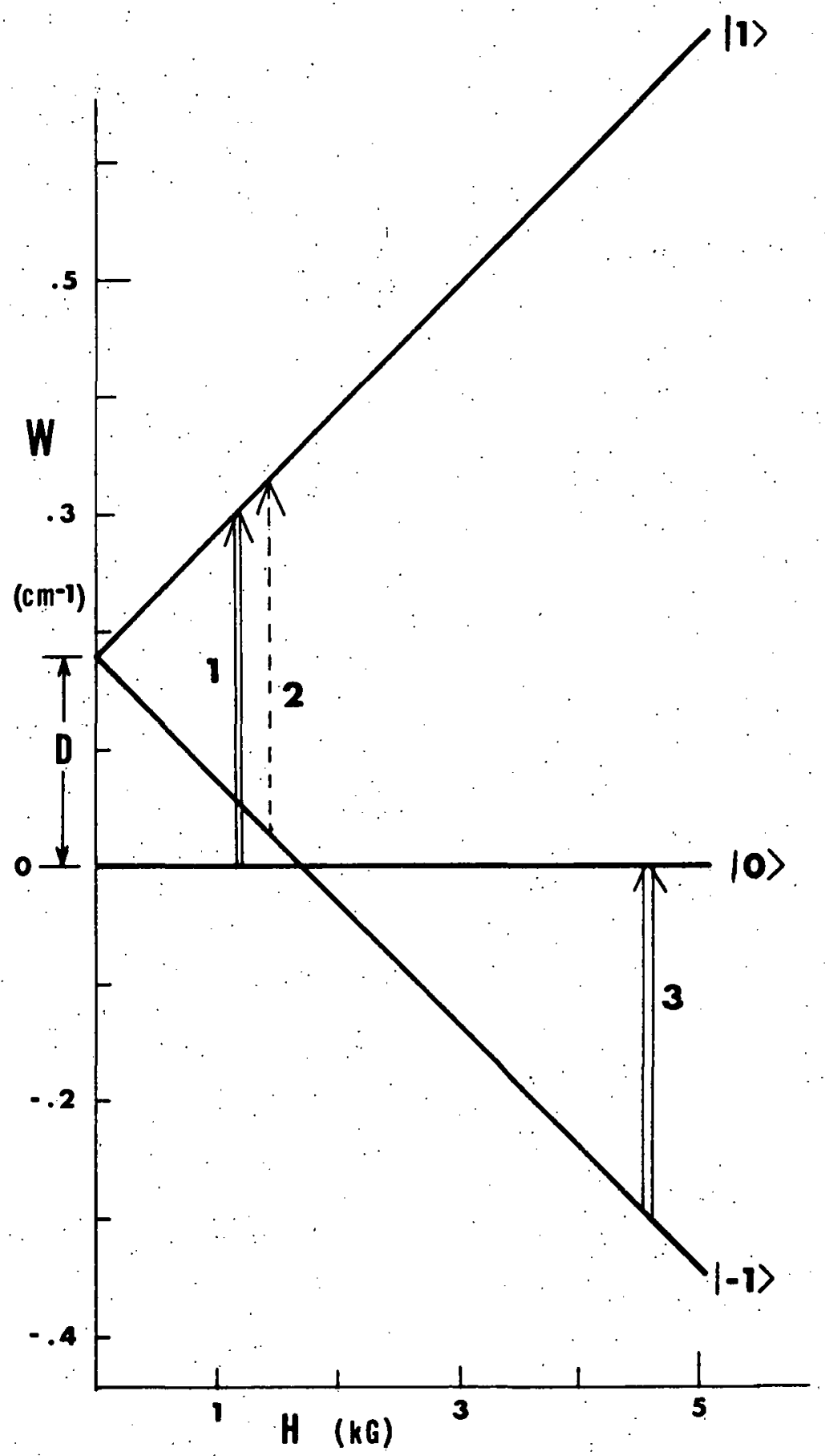

XBL $711-100$

Fig. 37. Energy levels and transitions for $\mathrm{Ni}^{2+}$ in lanthanum magnesium nitrate hydrate with the magnetic field oriented along the $c$ axis (Jindo, 1971) 


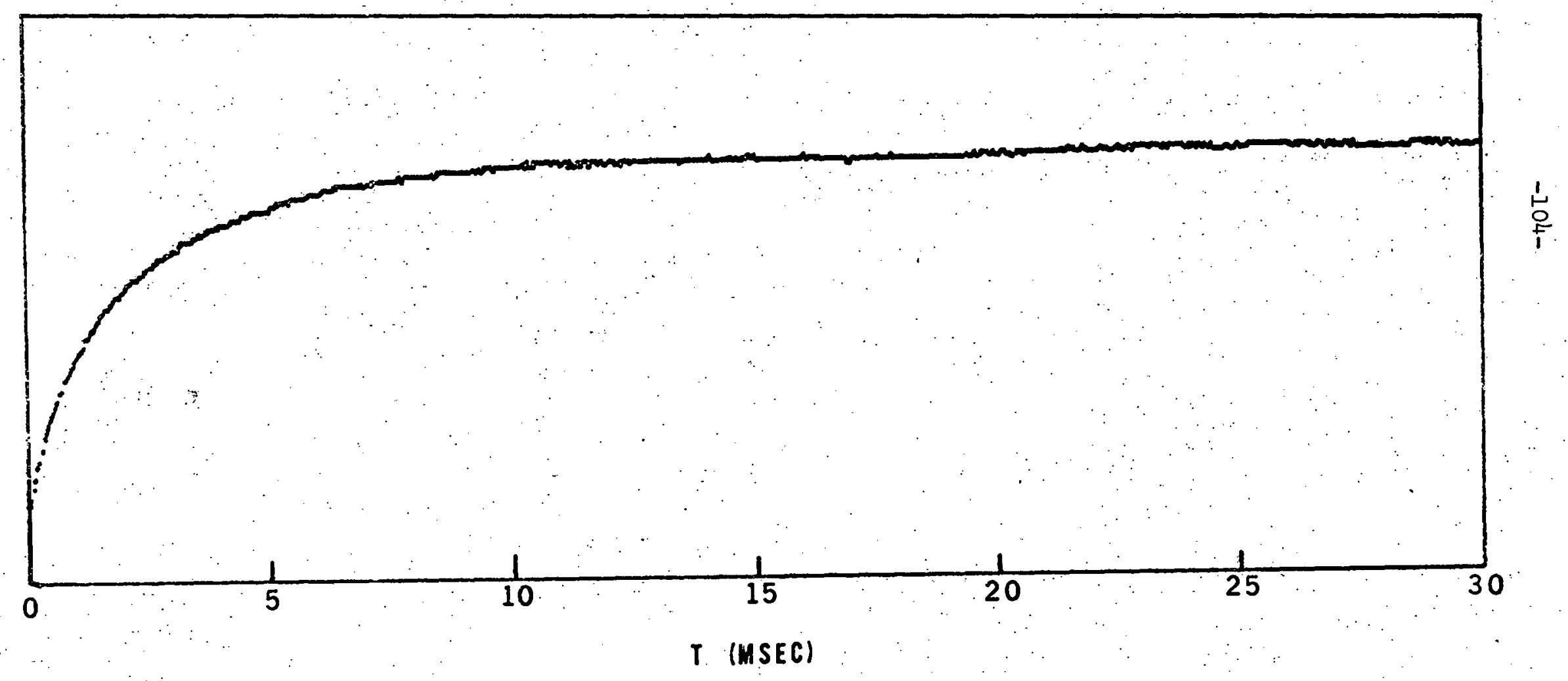

Fig. 38. Exporeztial recovery curre Eor the low field line at $1.37^{\circ} \mathrm{K}$. An average of 50 experiments. 


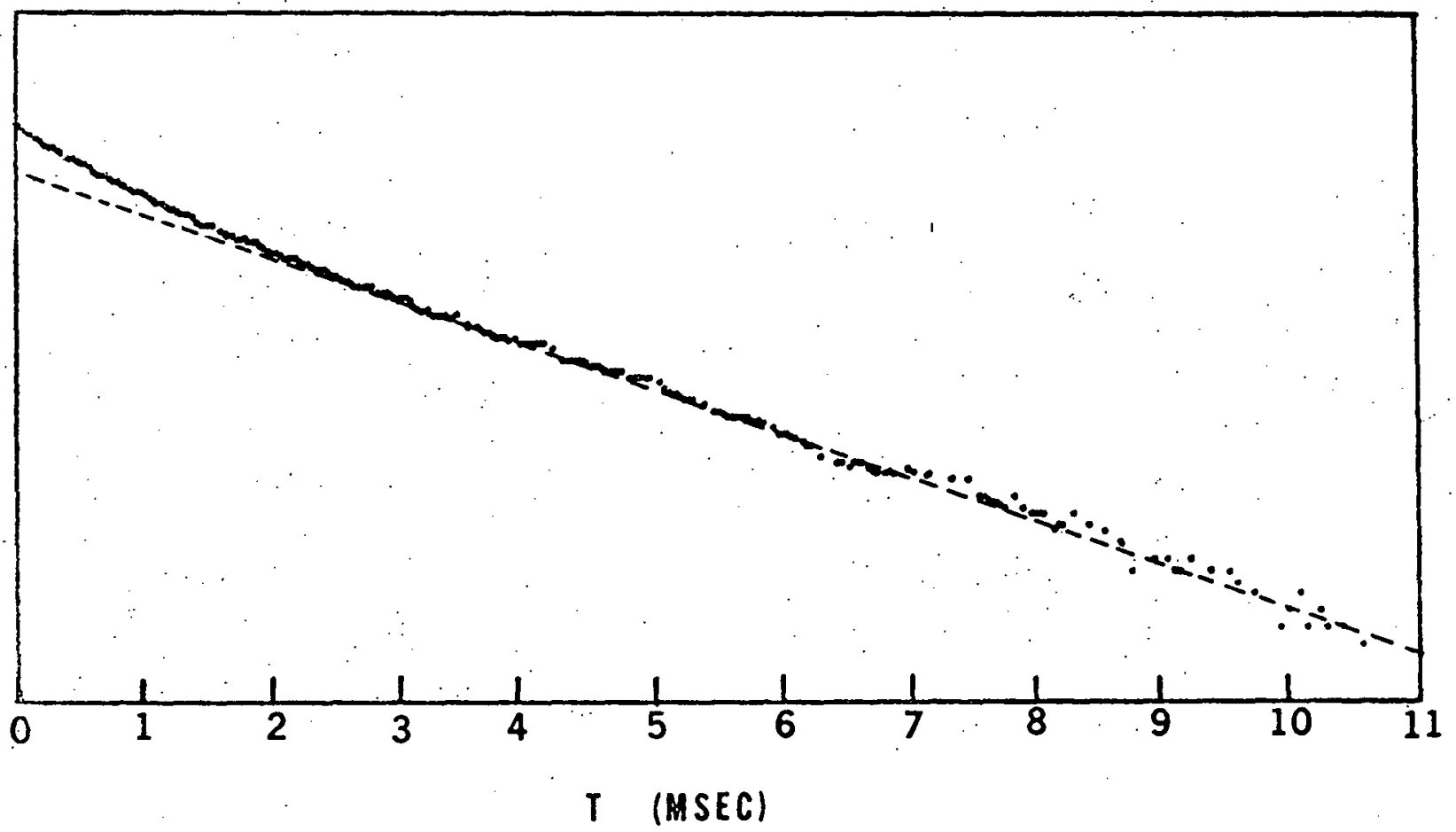

Fig. 39. Logarithm of the data in Fig. 38 with the baseline removed. Dashed line is a straight line fit to the data with a time constant (reciprocal slope) of $3.15 \mathrm{msec}$. Curvature at start of data may indicate the presence of a second shorter time constant. 
$\cdot-106-$

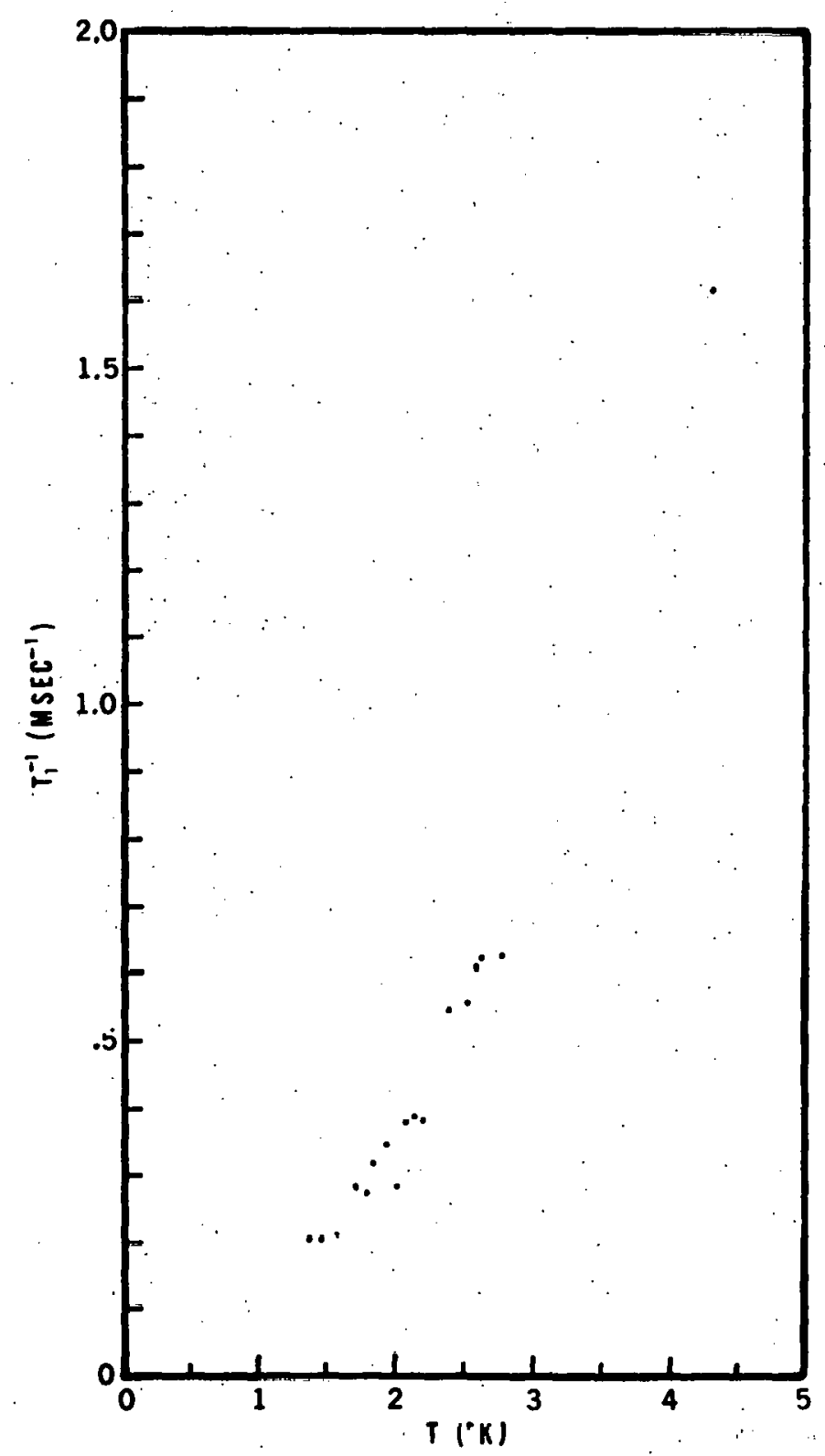

XBL 711-9y

Fig. 40. $T_{1}^{-1}$ vs: $T$ for the low field line. 


$$
-107-
$$

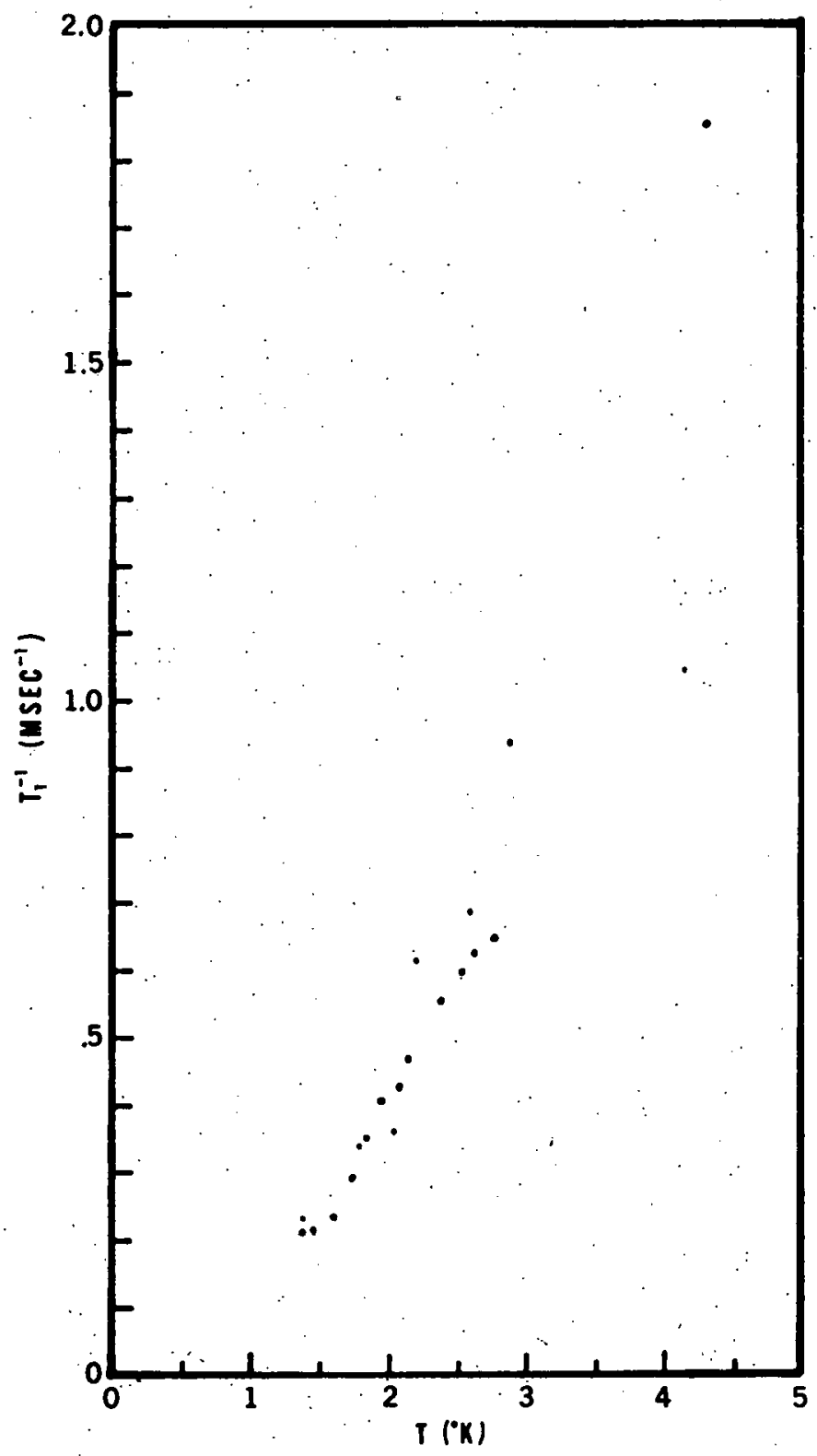

$X B L 711-97$

Hg. 41. $\mathrm{T}_{1}^{-I}$ vs. $T$ for the high field line. 
somewhat scattered so that a functional relationship between $\mathrm{T}_{1}^{-1}$ and the absolute temperature may not be established with confidence. The lack of data between 3 and $4^{\circ} \mathrm{K}$ is also somewhat unfortunate. This data was not obtained in these preliminary experiments because of the difficulty of regulating and measuring the temperature with our present apparatus. The data suggest that the inverse relaxation time is linear in the absolute temperature, but the scatter is such that a quadratic could also fit the तata.

The data obtained for the dilute sample are similar to that for the concentrated sample. The data were more difficult to obtain since the signal was weaker than in the concentrated sample. The data seem to indicate a slightly shorter relaxation time in this sample, but this may in fact be experimental error.

Relaxation times were also determined for line 2, the "forbidden" line in the spectrum. The intensity of this transition was very much weaker than that of the other two transitions and the data were correspondingly more difficult to obtain. The relaxation times for this line were much longer than those for the other two lines, but this result is doubtful at best. In fact, the relaxation times varied quite widely and may be an experimental artifact.

A number of relaxation mechanisms have been utilized to explain the data in solid state measurements (Standley and Vaughan, 1969). For a nonKrailiers sull, such as $\mathrm{N1}^{2+}$ system, the direct process, the Kaman process, and the Orbach process give rise to a linear, a seventh power, and an exponential temperature dependence, respectively, for the inverse relaxation times. The data in the $\mathrm{Ni} / \mathrm{LMN}$ system could be consistent with either 
a linear or a quadratic function of temperature. The linear function would be consistent with a direct process. However, if a straight line is fit to the data and extrapolated to zero temperature, the line does not pass through zero, whereas the contribution from the direct process to $\mathrm{T}_{1}{ }^{-1}$ should go to zero.

The data would seem to be fit better with a quadratic function of the form

$$
\mathrm{T}_{1}^{-1}=\mathrm{D} \mathrm{\textrm {T } ^ { 2 }}
$$

where $\mathrm{D}$ is a proportionality constant: This temperature dependence is consistent with a phonon bottleneck wherein a phonon-bath interaction rather than a spin-lattice process is observed. If we attempt to least squares fit the data with Eq. 5.14 , we obtain a value of $D=.0874$ for the low field line (Fig. 40) and $D=.0866$ for the high field line (Fig. 4l). These values are essentially identical. If the observed relaxation is due to a Phonon bottleneck process, we would expect $D$ to be directly proportional to the resonance linewidth and inversely proportional to the concentration and the thickness of the crystal (Scott and Jeffries, 1962). The possibility of: a shorter relaxation time in the dilute sample supports the proposal of a phonon bottleneck. The relaxation time is not drastically shorter in the dilute sample because of compensation from the dependence on the linewidth: The linewidth for the dilute sample is much narrower than that of the concentrated sample. The relaxation time seems to be well described by a phonon bottleneck, although we cannot completely rule out the posssibility of a direct process since the data are scattered. 
It was mentioned earlier that the logarithm of the recovery curve exhibited curvature near the start of the recovery. This could indicate the presence of a second relaxation time in the recovery. Data was obtained for the short time constant by stripping the part of the curve due to the longer time from the data and then examining the residual recovery curve. However, the relaxation times obtained by this procedure were extremely scattered. No correlation with temperature or with the longer relaxation time was evident.

Part of the difficulty in these determinations lies in the analytical procedure. The second recovery curve is revealed by removing from the original data that pcrtion which is due to the first recovery curve. Small errors in the determination of the first recovery curve could cause large deviation in the second curve which is much smaller than the first curve. 'It should be mentioned that the second curve is itself a cause of error in determining the first curve.

The short relaxation time may be due to two main sources. First, if the relaxation is phonon bottlenecked, the short relaxation could be the tail end of a $T_{1}$ relaxation which is becoming rapidly bottlenecked. This relaxation might follow a direct process, but the determination is very difficult. Secondly, if the observed relaxation is the true spin-. lattice process, then two relaxation times are expected as shown in Section V-C. Furthermore, if the process is phonon bottlenecked but the short relaxation is the true relaxation time, then this short relaxation process. should contain both of the relaxation times predicted in section $\mathrm{V}-\mathrm{C}$. If this is so, the analysis of the short relaxation time is quite difficult. 
It is possible that other processes are responsible for the initial relaxation. The first problem is whether the spectrometer is linear in its response to the recovery signal. The signals which are observed are quite strong so that with relatively large changes in cavity $Q$, the spectrometer may be nonlinear. The amplifiers may be overdriven so that the short relaxation may be the end of the recovery of an overdriven system.

A second problem is that of spectral diffusion in the line. If the nickel lines are inhomogeneously broadened, then it is possible to saturate the center of the line, but then observe a recovery due to the wings of the line growing toward the center. This would give a hyperbolic recovery curve (Mims, et al., 1961). The short relaxation time could then be an incorrect analysis of the recovery curve.

It is evident that much work remains to be done in the $\mathrm{Ni} / \mathrm{LMN}$ system. Detailed studies of the relaxation times should be made for each of the transitions as a function of temperature. The crystal size and the nickel concentration should be varied to confirm the hypothesis of a phonon bottleneck. The concentration study is also important for studies of exchange effects. Studies should also be made of the dependence of the relaxation times on the pulse widths and on the value of the saturating power. (Preliminary investigations indicated no dependence). Possible anisotropy in the relaxation times should be studied by making measurements as a function of angle. Indeed, measurements at the so-called magic angle of $37^{\circ}$ ( $\mathrm{Jindo}, 1971$ ) could prove very interesting. At this angle the nickel transitions are superimposed, since the energy levels are accidentally equidistant. The possibility of double quantum transitions exists, especially at high power levels. A double quantum transition should 
not occur at the power levels used for monitoring. However, the initial spin distributions produced by the saturating pulse might be studied. Furthermore, Slichter (Slichter, 1963; Hebel and Slichter, 1959) has shown that, in systems with equidistant energy levels whi.h can be described by a spin temperature, orly one relaxation time should be observed.

In summary, we have developed an experimental method which should be useful for spin-lattice relaxation time measurements. The acquisition and aralysis process in our small computer system should be relatively corvenient. However, the problems associated with the operation of a pulse spectrometer are still present, and they are the reason for the scatter in our data. Possible problems are the drift of the klystron frequency which would cause drifts in the recovery signal, and possible interference effects from the dispersion signal. Since the relaxation time could vary across the reasonance lineshape, the setting of the resonance magnetic fipld is a pnooible errur youret. 'l'hese problems should be studied and eliminated. 


\section{APPENDIX I: LORENTZ LINESHAPES AND \\ LEAST SQUARES THEORY}

The real and imaginary parts of the complex susceptibility, $X=\chi^{\prime}+i \chi^{\prime \prime}$, which are derived from the Bloch Equations are: given

$$
\begin{aligned}
& X^{\prime}(\omega)=I / 2 x_{0} \omega_{0} \cdot \frac{\mathrm{T}_{2}^{2}\left(\omega_{0}-\omega\right)}{1+\mathrm{T}_{2}^{2}\left(\omega_{0}-\omega\right)^{2}+\gamma^{2} \mathrm{H}_{1}^{2} \mathrm{~T}_{1} \mathrm{~T}_{2}} \\
& X^{\prime}(\omega)=1 / 2 x_{0} \omega_{0} \frac{\mathrm{T}_{2}}{1+\mathrm{T}_{2}^{2}\left(\omega_{0}-\omega\right)^{2}+\gamma^{2} \mathrm{H}_{1}{ }^{2} \mathrm{~T}_{1} \mathrm{~T}_{2}}
\end{aligned}
$$

where $H_{1}$ is the rf magnetic field, $T_{1}$ and $T_{2}$, the spin-lattice and spinspin relaxation times respectively, $X_{0}$ the static magnetic susceptibility, and $\gamma$ the magnetogyric ratio relating the resonance frequency, $\omega_{0}$, to the resonance field, $\mathrm{H}_{0}$, by $\omega_{0}=\gamma \mathrm{H}_{0}$. In the typical magnetic resonance experiment the spectrometer is tuned to observe only the absorption, $\chi^{\prime \prime}$ so that the dispersion, $X^{\prime}$, is neglected. Furthermore, magnetic resonance experiments are performed at low powers so that $\gamma^{2} \mathrm{H}_{1}{ }^{2} \mathrm{~T}_{1} \mathrm{~T}_{2} \ll I$ and $\chi^{\prime \prime}$ reduces to

$$
x^{\prime \prime}(\omega)=1 / 2 x_{0} \omega_{0} \frac{T_{2}}{1+T_{2}^{2}\left(\omega_{0}-\omega\right)^{2}}
$$

Examination of Eq. A-3 reveals that it is similar to the Lorentz lineshape function

$$
f(\omega)=\frac{T_{2}}{\pi} \frac{1}{\left.\left.1+T_{2}^{2}(1)-(1)\right)_{0}\right)^{2}}
$$

Hence, the susceptibility is usualy written in the form of a Lorentz function

$$
-X^{\prime \prime}(\omega)=F \frac{\ddot{T}_{2}}{\pi} \frac{1}{I+T_{2}^{2}\left(\omega-\omega_{0}\right)^{2}}
$$

where $F$ is the line intensity such that.

$$
F=\int_{-\infty}^{\infty} X^{\prime \prime}(\omega) d \omega .
$$


From Eq. A-4 we can see that the maximum in the function is $T_{2} / \pi$ and also that $\mathrm{T}_{2}$ is the half width at half height.: We may also observe that the units of $\mathrm{T}_{2}$ are seconds per radian.

Because of experimental convenience magnetic resonance experiments are performed with the magnetic field as the independent variable rather than the frequency so that the Ineshape becomes

$$
L(H)=\frac{F}{\pi} \cdot \frac{\delta}{\delta^{2}+\left(H-H^{0}\right)^{2}}
$$

where $\delta$ is the half width at half height (in gauss), and $H^{\circ}$ is the resonance center of the line. Furthermore, EPR spectroscopy is done with the derivative of the absorption given by

$$
\begin{aligned}
L^{\prime} \cdot(H) & =-\frac{2}{\pi} F \delta \frac{H-H^{\circ}}{\left[\delta^{2}+\left(H-H^{\circ}\right)^{2}\right]^{2}} \\
& -\frac{2}{\pi} \text { F } \delta \frac{H^{\circ}-H}{\left[\delta^{2}+\left(H^{\circ}-H\right)^{2}\right]^{2}}
\end{aligned}
$$

The peak-to-peak distance, $\Delta \mathrm{H}$, of this function is given by

$$
\Delta \mathrm{H}=(2 / \sqrt{3}) \delta
$$

and is related to $\mathrm{T}_{2}$ by Eq. 1.2 .

In general an EPR spectmum consists of a number of lines with different centers, widths, and intensities, so that the spectrum is described by a sum of Lorentz lineshapes

$$
g(H)=\frac{2}{\pi} \sum_{k} F_{k} \frac{\left(H_{k}^{O}-H\right) \xi_{k}}{\left[\left(H_{k}^{O}-H\right)^{2}+\delta_{k}^{2}\right]^{2}}
$$

Except in unusual cases the $\mathrm{H}_{\mathrm{k}}^{\circ}$ are not independent of each other. A spectrum with several components is usually due to hyperfine structure so that the line centers are determined by a spin Hamiltonian. The line 
centers ars then given to second order by (for spin $1 / 2$ complexes)

$$
y_{k}^{u}=H_{0}-A m-A^{2}\left(\left(I(I+I)-m^{2}\right) / 2 H_{0}\right.
$$

where $\mathrm{H}_{\mathrm{O}}$ is the spectrum center, $A$, the hyperfine coupling constant; I, the nuclear spin, and $m$, the nuclear spin quantum number. The $k^{\prime}$ th line corresponds to a given value of $\mathrm{m}$. In the general case the spectrum is describea by more than one coupling constant and $\mathrm{H}_{\mathrm{k}}^{\mathrm{O}}$ is given by

$$
H_{k}^{C}=H_{0}-\sum\left(A_{j} m_{j}+A_{j}{ }^{2}\left(\left(I_{j}\left(I_{j}+1\right)-m_{j}{ }^{2}\right) / 2 H_{0}\right.\right.
$$

In the case of organic radicals the second order contribution is usually neglected.

A further simplication is usually possible when the lines are due to hyperfine structure. In this case the line intensities are usually given by the ratios of whole numbers which can be computed from the number of ruclei and the nuclear spin. The line intensity is a known fraction of the total intensity of the overall spectrum. We may then rewrite the Iineshape as

$$
g(H)=\frac{2}{\pi} F \sum_{k} R_{k} \frac{\left(H_{k}^{0}-H\right) \delta_{k}}{\left[\left(H_{k}^{0}-H\right)^{2}+\delta_{k}^{2}\right]^{2}}+S+D H
$$

where $K_{k}$ is the intensity ratio for the $k^{\prime}$ th hyperfine component. $S$ and $D$ are terms which are added to account for a baseline shift and a baseIine drift. In the most general case the spectrum consists of several species each of which has a lineshape characterized by Eq. A-12. The lineshape then consists of a sum of terms similar to the first term in Eq. $1-12$.

We are now in possession of an analytic function to describe our experimental spectra. If we have values for the spectrum intensities at 
various magnetic fields, we can perform a least squares fit of the function in Eq.A-12. The theory of least squares is well known and will not be rederived here (Deming, 1938; Shoemaker and Garland, 1962). However, the object is to minimize the sum of the squares of the differences between the experimental points $y(H)$ and the theoretical value $g(H)$. For this purpose we require the values of the derivatives of Eq. A-12 with respect to each of the parameters which we wish to determine. These derivatives are given by

$$
\begin{aligned}
& \frac{\partial g(H)}{\partial F}=\frac{2}{\pi} \sum_{k} R_{k} \frac{\left(H_{k}^{O}-H\right) \delta_{k}}{\left[\left(H_{k}^{\circ}-H\right)^{2} \delta_{k}^{2}\right]^{2}} \\
& \frac{\partial g(H)}{\partial \delta_{k}}=\frac{2}{\pi} \quad F \quad R_{k} \frac{\left(H_{k}^{\circ}-H\right)^{3}-3\left(H_{k}^{\circ}-H\right) \delta_{k}^{2}}{\left[\left(H_{k}^{\circ}-H\right)^{2}+\delta_{k}^{2}\right]^{3}} \\
& \frac{\partial g(H)}{\partial H_{0}}=\frac{2}{\pi} \quad F \sum_{k}(1+f(k)) \frac{\delta_{k}^{3}-3\left(H_{k}^{0}-H\right)^{2} \delta_{k}}{\left[\delta^{2}+\left(H_{k}^{0}-H\right)^{2}\right]^{3}} \\
& f(k)=\sum_{j} \frac{A_{j}{ }^{2}}{2 H_{0}^{2}}\left[I_{j}\left(I_{j}+I\right)-m_{j}^{2}\right] \\
& \frac{\partial g(H)}{\partial A_{i}}=-\frac{2}{\pi} F \sum_{k}\left[m_{j}+\frac{A_{j}}{H_{0}}\left(I_{j}\left(I_{j}+I\right)-m_{j}{ }^{2}\right)\right] \\
& x R_{k} \frac{\delta_{k}^{3}-3\left(H_{k}^{0}-H\right)^{3} \delta_{k}}{\left[\delta_{k}^{2}+\left(H_{k}^{0}-H\right)^{2}\right]^{3}} \\
& \frac{\partial g(H)}{\partial S}=I \\
& \frac{\partial g(H)}{\partial D}=H
\end{aligned}
$$


The value of $\mathrm{k}$ is determined by a particular combination of $\mathrm{m}_{\mathrm{f}}$ values. The value of the function, $f(k)$ is different for each $k$ since the values of $\mathrm{m}_{\mathrm{f}}$ change. In the case of transition metal complexes with hyperfine only from the central ion; the spin Hamiltonian is given by Eq. A-10 rather than Eq. A-ll so that the sum over $\mathrm{j}$ reduces to one term. Using a standard least squares treatment the derivatives are formulated into a set of normal equations in the following manner.

Let us assume that the parameters which we seek are $x_{n}$. We have an inftial: guess $x_{n}^{0}$ and we wish to find a value $\Delta x_{n}$ to obtain a new value for the parameters

$$
x_{n}=x_{n}^{0}+\Delta x_{n}
$$

We also assume that the errors are only in the $\mathrm{y}(\mathrm{H})$ with no errors in $\mathrm{H}$. Then we must solve the following set of equations

$\sum_{i} \frac{\partial g_{i}}{\partial x_{1}} \frac{\partial g_{i}}{\partial x_{1}} \Delta x_{1}+\sum \frac{\partial g_{i}}{\partial x_{1}} \frac{\partial g_{i}}{\partial x_{2}} \Delta x_{2}+\ldots+\sum_{i} \frac{\partial g_{i}}{\partial x_{1}} \frac{\partial g_{i}}{\partial x_{n}} \Delta x_{n}=\sum \frac{\partial g_{i}}{\partial x_{1}}\left(y_{i}-g_{i}\right)$

$\sum_{i} \frac{\partial g_{i}}{\partial x_{2}} \frac{\partial g_{i}}{\partial x_{1}} \cdot \Delta x_{1}+\sum_{i} \frac{\partial g_{i}}{\partial x_{2}} \frac{\partial g_{i}}{\partial x_{2}} \Delta x_{2}+\ldots+\sum_{i} \frac{\partial g_{i}}{\partial x_{2}} \frac{\partial g_{i}}{\partial x_{n}} \Delta x_{n}=\sum_{i} \frac{\partial g_{i}}{\partial x_{2}}\left(y_{i}-g_{i}\right)$

$\sum_{i} \frac{\partial g_{i}}{\partial x_{n}} \frac{\partial g_{1}}{\partial x_{i}} \Delta x_{1}+\sum \frac{\partial g_{i}}{\partial x_{n}} \frac{\partial g_{i}}{\partial x_{2}} \Delta x_{2} \cdot+\ldots+\sum \frac{\partial g_{i}}{\partial x_{n}} \frac{\partial g_{i}}{\partial x_{n}} \Delta x_{n}=\sum \frac{\partial g_{i}}{\partial x_{n}}\left(y_{i}-g_{i}\right)$

The sums are taken over all the points in the spectrum. These equations are of the form

$$
\underline{A} \underline{X}=\underline{B}
$$

where $\underline{A}$ is an $N X N$ matrix where $N$ is the number of parameters to be determined, $X$ and $B$ are vectors. These equations are solved by standard methods, for example, matrix inversion, to obtain values for the corrections $\Delta x_{n}$. 
New values of the parameters are obtained from Eq. $A-18$. In the simplest case this is all that is necessary to obtain a least squares value for the parameters. In general we must perfrcm an iterative procedure, using the new value for the parameter as a starting point for another solution of Eq. A-19. The iterations are then repeated until the residuals are minimized in the least squares sense. If the fitted function is linear in the parameters, then the iterative procedure will converge rapidly to a solution. Unfortunately, the Lorentz function is not Iinear in the parameters.

A non-linear least squares treatment is not guaranteed to converge. In fact, the correction vectors obtained from the solution of Eq. A-19 may rot: be correct in magnitude. In this case the solution may diverge or may oscillate widely about the true solution. This behavior is minimized by adjusting the correction vectors so that only a fraction of the magnitude is used to compute the new value of the parameter. The fraction used will depend upon the parameter and the function. 


\section{APPENDIX II: DIGITAL PROGRAMS}

\section{A. Data Acquisition. System Programs}

\section{SUMTAP3}

The SUMTAP3 ${ }^{*}$ program is used to read the magnetic tapes which are produced by the data acquisition system (Model 56114 ) and to produce a Fortran compatible date tape for processing by FITESR (see following discussion): The program will read the data tape; select the data in a given file corresponding to either up-field or downfield sweeps, disregarding data inconsistent with smooth sweeps; smooth the $\mathrm{y}$ values with a second order polynomial; store the smoothed data points in predetermined channels; and sum complete sweeps to produce an averaged spectrum. The output from the program consists of a printed listing of the summed and normalized spectrum, a plot of the spectrum and its integral, and a magnetic tape of the spectral data for further processing by least squares programs.

\section{a. Format of Input Data Tape}

The data acquisition system organizes a special work mark character, the 7 digit counter measurement, and the 5 digit voltmeter measurement plus a sign into a 14 character word: A word is a measured data point. The words are organized into 80 word records and is recorded in IBM compatible NRZI format on the magnetric tape. The data belonging to a spectrum consists of a mmber of 80 word record blocks which make up a file. The word "file" is used with two slightly different meanings in this discussion. When applied to the input tape, the word, "fille", refers to a record or number of records which is isolated from other similar records by a file mark or end-of-file gap. The data within a file may *

Original written by Dr. Alfred Bader (Bauder and Myers, 1968). 
consist of one or more spectra. When applied to the output tape, the word, "file", refers to the data for a given spectrum. It is not separated from other such files by an end-of-file gap. (This permits the data to be read by a Fortran program without special tests for file marks.)

Both upfield and downfleld sweeps may be included in a single file on the input tape. If severul sweeps are included in a single f'ile, the sweeps are averaged to form an averaged spectrum on the output tape. However, only upfield sweeps or nnly downfield sweeps may be averagcd; upfield and downfield sweeps may not be averaged together. Two different spectra may be obtained from a mixiure of upfield and downfield sweeps, one the average of the upfield sweeps, and the other the average of the downfield sweeps. The xasxis data of given sweep must: be either all increasing or all decreasing. Otherwise, the sweep may be regarded as incomplete and ignored.

The end-of-file gap on the input tape serves the purpose of indicating to the program the end of a given data set. When the file gap is encountered; the program will plot the spectrum it has found within the file and also write the spectrum on the output tape in a. Fort.ran compatible format. If a file gap is encountered before data is found, the file is regarded as empty. Note that, therefore, empty files count as flles for the purpose of numbering the files. If four sequential file marks are encountered without data, the program will assume that the tape contains no more spectra and will terminate. For this reason, all tapes should be ended with four file marks to ensure program termination. 


\section{b. Input Data Cards}

The data cards are used to describe the spectra on the input tape and to indicate the processing to be performed on the spectra. Card I (Format 8A10)

NAME An arbitrary name for the output tape which will contain the smoothed spectra. 10 alphanumeric characters. This name is used to identify the tape for subsequent programs.

COMMENT any arbitrary title or comment to be printed for identification Card 2 (Format 3I5, 25X, 4AIO)

NFILEl the number of the file to be analyzed. Files must be processed in the order in which they are found on the tape. If several fites are to be processed (by providing several of card 2), the :files must be processed in order of increasing file number.

IK $\quad=+1$ upfield sweep $=-1$ downfield sweep

$\mathrm{KK}=0$ do not read a new card 3. Assume the parameters are the sam? as provided by a previous card 3 :

$\neq 0$ read new parameters from the card 3

Card 3 (Format 2I5, 2EiO.0) provided only if $K K \neq 0$

KP spectra will be smoothed with $2 * K P+I$ points ( $K P$ must be less than 10)

NUM number of smoothed data points of the spectrum to be stored on the output tape

FMIN lower limit for sweep

FMAX upper limit for sweep; must always be greater than FMIN (FMIN and FMAX are in the units of the $x$ axis which wes recorded on the input tape) 
The following cards repeat the sequence from card 2, until no further files are to be processed. A blank card at the end of the data deck (which corresponds to NFILEI=0) is used to terminate program execution.

c. Subroutines

गMOTHE

SMOO'IIH2 is used to perform digital filtering (Savitzky and Golay, 1964) of the data and to select the absorption value for a given channel. It is assumed that measurements of the frequency ( $x$ axis) and the derivative absorption signal ( $y$ axis) are made at equal intervals of time. Both of the measurements are subject to noise, which is to be minimized with a smoothing function. The $x$ axis is smoothed with a linear function $x=r+s * t$. The $y$ axis is smoothed with a quadratic, $y=a+b * t+c * t * * 2$. Here, $t$ is the relative time of the measurement.

The program uses $2 * \mathrm{KP}+1$ points and automatically selects those points which are evenly distributed on both sides of a channel. It uses the linear function to calculate $r$ and $s$ and then uses these constants to determine the time difference from the center of the current channel to the nearest experimental point. The y values are smoothed simultaneously with the quadratic to determine the constants, $a, b$, and $c$. A smoothed value for $y$ at the center of the channel is then corputed.

The determination of the constants $a, b$ and $c$, is simplified by the assumption of equal time intervals in the measurements. The time interval is assumed to be unity so that the relative time is an integer between $-K P$ and $+K P$. The constants are then determined by a least squares procedure by solving the following normal equations: ( $k=k P)$ 
a $\underset{-k}{\sum_{-k}^{k}} i+b(0)+c \sum_{-k}^{k} I^{2}=\sum_{-k}^{+k} y_{I}$

$a(0)+b \sum_{-k}^{k} I^{2}+c \cdot(0)=\sum_{-k}^{+k} I y_{I}$

$a \quad \underset{-k}{+k} I^{2}+b(0)+c \sum_{-k}^{k} I^{4} \cdot \sum_{-k}^{+k} I^{2} y_{I}$

The solution is simplified with the following summation formulae:

$$
\begin{aligned}
& \sum_{-k}^{+k} I=\sum_{-k}^{+k} I^{3}=0 \\
& \sum_{0}^{k} I^{2}=(1 / 6) k(k+1)(2 k+1)
\end{aligned}
$$$$
\sum_{0}^{k} I^{4}=(1 / 30) k(k+1)(2 k+1)\left(3 k^{2}+3 k-1\right)
$$

\section{IBITS $(I 1, I 2, X)$}

IBITS is an ASCENTF coded subroutine which selects the bits Il to $I 2$, inclusive, of $X$ and stores them right justified in IBITS." The bits are numbered from 1 to 60 from left to right. The spectral data on the input tape is read by SUMTAP3 as BCD coded information without conversion to a binary number. SUMTAP3 performs the BCD to binary conversion. The IBITS subroutine is used to locate a digit from the tape so that SUMTAP3 can perform the conversion to binary. This subroutine is coded specifically for a CDC 6600 digital computer, and may require changes if the computer or operating system is changed. 


\section{$-124-$}

\section{Plotting Subroutines}

The following subroutines are used to plot the spectra. They are assumed to be available in the computer library as part of the FORTRAN compiler. A brief description is included to illustrate their usage. CCGRID (5,2, 6HNOIBLS, 4). This subroutine draws a grid around the spectrum. The $x$ axis is divided into 5 divisions each of which is subdivided into two parts by tick marks. The y axis is divided into 4 divisions. The grid is not to be labeled with a scale. CCLTR (20., 300., 1,2) This subroutine letters the graph at $x$ position 20. and $y$ position 300 . In a direction specified by 1 and a size specified by 2 . The information to be lettered has been previously provided. CCPLOT (SX,SY,NUM, 4HJOIN, O,O) Plots the NUM points in (SX,SY). The first 0 specifles a symbol for the plot, and the second 0 indicates that every point in the array is to be plotted. CCNEXI' 'I'his subroutine spaces the chart paper so that another graph may be plotted. The previous graph is ended. CCEND. This subroutine is called at the conclusion of the program to terminate the plotting.

\section{Special Subroutines for the CDC 6600}

$\operatorname{BUFFER}$ IN $(1,0)(W(I), \dot{W}(120))$ This subroutine reads a record from the input tape into the array, W. IF(UNIT,1) 103, 110, 105, 104 This statement checks the status of the input tape and transfers to 103 if a read is still in progress; to 110 when the read operation is complete; to 105 if an end-of-file is found on the tape; to 105 if a parity error occurs during reading. 
CALL RECALL(1) This statement places SUMTAP3 in an inactive status until the read operation on the input tape is complete. LENGTHF (I) This function computes the length of the record which was just read from the input tape. The record length is usually $80 * 14 / 10=1]$ ? words long.

IF (WARN(IME).NE.O) This statement checks the amount of time remaining to the program. If nonzero, the time limit has almost been reached and SUMTAP3 will terminate gracefully.

Note

The SUMTAP3 program was written in FORTRAN IV and ASCENTF to run on a $\mathrm{CDC} 6600$ computer. If the computer is changed or if the operating system is changed; the program may require revision. 
PROCRAM SUMTAP3 I INPUT, IIUTPUT, TAPE 1, TAPE2= INPUT, TAPE3=OUTPUT,

1 FORMAT $(A 10.215, E 20.13)$

TAPE , TAPE 5, TAPE 98, TAPEY4)

2. FORMAT (4E?C.13)

3 FORMAT (8A10)

4 FORMAT $(1 H 1,25 X, 44 H *$ * ESR TAPE READING AND SUMMINS PROGRAM **\#/I

* 10X,7A10/120X,28HRESULTS IN TAPE4, LABELED \$, A10,2H*\$)

5 FORMAT(315,25X,4A10)

6 FORMAT $/ / / / / 5 X, 4$ HFILE, $13,13 X, 4 A 10 / / 25 X, 14,17 H$ CHANNFLS HETWEEN,

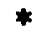
F13.4,4H ANU,F 13.4,5X, 13 HCHANNEL WIDIH,F $12.6 / 1 / 1$

7 FORMAT(2I5, 2E 10.0$)$

8 FORMAT (15X, 1 5HSUMMEO SPECTRUM, 10X, 10HTOTAL AREA, E20.10,5X,

* 15HIINTEGRAL RANGE,E $14.4,3 \mathrm{H}, 1$, E 14.4,1HI//1

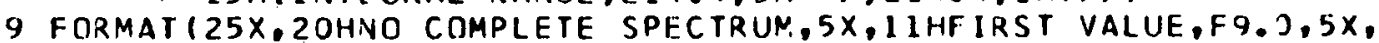

* 10hlast value,fg.01

10 FORMAT ( IHO, IOH****INPUT .FRROR)

11 FORMATI//20X,22HRESULTS STORED AS FILE, I3,9H ON TAPE4/I

12 FORMAT $(10(10 F 12.6 / 1) /)$

13 FORMAT (5HTAPE, A10,5X,4HF ILE, I3)

14 FORMAT $(1,410)$.

15 FORMAT (//15X,26HTOTAL INTEGRATED INTENSITY,E20.10)

16 FORMAT $15 X, 28 H S E C I N D$ ORDER POLYNOMIAL WITH, 13,11 H POINTS AS,

* $\quad$ I8HSMOUTHING FUNCTIIIN, IDX, I5,16H SPECTRA FOUND I, I4,

\$. 12H INCOMPIETEI/I

17 FORMAT(//5X,4HFILE, I3,12H NIIT ON TAPE)

18 FJRMATILHO, 1OX,32HPARITY ERROR ON TAPEI IN RECORD

1 GH FILE , I5 )

19 FJRMATI 1 HO, $16 \mathrm{H}$ YOU FOOL FILE $15,10 \mathrm{I}$ IS EMPTY $/ 1$

$15 X, 5 H *$ * BOH WARNING,-- CHAOS MAY OCCUR -- ASSUMING YOU WISHE

2 D FILE + 1, AND CONTINIJING

20

FORMATI IHO, 2OH CONTENTS OF BUFFER

,

FORMATI $6 X, 10 A 10$ )

DIMENSION COMMENT (7)

OIMENSION FX(250), FY (250),W(120), SZ(2000)

CJMMON/ASPEC/ NSPEC

COMMON/FSPEC/SX(2000) SSY 2000$)$

COMMON/FPAR/I SA, NUM, MM, MN, KP, FOIF, EMIN, EMAX, SW

COMMON/CCPOOL/XMIN, XMAX, YMIN, YMAX, CCXMIN, CCXMAX, CCYMIN,CCYMAX

COMMON/CCFACT/FACTOR

EQUIVALENCE $(S \times(2), F \times(1)),(S \times(321), F Y(1)),(S \times(601), W(1))$

C

DATA KP, NUM, FMIN,FMAX/3,500,300.,9100.1.

INITIALIZATIUN

REWIND 1

REWIND 4

REWIND 5

$X M I N=0$.

$X$ MAX $=10000$.

I $G$ Q I $D=0$

$N E O F=0$

NFILE $=1$

NFILE $2=1$

C

INPUT SECTION

50 READ (2,3) NAMF, (COMMENT $(J), J=1, ?$ )

WRITE $(3,4)$ (C JMMENT $(J), J=1,7)$, NAME

GO READ $(2,5)$ NFILE $1, I K, K K,($ COMMENT $(J), J=1,4)$

IF (NFILEI.LE.O) GOTO 401 


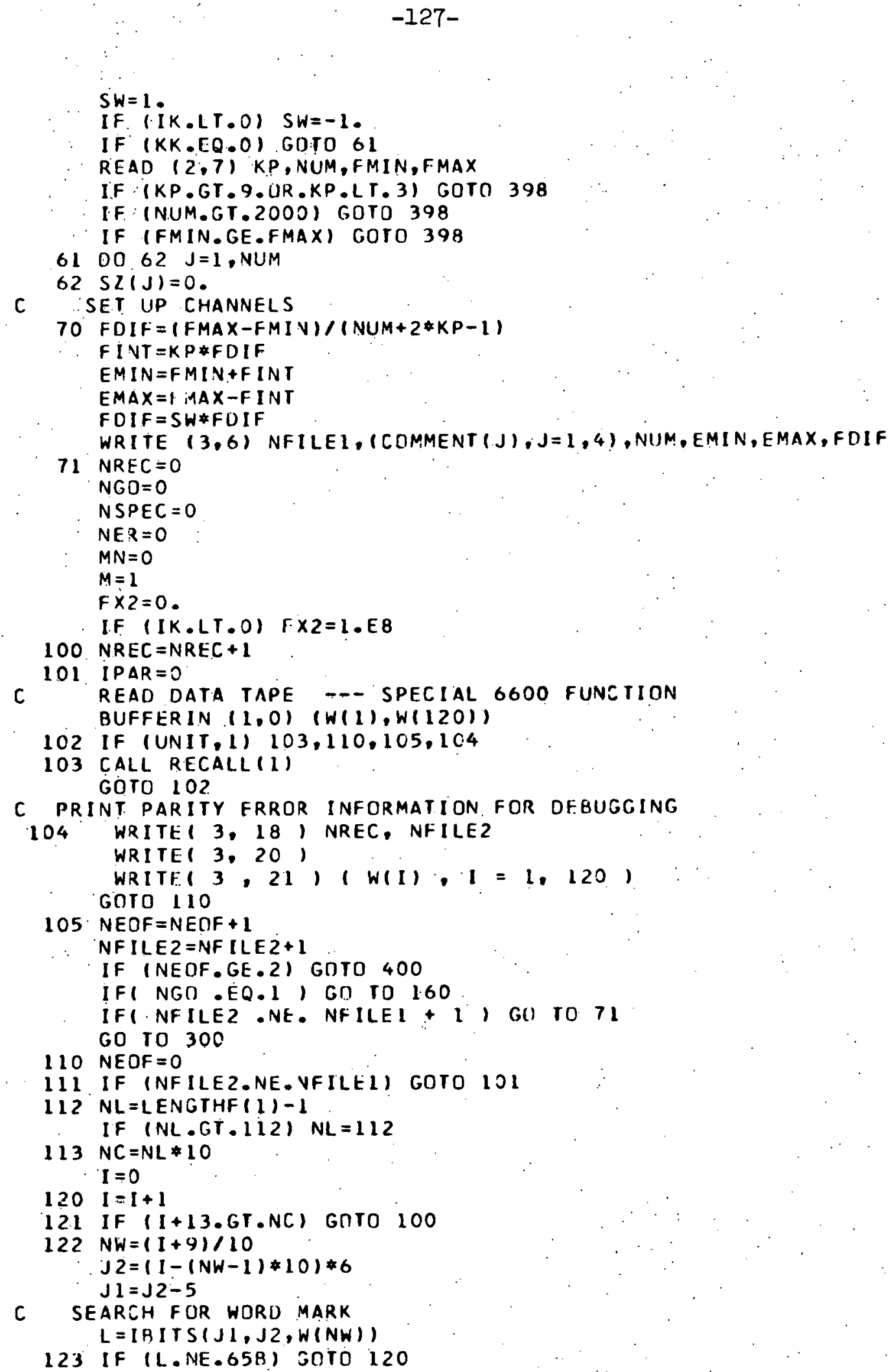




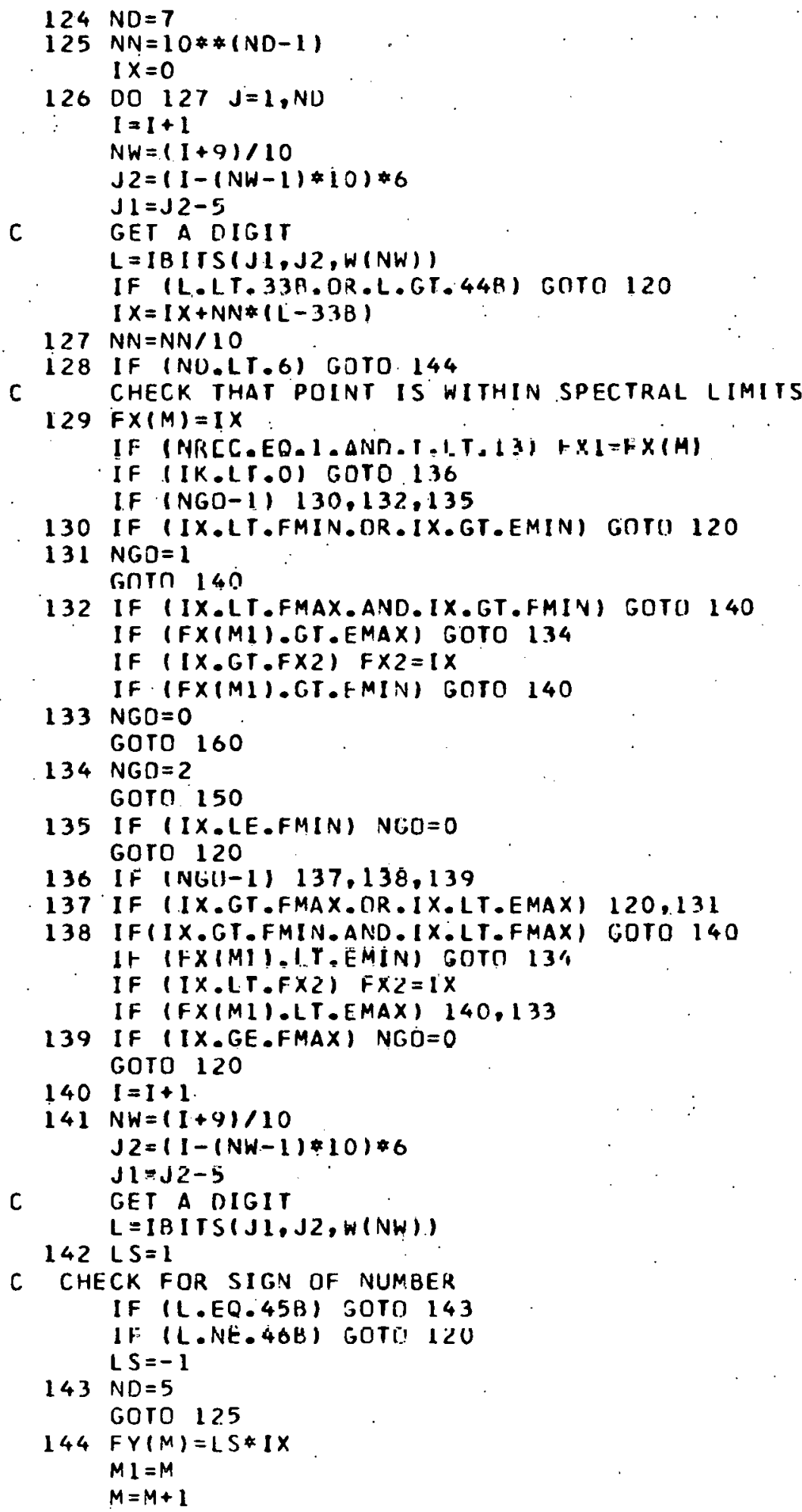




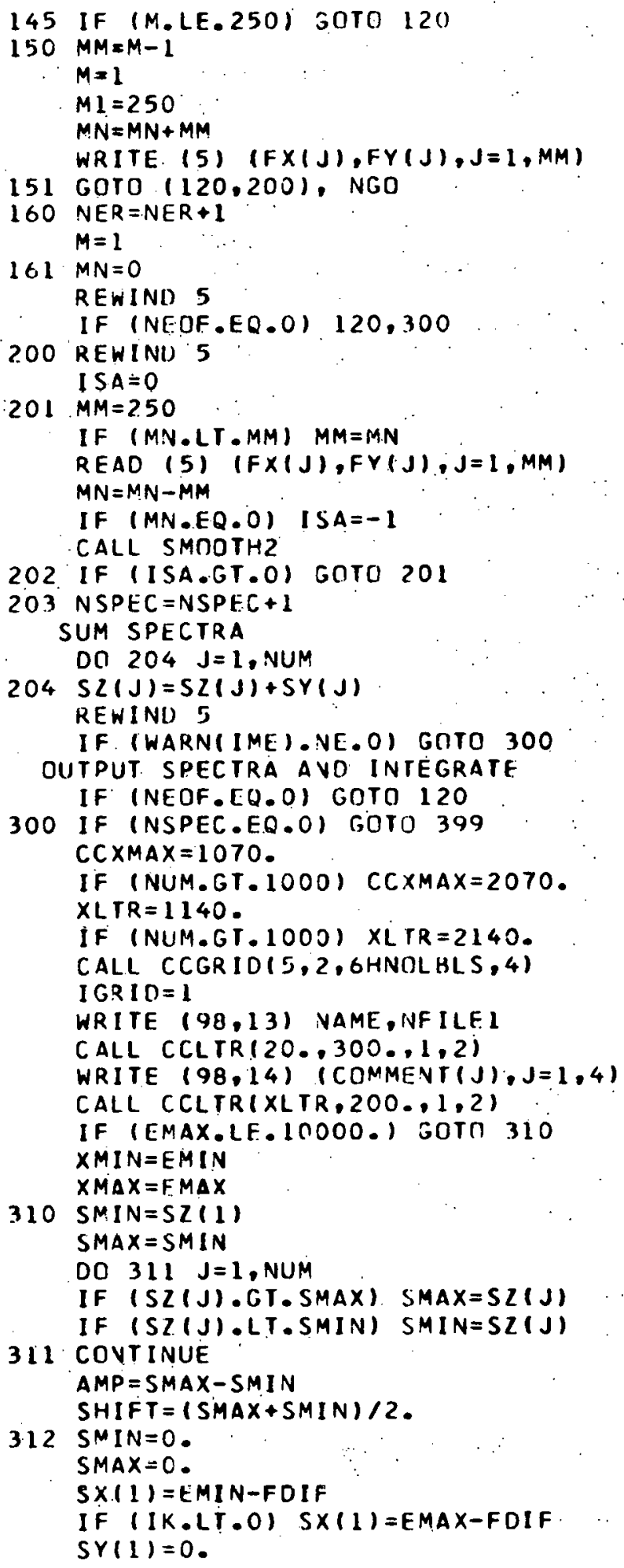




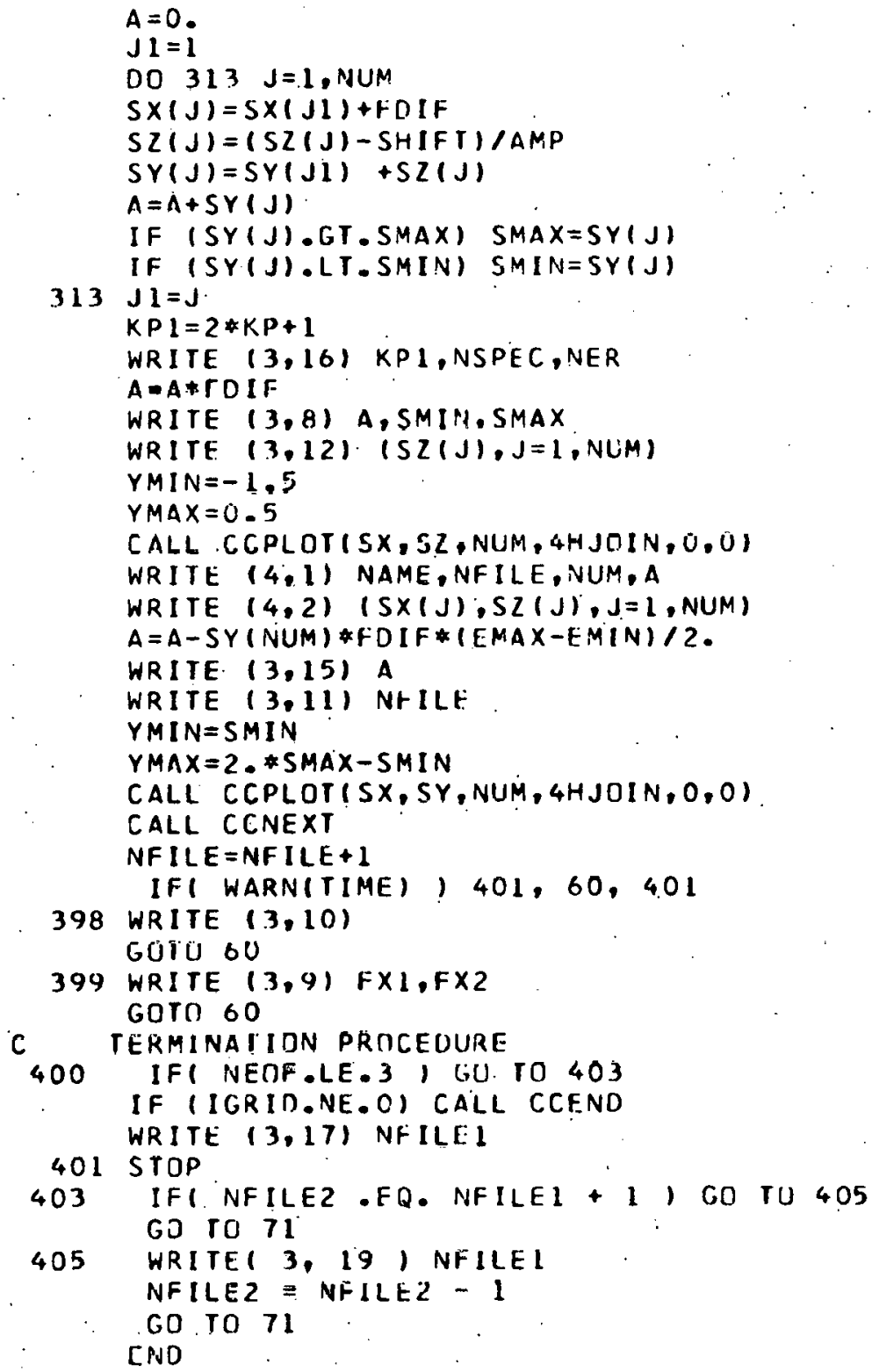


SURROUTINE SMOOTH2

C 2. ORDER SMOOTHING AND INTERPOLATION OF Y-VALUES ACCORDING TO

C 1. ORDER SMOOTHED AND CHECKED X-VALUES COMMON/FSPEC/SX(2000), SY (2000)

COMMON/FPAR/I SA, NUM,MM, MN,KP, FDIF, EMIN,EMAX, SW COMMON/ASPEC/ NSPEC

DATA: $(J=0)$

$1=19-K P$

$I M=M M+20-K P$

IF: (J.GT.O) URTO 2

C

DEFINE SMOOTHING COEFFICIENTS

$1 F A C=2 * * K P+1$

$\triangle O=((K P+1) * K P) / 3$

$A 3=1.1(14 . * K P+4) * K P-3$.

$A I=(19 . * K P+9) * K P-3) * A$.

$A 2=-15 \cdot * A 3$

$A 3=-A 2 / A O$

$E D I F=(E M A X-E M I N) /(M M+M N)$

$G A P=10 . \star E D I F$.

$E D I F=S W * E D$ IF

$F F=E M I N \neq F A C$

IF (SW.LT.O.) FF =EMAX $* F A C$

$D E L=F D I F * F A C$

2. IF (ISA) $10,20,30$

C

TAKE CARE DF GAPS IN DATA

$10 \quad I M=M M+20$

$D O 11 \quad N=1,20$

$N I=N+I M$

$S X(N I)=S X(I M)+N * E D I F$

$11 S X(N)+300)=S X(I \cdot M+300)$

IF (J.NE.O) GUTO 30

20 DO $21 \mathrm{~N}=1,20$

$S X(V)=S X(21)-E 0 I F *(21-N)$

$21 S X(N+300)=S X(321)$

IF (ISA.EQ.O) ISA=1

$I=20$

$30 \quad 11=1+1$.

$T=S \times(I)$

Do $35 \mathrm{~N}=11,1 \mathrm{M}$

IF $(A B S(S X(N)-T) \cdot L T \cdot G A P) \cdot G O T O \cdot 34$

$i=1$

31 I.F $(A B S(S X(N+L)-T) \cdot L T \cdot(L+1) * S A P)$ GOTO 32

$L=L+1$

IF (L.LE.3) GOTO 31

$32 D I F=(S X(N+L)-T) /(L+1)$

DO $33 M=1, L$

$33 S X(N+M-1)=\dot{T}+M * D$ IF

$T=S X(N+L)$

$\mathrm{N}=\mathrm{N}+\mathrm{L}$

Goro 35

$34 T=S \times(N)$

35 COVI INUE

$40 L=1-K P$

$M=I+K P$

$S=0$.

[II) $4 \mathrm{~L} N=L, M$ 


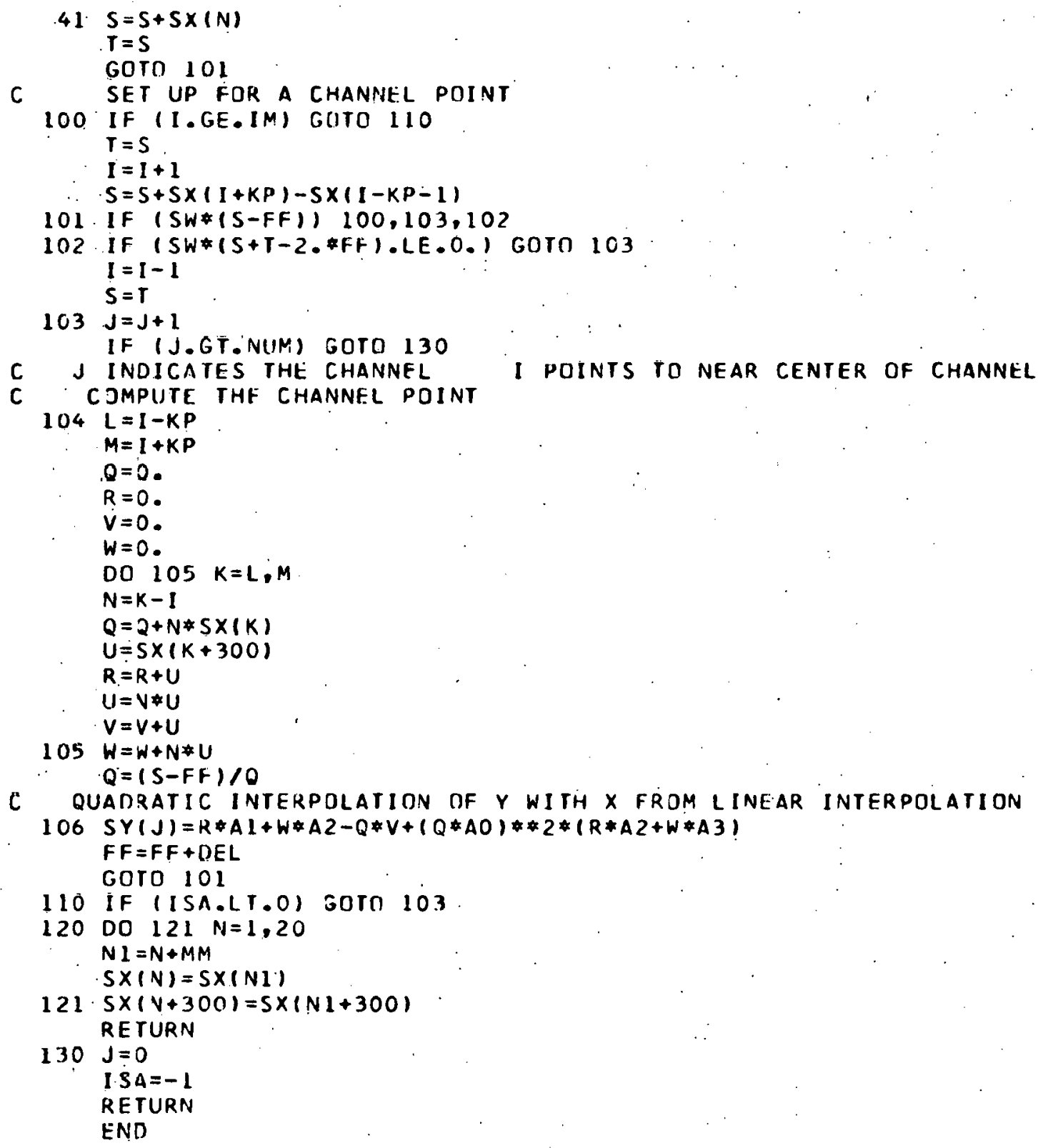




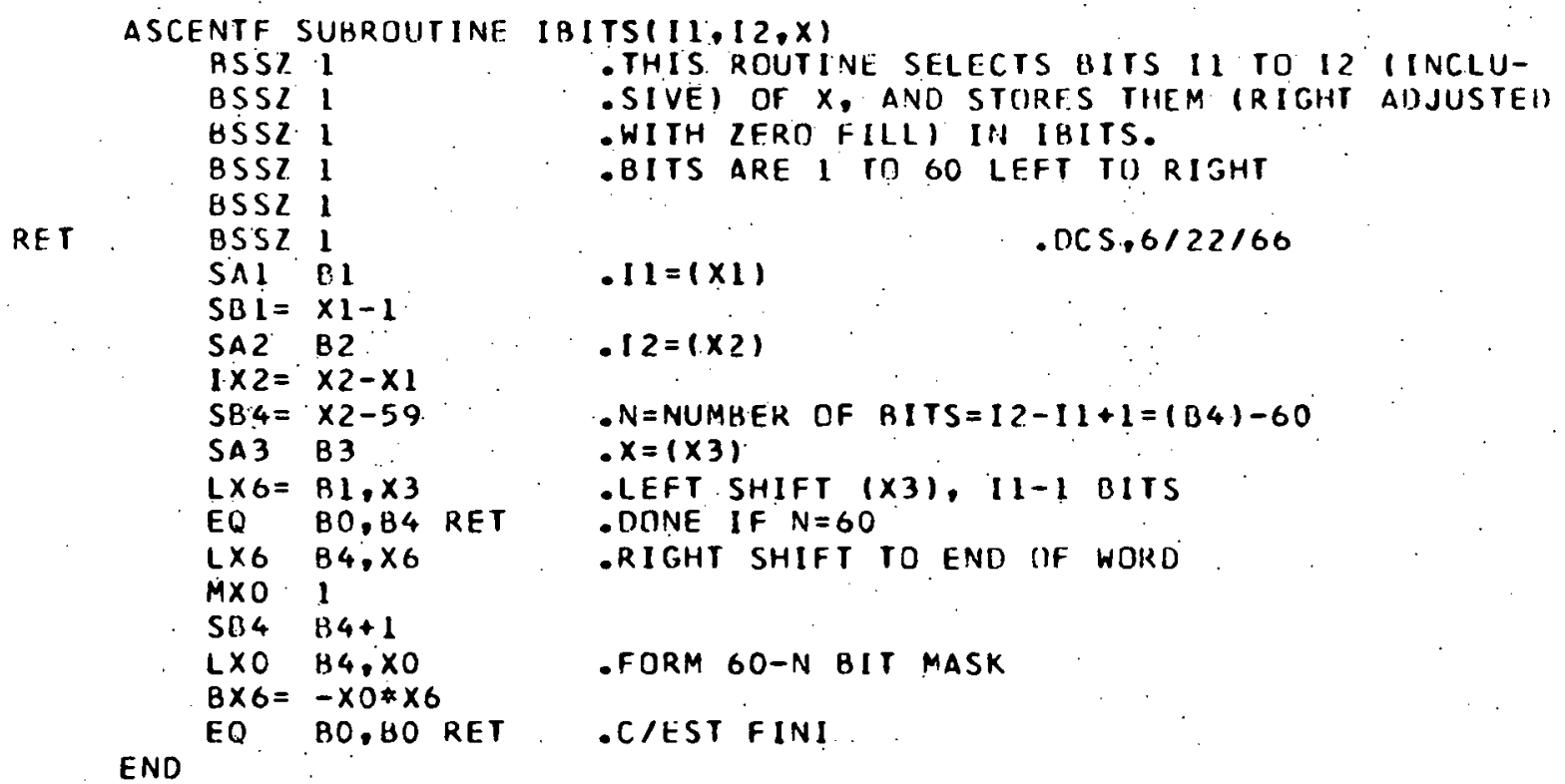




\section{FITESR}

FITESR ${ }^{*}$ performs a least squares fit of Lorentz lineshapes to experimental EPR spectra using the procedure described in Appendix I. It is suitable for spectra with a second order spin Hamiltonian and different widths for the hyperfine components (of I nucleas). It can calculate the relative abundance of up to 5 similar compounds present In a mixture. The spectra are assumed to be stored as derivates of the absorption lines on a magnetic tape produced: by SUMTAP3.

The program operates in the following manner. It starts from a given 0 th order guess of the relevant parameters for the spectrum and iteratively calculates better parameters until the sums of the squares of the deviations between the observed and calculated spectral points remains constant, or until the iteration diverges. The parameters and their corrections in successive steps, including a baseline offset and drift, are printed out. The program prints the final purumeters and plots the observed and calculated spectrum based on these pardmeler's together with enlarged curves of the errors between these spectra.

The program may operate in one of two modes. In the first mode, the spectrum is considered to be composed of up to 12 individual Lorentz lines with different intensities, linewidths, and line centers. In the second mode, the spectrum is considercd to be composcd of hyperf:ne components of one nucleus with the line centers determined by a second order spin Hamiltonian, with the same intensities for all hyperfine components, but with different linewidths. The maximum number of parameters is 38 .

\footnotetext{
*originally written by Dr. Alfred Beuder (Bauder and Myers, 1968).
} 
INPUT DATA

Card 1. (Format 8A10).

NAME The name of the tape with the measured spectra which was given in SUMTAP3. Any 10 alphanumeric symbols (blanks count). This variable is used for file protection and causes the job to abort if it does not agree with the name. found on the tape.

COMMENT Any arbitrary comment to be printed for identification

Card 2. (Format 4I5, 2E10.0, 4AIO)

NFILEl the number of the file to be analyzed (in order of increasing magnitude)

IFIT $:=0$ individual lines according to first assignment of parameters

$>0$ the mber of compounds with second order Hamiltonian

(second mode of operation)

If IFIT is 0 ; the first mode of operation is selected; otherwise, IFIT is the number of compounds to be fit with a second order Hamiltonian

NLINE .. the number of hyperfine components. If IFIT $=0$, then NLINE is the number of individual lines to be fit.

KFIELD $=0$ use abritrary field calibration without nonlinearity correction.

$>0$ use arbitrary calibration with nonlinearity correction

$<0$ use the HCALIB field calibration subroutine.

This is a historically provided option. Usually KFIELD is negative and the HCALIB subroutine is used to calibrate the magnetic field. 
HSTART Lower limit of the field sweep in gauss

HSTOP Upper limit of the field sweep in gauss.

If KFIELD is negative, the HCALIB routine is used, and HSTART and HSTOP are not used. They are only used if KFIELD is zero or positive.

COMMENT . Any comment to be printed and plotted to jdentify the job.

Card 3. - and following cards (Format BFIn.n)

$X(J)$ the 0 th order estimates of the parameters

if IFIT=0; first, all the line centers, second all the intensities and last all the linewidths of the NLINE individual absorption lines in the same order.

if IFIT > 0; for each compound separately, in the following order; first, the $H_{0}$ field, second, the hyperfine coupling constant, third, the integrated intensity, and last all the linewidths of the hyperfine components ordered according to ascending azimuthal quantum number.

In both cases, the last two parameters are the baseline offset and baseline drift.

Cards are repeated from Card 2 to analyze additional snertra. A Vlank card at the end terminates the program execution.

SUBROUTINES

HCALIB $(K, N, F)$ calibrates the $N$ points in the array $F$ according to the calibration specified by $K$ (see listing)

MATINV ( $A, N, B, M$, DETERM) solves the matrix equation $A X=B$ for $X . A$ is an $N \times \mathbb{N}$ matrix. $M$ is the number of column vectors in $B$ and should 
be one. DETERM contains the determinant of $A$ after the equations have been solved. The solution $X$ is returned in $B$, and the inverse of $A$ is returned in $A$.

NOTE

If the data for the spectrum is to be input from cards rather than from magnetic tape, the PROGRAM card must be altered so that the TAPE4 specification is changed to TAPE4=INPUT. The data cards for the spectra must be placed after card 2. The format of the data can be obtained from an examination of the program listings. 
PROGRAM FITESR I INPUT, DUTPUT, TAPE $2=$ I NPUT, TAPE $3=$ DUTPUT, IAPE 4,

1 TAPE98.PI.DT, TAPE99=PLOT ।

DATA (LOUP $=0),(P I=.636619772 .367581)$

DIMENSION G(38),X(38),FX(1000),FY(1000),FZ(1000), H(38,38)

DIMENSION COMMENT (7), AR(5),GR(38), SP(33), IC (33)

DIMENSIUN FC $(33), C(33), 0)(33), 1) 0(33), U S(33)$

DIMENSION GI $(38)$

DIMENSIION FW(1000)

EQUIVALENCE $\left(H, F H^{\prime}\right)$

COMMON /CCPOOL / XMIN, XMAX, YMIN, YMAX, CCXMIN, CCXMAX, CCYMIN,CCYMAX COMMON/CCFACT/FACTOR

1 FORMAT (8A1O).

2 FORMATIIHI, $10 X, 7 A 10 / / 21 X, 37 H * *$ LEAST SOUARES FIT TF ESR-SPECTRA. * 3H*\$//20X,28HSPECTRA ON TAPE4, LABELED * *A10,21\%*//11

3 FORMAT(4I5,2E10,0,4A10)

4 FORMAT I///5H FILE, 13,10X,17HESR-SPECTRUM WITH, 13.

* 28H [NOIVIDUAL LDRENTTION I INESIII]

5 FórMATI///5H FILE, $13,10 X, 15 H E S R-S P F C T K U M$ OF, 12,13 H SUHSTANCES,

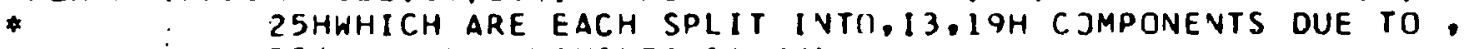

* 24HSECOND (IRDER HAMILTONI AN//I

6 FORMAT $18 \times, 3$ OHNUMBER OF FITTING PARAMETERS 1,12,

* 17HI OR SUBSTANCES 1,12,2H1.

* 26HEXCEEDS AVAILABLE IAPACIIY//I

7 FORMAT $(A 10,215, E 20.13)$

8 FORMAT $(18 \times, 4 \mathrm{HFILE,} 13,13 H \mathrm{~N}$ NT ON TAPF, $A 10 / /)$

9 FORMAT $(10 X, 21 H I N C O R R$ ECT.FILE LABEL, A10)

10 FORMAT (4E20.13)

11 FORMATI $20 X, I 4,2$ OH DATA POINTS ON FILE,I $3,5 X, 12$.

*

12 FORMAT (8E 10.0) I9H FITIING PARAMETERS///)

13 FORMAT (5HTAPE , A10,5X,4HF ILF, 13)

14 FORMAT (1OX,33HFINAL VALUES OF LEAST SQUARES FIT,

* 36H ISTANDARo deVIaTION IN PARENTHESISI//10X.

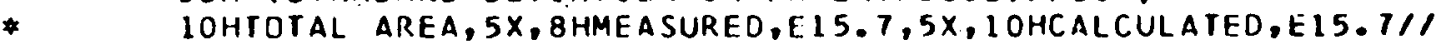

$\$ \quad 10 X, 15 H B A S E L I N E(A+B X), 12 X, 1 H A, E 15,7,2 H\left(, E(5,7,1 H), 9 X_{0}\right.$

5 LHE,F15.7,2.H (,E15.7,1H//)

15 FORMAT $110 X, 9 H C$ JMPONEN $1,10 X, 11$ HLINT. CENTIR, $18 X, 9 H I$ NTENSITY, $21 X_{0}$ * . 9HHALFWIDTH/)

16 FORMAT $(13 X, 12, F 18,4,2 H(, F 8,4,1 H), E 17.5,2 H(, F 12.5,1 H)$,

* F(5.4,2H (,F8.4,1H))

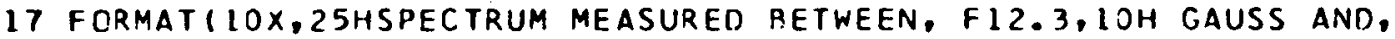

* F12.3.6H GAUSS///25H ABSOLUTE VALUES IN GALSS//)

I\& FORMAT(3X,3HNO, ,7X, 14HREL. INTENSITY,8X,17HHO-FIELD (CENTER).

* 9X, 1 HASPLITTING CIINSTANT, SX,5HCOMP.,10X, IOHLINE WIDTH.

\$ $9 \times, 11$ HLINE (ENTER/)

19 FORMAT $(3 X, 12, F 13,4,2 H(, F 6,4,1 H), 2(F 15,3,2 H(, F 8,3,1 H), 18$,

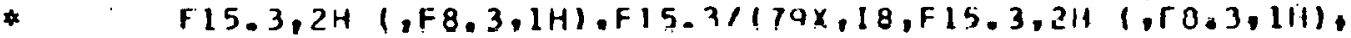

s F(5.3)1

20 FORMATI10X,34H* FATAL ERROR THRMINATES JOF *\$)

21 FORMATI///34II RELATIVE VALUES REFERKING TO PLOT//)

22 FORMAT ( $/ 20 X, 14 H I T E R A T I O N$ STEP, 12,5X,2HF $=, E 20,12,5 X, 2 \mathrm{HX}=1(10613.4))$

23 FORMAT ( $/ 20 X, 14$ HITERATION STEP, I $2,9 H$ DIVERGED)

24 FORMAT $(/ / /)$

25 FORMAT ( $20 X, 11$ HCDRRECTIONS, $5 X, 2 H G=1(10 E 13.4)$

26 FORMAT $(20 X, 2 H * *, 4 \Lambda 10,2 H * * / 1)$

2.1 FORMAT (4A10) 


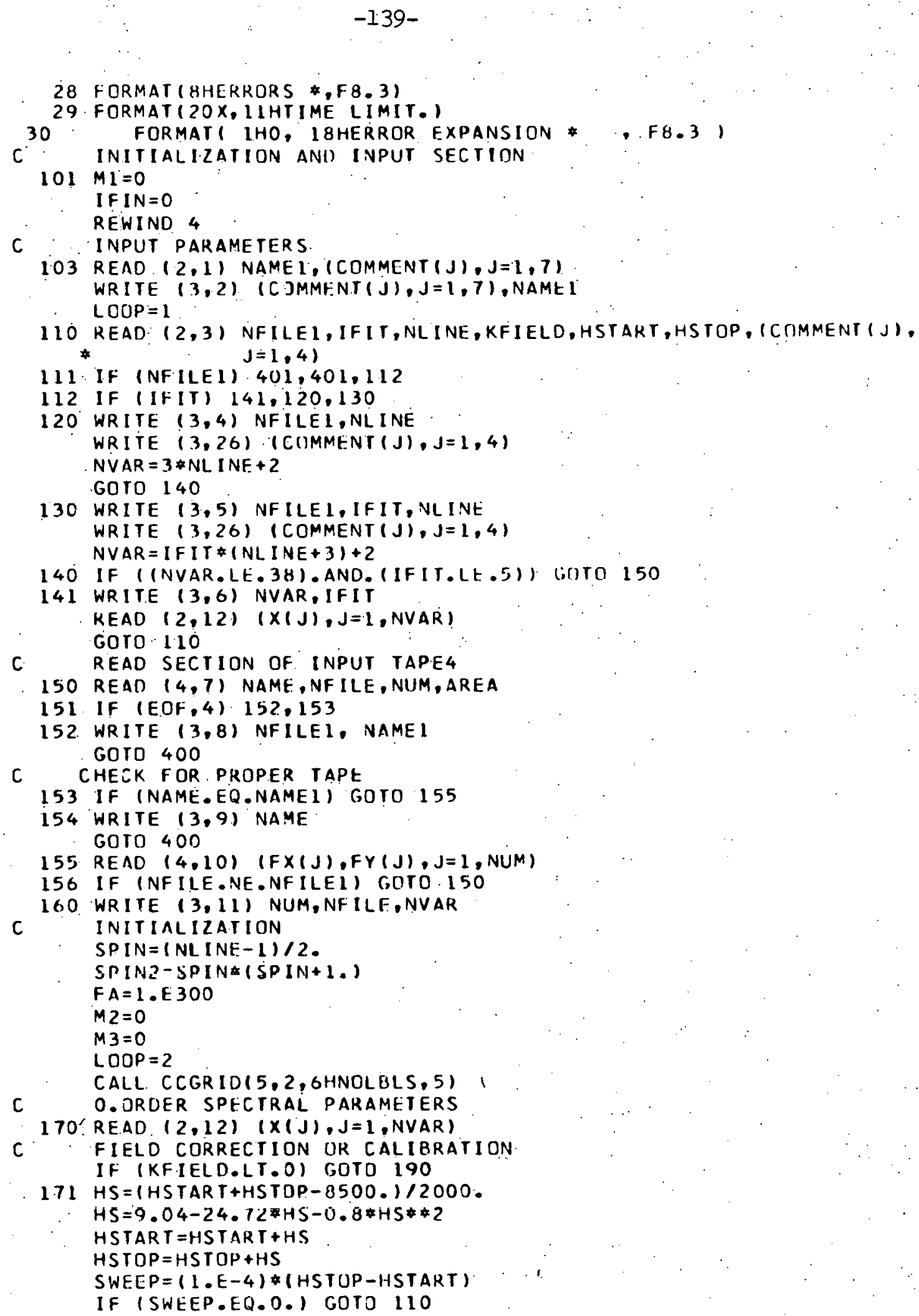




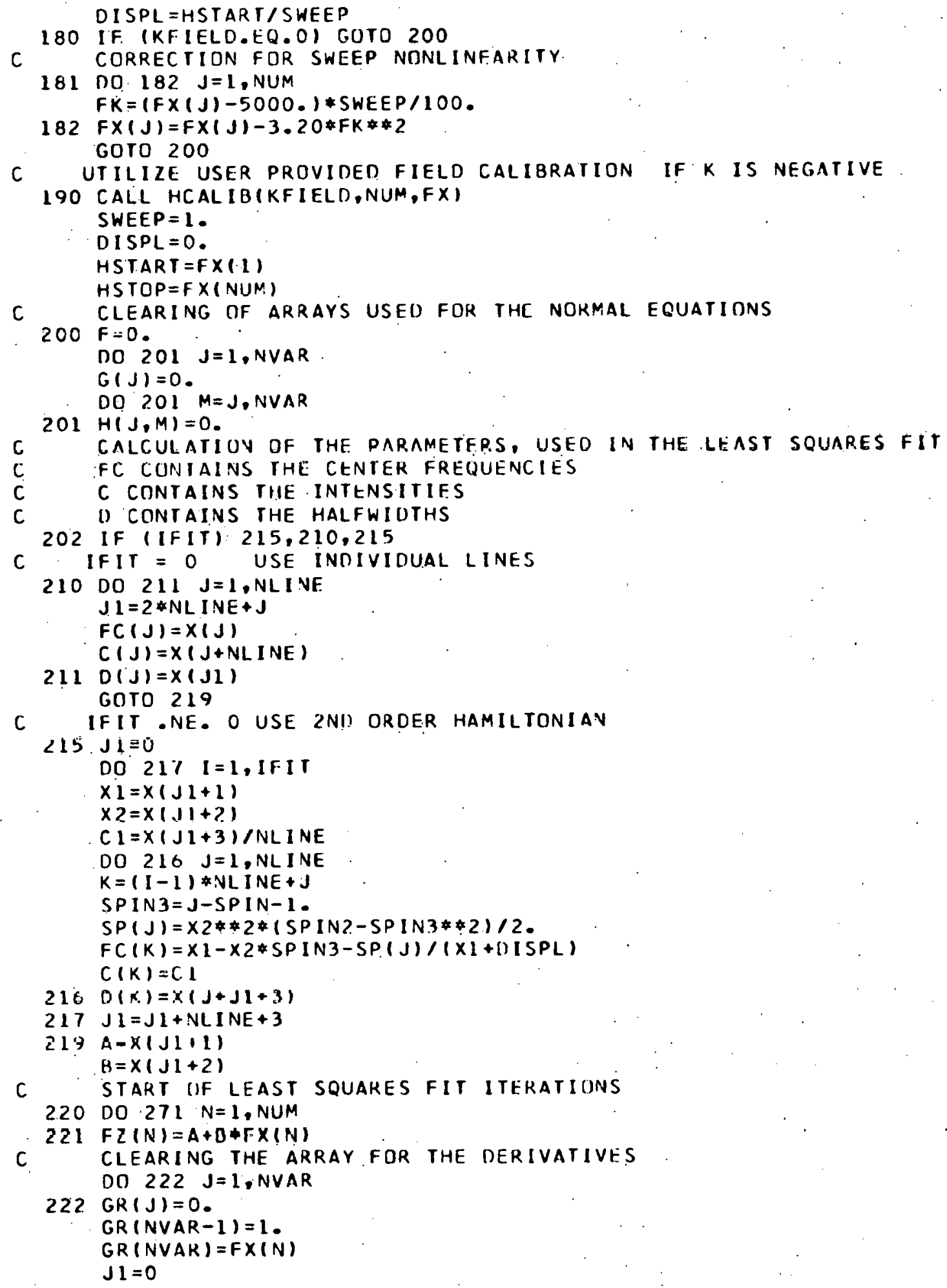




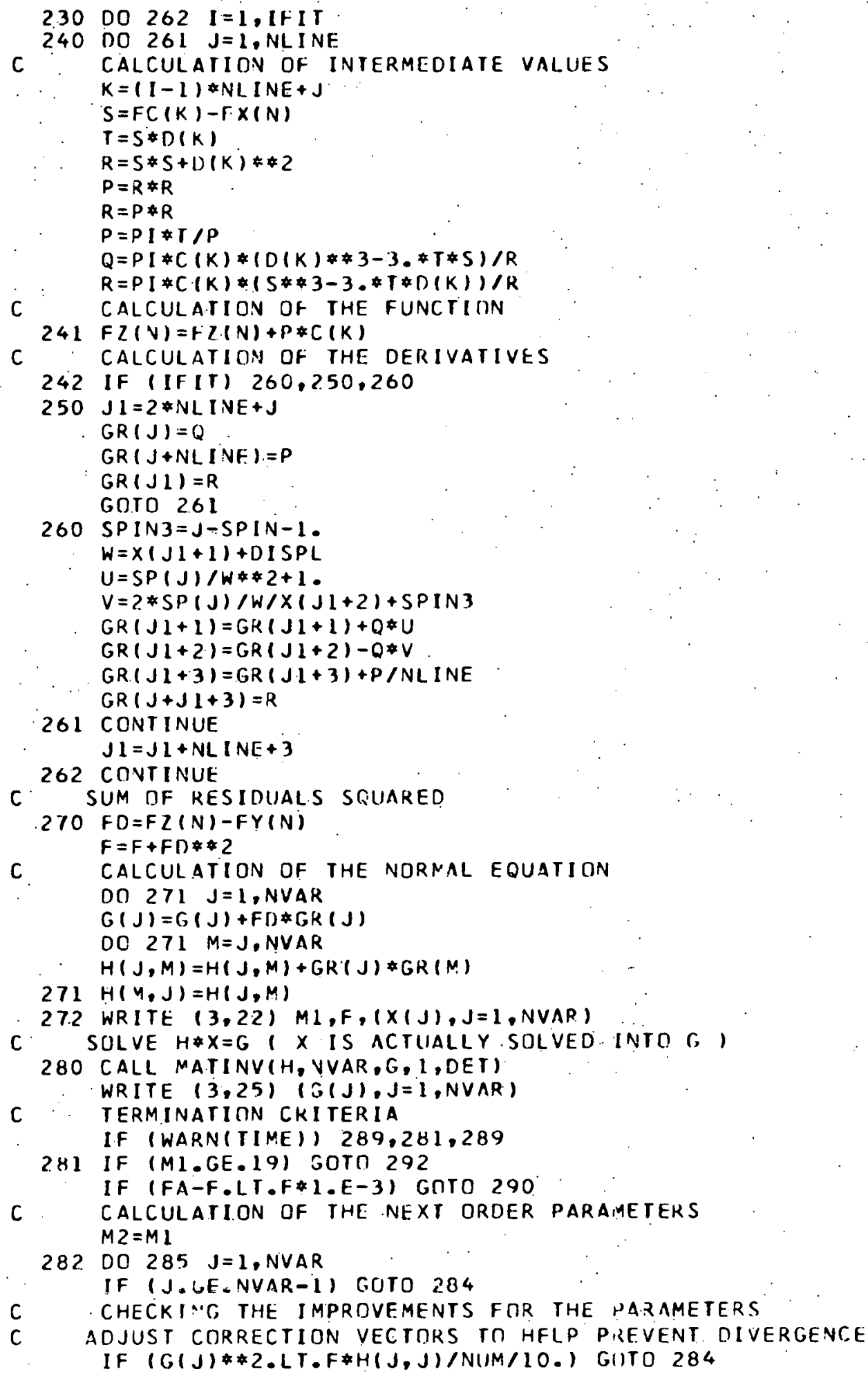




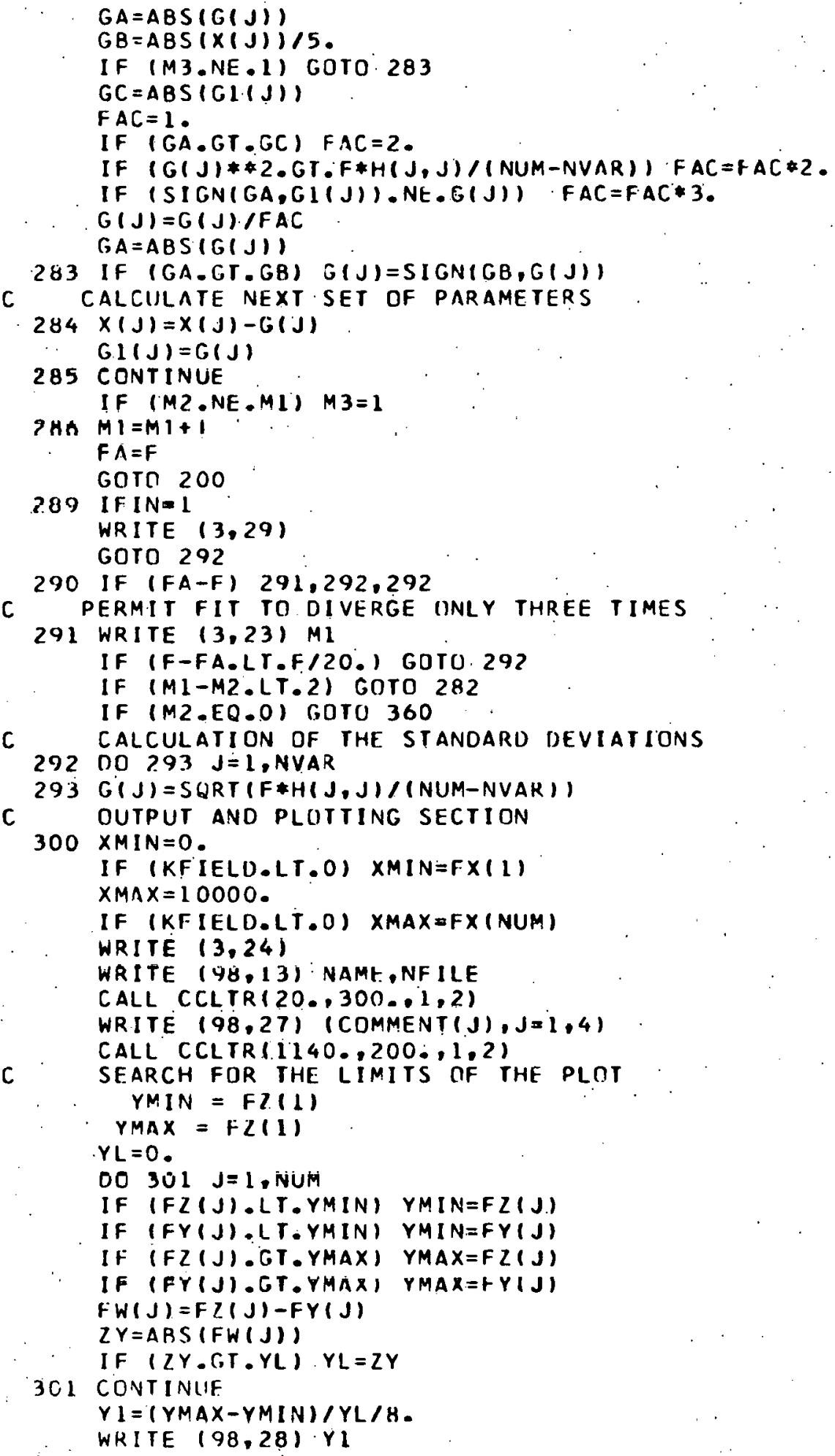


CALL CCLTR $(70,30,0,2)$

$Y M I N=(5 . * Y M I N-Y M A X) / 4$

CALL CCPLOTIFX,FZ,NUM, 4HJOIN,0,0)

CALL. CCPLOTIFX,FY,NUM, 6HNOJOIN,23,3)

$Y M_{1} I N=-Y L$

$Y M A X=9 . Y L$

EALL. CCPLOTIFX,FW,NUM, 4HJOIN, O,O)

CALL : CCNEXT

C

CHECKING OF THE INTEGRATED INTENSITIES.

310 ARE $=0$.

DO 312 I = I, IF I T

$A R(I)=0$.

DO $311 \mathrm{~J}=1$, NLINE

$K=(I-1) * N L I N E+J$

$311 A R(I)=A R(I)+C(K)$

$312 A R E=A K E+\cap R(I)$

$313 Y=F X(N U M)-F X(1)$

$A R A=A R E+A * Y * 2 / 2 \bullet+B * Y * \$ 3 / 6$.

320 WRITE $(3,14)$ AREA,ARA,A,G(NVAR-1), B,G(NVAR)

C OUTPUT OF THE RESULTS

321. I.F. (IFIT) $350,340,350$

340 WRITE $(3,21)$

WRITE $(3,15)$

DO $341 \mathrm{~J}=1$, NL INE

$C . T=G(J+N L I N E)$

$D T=G(J+2 * N L I N E)$

341 WRITE $(3,16) \mathrm{J}, F C(J), G(J), C(J), C T, 1)(J), D T$ GOTO 360

350 WRITE $(3,17)$ HSTART,HSTOP

WRITE $(3,18)$

$\mathrm{J} 1=0$

DO $352 \quad 1=1$, IF I T

DO $351 \mathrm{~J}=1, \mathrm{NL}$ INE

$K=(\lfloor-1) * N L I N E+\rfloor$

$I C(K)=J$

$D D(K)=D(K) * S W E E P$

$S P(K)=G(J+J 1+3)$

$D S(K)=G(J+J 1+3)$ *SWEEP

$351 C(K)=F C(K)$ \&WEEP+HSTART

$K=(I-1) * N L I N E+1$

$K I=K+N L I N E-1$

$F H=H S T A R T+X(J 1+1)$ \&WEEP

$F G=X(J 1+2) *$ SWEEP

$F S=G(J 1+1)$ *SWEEP

$F T=G(J 1+2) *$ SWEE $P$

$A R(I)=A R(I) / A R E$

$G R(I)=G(J 1+3) / A R E$

I.F (KFIELD.LT.0) GOTO 352

WRITE $(3,19)$ I, ARII),GR(I),FH,FS,FG,FT, IIC(J),OD(J),DS $(J), C(J)$,

* $J=K, K l)$

$352 \mathrm{~J} . \mathrm{I}=\mathrm{J} I+\mathrm{NLINF}+3$

IF (KFIFI. D.LT.O) G(ITO 353

WR ITE $(3,21)$

WR ITE $(3,18)$

353. $\mathrm{Jl}=0$

DO $354 \quad I=1$, IF I T 


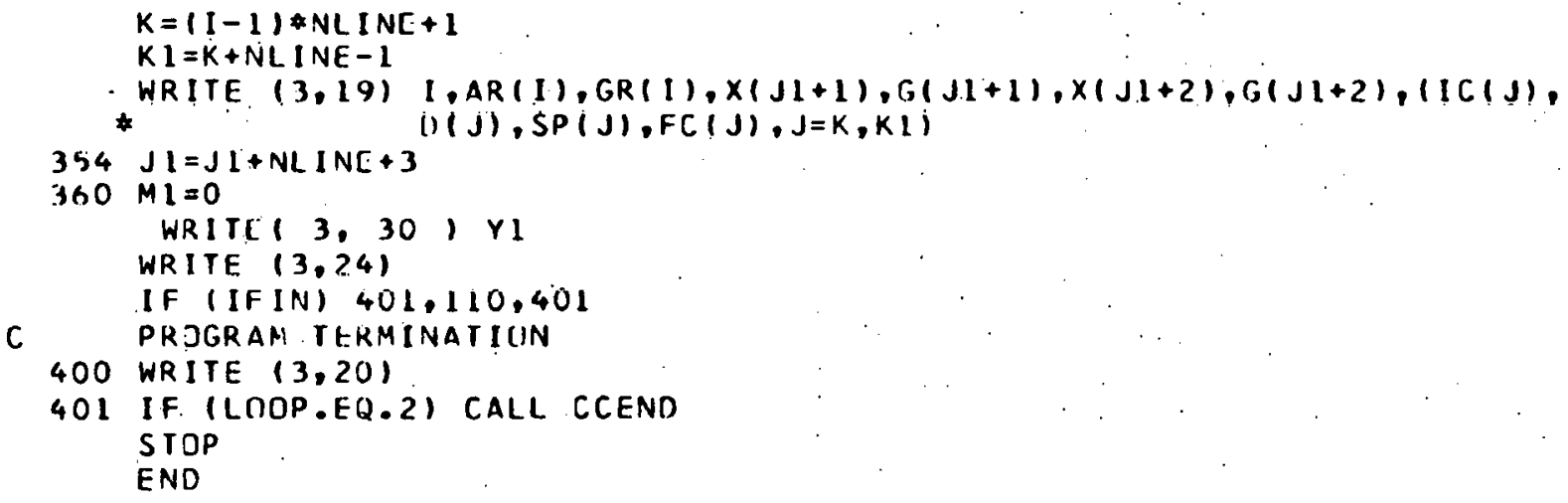


SUBROUTINE HCALIB ( K.N.F) .

DIMENSION F( LOOO)

C SAMPLE LALIBRATION ROUTINE

C CALIBRATE THE VALUES IN F N POINIS

C O OR CONVERT FROM ONE UNIT TO ANITHER UNIT

$C$ FOR EXAMPLE IF F CONTAINS NMR FREQUENCIES

C. DO $10 \mathrm{~J}=1 . \mathrm{N}$.

C $10 F(J)=F(J) / 425775$

C FOR FITLDIAL CALIBRATION WITH COEFFICIENTS FROM PROGRAM GAUSS

C DO $10 \quad J=1, N$

C $10 \quad F(J)=A+B * F(J)+C * F(J) * 2$

C IF JATA REQUIRE SEVERAL DIFFERENT CALIHRATICNS

C K MAY BE USEO TO SELECT CALIRRATION

C IK $=-K$

C GO TO $(1,2,3, \ldots$ ETC....) IK

10 $0010 \mathrm{~J}=1, \mathrm{~N}$

$10: F(J)=2834.7580+11.0366706 E-01-2.2958270 E-07 * F(J), * F(J)$ RETUKN END 
FORTRAN IV SUBROUTINE MATINV $(A, N, B, M, D E T E R M)$

F4020003

F4020004

$c$
$c$

MATRIX INVERSION WITH ACCOMPANYING SOLUTION OF LINEIR EQUATIONS

ANF 40201

DIMENSION IPIVJT $(38), A(38,38), B(38,1)$, INDEX $(38,2), P$ IVOT(36)

EQUIVALENCE (IROW,JROW), (ICJLUM, JCOLUM). (AMAX, T. SWAP)

F 4020002

$\mathrm{C}$
$\mathrm{C}$
$\mathrm{C}$

INITIALIZATION

10 DETERM $=1.0$

15 DO $20 \mathrm{~J}=1, \mathrm{~N}$

20 I PIVOT $(J)=0$

C

$3000550 \quad I=1, N$

F4020007

$F 4020008$

14020009

F4020010

$F 4020011$

54020012

F 4020013

F4020014

F4020015

SEARCH FOR PIVIT ELEMENT

SEARCH FOR PIVJI LLEMENT

$F 4020016$

F4020017

F4020015

$F 4020016$

$F 4020017$

40 AMAX $=0.0$

45 DO $105 \quad J=1, N$

50 IF (IPIVOTIJ)-1) OU, IUS, bU

60 DO $100 \mathrm{~K}=1, \mathrm{~N}$

70 IF (IPIVOI(K)-1) $80,100,740$

BO If (ABS(AMAX)-ARS(A) J,K)) 85, 100, 100

$85\lfloor R J W=J$

90 ICOLUM $=K$

95 AMAX $=A(J, K)$

100 CONT INUE

105 CONTINUE

IF (AMAX) $110,800,110$

110 IPIVOTIICOLUM ) = IPIVOT (ICOLUM +1

$\mathrm{c}$

INTERCHANGE ROWS TO PUT PIVOT ELEMENT ON DIAGONAL

$F 4020018$

$F 4020019$

$F 4020020$

F4020021

F4020022

$F 4020024$

14020025

$F 4020026$

$F 4020027$

F4020028

F402REV.

$F 4020029$

$F 4020030$

F 4020031

F 4020032

$F 4020033$

130 IF (IROW-ICOLUM) 140,260, 140

140 DETERM =-DE TERM

150 DO $200 \quad L=1, N$

160 SWAP $=A(I R O W, L)$

170 A (IROW, L) =A (ICOLUM,, )

200 AIICOLUH,LI = SHAP

205 I $F(M), 260,260,210$

$21 C$ DO $250 \quad L=1, M$

220 SWAP $=P\left(I R O W_{0} L\right)$

$230 B(I R O W, L)=B(I C O L U M, L)$

$250 B(I C O L U M, L)=$ SWAP

260. INDEX $(1,1)=\{$ ROW

270 INDEX $(1,2)=1$ COLUM

310 PIVOT (I)=AIICCLLU, ICOLUR)

c

320 DETERM =DE TERM $\$$ PIVOT (I)

C DIVIDE PIVOT KOW BY PIVOT ELEMENT

c

330 A $(I C . O L U M, I C O L U M)=1.0$

340 DO $350 \quad L=1, N$

$350 \hat{A}(I C O L U M ; L)=A I$ ILULUM, LI/PIVOTII)

$F 4020034$

$F 4020035$

$F 4020036$

F 4020037

$F 4020038$

F4020039

$F 4020040$

$F 4020041$

F 4020042

F4020043

F4020044

$F 4020045$

F4020046

F4020047

54020048

F 4020049

F4020050

F4020051

F4.220ก5?

$F 4020053$ 


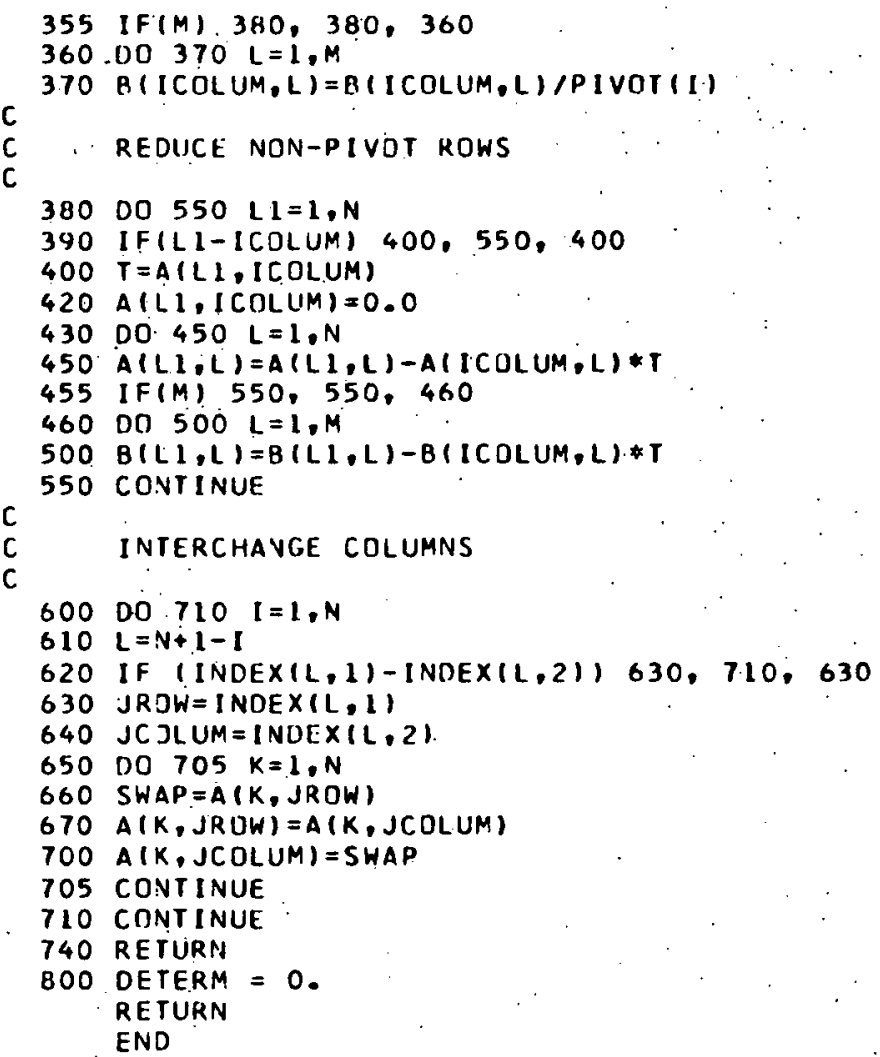




\section{GAUSS}

When the digital data acquisition system is used with the Varian spectrometer system, a MMR tracking system is not normally employed. In this case the recorder $x$-axis drive voltage from the Fieldial is applied to a Vidar voltage-to-frequency converter and this frequency is measured as the magnetic field coordinate. In order for the least squares fitting programs to work in units of gauss, a calibration of the vidar frequencies in terms of magnetic field must be performed. The GAUSS program relates the Vidar frequencies to the magnetic field.

A series of measurements of the magnetic field (using proton MMR) is made over the entire range of the sweep. This produces a set of paired measurements of the magnetic field (NMR frequency) and of the Vidar frequency. A quadratic equation

$$
Y=A+B * X+C * X^{* * 2}
$$

if fit to these points to produce the coefficients $A, R, C$. Then, given a Vidar frequency $(X)$, the magnetic field $(Y)$ may be computed. These coefficients are used to write the subroutine HCALIB which is used by FITESR. The input to the program is the measurements $X(I)$, the Vidar frequency; and $Z(I)$, the proton frequency corresponding to $Y(I)$ the magnetic field in gauss. The proton MMR frequency is converted to gauss by the program. The output consists of the coefficients A, B, and C and a comparison of the experimental field with the theoretical field for a given vidar value. 


\section{INPUT DATA}

Card 1 (Format I5, 7AlO)

N . number of pairs of Points used in the calibration (minimum of three)

TITLE . any identification information

Card:2 (Format 8E10.0)

$X(I) \quad$ Vidar reading in terms of tens of cycles (decacycles)

$Z(1) \quad$ Proton NMR frequency in kilocycles

$X(2) \quad$ etc. until $X(N), Z(N)$

$z(2)$

There are as many of Card 2 as needed to contain $N$ points, 4 points to a card.

If several calibrations are to be performed at once, continued with data from Card 1, etc. A blank card at the end of the data will terminate processing.

The GAUSS program requires the subroutine MATINV which is described in the discussion of FITESR. 


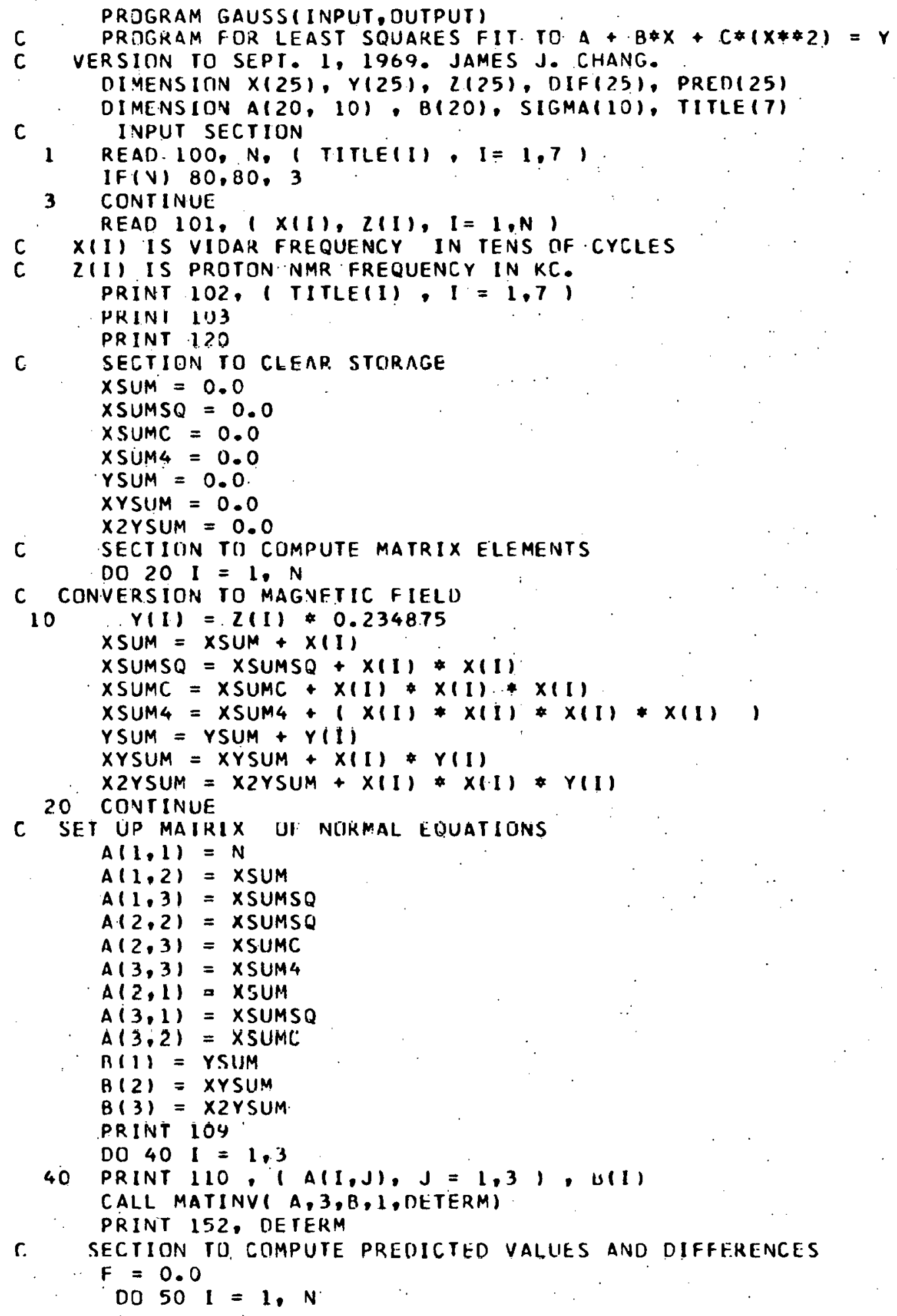




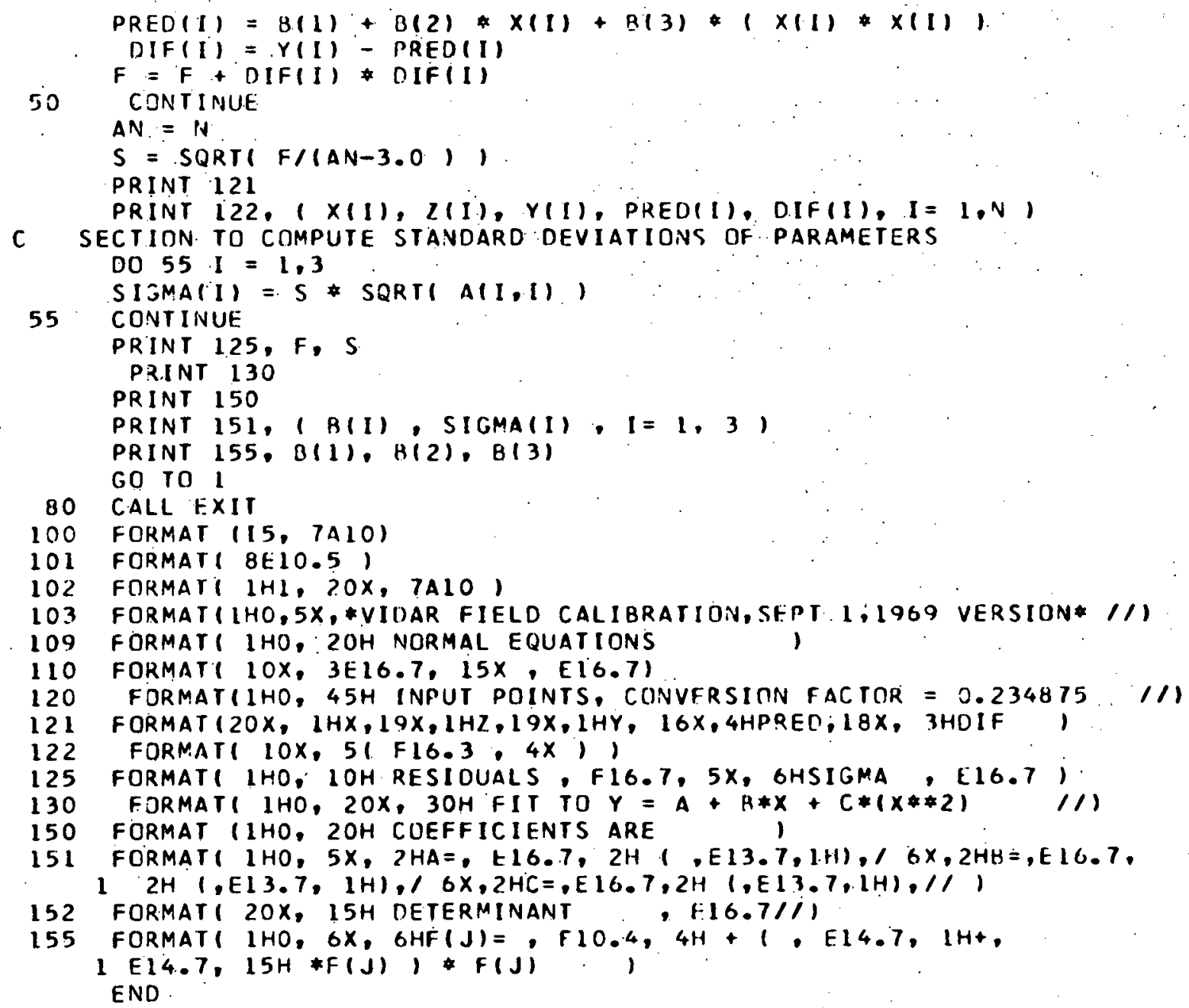




\section{B. ' Simulation Programs}

\section{IMITATE}

The IMITATE program is used to simulate isotropic EPR spectra for both transition metals and organic radicals. The line positions are determined by a $g$ value and the coupling constants according to Eg. A-ll. The lineshapes are produced by Eq. A-l2 which is expanded to account for more than one species. The program is at present capable of simulating a spectrum containing as many as four separate spectra each of which is characterized by $g$ values and A values. The program is well suited for simulating spectra of a system containing several isotopic species.

In operation, the program first computes the resonance fielcis for each of the lines in the spectrum. Because of the storage limitations the program is limited to a maximum of 1600 lines. This may be changed by changing the DIMENSION statements. The intensity ratios of the: lines are computed by SUBROUTINE RATIO. Again because of dimension Iimitation a given coupling constant should not produce more than 25 Iines.

\section{INPUT DATA}

Card 1 (Format 2I5, 30X, 4AIO)

NSPECY The number of species to he simulated. Maximum value of 4 . If NSPECY $=0$, the Job is terminated.

NUM The number of points lu be computed in the spectrum COMMENT $(\mathrm{J})$ Any comment information

Card 2 (Format 10I.5)

NCOUP $(J)$ The number of coupling ronstants for each species. Fur one species there is NCOUP (1); for two, there are NCOUP ( 1 ) and $\operatorname{NCOUP}(2) . \operatorname{NCOUP}(1)=1$ for one coupling constant; =2 for two constants, etc. 
Card 3. (Format 5(I5, F10.5))

$\mathrm{NEQ}(J !, J)$. The number of equivalent nuclei corresponding to a particular coupling constant. (which is given later), for the Jth species. $A l l$ of the $N E Q(J I, J)$ for the same species are on the same card. $S(J l, J)$ : The nuclear spin for the NEQ nuclei. For two protons $N E Q=2 ; S=0.5$.

There are as any of card 3 as there are species. Note that if there are more than 5 sets of equivalent nuclei for a given species, the date. must be on a second card 3 (maximum of 10 . sets possible).

Card 4. (Format 8F10.5)

HSTART : The starting field for the simulation

HSTOP The ending field for the simulation. The spectra are computed from HSTART to HSTOP

ALPHA : Baseline off set

BETA . Baseline drift. Normally ALPHA and BETA are zero.

Card 5. (Format 8F10.5)

FINT(J). The intensity of the Jth species. This is an arbitrary nunber but the relative values of FINT shoild correspond to the relative intensities of the different species.

HZERO $(J)$. The resonance center for the Jth species. This should be computed from the $g$ value and the microwave frequency.

$A(\dot{J} l, J)$ The coupling constants corresponding to the $N E Q(J I, J)$ of card 3 . for the Jth species. More than 6 constants are continued cn the next card.

Card 6. (f'ormat 8F10.5)

FLAG =0 read the widths of all of the lines f0 all the lines have the same width 
Card 7. (Format 8F10.5)

$\mathrm{W}(\mathrm{K})$ The linewidth. If $F L A G=0$, the widths for all the lines must be provided on as many of card 7 as required. For the purpose of identifying a given line, each line is identified by a $K$ value in addition to its identification by the $m_{I}$ values. The value of $K$ is determined by the $m_{I}$ values and by the order in which the spins were presented on card $3 . \mathrm{K}=1$ occurs when all the $m_{I}$ have their lowest values. When the first $m_{I}$ (corresponing to the first $S$ on card 3) has the second from lowest value, with all other $m_{I}$ at their lowest value, then $K=2$. If the second $m_{I}$ is at its second from lowest value, with all other $m_{I}$ at their lowest values, then $K=2 S_{1}+2$ where $S_{1}$ is the maximum value of the first $S$ on card 3 . If FLAG $\neq 0$, only one width is provided (requires only I card).

There are as many of cards 5,6 , and 7 as there are species. Note that the cards 5, 6, 7 must come together. For two species the order is card 5, card 6, card 7 , card 5 , card 6 , card 7 .

If more than one spectrum is to be simulated, the data are continied from card 1. A blank card at the end of the data will terminate proceissing. The following is a sample data set which could be used to simulate hexaquncopper (II).

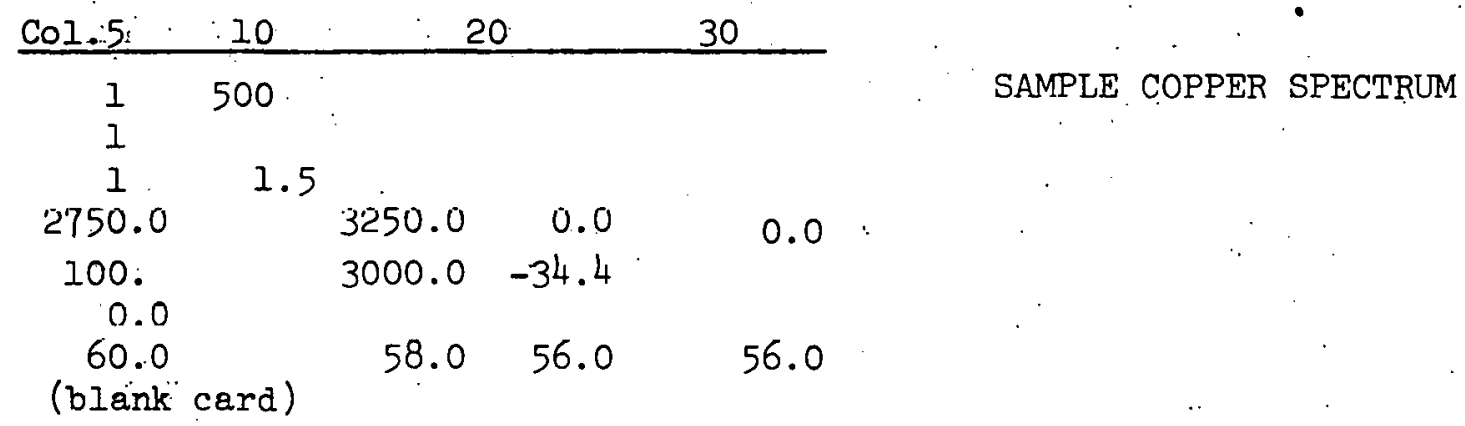


PROGRAM IHITATF(INPUT, OUTPUT, TAPE 9 , PLOT, TAPEQ9=PLOT)

C PRUGRAM FUK SIMULATING MANY SPECIES EPR SPECTKA

COMMON/SPINS/NC UUR $(10), N E(10,4), S(10,4), S M 1(10,4), S M(10), N L$ INE(4) COMMONIFSPEC/NUM, AKEA, FX(2000), FZ(2000). ,NSPECY COMMON/STIRL I/A (10,4), FINT(4),W(1600), R(1600), H(1600), JS COMMON/CEPOCL/XMIN, XMAX, YMIN, YMAX, CCXMIN, CCXMAX, CCYNIN, CCYMAX COMMON/CCFACT/FACTOK

DIMENSION COMMENT(4), HZFRI)(4)

DATAI IWOPI $=.636619772367581$ )

5 CONTINUE

C SECTIUN FLIR INPUT OF SPECTRUM PARAMETERS

READ 401. NSPECY, NUM, ( CDMMENT $(J), J=1,4)$

C TERMINATION TEST

10 CINTINUE:

READ 402, $(N C O U P(J), J=1$, NSPECY

DO $20 \mathrm{~J}=1$. NSPECY

C READ NIJ. OF HUCLEI A.VIS SPIN

$J X=N C(I U)(J)$

READ 404, (NEQ(J),J),S(J.1,J),JI=1,JX)

NLINE $(J)=.1$

DO $20 \mathrm{Jl}=1, \mathrm{JX}$

20

NLINE $(J)=(2 * \operatorname{VEQ}(J l, J) * S(J 1, J)+1, * \operatorname{NLINE}(J)$

DO $15 \mathrm{~J}=1$. NSPECY

$K P=K \nu+N L I N E(J)$

IF I KP.GT. 1600, GO TO 4

GO TO 15

4

PRINT 421, KP

GO TOS

15 CONTINUE

READ 405, HSTART, HSTOP, ALPHA, BEIA

$K P=0$

DC $25 \mathrm{~J}=1$. NSPECY

$K P=K P+1$

$J \dot{X}=\operatorname{NCOUP}(J)$

READ.405, F[NT(J), HZERO $(J),(\Lambda(J 1, J), J 1=1, J X)$

$J 2=N L I N E(J)$

READ 405, FLAG

C FLAG $=0$. IMPLIES REAU WIDTHS FOR ALL LINES

C. FLAG.NE.O ALLL LINES HAVE SAME WIDIH

IFI FLAC, 21, 24, ?]

21 REAC 405, W(KP)

CO $22 \mathrm{Jl}=1, \mathrm{~J} 2$

$K P=K P+1$

22 CONTINUE:

GJ $10 \quad 2.5$

24 CONTIINUE

$. K P I=K P+J 2$

READ 405, (W(Ji), Jl=KP, KPl)

$K P^{\prime}=K P I$

$25 \therefore$ CONIINUE

PRINT $41 \mathrm{C}$, NSPECY, NUM

PRINT 411, ALPHA, AETA, HSTART, HSTOP

PRINI 420, ( COMPENT(J), J = 1, 4.)

: 


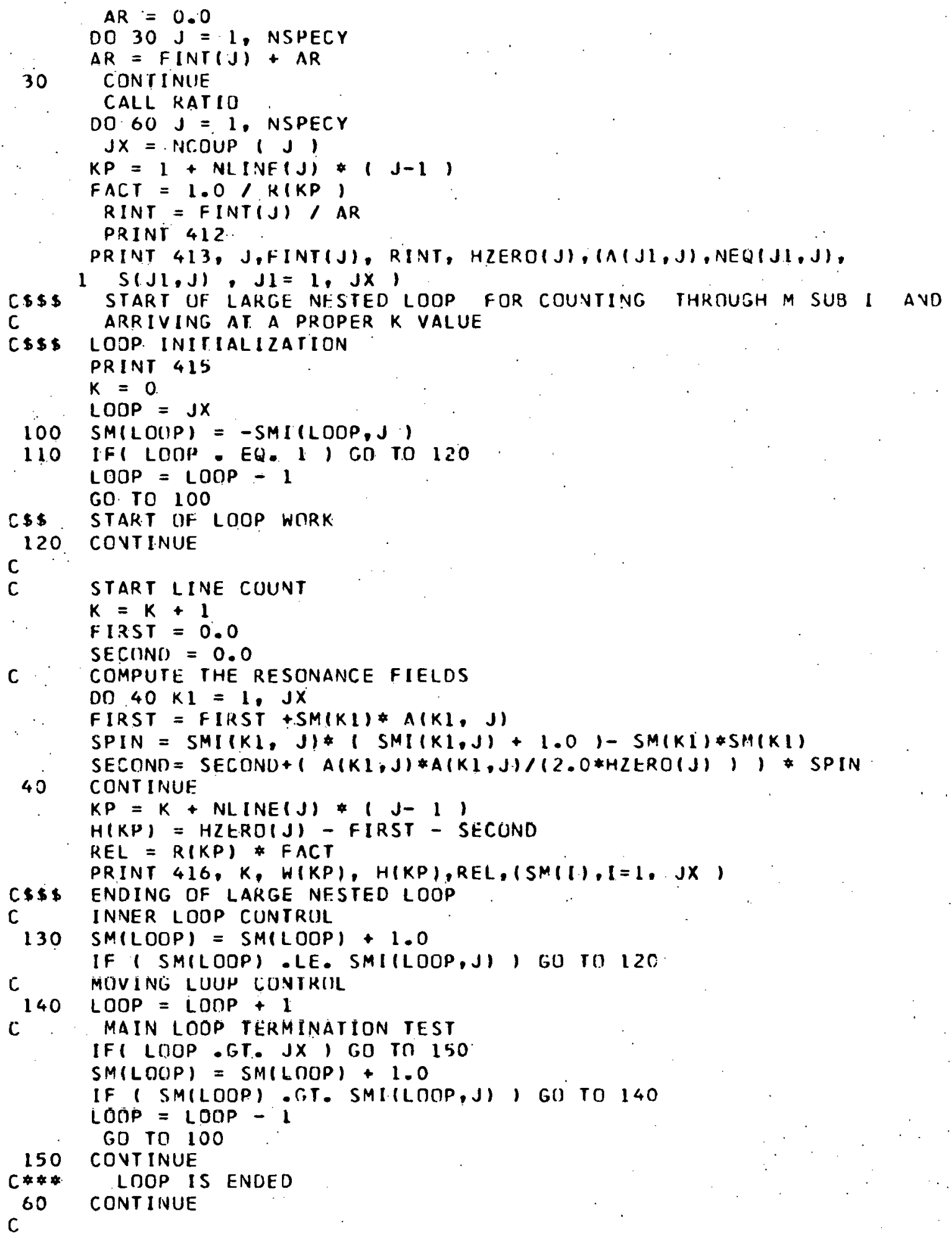




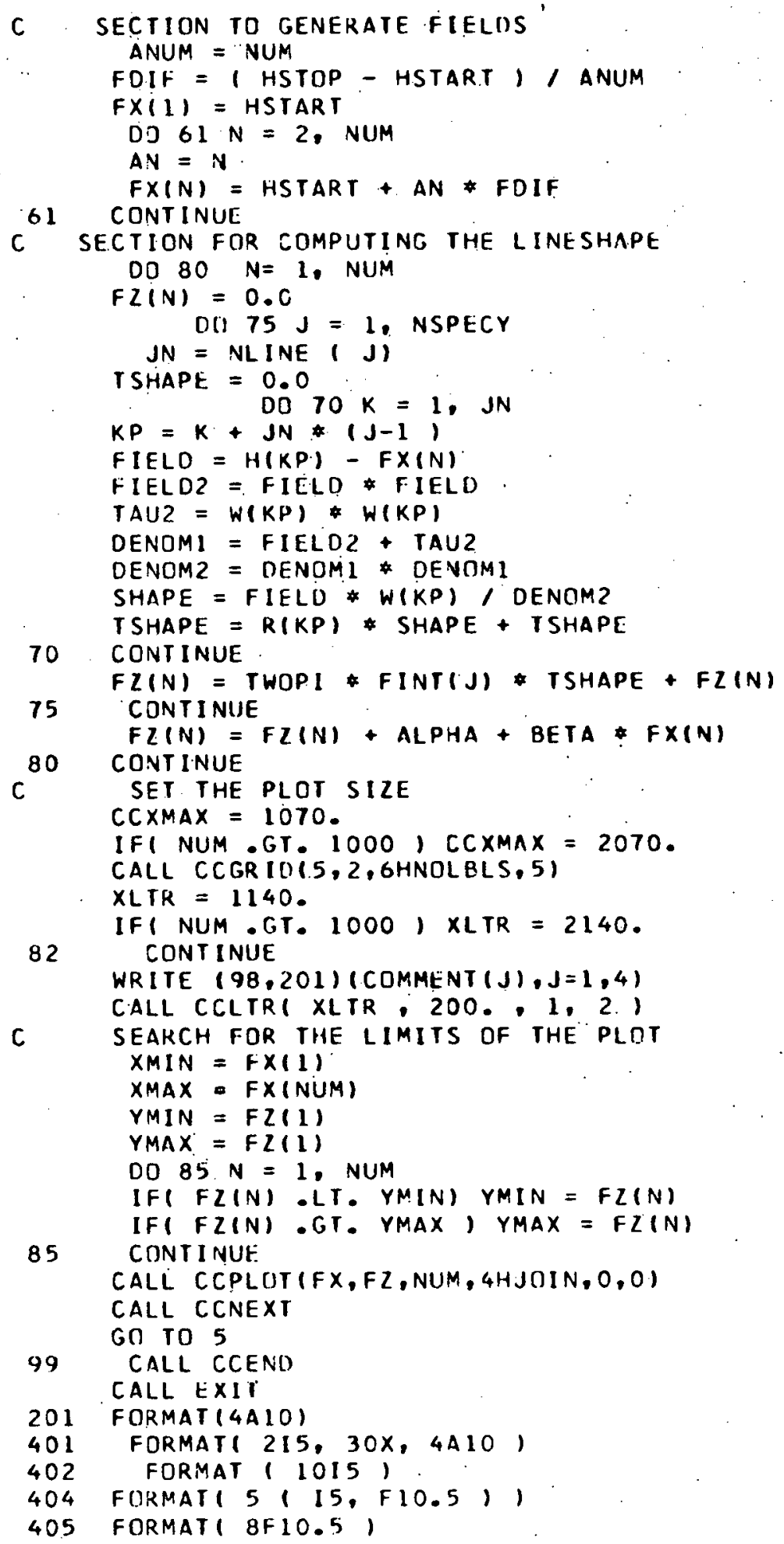


410 FORMATI IHI, 34H SIMULATION OF EPR SPECTRUM WITH $\because 15$, 1 . $9 H$ SPECIES $, 5 X, 15,8 H$ POINTS

411 FQRMATI $X$, \#ALPHA *E16.5, BETA *, E16.5, 5X, * HSTART 1 F12.3, 2X, * HSTOP * F12.31

412 FORMATI IHO, 3HNO., 5X, 1OHINTENSITY, 1OX, $10 H$ RELATIVE. $17 X ; 3 H H O, 15 X, 2 O H$ COUPLING CONSTANT

$22 X, 5 H$ Nt. $0,5 X, 4 H S I$

413 FQRMATIX, 12,5x,F10,3,10X,F7,3,5x,F10,3,15X,F10,3,10X,15,5X, 1 F5.1/ (65X,F10.3, 10X,I5. 5X,F5.1),

415 FORMAT (IHO, $10 H$ COMPONENT $, 1 X, 12 H$ LINEWIOTH .IX, 1 I IHH LIVE CENTER. 1

416 FORMAIIX,15,F10.3,5X,F10.3,F8.1.1012X,F4.1),

420 FOKMATI 1 HO, 30X, 4A10, $1 /$

421 FORMATI 1 HO, 17, 32H EXCEEDS NO. OF POSSIRLE LINES END 


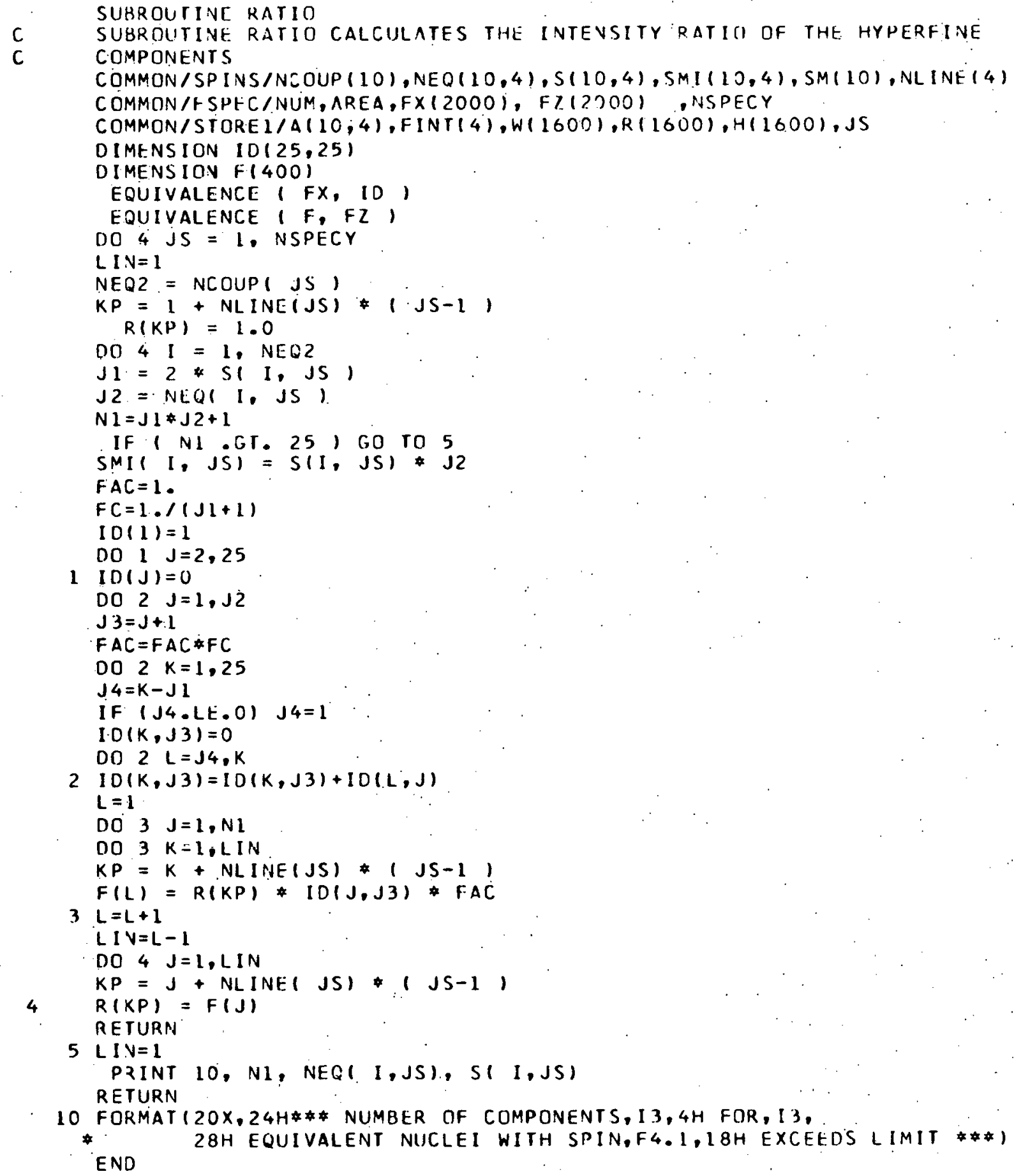




\section{2. $\operatorname{VAESR}^{*}$}

VAESR is used to simulate polycrystalline or glass spectra for transition metal complexes with spin $] / 2$ and an axial spin Hamiltonian. The method used is due to Vanngard and Aasa (1962). The input essentially consists of the microwave frequency, and the spin Hamiltonian parameters. The main output is a computer drawn plot of the spectrum. Mellival

The lineshape used is given by

$$
I(H)=g_{\perp}^{2} \frac{1}{\delta(2 I+1)} \quad \int_{0}^{1} S^{\prime}\left(H-H^{\circ}\right)\left[\left(g_{\|} / g\right)^{2}+1\right] d z
$$

where $z=\cos \theta, \theta$ is the angle between the molecular axis and the applied magnetic fields, $S^{\prime}$ a shape function. For a Lorentz lineshape, the shape function is given by

$$
S^{\prime}(x)=\frac{2}{\pi} \frac{1}{\alpha^{3}} \quad x\left[1+(x / \alpha)^{2}\right]^{-2}, \alpha=(\sqrt{3} / 2) \Delta H
$$

where $\Delta H$ is the peak-to-peak derivative width. The resonance field is given by

$$
\begin{aligned}
& \mathrm{H}^{\circ}=\mathrm{HO}_{\mathrm{O}}-\mathrm{KM}_{\mathrm{I}} / \mathrm{gB}-(1 / 4 \mathrm{Ho})(\mathrm{gB})^{-2} \mathrm{~A}_{\perp}{ }^{2}\left(\mathrm{~A}_{\mathrm{j}}{ }^{2}+\mathrm{K}^{2}\right) \mathrm{K}^{-2}\left[\mathrm{I}(\mathrm{I}+\mathrm{I})-\mathrm{m}_{\mathrm{I}}^{2}\right] \\
& -\left(1 / 2 \mathrm{H}_{0}\right)(\mathrm{g} \beta)^{-2}\left(\mathrm{~A}_{\|}{ }^{2}-\mathrm{A}_{\perp}^{2}\right) \mathrm{K}^{-2} \mathrm{~g}_{\|}^{2} \cdot \mathrm{g}_{\perp}^{2} \mathrm{~g}^{-4} \mathrm{z}^{2}\left(1-\mathrm{z}^{2}\right) \mathrm{m}_{\mathrm{I}}^{2} \\
& \mathrm{~g}^{2}=\mathrm{g}_{1}^{2}+\left(\mathrm{g}_{\|}^{2}-\mathrm{g}_{1}^{2}\right) \mathrm{z}^{2} \cdot \mathrm{K}_{\mathrm{g}}^{2}=A_{\|}^{2} \mathrm{~g}_{\|}^{2} \mathrm{z}^{2}+\mathrm{A}_{1}^{2} \mathrm{~g}_{1}^{2}\left(1-\mathrm{z}^{2}\right) \\
& \text { Hò }=h \nu / g \beta
\end{aligned}
$$

A variable Iinewiath is permitted for each hyperfine component, and the lineshape is summed over the hyperfine components. The integral is evaluated using Simpson's rule.

The original version of this program was supplied by $W$. Burton Lewis. The program has been considerably altered. 
INPUT DATA

Card 1 (Format I.5, 6AIO)

MCI the number of hyperfine components (maximum of 8 ).

LABEL any title information for the plot

Card 2 (Format 8F10.0)

$X M(I)$ the $m_{I}$ values for the hyperfine components (in order)

Card 3 (Format I5, 3Fi0.0)

N The number of points over which the Simpson's Rule integration is to be performed; must be odd.

$\mathrm{DZ}$ The size of the interval for the integration. May range from. 0 to $1.0(\mathrm{DZ}=\cos \theta)$. For $\mathrm{N}=201, \mathrm{DZ}=.005$.

HT The lowest point in the magnetic field for the calculation and plotting of the spectrum.

HFNAL The highest point in the magnetic field.

Card 4 (Format 8F10.0)

A

Al in gauss

B

$\Lambda_{1}$ in gauss

C

leave blank (not used)

GPL $\quad \because \quad g_{\|}$

GPR. $\quad g_{1}$

$X I \quad I$, the nuclear spin (eg. for $I=3 / 2, X I=1.5$ )

$\mathrm{SH} \quad \mathrm{h \nu} / \mathrm{B}$ (for $\nu=9.15 \mathrm{GHz}, \mathrm{SH}=6537.565$ )

CMP The value by which the magnetic field is to be stepped for calculations. The intensity of the spectrum is calculated at each point, $n$, where the value of the field at the nth point is $H T+n * C M P$. 
Card 5 (Format 8F10.0)

DH(I) The linewidths for each of the hyperfine components

Card 6 (Fomat 8F10.0)

ZERO Vertical distance in inches from the bottom of the plot to the baseline. May be from 0 to 10 inches. This defines the baseline for the piot...

SIZE Length of the largest peak in inrhes from the baseline (ZERO). Note: If the peak is negative going; then SIZE is negative. Cards are repeated from card 1 to isimulate additional spectra. A blank card at the end of the data is used to terminate the program. 


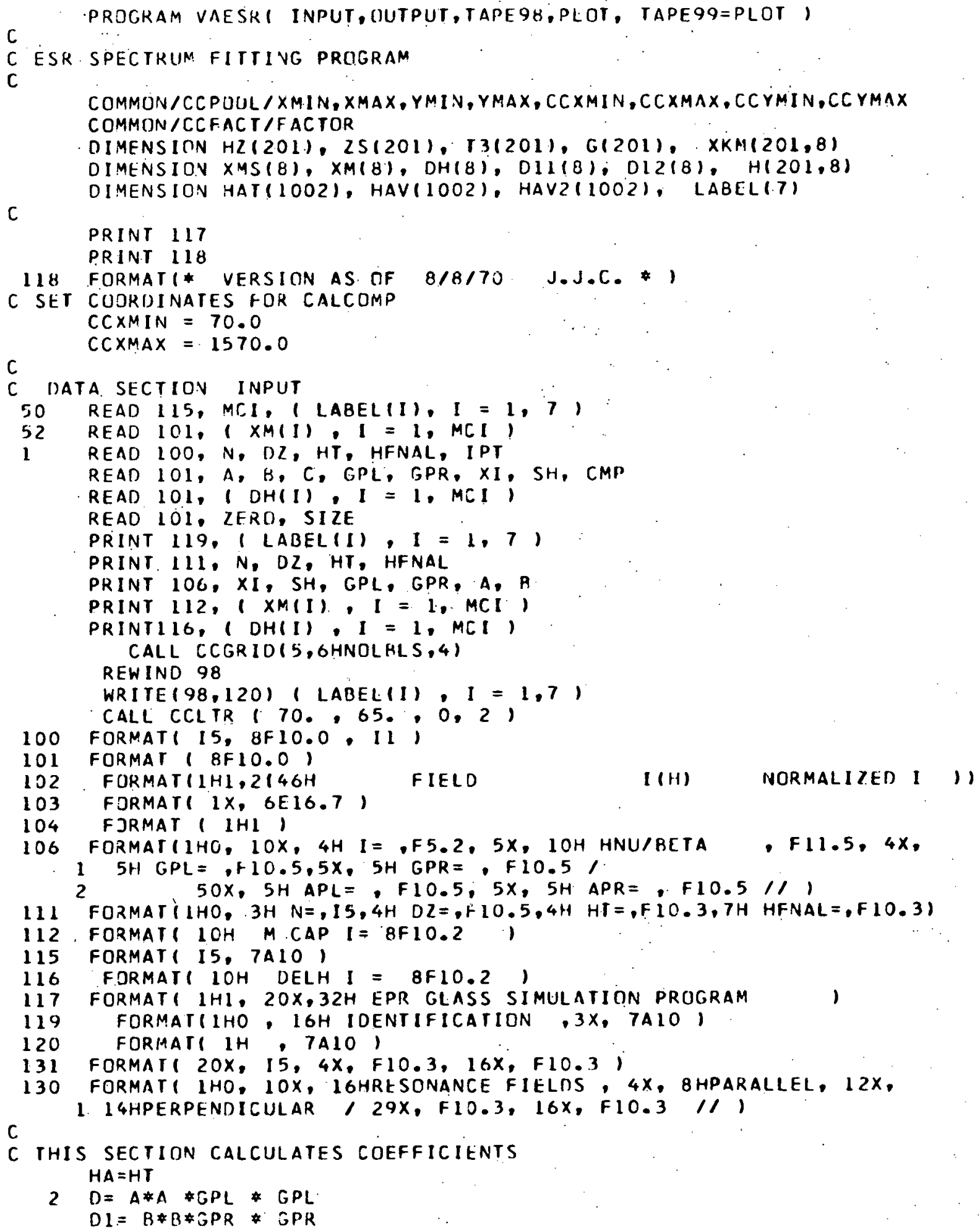




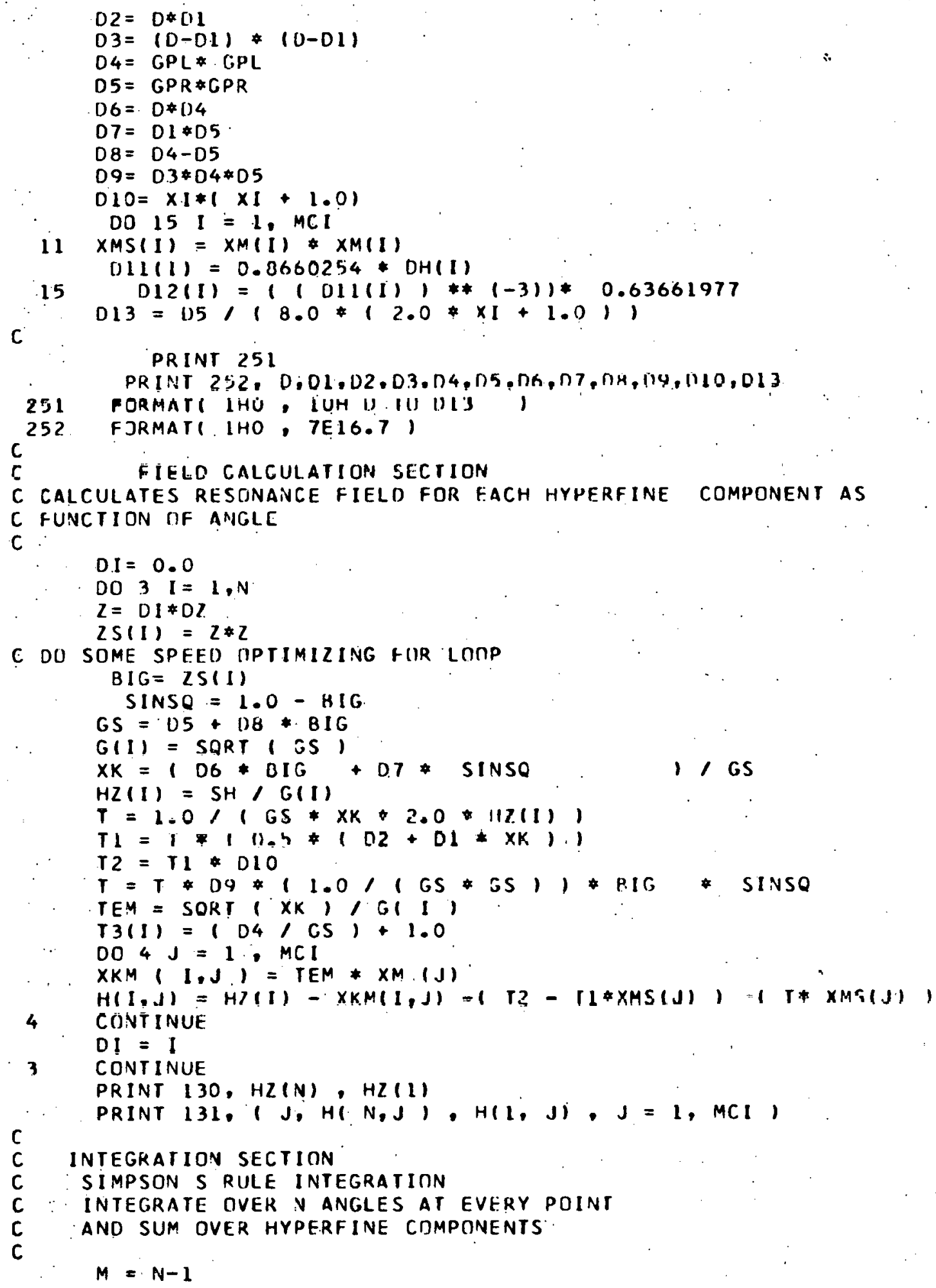


$\operatorname{CON}=6.0$

$B I G=0.0$

20 DO $21 \mathrm{~L}=1,1002$

$A B E=L$

SMKS $=0.0$

SMJS $=0.0$

DO $23 \mathrm{~J}=1, \mathrm{MCI}$

$X=H A-H(1, J)$

C. USE FSIZE FOR TEMPORARY FOR SPEED

FSILE $=011(\mathrm{~J})$

$Y=(1 \cdot x / F S \mid Z E) * 21+1.0$

$Y=1: 0 /(Y * Y)$

TEMP $=012(\mathrm{~J})$

SMINT $=$ T3(1) TEMP $* x * r$

$T E M=4.0$

DO $24 . I=2 . M$

$X=H A-H(I, J)$

$Y=(1 X / F S I Z E) * 21+1.0$

$Y=1.0 /(Y * Y)$

SUM $=T 3(1)$ TEMP $* X * Y$

SMINT = SMINT + SUM*TEM

TEM = CON - TEM

24 CONTINUE

$X=H A-H(N, J)$

$Y=(\mid X / F S I Z E) * 2)+1.0$

$Y=1.0 /(Y * Y)$

SUM $=I 3(N) *$ TEMP $* X * Y$

SMINT $=$ I SMINT + SUM, 1 DL/3.0

C

SMJS = SMJS + SMINT

23 CONTINUE

SMKS $=$ SMKS + SMJS

c

22 CONTINUUE

$$
\text { HAV (L) = SMKS* D.13 }
$$

C START TO FINU MAXIMUM FOR SCALING TEMP = ABS ( HAV (L) )

IFI TEMP - BIG, $30,30,32$

32 BIG = TEMP

30 CONTINUE.

$\operatorname{HAT}(L)=H A$

$H A=H J+C M P * A B C$

IFI HA-HFNAL I 21, 21, 9

21. COVTINUF

$9 M=A B C$

C SECTION TO SCALE SPECTRUM FOR PLOTTING

$X M I N=H T$

$X M \triangle X=H F N \wedge L$

$Y M I N=-1000$

YMAX $=1000 . *(1.0-2 E R O, 10$.$) (10.), 2ERO)$

IFI SILE, $40,40,41$

40 FSIZE $=1$ SIZL, ZERO) YMIN

GO TO 42

41. FSILF $=1$ SILE / 10.0 - ZERII.) * YMAX 


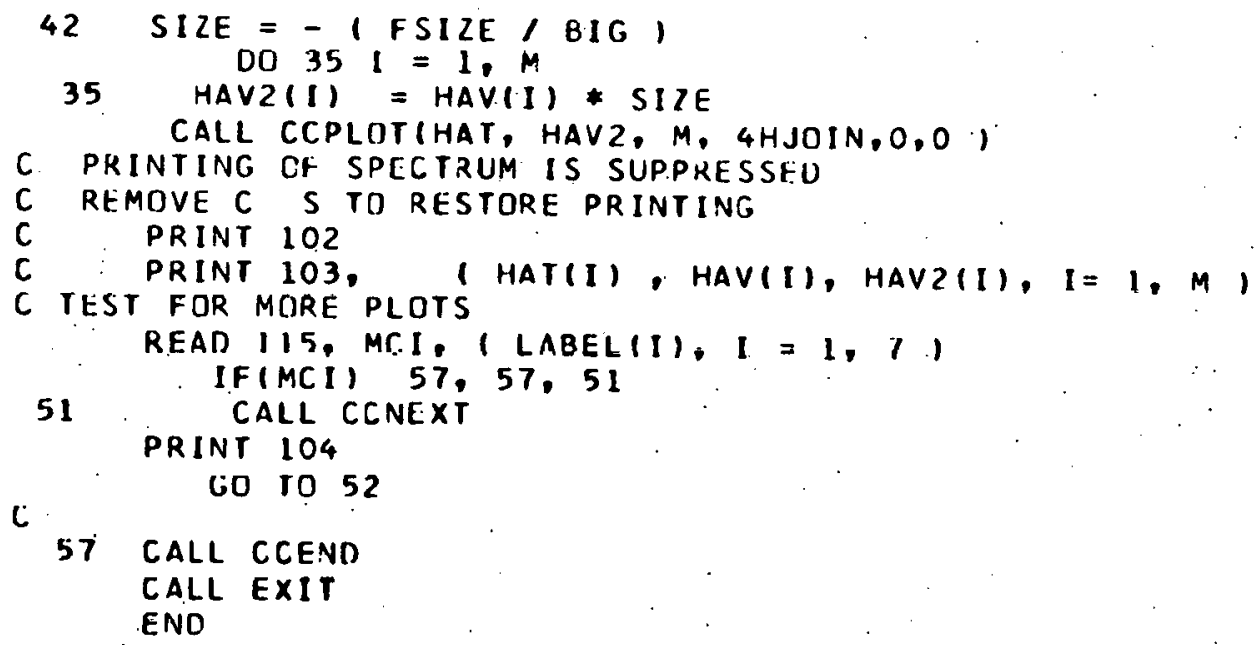




\section{T $_{1}$ Analysis Program - LOGLINE}

The LOGLINE program was written to operate in connection with the BASIC AVERAGER program supplied by the Digital Equipment Corp. with the LAB-8/I computer system. It is used to analyze the exponential recovery curves obtained in pulse-saturation-recovery experiments. It can, however, analyze any exponential recovery curve. The LOGLINE program can punch the experimental data acquired. by the BASIC AVERAGER on the high speed punch; read in previously punched data for analysis; display the data on a storage display scope; or analyze the recovery curve for the time constant The program operates under the control from the teletype; the analysis is performed interactively, on-line, with the operator using the teletype to control the analysis, and the display oscilloscope to observe the results.

LOADING

The following program tapes must be loaded in order using the BINDARY LOADER

1. Lab-8 Basic Control. Tape DEC̈-LB-T21A-PB

2. Lab-8 Basic Averager DEC-LB-U2IA-PB

3. LOGLINE program tape

4.. Floating Point Pkg. \#3 DEC-08-YQ3A-PB without EAE or DIGITAL-8-25-F-BIN with EAE

At the end of the loading operation the Control tape and the Basic Averager are in Field 0 . The main part of the LOGLINE program and the floating point package are in Field 1 . Note that the operation of these programs requires a PDP-8/I equipped with $8 \mathrm{~K}$ of memory, a high 
speed paper tape reader and punch, a DF32 disk, and an AXO8 laboratory peripheral.

OPERATION

The programs are first. loaded with the binary loader. The program is started at address $07603_{8}$. The basic averager is used to acquire a recovery curve (The operation of the Basic Averager is discussed in the DEC manual, DEC-LB-T2OA-B): The inputs to the AX08 should be adjusted so that the recovery curve is all negative; that is, the recovery curve grows from minus infinity to zero. The reason for this is that the data is converted to an exponential decay curve for the purpose of the logarithmic analysis simply by making all the data positive.

After the data has been acquired the LOGLINE program is entered from the basic averager by typing CTRL/P. LOGLINE types " $R$ " to request the sample rate. The sample rate should be typed in some appropriate unit such as miliseconds. The sample rate is the time between data points and defines the time scale for the experiment. After the sample rate is typed the user may either punch the data or proceed to analyze the data. If the data is to be punched, CTRL/N is typed. LOGLINE will ask FIRST and LAST and the starting and ending channel to be punched should be provided. Note that the high speed punch should now be turned on. The user now types CTRL/P to punch the data. After the data is punched, the punch is turned off and the user may proceed to analyze the data. The first step in the data anslysis: is to type the AIT MODE key. This causes the Basic Averager to be stored on the disk, thus freeing FIELD 0 for data manipulations. LOGLINE will reply with the message CORE SWAPPED after the operation is completed. At this time the baseline 
should be subtracted from the data. Type CTRL/N to redefine FIRST and LAST to the channels near the end of the recovery, for example. FIRST $=960$ and LAST=990. Now type CTRL/B to compute the baseline from the average of the data from FIRST to LAST. LOGLINE will reply with the value of the baseline. Now type CTRL/A to subtract the baseline. New values of FIRST and LAST are requested for the baseline subtraction. (Note: all operations on the data are performed with FIRST and LAST as limits).

At this time the data may be examined on the dispiay scope by typing CTRL/D to display the data from FIRST to LAST The logarithm of the data is displayed by CTRL/F. The display may be expanded or contracted by tiping as manys $X^{\prime} s$ or C's as are required.

The data are now analyzed for the time constants. CTRL/N is typed and the limits are redefined for the next command. CTRL/V is typed to command LOGLINE to fit the logarithmic data from FIRST to LAST with a straight line. At the end of this operation LOGLINE types the constants of the stralght line and types the relaxation time. (A is the intercept and $B$ is the slope of the straight line). The theoretical curve can be compared with the experimental curve by typing CTRL/L to display the theoretical logarithm. If the fit is bad, the limits should be changed with CTRL/N and the fit performed again with CTRL/V. CTRL/N may be used at any time to change limits of fitting or of displaying data. If there are additional time constants present in the data, the theoretical logarithm should now be stripped from the data by typing CTRL/S. The analysis can then proceed with a CTRL/N, CTRL/V combination as before. These operations are repeated to extract all the time constanta. The progress of the fit is checked at all times with the display commands. 
At the end of the processing the BASIC AVERAGER is reentered by typing CTRL/Q. At this point LOGLINE reads the basic averager into field 0 from the disk and then jumps to the basic averager. Note that if a mistake has been made in analyzing the above data, the data can be restored by typing CTRL/Q to return to the averager and then CTRL/P to return to LOGLINE with the original data. The analysis may then be repeated from the beginning.

To analyze dula which has been previougly punched, the LOGLINE program is first entered from the averager with CTRL/P. The data tape is placed in the high speed reader and CTRL/R is typed. LOGLINE will read the data tape. The analysis may now proceed as if LUGLINE hau jusl been entered from with averager with data. 
LOGLINE COMAANDS

CTRL/A
CTRL/B
CTRL/D
CTRL/E

strip baseline from data between FIRST and .LAST

compute baseline from between FIRST and LAST

display data from FIRST to IAST

examine the data in the channel indjcated by FIRST LOGLINE will type the contents of the (FIRST) channel. The user now has 3 options:

space: after typing a space the user can type a new value for the channel.

line feed: typing the line feed causes FIRST to be changed to FIRST+1 and this channel is typed

return: typing a carriage return terminates the CTRL/E command.

CTRL/F

display the logarithm of the data from FIRST to LAST

CTRL/H halt current operation. Oniy operates during a CTRL/N or during a CTRL/P operation. Note that a CTRL/N occurs during a CTRL/A and a C'RRL/S operation as part of their function. This comand permits some mistakes during operation to be corrected.

CIRL/L display theoretical logarithm from FIRST to LAST. In the special case of a CTRL/T command, this command is used to terminate the CTRL/T command.

CTRL/N

define new values of FIRST and IAST. The CTRL/N operation may be halted by CTRL/H. The values of FIRST and LAST after CTRL/H are uncertain.

CTRL/P punch data from FIRST to LAST on high speed punch. Must be used before the ALT MODE conmand.

CTRL/Q quit. return from LOGLINE to the basic averager.

CTRL/R read a previously punched data tape. Must be used before ALT MODE.

CTRL/S. "strip the theoretical logarithm from the data from FIRST to LAST.

CTRL/T. title. All information typed is echoed and ignored until CTRL/L is typed. This command is useful to provide descriptive information on the output during processing.

CTRL/V

Fit a straight line to the logaritha of the data from FIRST to IAST.

AL'L' MUUE'

swap the basic averager from field 0 to the disk; make the data positive and convert it from integer format to floating point.

$\mathrm{X}$

expand the display by factor of two

C

contract the display by factor of two

CINHL/K

("II'ILI. /W

CI'RL/U

These commands are not presently defined. They are provided for ruture expansion of the program. 


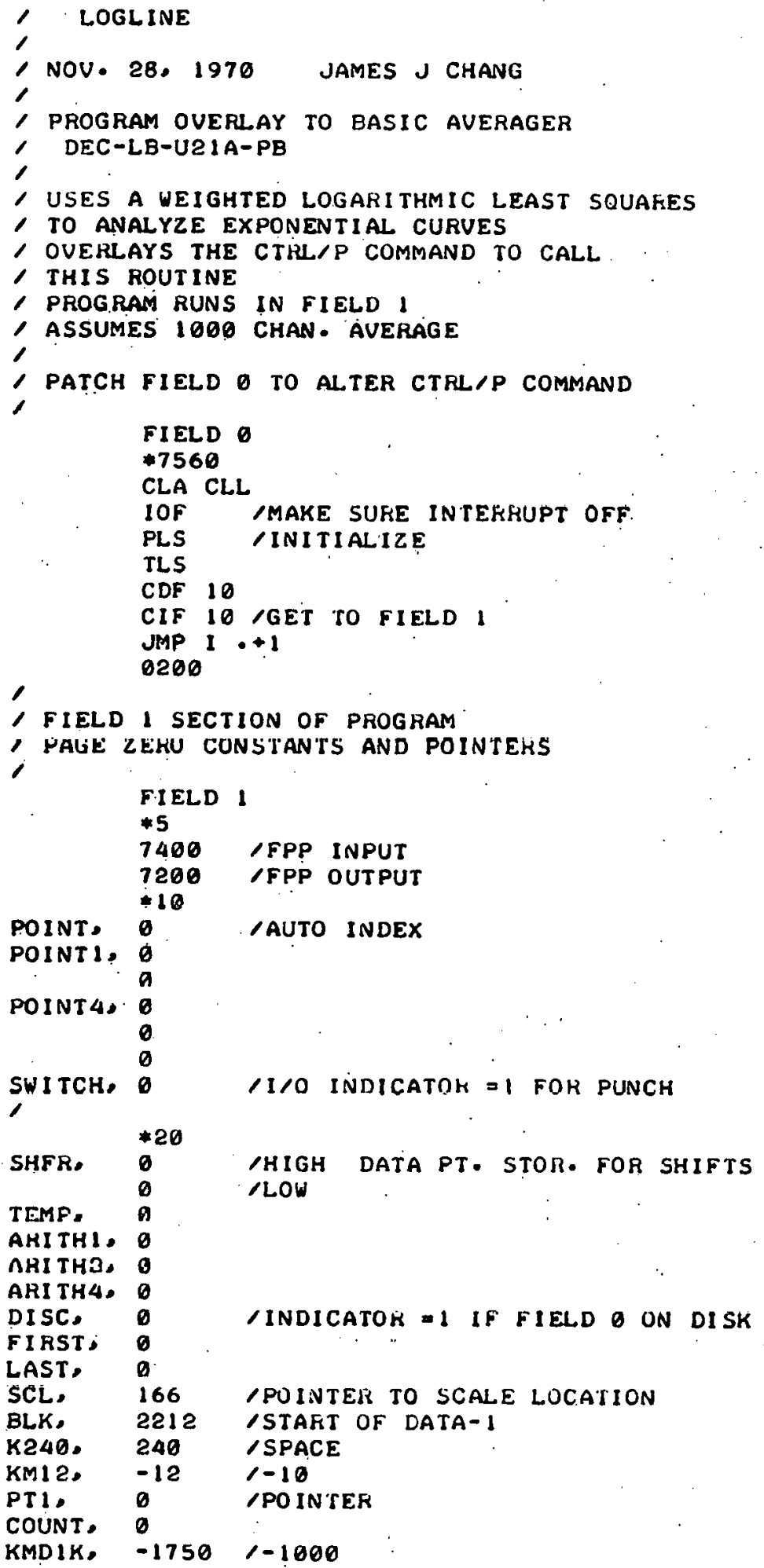




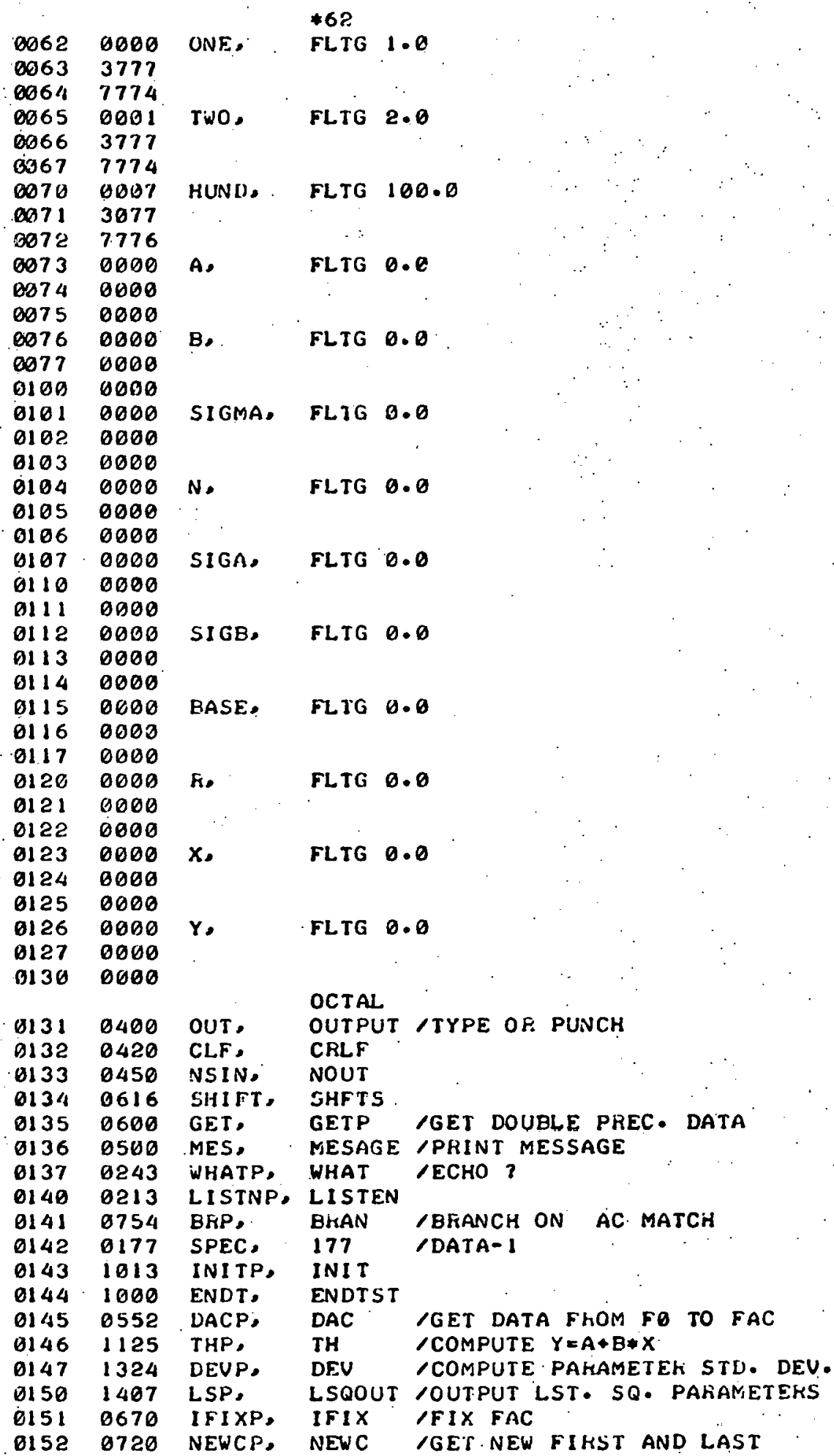




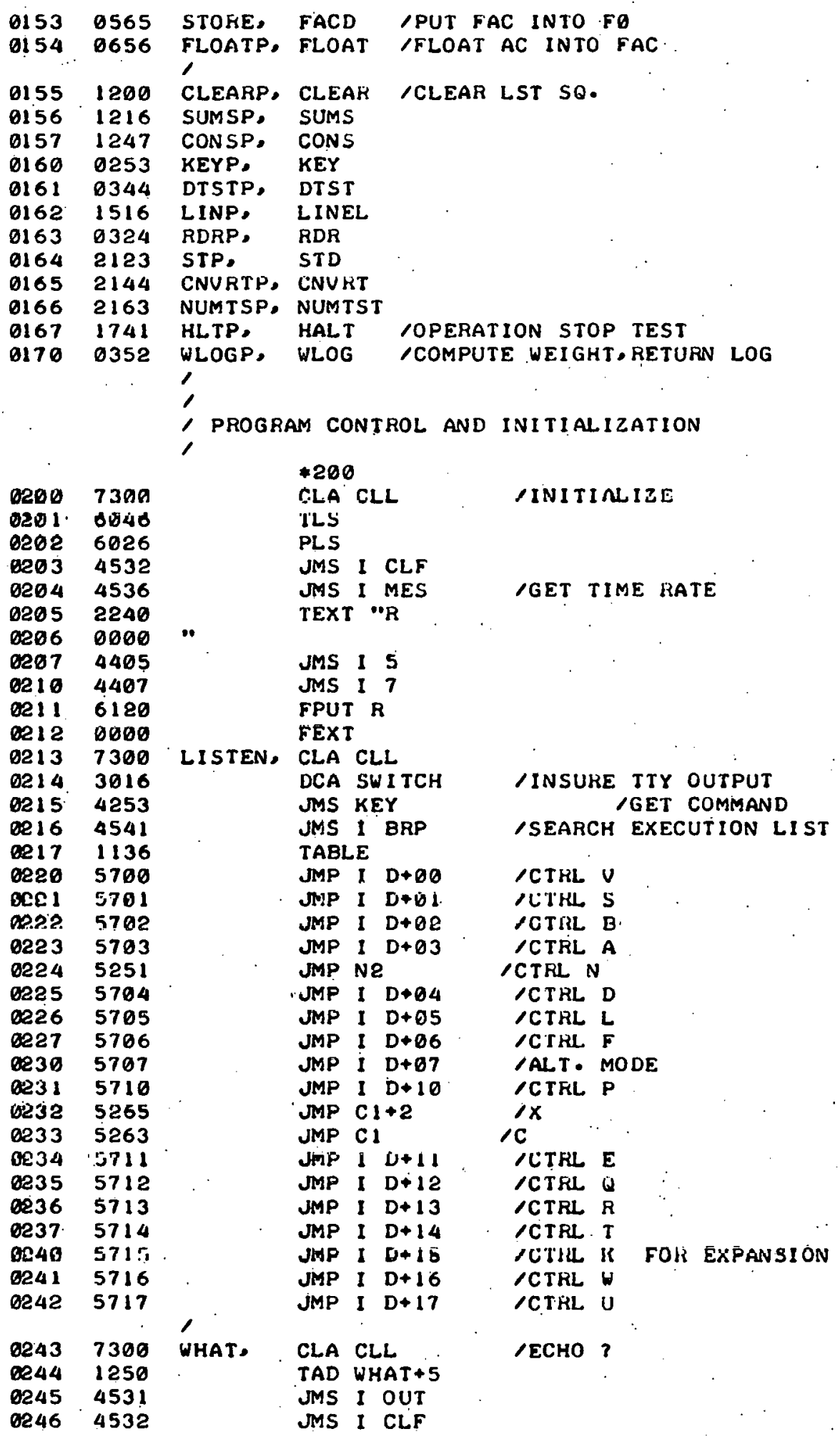




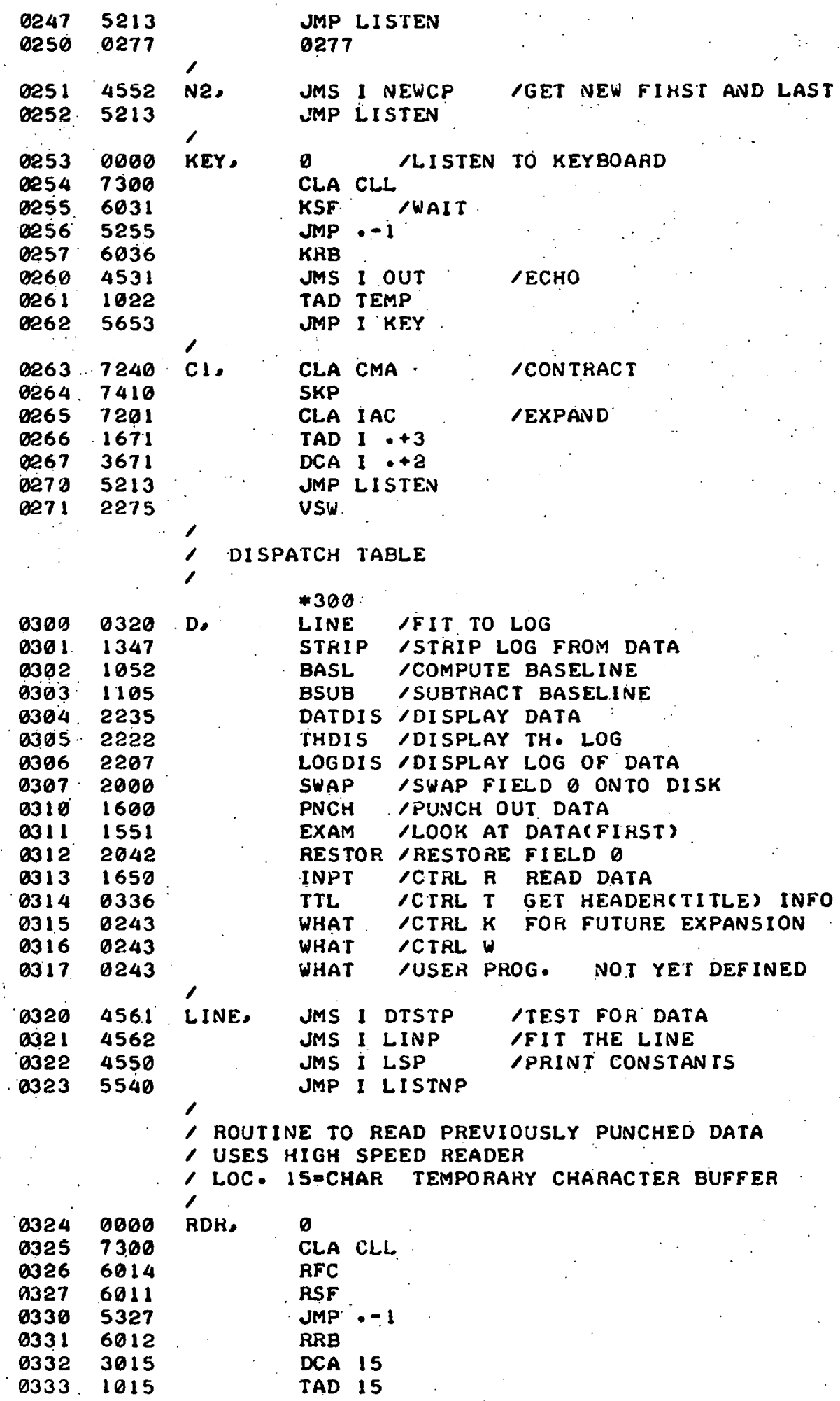




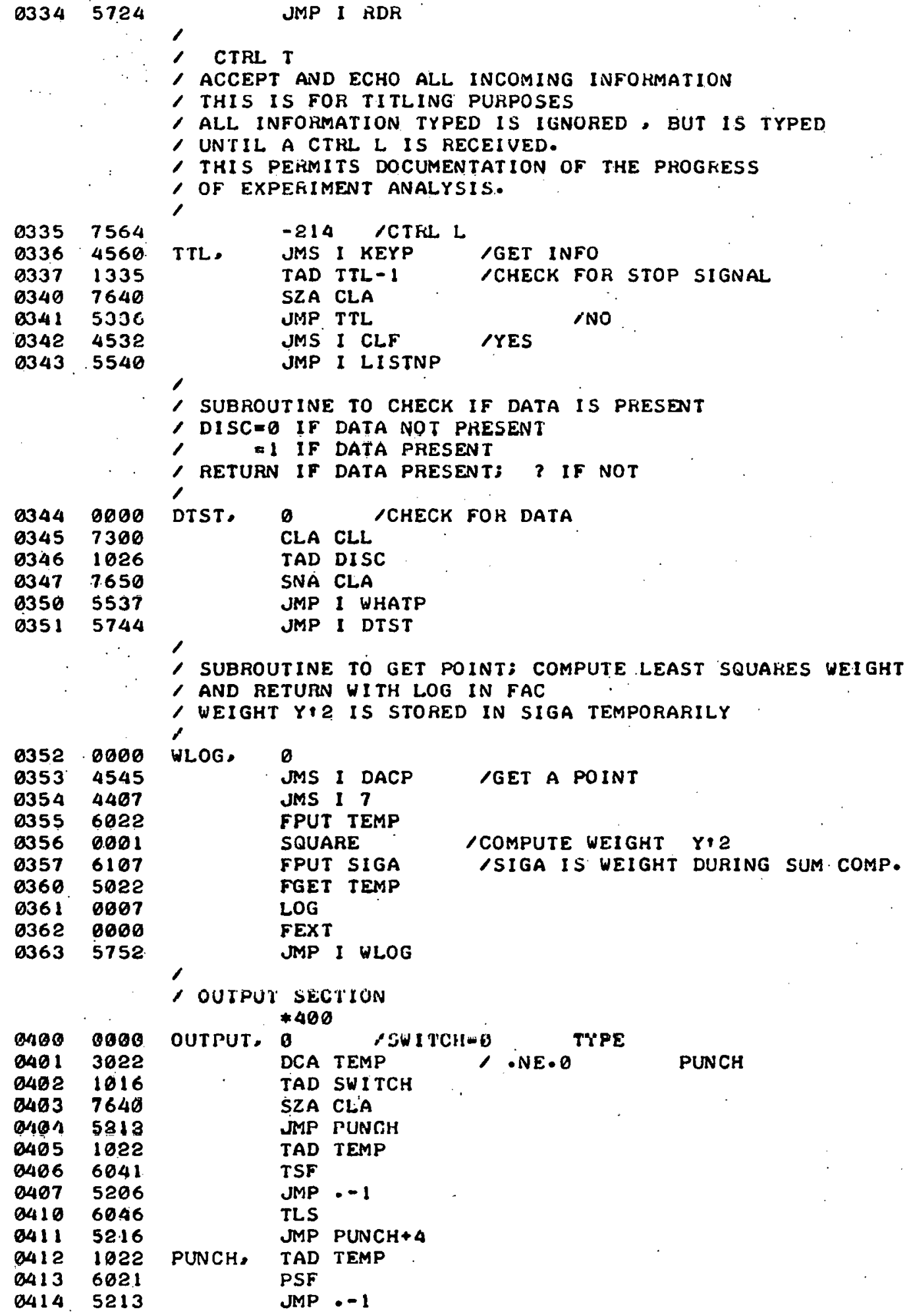




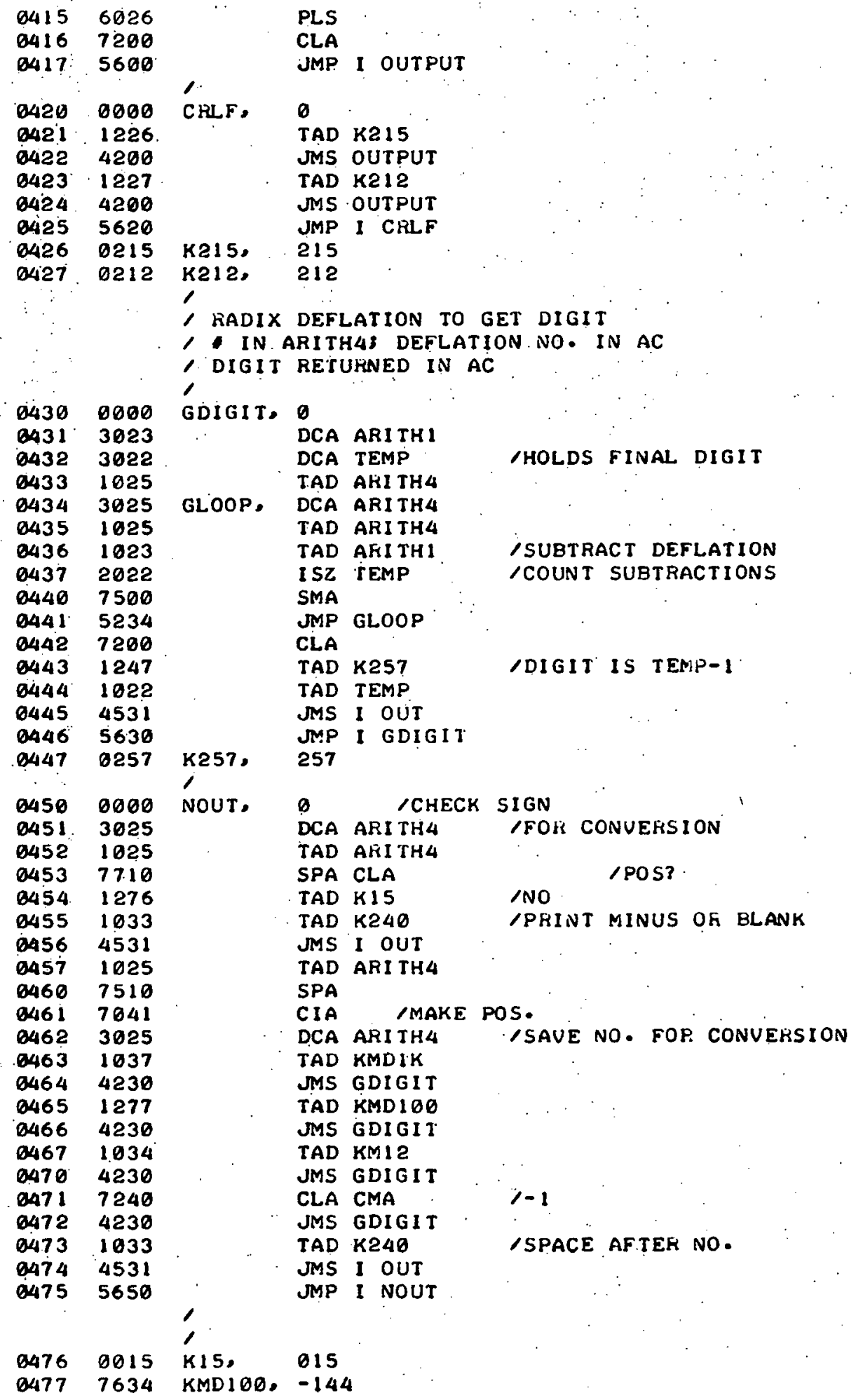


1

1 MODIFIED VERSION OF

/DIGITAL $8-18-U$

/MESSAGE TYPE-OUT

ICALL WITH A JMS MESAGE

/WITH DATA FOLLOWING

/RETURN FOLLOWING END OF MESSAGE

$\angle C O D E(D O)$

/ USES NOUT FOF TEMPORARY STOKAGE

- CANNOT BE PRINTING NO. AT SAME TIME

IPRINTING MESSAGE

050

I

MESAGE,

05017240

$0502 \quad 1300$

$0503 \quad 3010$

05041410

05053250

$0506: 1250$

$0507 \quad 7012$

05107012

05117012

$0512 \quad 4316$

0513.1250

$0514 \quad 4316$

$0515 \quad 5304$

05160000

$0517 \quad 0344$

65207450

05215410

$6522 \quad 1345$

05237500

$6524 \quad 5327$

05251346

$0526 \quad 5342$

$0527 \quad 1347$

$0530 \quad 7440$

05315334

$6532 \quad 1227$

0533 5342.

05341350

05357448

05365341

$0537 \quad 1226$

$0540 \quad 5342$

0541.1351

05424200

09435716

$05114 \quad 0077$

$0545 \quad 7740$

$0546 \quad 8340$

$0547 \quad 7775$

$0550 \quad 7776$

05510245

TYPECH.

\section{CLA CMA}

TAD MESAGE

DCA 10

TAD I 10

DCA NOUT

TAD NOUT

RTR

RTR

RTK

JMS TYPECH

TAD NOUT

JMS TYPECH

JMP MESAGE + 4

0

AND MASK77

SNA

JMP I 10

TAD $M 40$

SMA

JMP +3

TAD C 340

JMP MTP

TAD M3

SZA

JMP $\bullet+3$

TAD KEIR

IMP MTP.

TAD M2

$S Z A$

$J M P .+3$

TAD K215

JMP MTP

TAD C245

MTP, JMS OUTPUT

/CONSTANTS

MASK 7.7, 77

M40, -40

C340, $\quad 340$

M3. -3

Me, $-\varepsilon$

C245. 245

/ GET FIELD U DATA INTO FAC

1

05520900

05537206

DAC, $\quad 0$

C.A
/SET $C(A C)=-1$

$\angle A D D$ LOCATION

$\angle$ AUTO-INDEX REGISTER

/FE'TCH HIRST WOKD

I SAVE IT

/ROTATE 6 BITS RIGHT

ITYPE IT

GET DATA AGAIN

I TYPE RIGHT HALF

ICONTINUE

/TYPE CHARACTER IN C(AC)6-11

IIS IT END OF MESSSAGE?

CYES: EXIT

ISUBTRACT 40

$1<40$ ?

1 NO

$\angle$ YESR ADD 300

1 TO CODES $<40$

ISUBTRACT 3

IIS IT ZERO?

1 NO

/YES: CODE 43 IS

/LINE-FEED ( 312$)$

/SUBTKACT 2

IIS IT ZERO?

INO

/YES: CODE 45 IS

/ CARFIAGE-FETURN (215)

ITYPE MESSAGE 


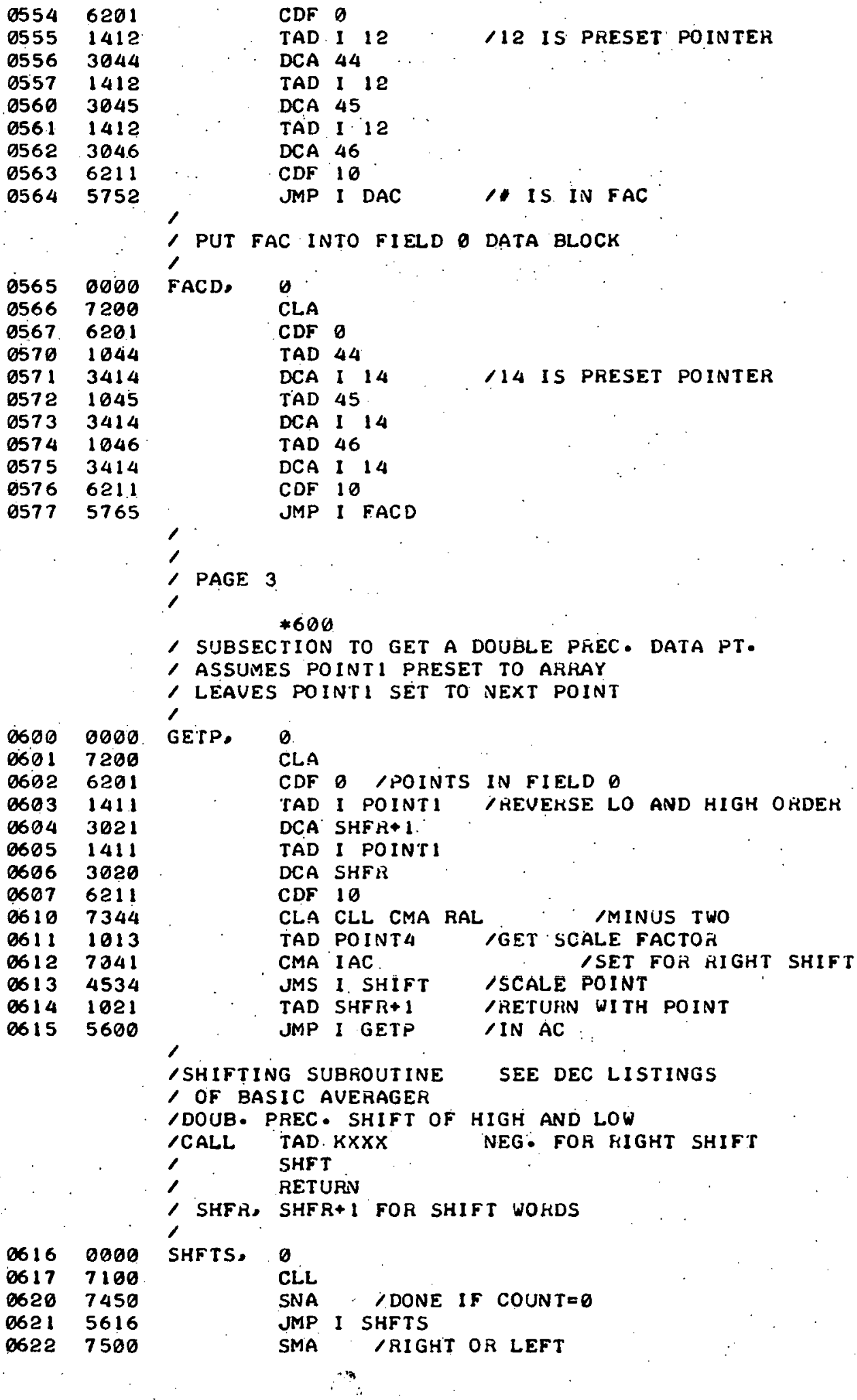




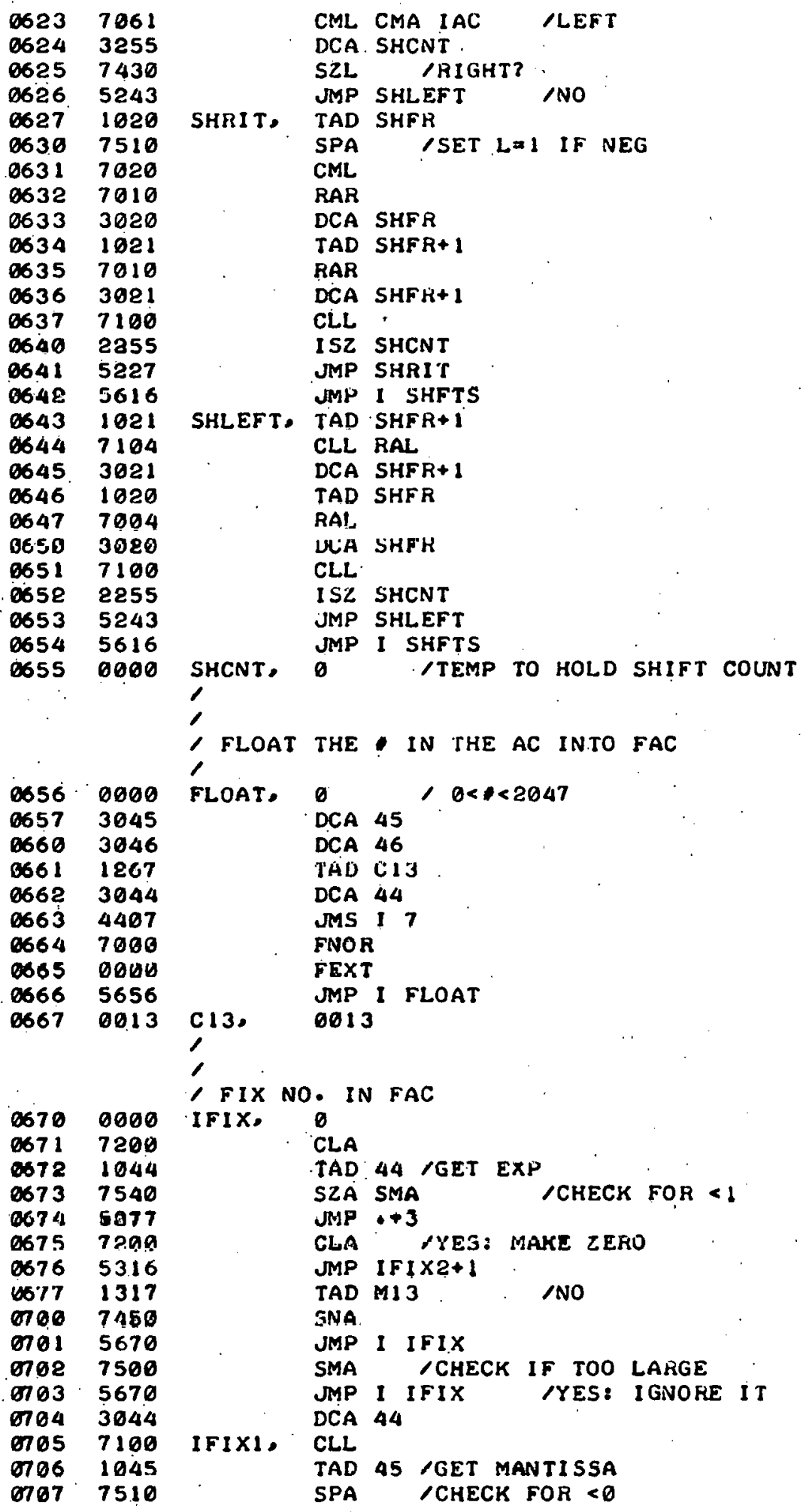




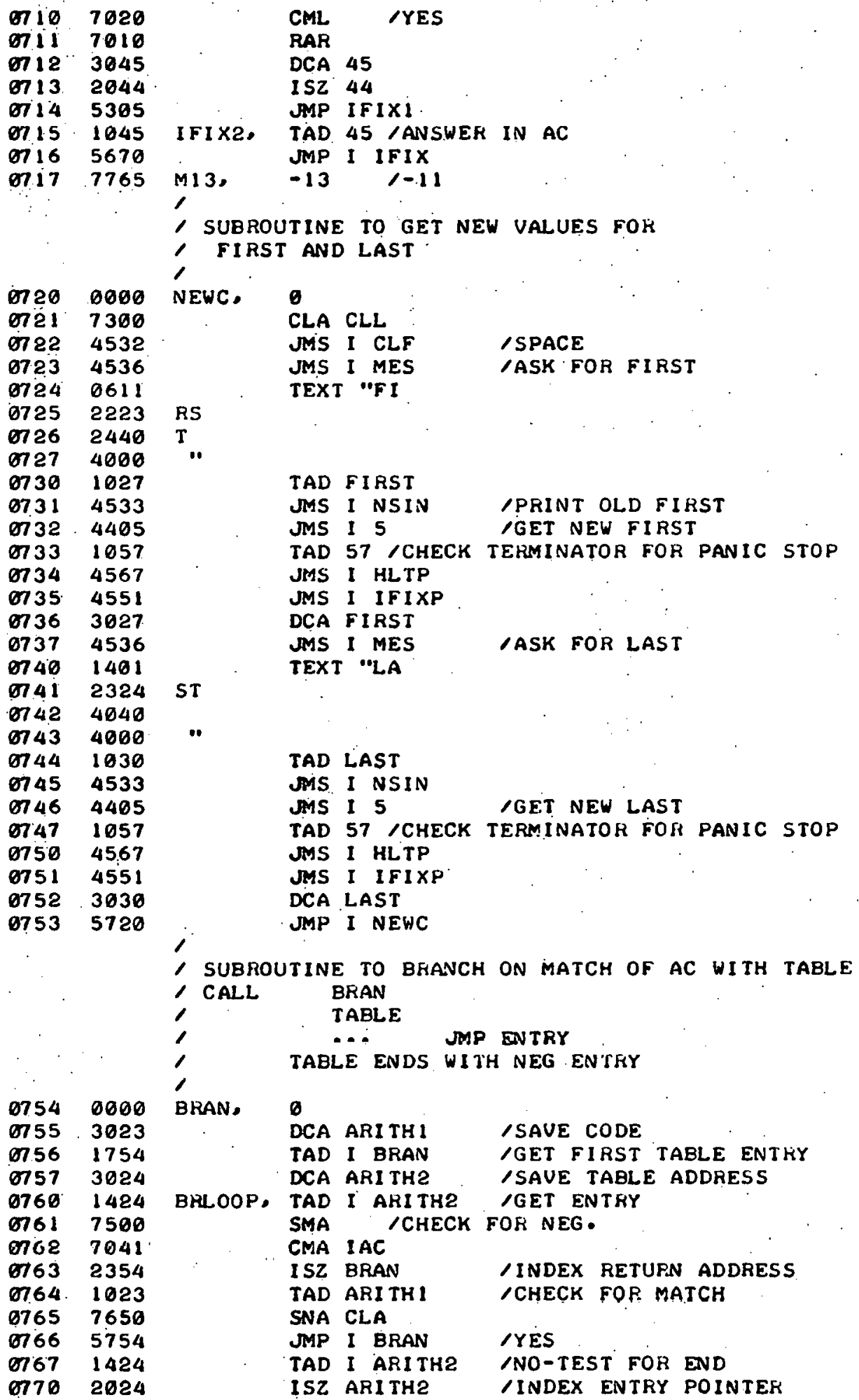




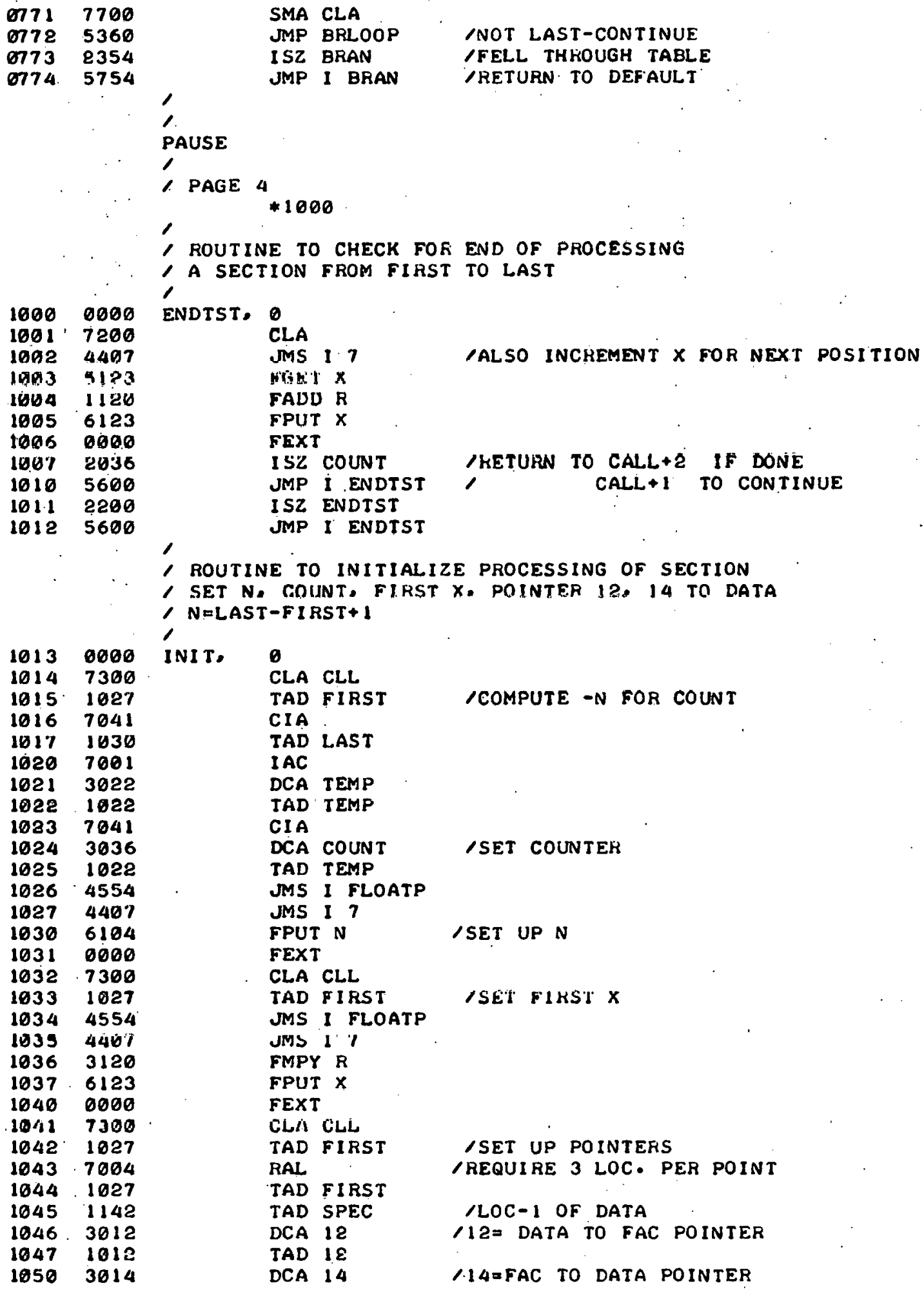




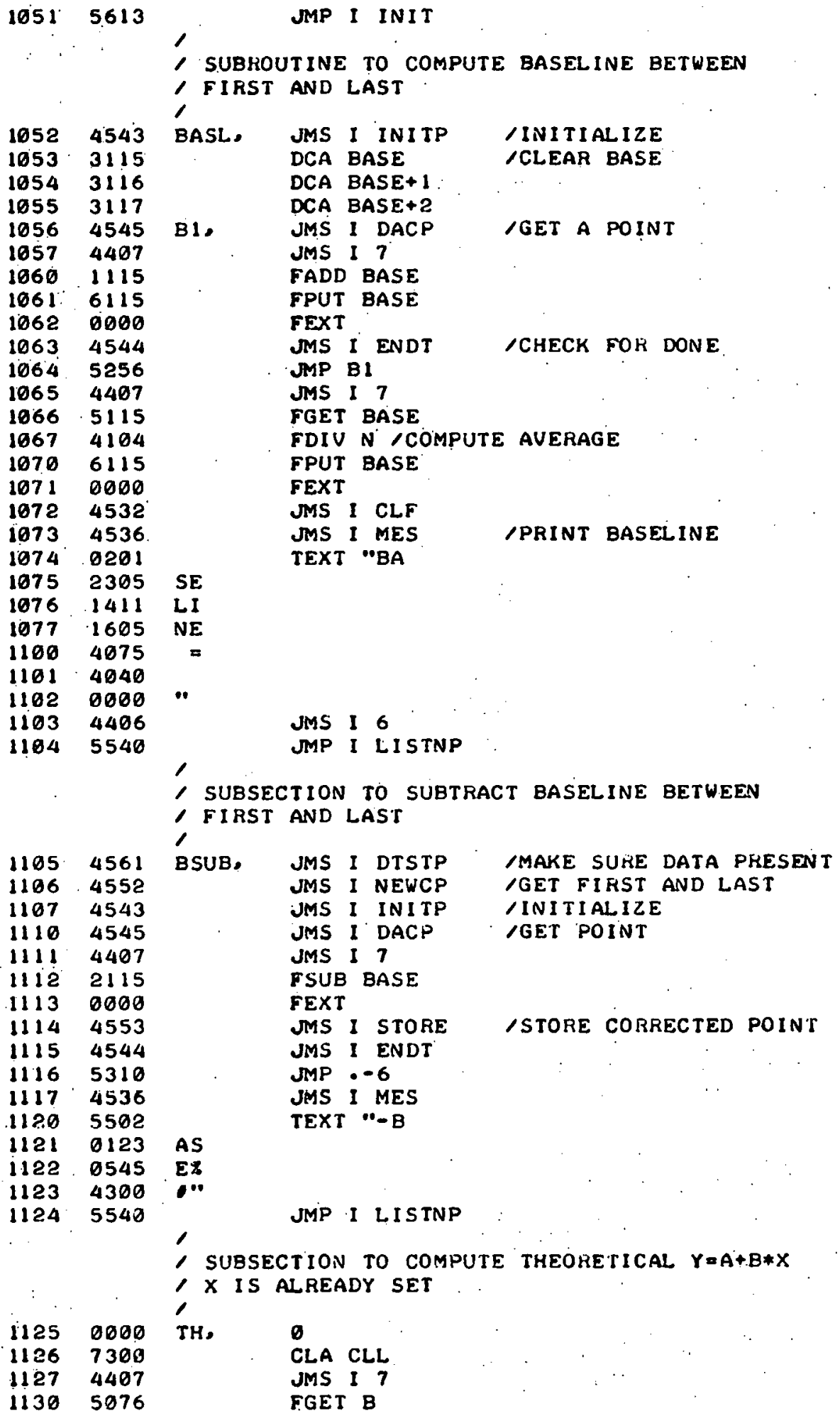




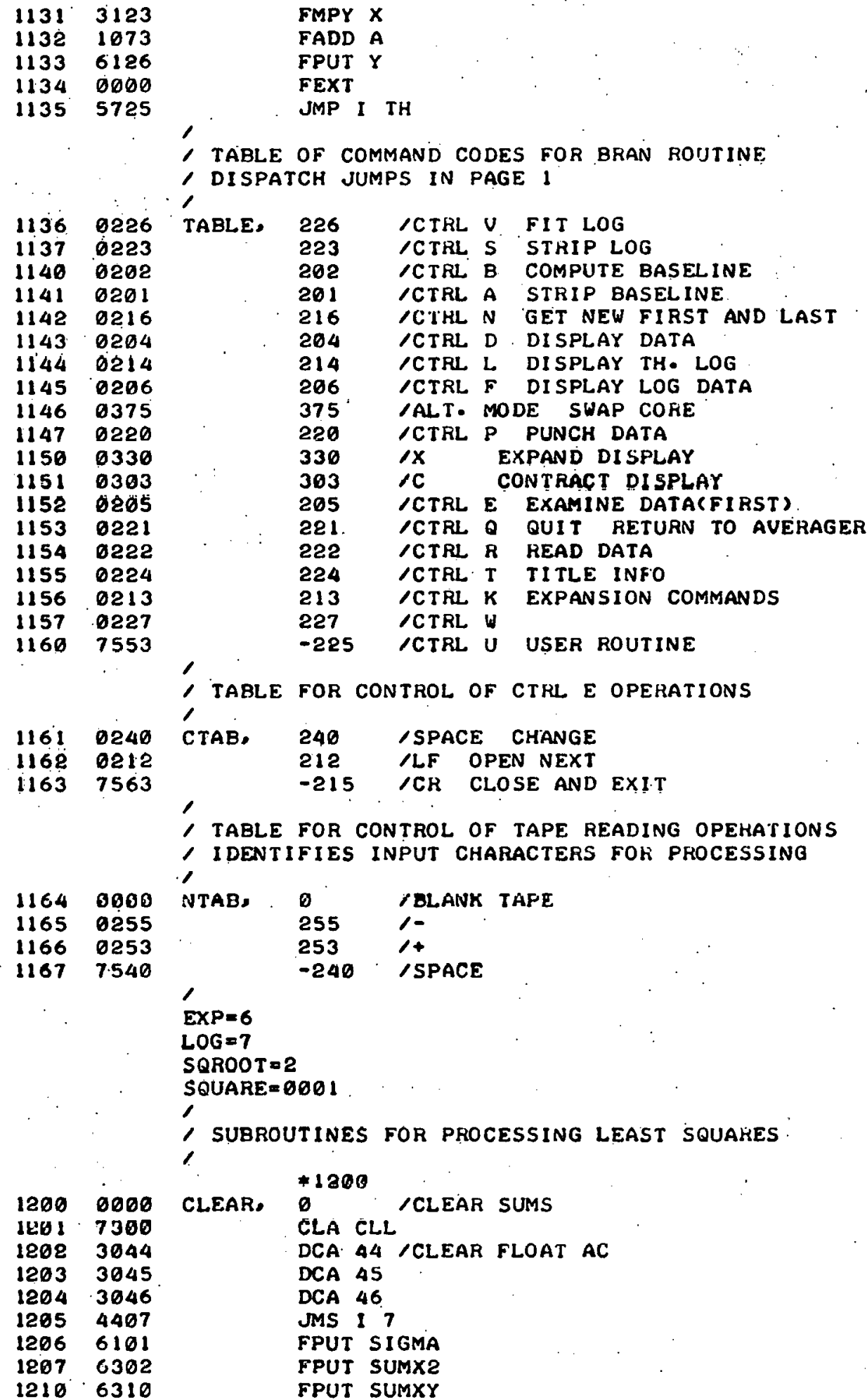




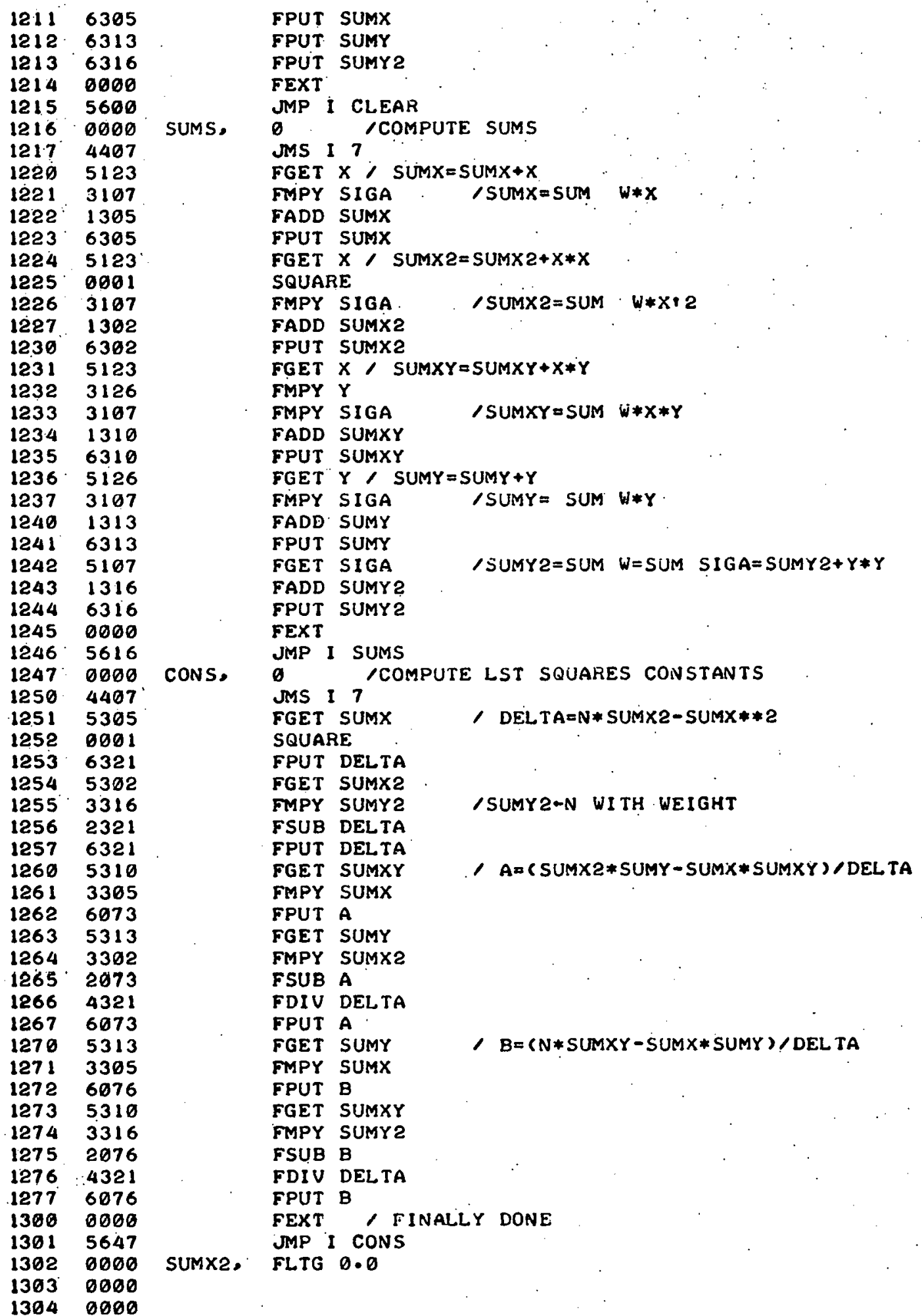




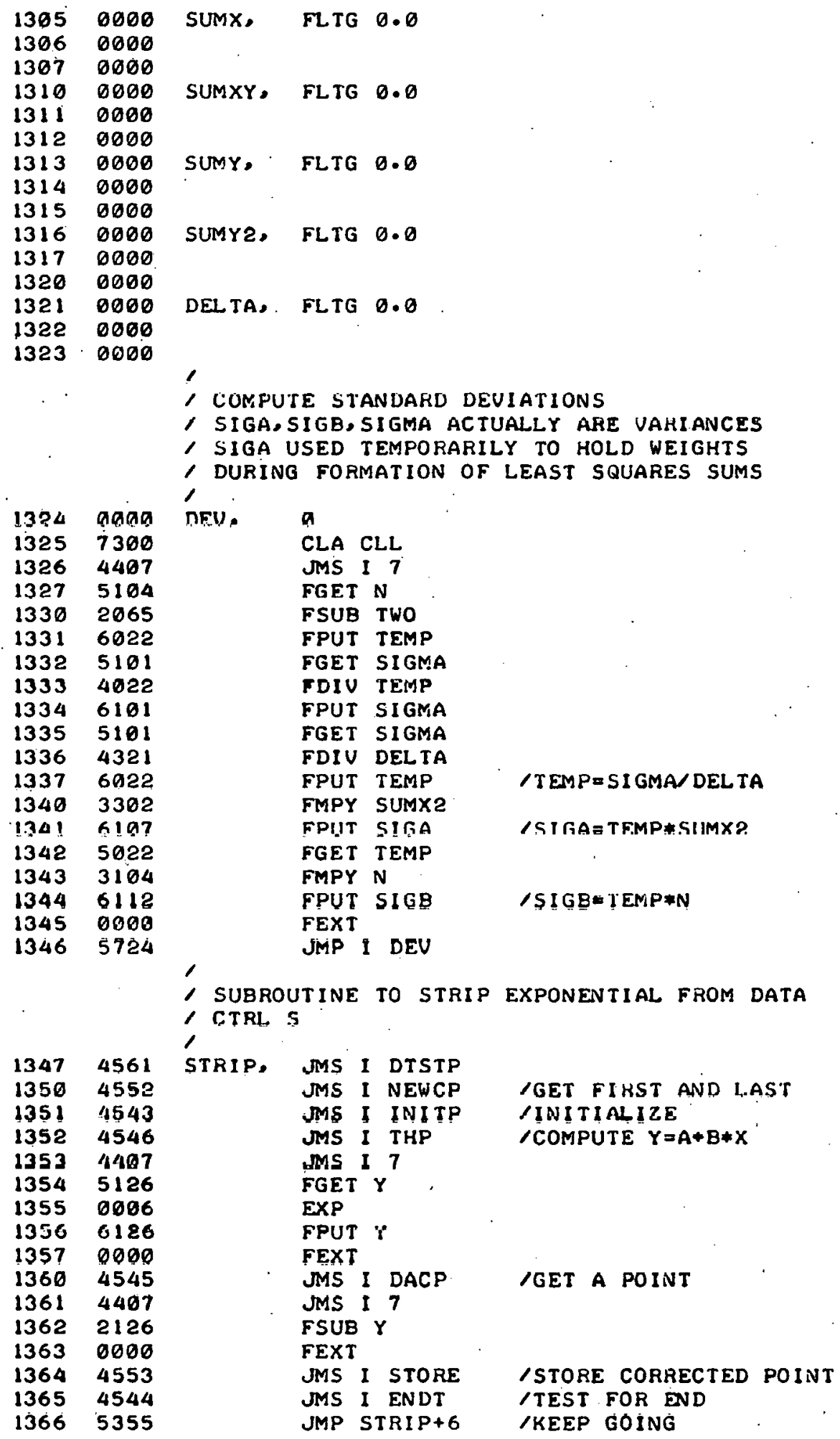




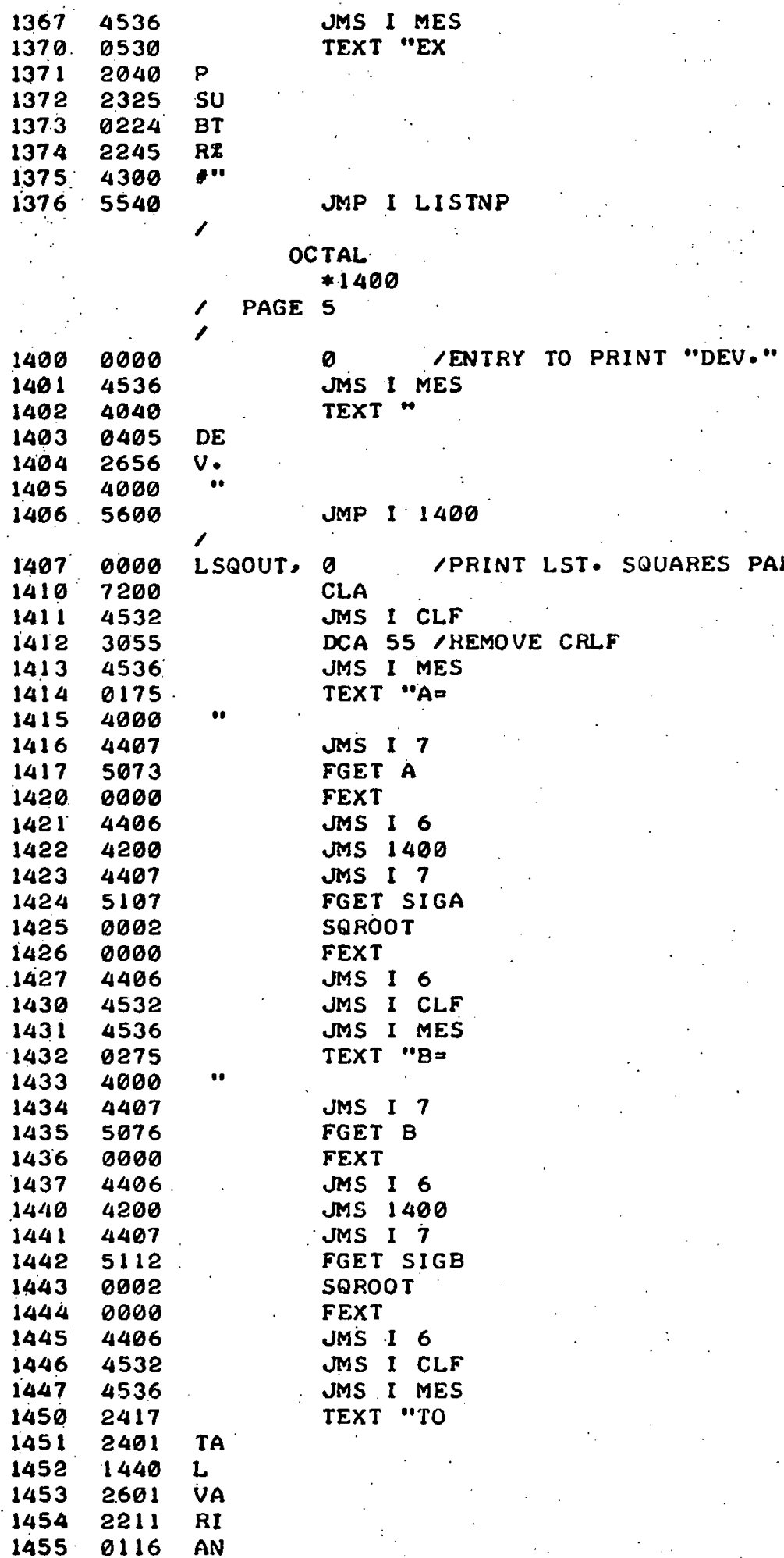




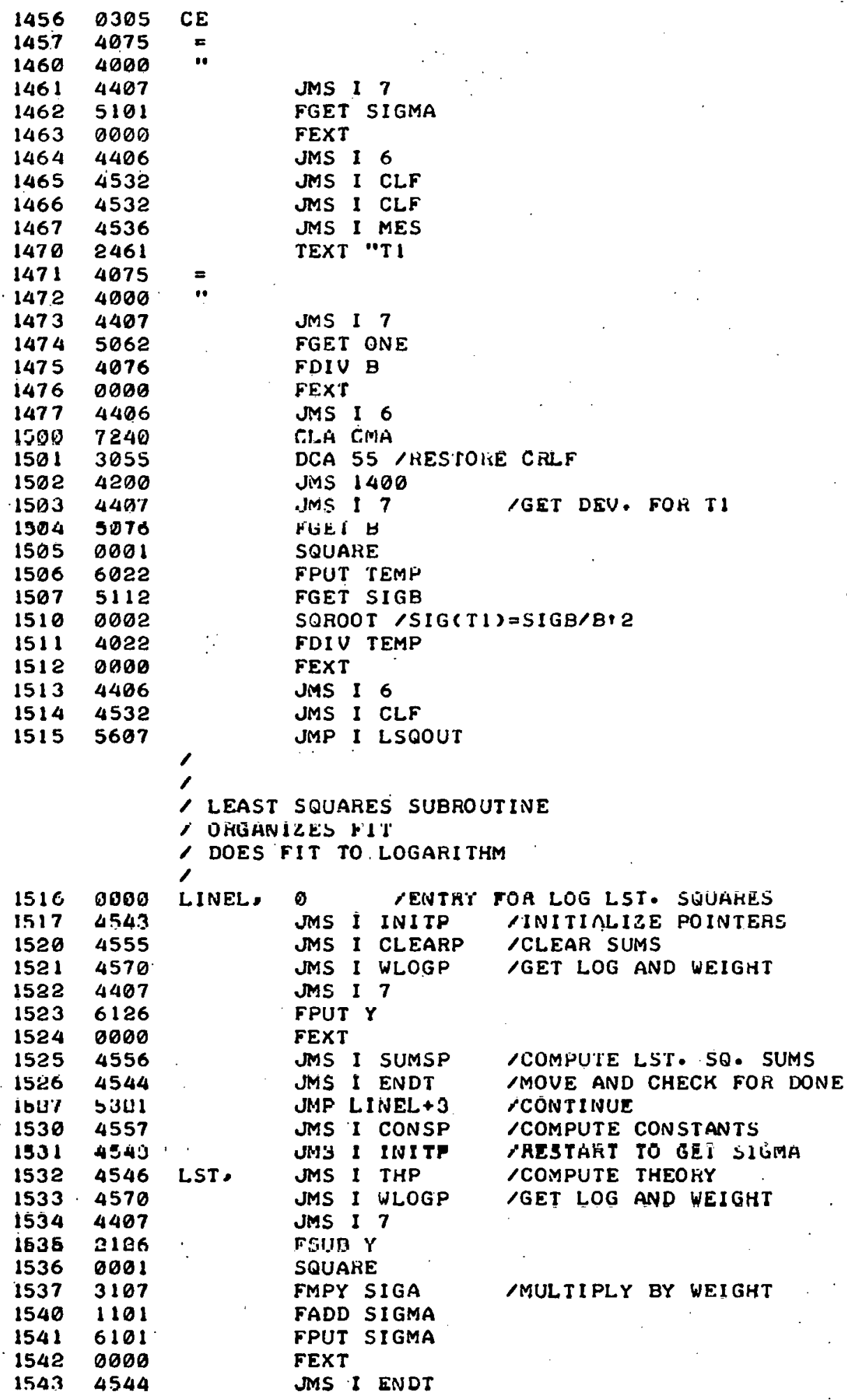




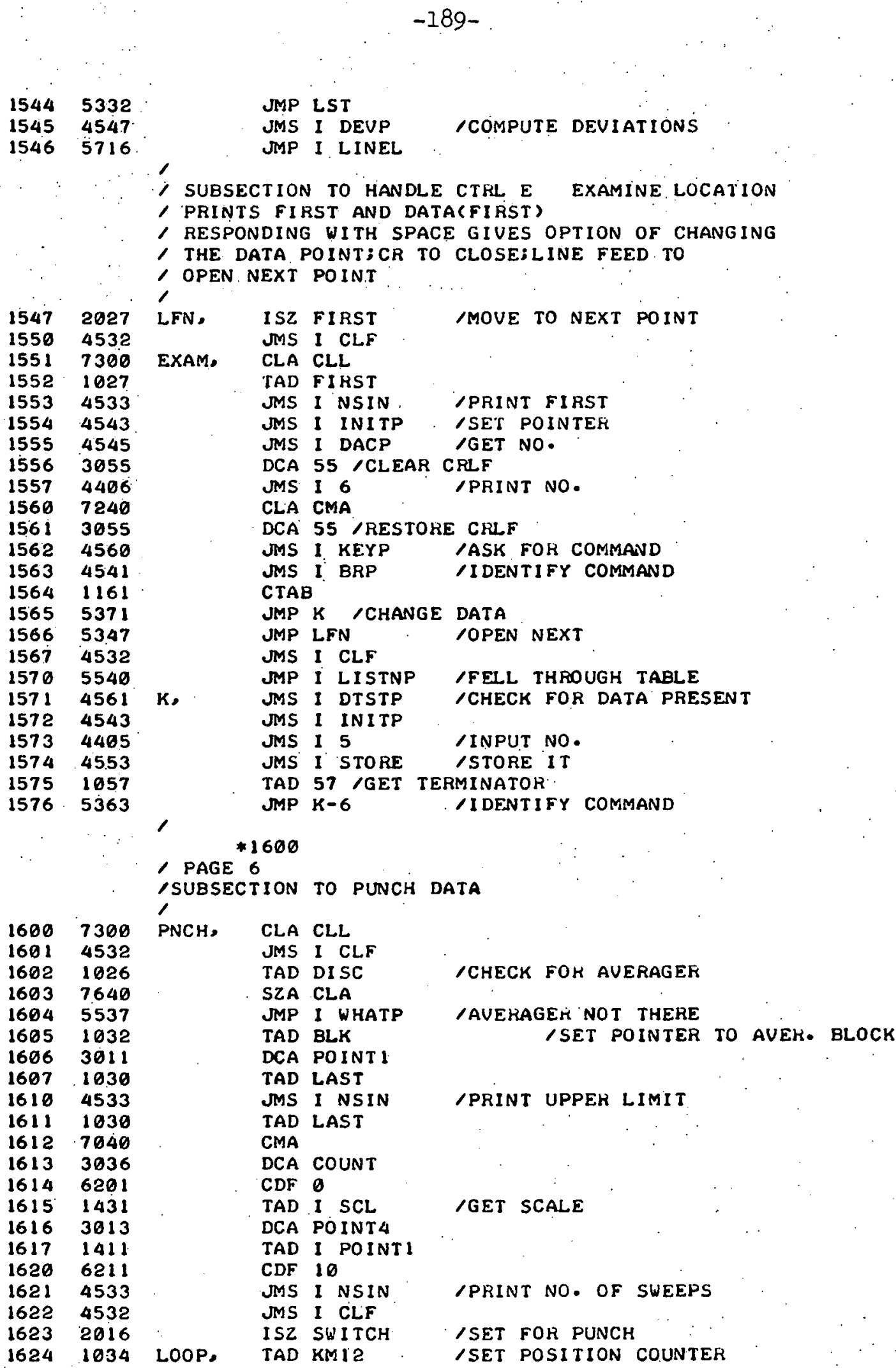




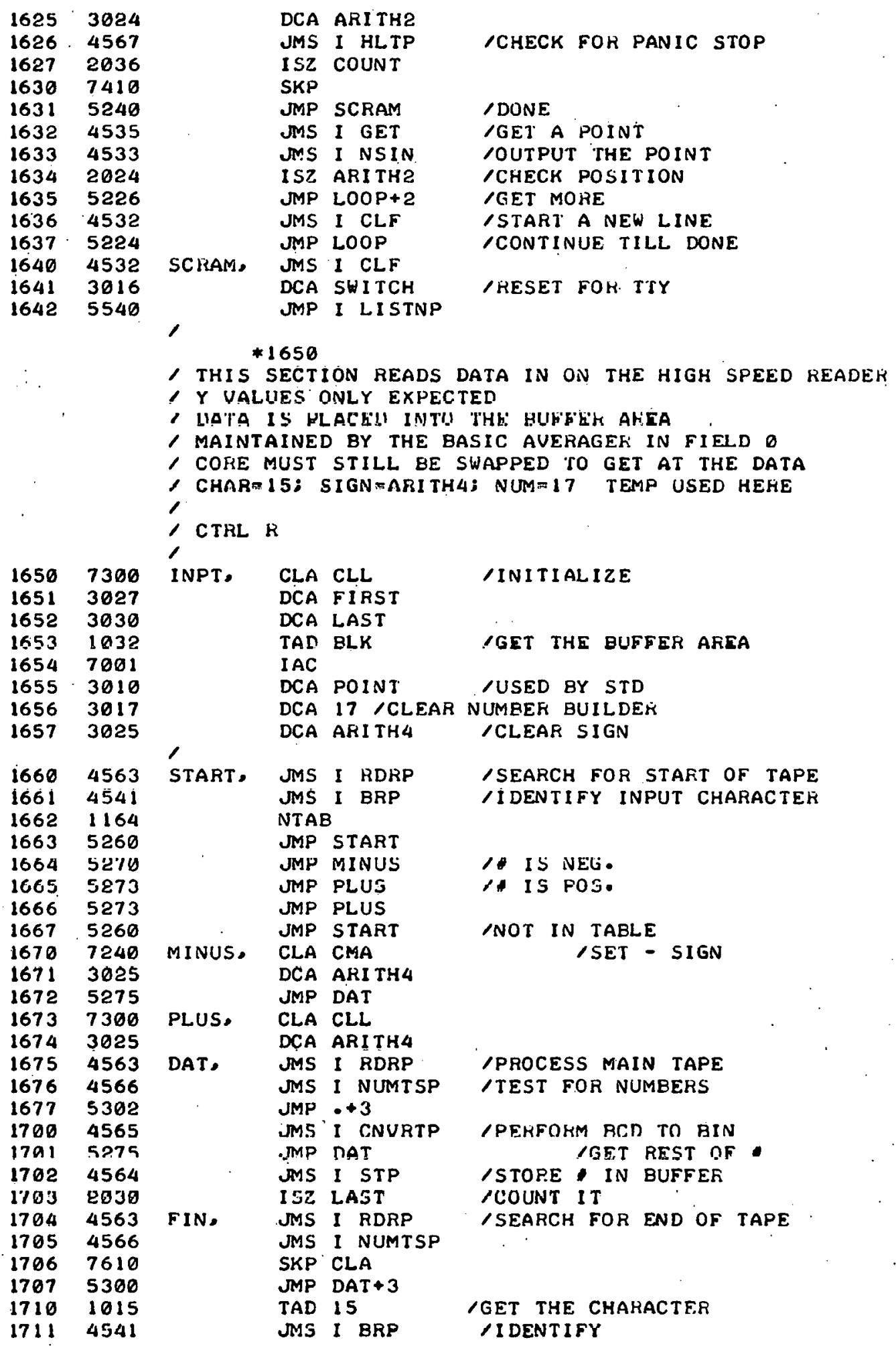




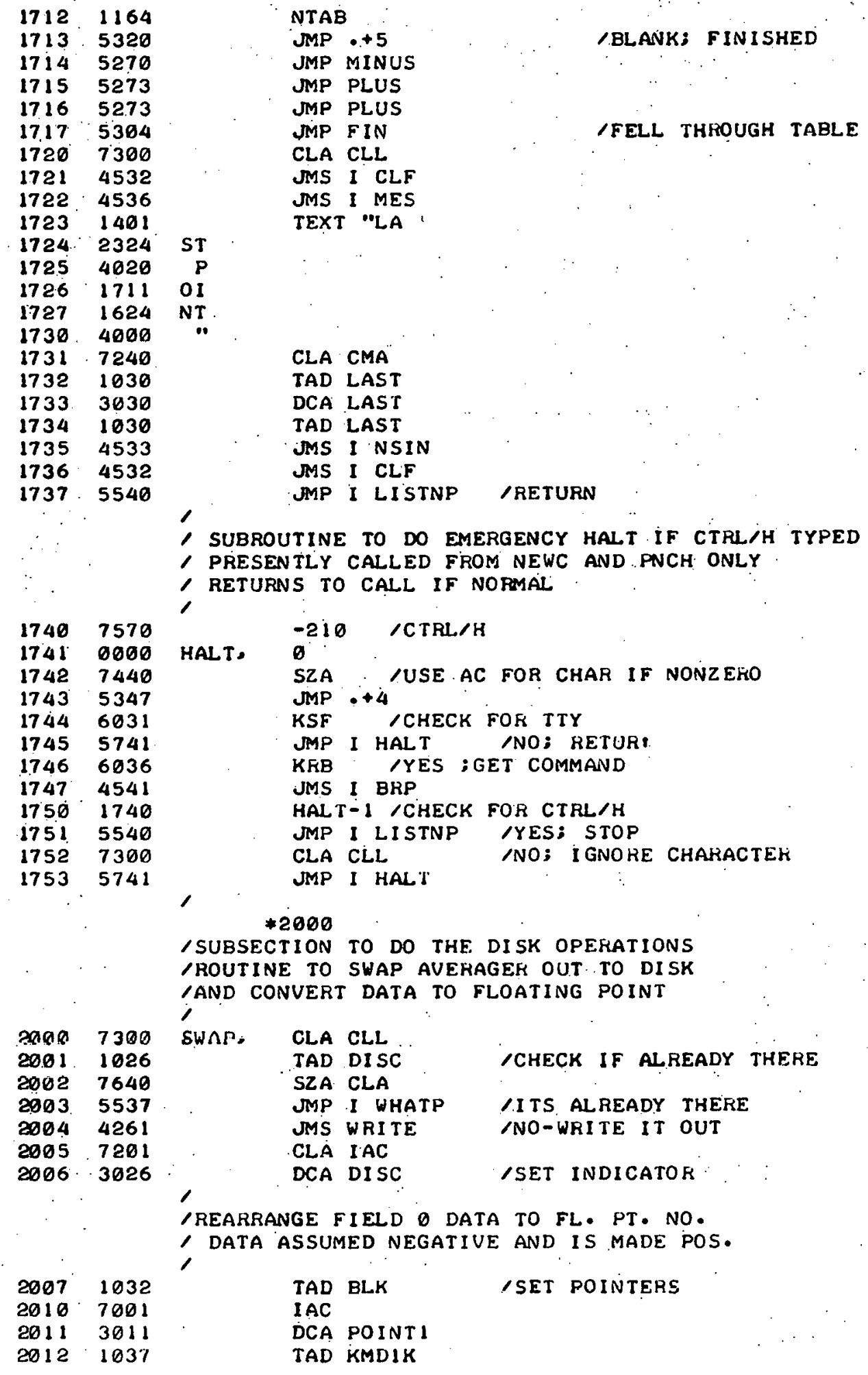




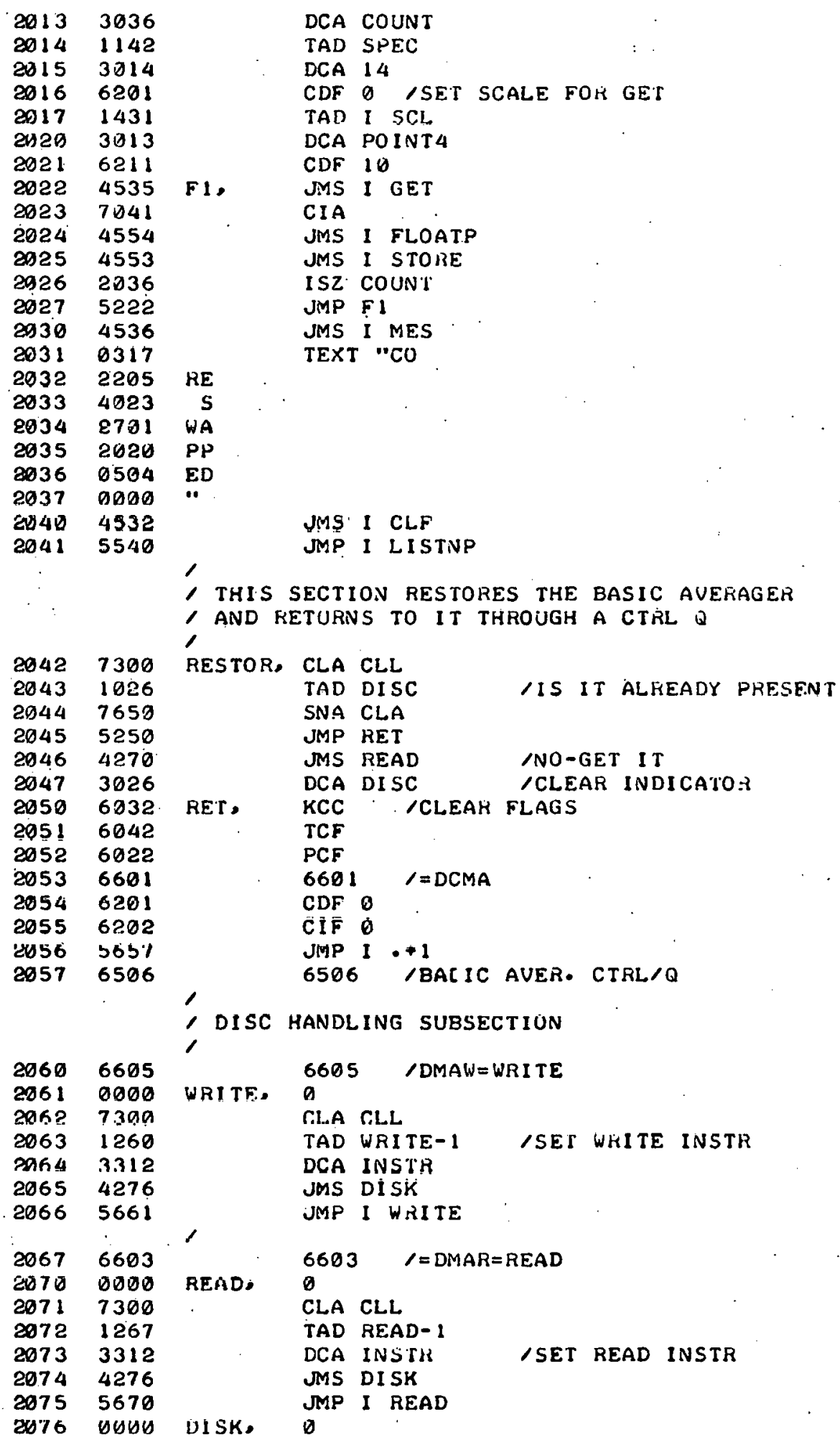




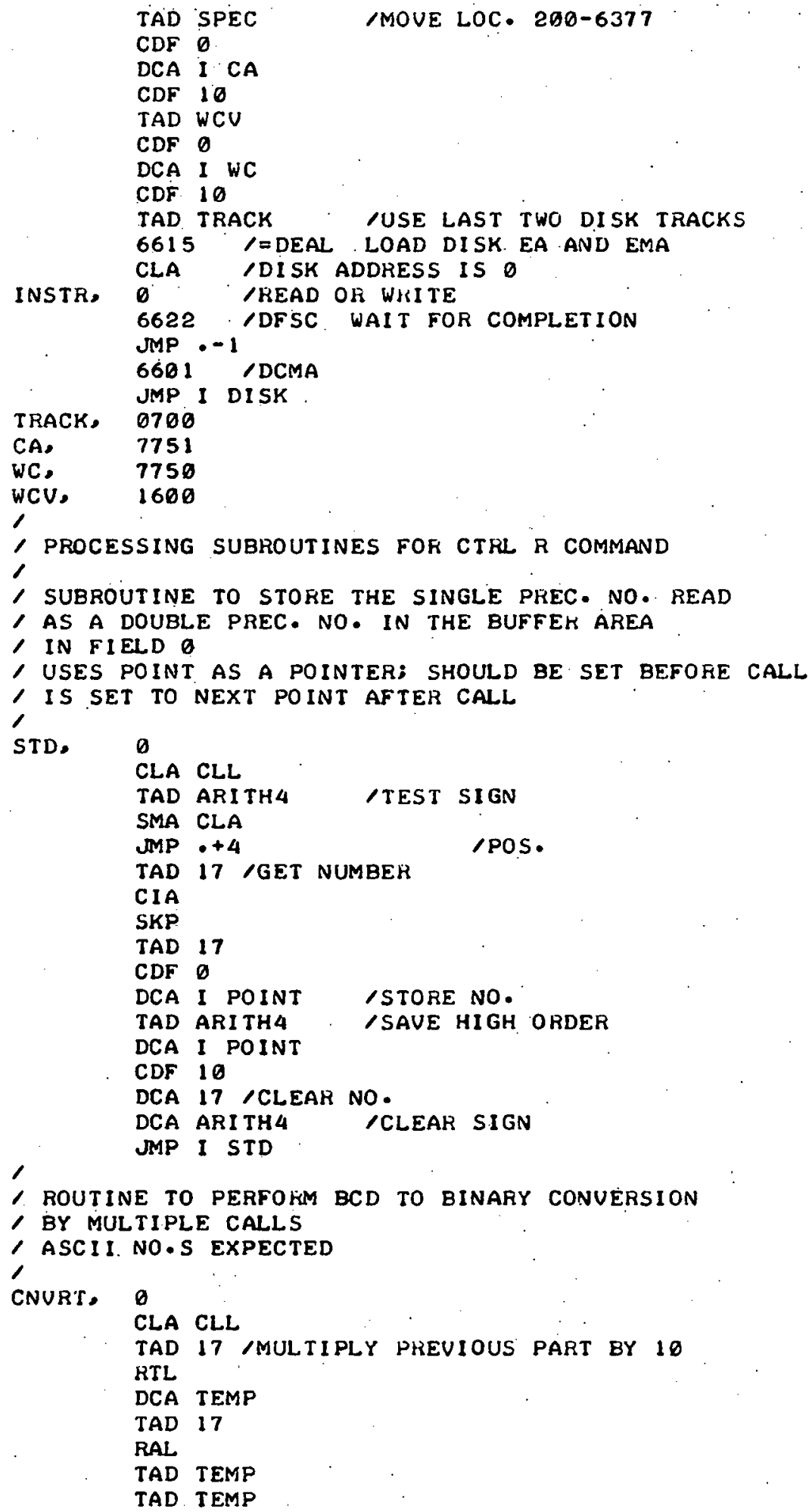




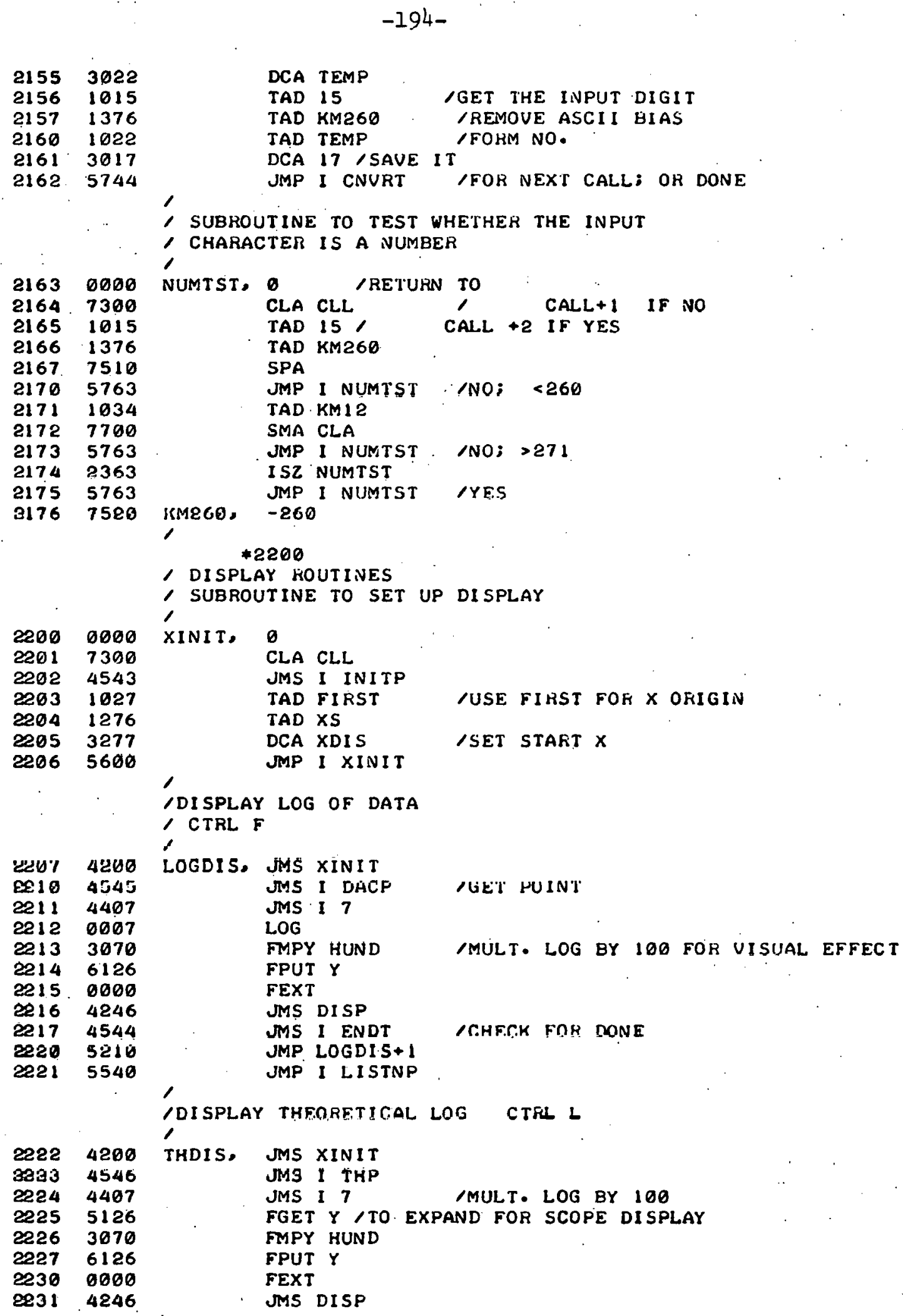

21553922

$2156 \quad 1015$

$2157 \quad 1376$

$2160 \quad 1022$

21613017

2162 5744

21630000

2164.7300

$2165 \quad 1615$

$2166 \quad 1376$

2167. 7510

$2170 \quad 5763$

$2171 \quad 1034$

$2172 \quad 7700$

$2173 \quad 5763$

21742363

$2175 \quad 5763$

3176 7580

$\begin{array}{ll}2200 & 0000 \\ 2201 & 7300 \\ 2202 & 4543 \\ 2203 & 1027 \\ 2204 & 1276 \\ 2205 & 3277 \\ 2206 & 5600\end{array}$

2सU 4260

EE10 4545

2211 4407

$2212 \quad 0007$

22133070

$2214 \quad 6126$

2215.0000

$2216 \quad 4246$

$2217 \quad 4544$

22205210

22215540

22224200

3893 4546

$2224 \quad 4407$

$2225 \quad 5126$

22263070

$2227 \quad 6126$

$2230 \quad 0000$

2831.4246 


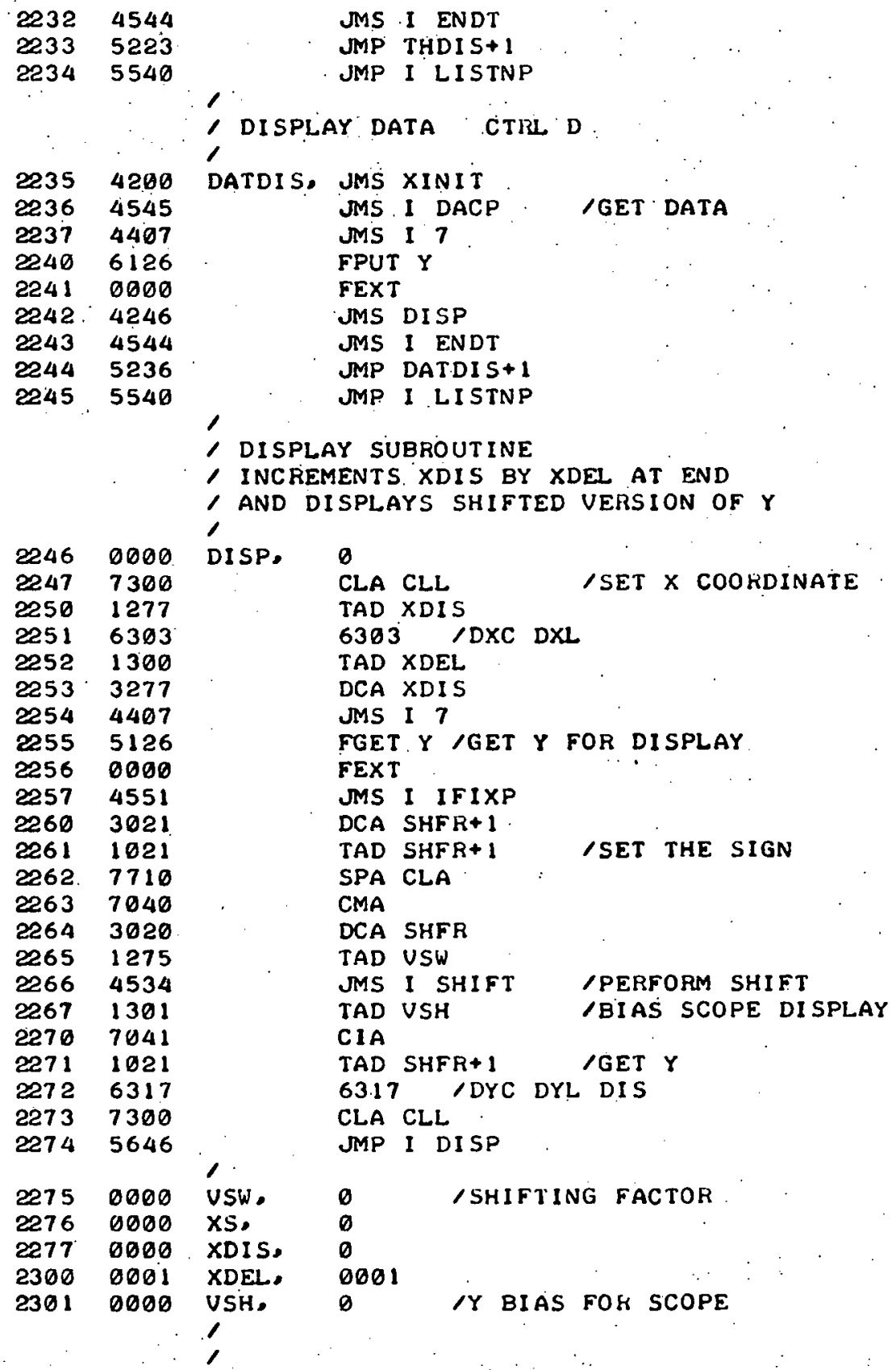


REFERENCES

A. Abragam, The Principles of Nuclear Magnetism(Oxford University Press, London, 1961).

A. Abragam and B. Bleaney, Electron Paramagnetic Resonance of Transition Ions (Oxford University Press, London, 1970).

S. A. Al'tshuler and K. A. Valiev, Zh. Eksp. Teor. Fiz. 35, 947(1958); Sov. Phys. JETP 10, 661(1959).

S. A. Al'tshuler and B. M. Kozyrev, Electrun Paramagnetlc Resundance (Academic Press, New York, 1964).

P. N. Argyres and P. L. Kelley, Phys. Rev. 134, A98(1964).

N. M. Atherton and G. R. Luckhurst, Mol. Phys. 13, 14.5(1967).

P. W. Atkins, Mol. Phys. 12, 133(1967).

P. W. Atkins and Daniel Kivelson, J. Chem. Phys. 44, 169(1966).

V. I. Avvakumov, Zh. Eksp. Teor. Fiz. 37, 1017(1959); Sov. Phys. JETP 10, $723(1960)$.

V. I. Avvakumov, N. S. Garif'yanov, B. M. Kozyrev, and P.'G. Tishkov, Zh. Eksp. Teor. Fiz. 37, 1564(1959); Sov. Phys: JETP 10, 1110(1960).

William Thomas Batchelder, The Electron Paramagnetic Resonance Spectra of $\alpha-\mathrm{NiSO}_{4}$ " $6 \mathrm{H}_{\mathrm{p}} \mathrm{O}$ at Liquid Helium Temperature(Ph. D. Thesis), UCRL-19157, May 1970.

Alfred Bauder and Rollie J. Myers, J. Mol. Spectrosc. 27, 110(1968).

George B. Benedek, Robert Englman, and John A. Armstrong, J. Chem. Phys: 39, 3349(1963).

I. B. Bersuker, Sov. Phys. JETP 17, 836(1963).

I. B. Bersuker and B. G. Vekhter, Sov. Phys. Sol. State I, 986(1965).

s. M. Blinder, J. Chem. Phys. 33, $148(1960)$.

F. Bloch, Phys. Rev. 70, 460(1946).

F. Bloch, Phys. Rev. 102, 104(1956).

F. Bloch, Phys. Rev. 105, 1206(1957).

N. Bloembergen, Phys. Rev. 104, 324(1956).

N. Bloembergen, E. M. Purcell, and R. V. Pound, Phys. Rev. 73, 679(1948). 
K. D. Bowers and W. B. Mims, Phys. Rev. 115, 285(1959).

D. P. Breen, D. C. Krupka, and F. I. B. Williams, Phys. Rev. 179, $241(1969)$.

J. Burgess and M. C. R. Symons, Quart. Rev. 22, 276(1968).

R. Calvo and R. Or:bach, Phys. Rev. 164, 284(1967).

A. Carrington and G. R. Luckhurst, Mol. Phys. \&, 125(1964).

Alan Carrington and Andrew D. McClachlan, Introduction to Magnetic Resonance (Harper \& Row, New York, 1967).

P. F. Cox and L. O. Morgan, J. Am Chem. Soc. 81, 64.09(1959).

C. F. Davis, Jr., M. W. P. Strandberg, and R. L. Kyhl, Phys. Rev. Ill, $1268(1958)$.

W. Edwards Deming, Statistical Adjustment of Data(John Wiley \& Sons, Inc., New York, 1938). Reprinted by Dover Publications, Inc., New York, 1964.

J. M. Deutch and Irwin Oppenheim, Adv. in Magnetic Resonance 3 , 43(1968).

Lawrence S. Frankel, J. Phys. Chem. 2, 736(1968).

George K. Fraenkel, J. Phys. Chem. 11, 139(1967).

Jack H. Freed, J. Chem. Phys. 49, 376(1968).

Jack H. Freed and George K. Fraenkel, J. Chem. Phys. 39, 326(1963).

Shizuo Fujiwara and Hisaharu Hayashi, J. Chem. Phys. 43, 23(1965).

Robert L. Fulton, J. Chem. Phys. 41, 2876(1964).

N. S. Garif'ianov and B. M. Kozyrev, Doklady Akad. Nauk S. S. S. R. 98 , 929(1954).

N. S. Garif'yanov, B. M. Kozyrev, R. Kh. Timerov, and N. F. Usacheva, Zh. Eksp. Teor. Fiz. 41, 1076(1961); Sov. Phys. JETP 14, 7.68(1962a).

N. S. Garif'yanov, B. M. Kozyrev, R. Kh. Timerov, and N. F. Usacheva, Zh. Eksp. Teor. Fiz. 42, 1145(1962); Sov. Phys. JETP 15, 791(1962b).

N. S. Garif'yanov and N. F. Usacheva, Russ. J. Phys. Chem. 38, 752(1964).

H. R. Gersmann and J. J. S'walen, J. Chem. Phys. 36́, 3'2'L1(196́2).

L. van Gerven, J. Talpe, and A. van Itterbeck, Physica 33, 207(1967). 
J. C. Gill, Proc. Phys. Soc. (Lond.) 85, 119(1965).

C. J. Gorter, Paramagnetic Relaxation(Elsevier Publishing Co., Amsterdam, 1947).

Robert G. Hayes, Electron Spin Resonance Line Widths of Transition Metal Ions and Complexes in Solution(Ph. D. Thesis), UCRL-9873, sept. 1961.

L. C. Hebel and C. P. Slichter, Phys. Rev. 113, 1504(1959).

Paul S. Hubbard, Phys. Rev. 131, 1155(1963).

A. Hudson and G. R. Luckhurst, Chem. Revs. 69, 191(1969).

James A. Ibers and J. D. Swalen, Phys. Rev. 127, 1914(1962).

H. A. Jahn, Proc. Roy. Soc. (Lond.) 164, 117(1938).

H. A. Jahn and E. Teller, Proc. Roy. Soc. (Lond.) 161, 220(1937).

Akira Jindo, Single Crystal Studies of Hydrated Transition Metal Ions by Electron Paramagnetic Resonance(Ph. D. Thesis), UCRL-20386, 1971.

Theron S. Johnston and Harry G. Hecht, J. Mol. Spectrosc. 17, 98(1965).

J. B. Jones and M. F. Lewis, Solid State Commun. 5, 595(1967).

Wilfred Kaplan, Ordinary Differential Equations(Addison-Wesley Publishing Company, Inc., Reading, Mass., 1958).

Daniel Kivelson, J. Chem. Phys. 27, 1087(1957).

Daniel Kivelson, J. Chem. Phys. 33, 1094(1960).

Daniel Kivelson, J. Chem. Phys. 4.1, 1904 (1964).

Daniel Kivelson, J. Chem. Phys. 45, 751(1)(1966a).

Daniel Kivelson, J. Chem. Phys. 4.5, 1324(1966b).

Daniel Kivelson and George Collins, ESR Line Widths in Liquids, in Paramagnetic Resonance, Vol. II, W. Low, Ed.(Academic Press, New York, 1963), p. 496.

Daniel Kivelson and Robert Neiman, J. Chem. Phys. 35, 149(1961).

Fritz Kurt Kneubuhl, J. Chem. Phys. 33, 1074(1960).

B. M. Kozyrev, Disc. Faraday Soc. 19, 135(1.955). 
B. M. Kozyrev, Izv. Akad. Nauk S.S.S.R. ser.Fiz. 2l, 828(1957)(Eng. Trans). Ryogo Kubo and Katushị Tomita, J. Phys. Soc. Japan 9, 888(1954).

Ronald Lee, James Neely, and Rochelle (Cooper) Dreyfuss, privàte communication(1968).

W. Burton Lewis, Mohammed Alei, Jr., and L. O. Morgan, J. Chem. Phys. 4.4, 24.09(1966).

W. Burton Lewis and L. O. Morgan, Paramagnetic Relaxation in Solutions, in Transition Metal Chemistry, Vol. 4, R. Carlin, Ed. (Marcel Dekker, Inc., New York, 1968).

M. F. Lewis and A. M. Stoneham, Phys. Rev. 164, 271(1967).

A. H. Maki and B. R. McGarvey, J. Chem. Phys. 29, 31(1958a).

A. H. Maki and B. R. McGarvey, J. Chem. Phys. 29, 35(1958b).

Douglas C. McCain, The Measurement of Eilectron Spin Relaxation Times of Ions in Aqueous Solution(Ph. D. Thesis), UCRL-17064, Aug. 1966.

Douglas C. McCain and Roilie J. Myers, J. Phys. Chem. 71, 192(1967).

A. D. McClachlan, Proc. Roy. Soc. (Lond.) 280, 271(1964).

R. E. D. MCClung and Daniel Kivelson, J. Chem. Phys. 49, 3380(1968).

Harden M. McConnell, J. Chem. Phys. 25, 709(1956).

B. R. McGarvey, J. Phys. Chem. 60, 71(1956).

B. R. McGarvey, J. Phys. Chem. 61, 1232 (1957).

Charles W. Merideth, Temperature Dependence of Transverse Relaxation Times of Oxygen-17 in Aqueous Solutions Containing Cupric and Chromous Ions(Ph. D. Thesis), UCRL-11704, Jan. 1965.

W. B. Mims, K. Nassau, and J. D. McGee, Phys. Rev. 123, 2059(1961).

L. O. Morgan and A. W. Nolle, J. Chem. Phys. 31, 365(1959).

Rober.t Neiman and Daniel Kivelson, J. Chem. Phys. 35, 156(1961).

Mary C. M. O'Brien, Proc. Roy. Soc. (Lond.) A281, 323(1964).

Lavid Wixon Fratt, Magnetic Resonance spectra of $\mathrm{VCI}_{4}$ and other

Paramagnetic Species(Ph. D. Thesis), UCRL-17406, April 1967. 
U. Op1k and M. H. L. Pryce, Proc. Roy. Soc.(Jond.) 123 ?, 425(195\%).

G. E. Pake and R. H. Sands, Phys. Rev. 98, 266(1)(1955).

A. G. Redfield, IBM J. Res. Dev. 1, 19(1957).

A. G. Rodf eld, The Theory of Relaxation Processes, in Adv. in Magnctic

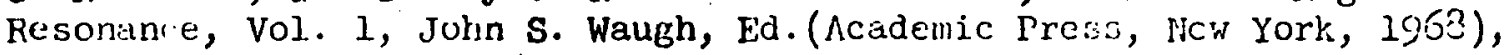
p. 1 .

J. Reuben ind D. Flat, Inorg. Chem. 6, 579(1967).

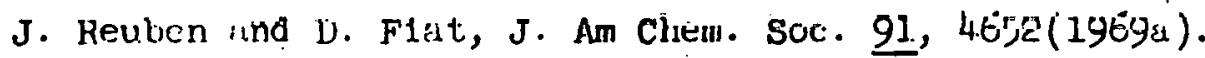

J. Reuben and D. Fiat, Inorg. Chem. \&, 1821(1969b).

R. N. Rogers and G. E. Pake, J. Chem. Phys. 33, 1107(1960).

Robert T. Jioss, J. Chem. Phys. 42, 3919(1965).

D. Sames, :'ieit. fur Physik 188, 108(1965).

D. Sames, Zeit. fur Physik 198, 71(196'7).

R. H. Sands, Phys. Rev. 99, 1222(1955).

Abraham Savitzky and Marcel J. E. Golay; Anal. Chem. 36, 1627(1964). Jan W. Schreurs and Daniel Kivelson, J. Chem. Phys. 36, 117(1962).

P. L. Scott and C. D. Jeffries, Phys. Rev. 127, 32(1962).

Joel Selbin, Chem. Rev. 65, 153(1965).

Joel Selbin and L. H. Holmes, Jr., J. Inorg. Nucl. Chem. 24, 1111(1962).

David P. Shoemaker and Carll W. Garland, Experiments in Physical Chemistry (McGraw-Hill Book Company, Inc., New York, 1962).

Hans Sillescu and Daniel Kivelson, J. Chem. Phys. 48, 3493(1968).

E. Simanek and R. Orbach, Phys. Rev. 145, 191(1966).

Charles P. Slichter, Principles of Magnetic Resonance(Harper \& Row, New York, 1963).

F. J. Simith, J. K. Smith, and S. P. McGlynn, Rev. Sci. Instrum. 33, $1367(1962)$.

Zoltan G. Soos, J. Chem. Phys. 49, 2493(1968). 
John B Spencer, Electron Spin Resonance Studies of Trar sition Hetal. Jon Complexes(Ph. D. Thesis), UCRL-16175, Aug. 1965.

K. J. Standley and R. A. Vaughan, Electron spin Ralaxation Phenoment in Solids(Plenum Press, New York, 1969).

K. W. H. Stevens, Rept. Prog. Physies XXX part.1, 129(196\%).

A. M. Stonchain, Proc. Phys. Soc. (I,ond.) 85, 107(1965).

J. D. Swalen and H. M. Gladney, IIM J. Res. Dev. 8, 515(.2964).

T. J. Swift and Robert E. Connick, J. Chem. Phys. 37, 307( J.96e ).

E. E. Vainshtein and I. I. Antipova-Karataeva, Russ. J. Inorg. Chem. 4, 355 (1959).

K. A. Valiev and M. M. Zaripov, 2h. Eksp. Teor. Fiz. 4l, 756(1961); Sov. Phys. JETP 14, 545(1962).

K. A. Valiev and M. M. Zaripov, Opt. Spektrosk. 20, 108(1966); Optics \& Spect. 20, 56(1966).

R. M. Valishev, Sov. Phys. Sol. St. I, 733(1965).

Tore Vanngard and Roland Aasa, ESR Line Shapes of Polycrystalline Samples of $S^{\prime}=1 / 2$ Transition Element Ions, in Paramagnetic Resonance Vol. II, W. Lọw, Ed. (Academic Press, New York, 1963), p. 509.

J. H. van Vleck, J. Chem. Phys. I, 72(1939).

G. P. V1shnevskaya and B. M. Kozyrev, Zh. Strukt. Khimi1 6, 667(1965); J. Struct. Chem. 6, 637(1965).

Walter M. Walsh, Jr., Jean Jeener, and N. Bloembergen, Phys. Rev. 139A, $1338(1965)$.

R. K. Wangsness and F: Bloch, Phys. Rev. 89, 728(1953).

F. I. B. Willians, D. C. Krupka, and D. P. Breen, Phys. Rev. 179, 255 (1969).

Raymond Wilson and Daniel Kivelson, J. Chem. Phys. 44, 154.(1966a).

Raymond Wilson and Daniel Kivelson, J. Chem. Phys. 44, 4.440(1966b).

Raymond Wilson and Daniel Kivelson, J. Chem. Phys. 44, 44.45(1966c).

Amnon Yariv and W. H. Louisell, Phys. Rev. 125, 558(1962). 
Allan Zalkin, J. D. Forrester, and David H. Templeton, J. Chsm. Phys. 32, $2881(1963)$.

M. M. Zaripov, opt1c8 \& spect. 18, 136(1965). 


\section{ACKNOWJ FIDGMENTS}

Many people have helped to make my stay in Berkeley a most enjoyable experience. I would like to thank my reseurch director, Professor Rollie J. Myers, for his patience and cuidance during my many years of graduate school. IIis expertise in getting experimental set-ups to work has been very bencficial; it has been educational to watch him work. The members of my research group have been most helpful. Tre conversations which I have had with Doug McCain, Dave Pratt, and Aki Jindo have been very stimulating and in many cases guided my thoughts along lines which I may not otherwise have followed. I would also like to acknowledge Fredi Bauder who wrote the inftial versions of the data acquisition system programs; also Raymond Pao, Tom Hynes, and Joyce Tevebaugh; and, of course, Eloise.

I would like to thank the many friends which I have made in Berkeley for their comradeship and encouragement, especially my roommates. My asscciation. with the 5th floor, particularly with the members of the Jolly group, has enriched my life. The many "quickies" were enjoyable and a welcome break from sometimes tedious research.

This work was performed under the auspices of the United States Atomic Energy Commission through the Inorganic Materials Research Division of the Lawrence Radiation Laboratory. 
This report was prepared as an account of work sponsored by the United States Government. Neither the United States nor the United States Atomic Energy Commission, nor any of their employees, nor any of their contractors, subcontractors, or their employees, makes any warranty, express or implied, or assumes any legal liability or responsibility for the accuracy, completeness or usefulness of any information, apparatus, product or process disclosed, or represents that its use would not infringe privately owned rights. 
